\title{
Periodontal Health AND HIV InFeCtion
}

A thesis submitted to the University of London in fulfilment for the degree of Doctor of Philosophy in the Faculty of Medicine (Dentistry).

\section{Peter Glenn Robinson}

Department of Epidemiology and Public Health, University College London, 1-19 Torrington Place, London WC1E 6BT.

Department of Oral Medicine and Pathology, United Medical And Dental Schools of Guy's and St Thomas' Hospitals, Floor 28, Guy's Tower, Guy's Hospital, London Bridge, London SE1 9RT 
ProQuest Number: 10044405

All rights reserved

INFORMATION TO ALL USERS

The quality of this reproduction is dependent upon the quality of the copy submitted.

In the unlikely event that the author did not send a complete manuscript and there are missing pages, these will be noted. Also, if material had to be removed, a note will indicate the deletion.

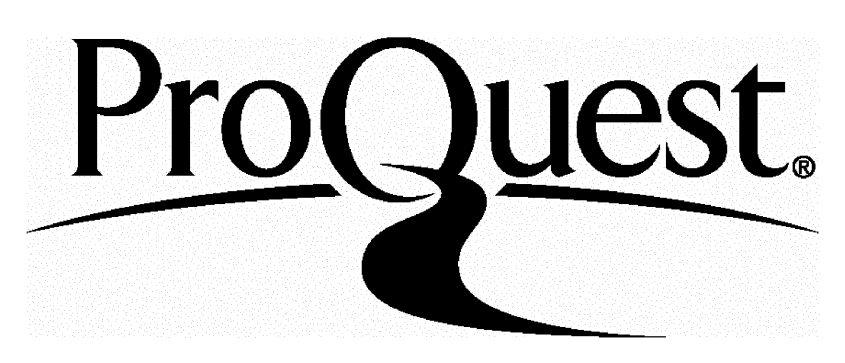

ProQuest 10044405

Published by ProQuest LLC(2016). Copyright of the Dissertation is held by the Author.

All rights reserved.

This work is protected against unauthorized copying under Title 17, United States Code. Microform Edition (c) ProQuest LLC.

\author{
ProQuest LLC \\ 789 East Eisenhower Parkway \\ P.O. Box 1346 \\ Ann Arbor, MI 48106-1346
}




\section{$\underline{\text { Abstract }}$}

There has been debate about whether people with HIV experience unusual or severe periodontal diseases. The research carried out for this thesis comprises 4 epidemiological studies of periodontal health and HIV infection.

The first study aimed to develop diagnostic criteria for periodontal changes associated with HIV. Criteria were derived by experienced dentists and tested in a formal experiment. Training with the criteria improved diagnostic reliability.

The second study aimed to identify the types, prevalence and severity of periodontal changes seen in HIV infection in a controlled blinded study of 794 homosexual men attending a genito-urinary medicine clinic. Regression analyses associated HIV infection with periodontal pockets, attachment loss, conventional gingivitis, gingival ulceration and erythema of the attached gingiva. HIV disease progression predicted attachment loss but not pocketing. Gingivitis and erythema of the attached gingiva were associated with oral candidiasis.

The third study aimed to describe gingival ulceration seen in HIV infection and identify possible aetiological factors. 95 cases were reviewed and compared against 269 controls with HIV. The most common presentation was clinically and microbiologically similar to acute necrotising ulcerative gingivitis. Incidence of this disease was inversely related to age and health and associated with oral candidiasis.

Finally, to validate diagnostic criteria for HIV-associated periodontal changes, their predictive values for HIV infection were assessed by applying them post-hoc to data from the cross-sectional study. Criteria sensitive to erythema of the attached gingiva and interdental attachment loss had high predictive values. Three HIV-associated periodontal changes were recognised: erythema of the attached gingiva; necrotising periodontal disease and reverse architecture/interdental craters.

A number of periodontal changes are associated with HIV infection. Those specifically associated with the infection are less common than originally suggested. Diagnostic criteria sensitive to the specific and non-specific changes are proposed. 


\section{Acknowledgements}

The custom of acknowledging those who contribute to a body of work diminishes the gesture. People may be thanked merely because it is customary. In this case there are a number of people who must be acknowledged sincerely, not only for lending support, but also for making the work such a positive experience and for making it fun.

Professors Aubrey Sheiham and Stephen Challacombe have supervised this research and have been unstintingly generous with their experience and knowledge. It has been a privilege to work with them both and I hope to continue learning from them in future.

Dr. Joanna Zakrzewska provided early impetus and support. Other staff at that clinic have assisted by their vigilant examination of patients, careful record keeping and observance of investigation and treatment protocols, particularly Heiko Cooper, Ian Greenwood and Jane Hatt.

Guy Palmer (UMDS) and Jim Winkler (UCSF) worked on the development of diagnostic criteria and members of the London HIV and Dentistry Group helped to hone the criteria in some lively debate. Drs. Gary Armitage, Michael Danford, Caroline Dodd, Francine Lozada-Nur, Gad Heinic, Shepherd Levine, Laurie MacPhail and David Richards of the Division of Stomatology at the University of California at San Francisco took part in the calibration exercises. I am especially grateful to them for submitting their clinical skills to critical analysis. Drs. Deborah and John Greenspan and Mitch Katz of UCSF all helped to refine the work for that project and Janice Westenhouse and Joan Hilton assisted with the data analysis.

The cross-sectional study would not have been possible without the staff of James Pringle House (now part of The Mortimer Market Centre) who recruited participants, located clinical information and tolerated the burden we placed on their clinics for 15 months. Laurie Wilkinson was the research assistant throughout this period. The success of this project was wholly dependent on her diligence, enthusiasm and considerable skills.

Mr. Michael Wren, Senior MLSO at University College Hospital carried out all the microbiological investigation of the samples from patients with gingival ulceration. Dr. Wagner Marcenes of the Department of Epidemiology and Public Health at UCL became an unwitting but able statistics tutor. Dr. Trevor Watts of the Department of Periodontology at 
UMDS shared his knowledge of all aspects of periodontology, particularly clinical research methodology with kindness and enthusiasm.

Julie Weeks and our children have made considerable sacrifices to allow me to complete this work. It would not have been the same without them.

I must express my affection for my patients at the Mortimer Market Dental Clinic and my thanks to those at the James Pringle House. They have taught me much over the last 13 years. They have taught me about disease, about illness and about death. More importantly they have taught me about life, about dignity and about power.

Financial support for this work was provided by a Smithkline Beecham Research Fellowship of the Oral and Dental Research Trust. The reliability studies for diagnostic criteria were funded in part from a Colgate Research Award, also via the Oral and Dental Research Trust and through the National Institute for Dental Research program project grant P01-DE07946. I am grateful for the confidence placed in my by these bodies, particularly Professors Declan Anderson and Tony Naylor of the Oral and Dental Research Trust.

Smithkline Beecham kindly provided $1 \frac{1}{2}$ tons of personal hygiene products to be used as inducements in the cross-sectional study which greatly aided recruitment. Drs. Geoff Forward and Mike Shaw have taken an interest in the work throughout and I wish to thank them for their support too. 


\section{TABLE OF CONTENTS}

$\begin{array}{lllllllllll}\text { Title page } & - & - & - & - & - & - & - & - & & 1\end{array}$

Abstract $\quad 2$

Acknowledgements $\quad 3$

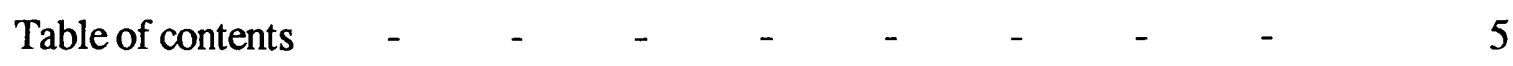

$\begin{array}{ll}\text { List of tables } & 10\end{array}$

$\begin{array}{ll}\text { List of figures } & 12\end{array}$

CHAPTER 1 INTRODUCTION AND OVERVIEW OF THE THESIS 14

CHAPTER 2 BACKGROUND AND REVIEW OF THE LITERATURE

$\begin{array}{lll}2.1 & \text { HIV and AIDS } & 15\end{array}$

2.1.1 Epidemiology 15

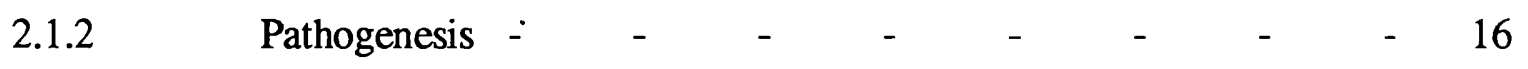

2.1.3 Clinical presentation. 17

2.1.3.1 Oral Manifestations of HIV infection 18

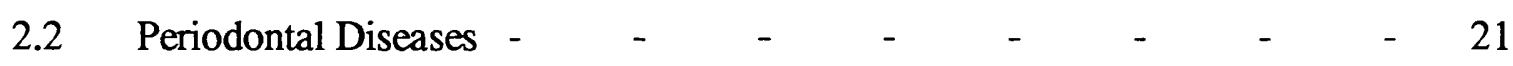

$\begin{array}{lll}2.2 .1 & \text { Gingivitis } & 21\end{array}$

2.2.2 Periodontitis 23

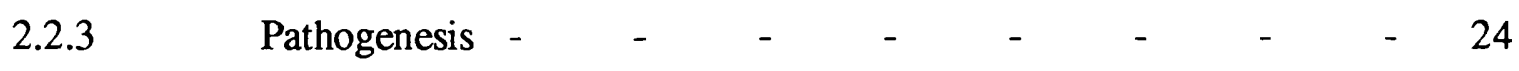

2.2.3.1 Risk factors for periodontitis 25

2.2.3.2 Dental Plaque 25

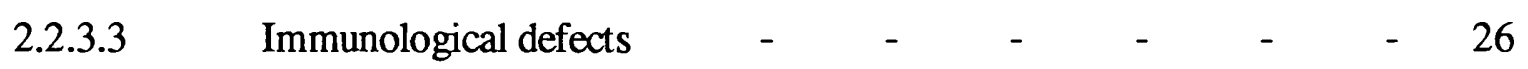

2.2.3.4 Lifestyle 29

2.2.3.5 Age 31

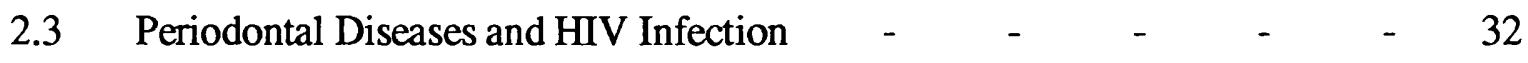

2.3.1 Periodontal Diseases seen in HIV infection 33

2.3.2 Prevalence and incidence of Periodontal Diseases seen in HIV infection 40

2.3.3 Pathogenesis of Periodontal Diseases seen in HIV infection - $\quad$ - 50

2.3.5 Management of Periodontal Diseases seen in HIV infection 58

$2.4 \quad$ Rationale for the research 58

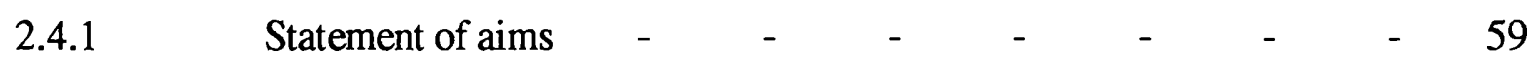




\section{Chapter 3 Methodology}

3.1 Development and reliability of diagnostic criteria for periodontal changes - $\quad 60$ associated with HIV

3.2 A cross-sectional study of the periodontal health of homosexual men with HIV 61

3.2.1 Selection of populations 61

3.3 Gingival ulceration in HIV infection: a case series and case control study 62

3.3.1 Selection of cases and controls 62

3.4 Validity of diagnostic criteria for periodontal changes associated with HIV 63

3.5 Methodological issues in epidemiology of periodontal diseases 65

3.5.1 Biological issues 65

$\begin{array}{lllllllllll}3.5 .2 & \text { Statistical issues } & - & - & - & - & - & - & - & & \end{array}$

$\begin{array}{lll}\text { 3.5.3 Measurement } & 68\end{array}$

\section{CHAPTER 4 DEVELOPMENT AND RELIABILITY OF DIAGNOSTIC CRITERIA FOR}

\section{PERIODONTAL CHANGES ASSOCIATED WITH HIV INFECTION}

4.1 Abstract 71

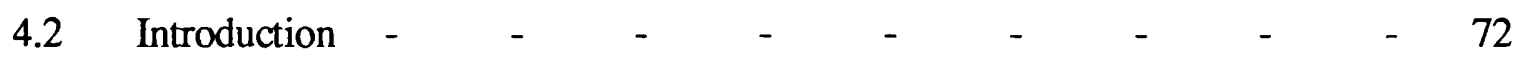

$\begin{array}{lll}4.3 & \text { Methodology } & 72\end{array}$

$\begin{array}{lll}\text { 4.3.1 Development of the criteria } & 72\end{array}$

4.3.2 Assessment of the reliability of the criteria $\quad-\quad{ }_{2} \quad-\quad-73$

$\begin{array}{lll}\text { 4.3.3 Selection of populations } & 73\end{array}$

$\begin{array}{lll}4.3 .4 & \text { Variables } & 73\end{array}$

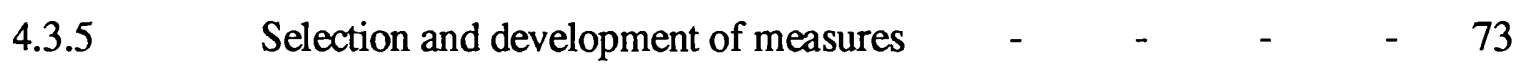

$\begin{array}{lll}\text { 4.3.6 Data collection - Conduct } & 77\end{array}$

4.3.7 Data analysis 77

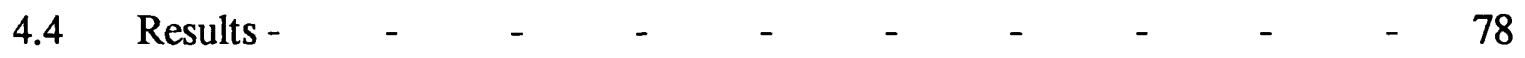

4.4.1 Signs most commonly associated with each diagnosis $\quad 78$

4.4.2 Reliability of recognition of clinical signs 81

$\begin{array}{lllllllll}\text { 4.4.3 Reliability of diagnosis } & - & - & - & - & - & - & 81\end{array}$

4.5 Discussion $\quad 82$

4.5.1 Signs associated with diagnoses $\quad 82$

$\begin{array}{llllllll}\text { 4.5.2 Reliability of recognising signs } & - & - & - & - & - & - & 84\end{array}$

4.5.3 Reliability of diagnosis 85

$\begin{array}{lll}4.6 & \text { Conclusions } & 86\end{array}$ 
Chapter 5 A Cross-Sectional STUdy OF THE PERIOdONTAL HEALTH OF HOMOSEXUAL MEN WITH HIV INFECTION

5.1 Abstract

5.2 Introduction

5.3 Method

$\begin{array}{ll}88 & 88\end{array}$

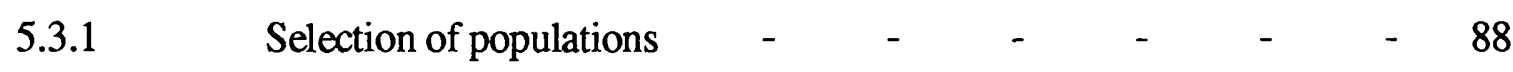

$\begin{array}{lll}\text { 5.3.2 Variables } & 89\end{array}$

$\begin{array}{lll}\text { 5.3.3 Data collection } & 94\end{array}$

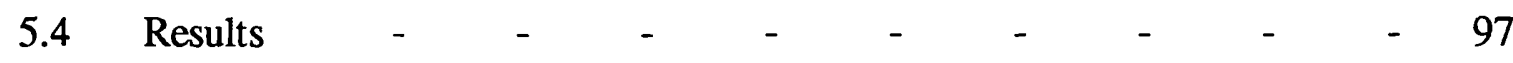

5.5 Discussion 105

$\begin{array}{lll}5.6 & \text { Conclusions } & 109\end{array}$

CHAPTER 6 GINGIVAL ULCERATION IN HIV INFECTION: A CASE SERIES AND CASE CONTROL STUDY

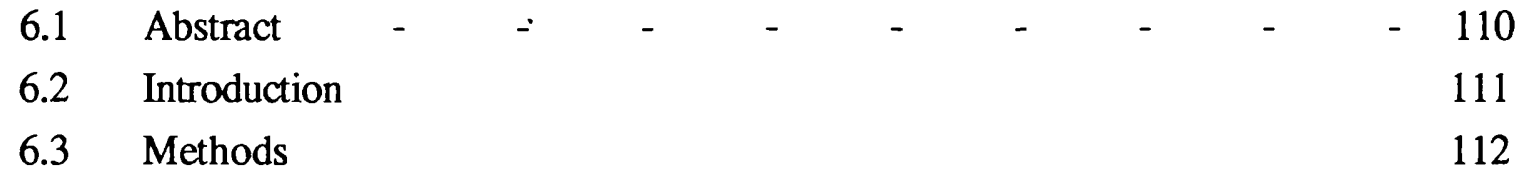

6.3.1 Description of the cases $\quad$ - $\quad$ - $\quad$ - $\quad$ - $\quad$ - $\quad$ - 112

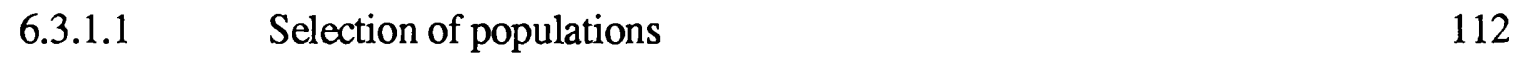

$\begin{array}{lll}\text { 6.3.1.2 Variables } & 112\end{array}$

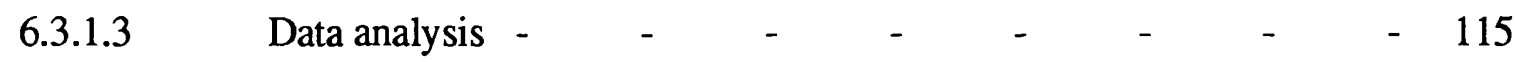

6.3.2 Cases and controls 116

$\begin{array}{lll}\text { 6.3.2.1 Data analysis } & 116\end{array}$

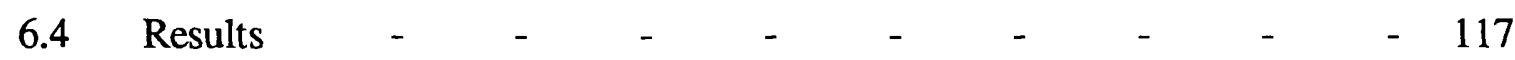

$\begin{array}{lll}\text { 6.4.1 Description of the cases } & 117\end{array}$

$\begin{array}{lll}6.4 .2 & \text { Case control study } & 126\end{array}$

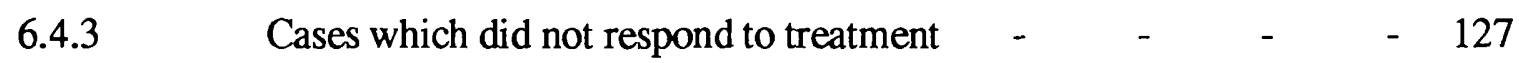

$\begin{array}{lll}6.5 & \text { Discussion } & 129\end{array}$

6.6 Conclusions 133 


\section{CHAPTER 7 THE VALIDITY OF DIAGNOSTIC CRITERIA FOR PERIODONTAL} CHANGES ASSOCIATED WITH HIV INFECTION.

\begin{tabular}{|c|c|c|c|c|c|c|c|c|}
\hline 7.1 & Abstract & - & - & - & - & - & - & - \\
\hline 7.2 & Introduction & & & & & & & \\
\hline 7.3 & Methods & & & & & & & \\
\hline 7.3.1 & Selection of population & & - & - & - & - & - & - \\
\hline 7.3 .2 & Variables - Summary & & & & & & & \\
\hline 7.3.3 & Data collection & & & & & & & \\
\hline 7.3.4 & Data analysis. - & - & - & - & - & - & - & - \\
\hline 7.4 & Results & & & & & & & \\
\hline 7.5 & Discussion & & & & & & & \\
\hline 7.6 & Conclusions & - & - & - & - & - & - & - \\
\hline
\end{tabular}

\section{CHAPTER 8 CONCLUSIONS AND RECOMMENDATIONS FOR FUTURE RESEARCH}

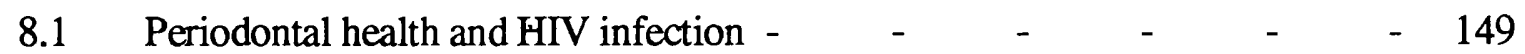

8.2 The diagnosis of periodontal changes seen in HIV infection 150

$\begin{array}{lll}8.3 & \text { Recommendations for future work } & 151\end{array}$

$\begin{array}{ll}\text { REFERENCES } & 154\end{array}$

Appendix 1 Data collection sheet in diagnostic reliability study 181

Appendix 2 Frequency of diagnoses in original, control and study groups 182

Appendix 3 Recruitment procedure in cross-sectional study 183

$\begin{array}{lll}\text { Appendix } 4 \text { Questionnaire in cross-sectional study } & 184\end{array}$

$\begin{array}{lll}\text { Appendix } 5 & \text { Sheet for medical data in cross-sectional study } & 188\end{array}$

Appendix 6 Information sheet for known HIV positive participants in cross- 189 sectional study

Appendix 7 Information sheet for participants of negative and unknown HIV 190 status in cross-sectional study

Appendix 8 Consent form for known HIV positive participants in cross- 191 sectional study

Appendix 9 Consent form for participants of negative and unknown HIV 192 status in cross-sectional study 
Appendix 10 Proportions of tooth surfaces in men with and without HIV infection with plaque scores of $0,1,2$ and 3

Appendix 11 Frequency of attachment loss of $4 \mathrm{~mm}$ or more by tobacco use in HIV-ve and HIV+ve men

Appendix 12 Severity of attachment loss by proportion of surfaces with plaque in HIV+ve and -ve men

Appendix 13 Extent of pocketing in men with and without HIV by age

Appendix 14 Prevalence of attachment loss and probing depths $\geq 4 \mathrm{~mm}$ by HIV status and CD4 count

Appendix 15 Severity of conventional gingivitis and punctate erythema of the attached gingiva by HIV status and presence of oral candidiasis

Appendix 16 Linear regression of number of ulcerated sites in first episode of 199 gingival ulceration by age

Appendix 17 Linear regression of number of ulcerated sites in first episode of gingival ulceration by neutrophil count

Appendix 18 Possible risk factors for gingival ulceration in cases \& controls

Appendix 19 Best forward stepwise regression model for gingival ulceration 202 excluding participants who were not homosexual men

Appendix 20 Diagnostic criteria for HIV associated periodontal diseases used 203 by Riley et al. (1992).

Appendix 21 Diagnostic criteria for NUG used by Thompson et al. (1992).

Appendix 22 Diagnostic criteria for HIV associated periodontal diseases of the 205 US Oral AIDS Collaborative Group (Greenspan et al, 1992).

Appendix 23 Diagnostic criteria for HIV associated periodontal diseases of the 206 EC-Clearinghouse on Oral problems related to HIV infection (1993)

Appendix 24 Diagnostic criteria for HIV associated periodontal diseases used 207 by Grbic et al. (1995)

Appendix 25 Publications, published abstracts and presentations at major 208 scientific meetings in support of this work

Appendix 26 Glossary of abbreviations 
TABLES

Table 2.1 Centers for Disease Control and Prevention classification of HIV disease

Table 2.2 Studies of the periodontal health of people taking immunosuppressive drugs

Table 2.3 Prevalence studies of periodontal health in HIV infection

Table 2.4 Longitudinal studies in people with HIV infection not receiving specialist periodontal treatment

Table 2.5 Immunohistochemical studies of immune cells in the gingiva of people with HIV

Table 4.1 Diagnostic Criteria for studies of HIV-associated periodontal changes

Table 4.2 Diagnoses for 20 clinical slides made by 9 examiners

Table 4.3 Clinical signs associated with diagnoses by 9 examiners

Table 4.4 Kappa scores for inter-rater agreement on signs of periodontal diseases

Table 5.1 Frequency of inhaled drug use among 312 men with and 260 without HIV infection

Table 5.2 The presence of periodontal changes in homosexual men by HIV status

Table 5.3 Best forward stepwise multiple and logistic regression models for outcomes related to loss of periodontal attachment in 312 men with and 260 without HIV infection

Table 5.4 Best forward stepwise multiple and logistic regression models for gingival health outcomes in 312 men with and 260 without HIV infection

Table 5.5 Best forward stepwise multiple and logistic regression models for outcomes related to loss of periodontal attachment in 312 men with HIV infection

Table 5.6 Best forward stepwise multiple and logistic regression models for gingival health outcomes in 312 men with HIV infection 
Table 6.1 Frequency distribution for number of episodes among 89 patients with gingival ulceration

Table 6.2 Independent variables in mild and severe episodes of gingival ulceration

Table 6.3 Independent variables in cases of gingival ulceration who did and did not relapse

Table 6.4 Median numbers of sites ulcerated by severity of HIV disease, oral diseases and current tobacco use

Table 6.5 Best forward stepwise logistic regression model for independent variables related to the presence of gingival ulceration among 89 cases and 269 controls

Table 6.6 Best forward stepwise regression model for gingival ulceration among 279 patients not taking co-trimoxazole

Table 7.1 Possible equivalents in eight sets of diagnostic criteria of HIVassociated periodontal changes.

Table 7.2 Validity of 5 diagnostic criteria for ulceration of the marginal gingiva associated with HIV infection

Table 7.3 Validity of 5 diagnostic criteria for extensive periodontal ulceration associated with HIV infection.

Table 7.4 Validity of 5 diagnostic criteria for linear gingival erythema/HIVassociated gingivitis.

Table 7.5 Validity of 6 signs of linear gingival erythema/HIV-associated gingivitis.

Table 7.6 Validity of 3 tests for lost periodontal attachment associated with HIV infection (Crude data)

Table 7.7 Validity at sites with lost periodontal attachment in HIV infection (Adjusted data)

Table 7.8 Operational criteria for periodontal changes associated with HIV infection

Appendix 18 Possible risk factors for gingival ulceration in cases and controls

Appendix 19 Best forward stepwise regression model for gingival ulceration 202 excluding participants who were not homosexual men 


\section{FIGURES}

$\begin{array}{lll}\text { Figure 2.1 Risk factors for periodontitis } & 25\end{array}$

Figure 2.2 Distinct red gingival bands in a 13 year old haemophiliac boy with 34 AIDS

Figure 2.3 Punctate erythema of the attached gingiva of a homosexual man with HIV

Figure 2.4 Ulceration localised to the gingival margin and diffuse erythema of the attached gingiva in a homosexual man with HIV infection

Figure 2.5 Extensive ulceration and necrosis in a 47 year old homosexual man with AIDS

Figure 4.1 Decision making flow chart for diagnosis of periodontal conditions associated with HIV

Figure 6.1 Gingival ulceration in a 47 year old bisexual man with HIV infection

Figure 6.2 Frequency distribution for the number of sites affected in first episode of gingival ulceration

Figure 6.3 Frequency distribution of teeth affected in first episode of gingival ulceration, corrected for teeth present.

Figure 6.4 Lesion in Figure 6.1 one week after debridement. Sequestrum is visible.

Figure 6.5 Same site as Figures 6.1 and 6.4 two years later. The probe has Williams markings and the tip can be moved between the two teeth $5 \mathrm{~mm}$ from the cemento-enamel junction.

Figure 6.6 Relative frequency of gingival ulceration among patients with AIDS using and not using co-trimoxazole and patients with early HIV disease

Appendix 2 Frequency of diagnoses in original, control and study groups

Appendix 10 Proportions of tooth surfaces in men with and without HIV infection with plaque scores of $0,1,2$ and 3

Appendix 11 Frequency of attachment loss of $4 \mathrm{~mm}$ or more by tobacco use in HIV-ve and HIV+ve men 
Appendix 12 Severity of attachment loss by proportion of surfaces with plaque in HIV+ve and -ve men

Appendix 13 Extent of pocketing in men with and without HIV infection by age

Appendix 14 Prevalence of attachment loss and probing depths $\geq 4 \mathrm{~mm}$ by HIV status and CD4 count

Appendix 15 Severity of conventional gingivitis and punctate erythema of the attached gingiva by HIV status and presence of oral candidiasis

Appendix 16 Linear regression of number of ulcerated sites in first episode of gingival ulceration by age

Appendix 17 Linear regression of number of ulcerated sites in first episode of gingival ulceration by neutrophil count 


\section{CHAPTER 1 INTRODUCTION AND OVERVIEW OF THE THESIS}

Detailed research on periodontal changes seen in HIV disease has produced conflicting results, mainly on the nature and frequency of these conditions. This thesis describes four studies of the occurrence of these changes undertaken with regard to knowledge of HIV disease, periodontology and epidemiological methodology.

Chapter 2 presents a background for the thesis. HIV disease, its pathogenesis and its oral manifestations are discussed. Periodontal diseases in conventional populations are considered with emphasis on variables relevant to the relationship between HIV and periodontal health. Since HIV affects the immune system, some consideration is given to immunological factors in periodontal diseases. The literature on HIV and periodontal health is reviewed in detail. The section ends with the rationale for the research and a statement of the aims.

Chapter 3 summarises epidemiological methodology and discusses some methodological issues in periodontology. The specific methods used in each study are detailed in individual reports in the four following chapters

The 4th. chapter describes the development and reliability tests of diagnostic criteria for HIV-associated periodontal changes. The way in which dental experts in HIV diagnosed the changes was also investigated.

Chapter 5 reports on a controlled blinded cross-sectional study of homosexual men attending a genito-urinary medicine clinic to identify the types, prevalence and severity of periodontal changes seen in HIV infection. Some changes were uncommon, so Chapter 6 describes a case series and case controlled study of gingival ulceration among patients attending a dedicated dental clinic.

The study reported in Chapter 7 compared the validity of diagnostic criteria for HIVassociated periodontal changes.

Conclusions from the research are summarised and avenues for future research are discussed in Chapter 8. 


\section{CHAPTER 2 BACKGROUND AND REVIEW OF THE LITERATURE}

This chapter reviews information pertinent to research of periodontal health in HIV disease. First, HIV disease, its pathogenesis and its oral manifestations are discussed. Secondly, periodontal diseases in conventional populations are considered with emphasis on factors which might mediate, confound or interact in the relationship between HIV and periodontal health. Since HIV affects the immune system, brief consideration is given to immunological factors in periodontal diseases. Thirdly, the literature on HIV and periodontal health is reviewed in detail. The section ends with the rationale for the research and a statement of the aims.

\subsection{HIV and AIDS}

Human immunodeficiency virus (HIV) is a retrovirus which causes progressive impairment of the regulation and function of the immune system. The final stage of infection, acquired immunedeficiency syndrome (AIDS) is invariably fatal.

\subsubsection{Epidemiology}

HIV is transmitted in body fluids, most notably blood products, semen, cervical secretions and occasionally breast milk (Lifson, 1992). Epidemics of HIV infection therefore occur where there is frequent exchange of fluids. In developed countries the majority of cases are among homosexual men and injecting drug users with an increasing heterosexual component. Mother-to-child transmission is uncommon due to the high male:female ratio of infection. The World Health Organisation (WHO) terms this 'Pattern 1' spread (Mann et al, 1988).

The distribution of HIV in the United Kingdom (UK) is characteristic of this pattern. At the end of February 1996, the cumulative total of AIDS cases was 12201 of whom $90.4 \%$ were men (Centre for Disease Surveillance and Control, 1996). 8493 (69.6\%) had died. Data on HIV infection are available to the end of December 1995. The cumulative total of people infected was 25685 of whom 4651 (18.1\%) had been infected heterosexually.

'Pattern 2' relates to sub-Saharan Africa and parts of the Caribbean where transmission is mainly heterosexual. 'Pattern 3' describes the situation in Eastern Europe, the Middle East and Asian and the Pacific countries where incidence has been low but may increase, especially where sex industries and tourism are related. 
The pathogenesis of HIV is based on its interaction with host CD4+ve T lymphocytes. Viral envelope protein gp120 binds to the CD4 membrane receptor which allows the virion to fuse to and enter the cell. The virion is uncoated and a single strand of complementary DNA is generated from the HIV RNA using reverse transcriptase. A second DNA strand is synthesised to produce a double stranded DNA copy of the original HIV RNA. This is translocated to the nucleus and inserted onto the host chromosome (Connor and Ho, 1992). HIV RNA is then replicated and there is a cytopathic effect on the host cell.

CD4+ve T-lymphocytes co-ordinate many functions of the immune system including those mediated by the B cells, monocyte-macrophages, cytotoxic $\mathrm{T}$ cells, suppressor $\mathrm{T}$ cells and natural killer cells. In addition to CD4 cell depletion, infection causes functional defects in the cells. They respond abnormally to a range of soluble antigens and have altered receptors for and production of cytokines (Rosenberg and Fauci, 1992).

Other cells susceptible to HIV infection include monocyte/macrophages, bone marrow CD34+ve precursor cells, monocytic and T-cell lines, epithelial Langehans cells, cells of the central nervous system, retina, cervix and colon. HIV does not enter cells without CD4 expression readily in vitro and the mechanisms allowing infection of these cells are unknown (Connor and Ho, 1992). Other cells are indirectly affected by HIV.

Approximately $8 \%$ of people with asymptomatic HIV infection are neutropenic (Zon and Groopman, 1988) either due to ineffective neutrophil production, neutrophil antibodies or medications (McCance-Katz et al, 1987; Israel and Plaisance, 1991). There is also a consensus that neutrophil activity is impaired through a combination of decreased random migration, chemotaxis, adhesion, bacterial killing and phagocytosis (Ras et al, 1984; McCance-Katz et al, 1987; Ellis et al, 1988; Elbim et al, 1994; Flø et al, 1994) which are present in early HIV infection and pronounced in later disease (Roilides et al, 1993; Flø et al, 1994).

Ryder et al. (1988) detected elevated phagocytosis, oxidative burst, and F actin formation in neutrophilic polymorphonuclear leukocytes (PMN's) from patients with oral candidiasis and HIV-associated periodontitis. This group suggested that their findings differed from earlier work because they had studied exclusively homosexual men with oral disease. Earlier research had found decreased PMN migration among patients with HIV whether or not they were drug users (Lazzarin et al, 1986). Elbim et al. (1994) suggested different research methods accounted for variation in findings. They studied neutrophils in whole 
blood and detected activated PMN function in patients with HIV until the neutrophils were primed with cytokines and stimulated with bacterial peptides when their function was dysregulated and decreased.

Neutrophil dysfunction may be mediated through serum cytokines (e.g. TNF alpha), immuno-complexes and viral proteins since the serum from patients with HIV inhibits neutrophils from healthy controls (Ras et al, 1984; Roilides $e t$ al, 1993; Elbim et al, 1994; Flø et al, 1994). Three medications used in HIV disease: Zidovudine; fluconazole and cotrimoxazole do not impair neutrophil function (Flø et al, 1994).

The cumulative effect of neutrophil impairment, neutropenia and other defects of the cell mediated and humoral immune systems may increase susceptibility to bacterial and fungal infections and may allow fungal infections with milder neutropenia than seen in otherwise healthy patients (Roilides et al, 1993; Elbim et al, 1994; Flø et al, 1994).

\subsubsection{Clinical presentation.}

The immunodeficiency of HIV infection is manifest by a range of opportunistic infections (often viral or fungal because of the impaired cell mediated immunity), auto-immune conditions and tumours. The United States (US) Centers for Disease Control and Prevention (CDC)(1992) recognises 3 clinical categories of HIV disease (Table 2.1). Category A disease represents early disease including primary infection, asymptomatic infection and prolonged generalised lymphadenopathy. Category $\mathrm{C}$ disease is defined by the presence of one of 26 AIDS indicator conditions and HIV infection. The most common indicators for AIDS in the UK are Pneumocystis carinii pneumonia (40\% of cases) and Kaposi's sarcoma (14\%) (Centre for Disease Surveillance and Control, 1995). Category B comprises patients with symptomatic disease who do not have AIDS.

The CD4+ve lymphocyte count (CD4 count) is an haematological measure and prognosticator of HIV disease progression (Lang et al, 1989; Fahey et al, 1990; Centers for Disease Control and Prevention, 1992). Three categories based on CD4 counts are included in the CDC (1992) classification. Categories 1, 2 and 3 represent CD4 counts of more than 500 cells $/ \mathrm{mm}^{3}$, between 200 and 500 cells $/ \mathrm{mm}^{3}$ and less than 200 cells $/ \mathrm{mm}^{3}$ respectively. The CDC case definition for AIDS includes people with CD4 counts less than 200 cells $/ \mathrm{mm}^{3}$. This criterion is not used for surveillance in the UK. 
Table 2.1 Centers for Disease Control and Prevention classification of HIV disease (Centers for Disease Control and Prevention, 1992)

\begin{tabular}{|c|c|c|c|c|}
\hline & \multicolumn{3}{|c|}{ Clinical Categories } \\
\hline & & $\mathbf{A}$ & B & C \\
\hline & $\begin{array}{l}\text { CD4+ve cell } \\
\text { categories } \\
\text { (cells/mm } 3 \text { ) }\end{array}$ & $\begin{array}{l}\text { Asymptomatic, } \\
\text { acute HIV or PGL }\end{array}$ & $\begin{array}{l}\text { Symptomatic, not } \mathrm{A} \\
\text { or } \mathrm{C} \text { conditions }\end{array}$ & $\begin{array}{l}\text { AIDS indicator } \\
\text { conditions }\end{array}$ \\
\hline 1 & $>500$ & Al & B1 & $\mathrm{C} 1$ \\
\hline 2 & $200-500$ & A2 & B2 & $\mathrm{C} 2$ \\
\hline 3 & $<200$ & A3 & B3 & C3 \\
\hline
\end{tabular}

The grey areas are those which meet the case definition for AIDS.

\subsubsection{Oral Manifestations of HIV infection}

A number of oral changes are seen in HIV infection (Greenspan et al, 1990). Some associations are based on individual cases and consequently oral diseases have been classified by the strength of their association with HIV (EC-Clearinghouse, 1993). The diseases most strongly associated with HIV are oral candidiasis, oral hairy leukoplakia, linear gingival erythema, necrotising ulcerative gingivitis, necrotising ulcerative periodontitis, Kaposi's sarcoma and non-Hodgkin's lymphoma. Salivary gland disease is less commonly associated with HIV infection but of possible significance to the pathogenesis of periodontal diseases since it may cause xerostomia. Periodontal diseases seen in HIV infection are reviewed in Section 2.3

\subsubsection{Oral Candidiasis}

Whilst oral carriage of Candida spp. is common in conventional populations, infection only occurs in the presence of predisposing factors. Local factors include denture wearing, xerostomia and topically applied medications. Systemic factors include pregnancy; iron and vitamin deficiencies, use of broad-spectrum antibiotics and HIV infection (Odds, 1988). Among non-HIV infected populations 5 clinical forms are recognised: Acute pseudomembranous; acute atrophic; chronic atrophic; chronic hyperplastic and angular cheilitis (Lynch, 1994).

Three presentations are recognised in HIV infection: pseudomembranous; erythematous and angular cheilitis (EC-Clearinghouse, 1993). 
Greenspan et al. (1990) found the prevalence of oral candidiasis ranged between $43 \%$ and 93\% in studies of people with advanced HIV disease. Disease progression is associated with increased carriage of and infection with Candida spp. In 2 independent studies carriage of Candida albicans rose from $57.5 \%$ to $87.5 \%$ between CDC stages 1 and 3 and the prevalence of candidiasis rose from $14 \%$ to $26 \%$ between the 2 nd and 5 th years of infection (Korting et al, 1989; Lifson et al, 1994). Oral candidiasis is therefore a prognosticator of progression to AIDS with a relative hazard of 7.3 compared to people with HIV and no oral disease (Katz et al, 1992). Dodd et al. (1991) found similar rates of progression for erythematous and pseudomembranous candidiasis but, selection, recall and diagnostic bias undermine this study.

\subsubsection{Oral Hairy Leukoplakia}

Oral hairy leukoplakia $(\mathrm{OHL})$ is a white hyperkeratotic lesion which usually occurs on the lateral borders of the tongue (Greenspan and Greenspan, 1992). Lesions are variable in appearance and usually asymptomatic. Classically, they are corrugated from the dorsal to ventral surface but are often irregular and occasionally hairy. The hyperkeratosis is an expression of Epstein-Barr Virus (EBV) infection. EBV should be demonstrated in OHL using immunocytochemistry or molecular biology to confirm diagnosis (Greenspan et al, 1992).

Although case reports exist of OHL among people receiving immuno-suppressive treatment following transplants (Greenspan and Greenspan, 1992), it is almost pathognomic of HIV infection. The prevalence of OHL in HIV infection is approximately $20 \%$, with a range of 5\%-36\% (Phelan et al, 1987; Schiodt et al, 1990). OHL is a prognosticator for progression to AIDS with a relative hazard of 1.8 compared to people with no oral disease. (Katz et al, 1992)

\subsubsection{Kaposi's Sarcoma}

Kaposi's Sarcoma (KS) is a multi-focal neoplastic proliferation of endothelial cells and fibroblasts originally described in elderly eastem European men and subsequently in black Africans and people receiving immuno-suppressive treatment (Kaposi, 1872; Epstein and Silverman, 1992). KS is uncommon in Western Europe and North America unless associated with HIV infection in which case it is one of the diagnostic conditions for AIDS.

Whilst $21 \%$ of homosexual and bisexual men with AIDS have KS (Beral $e t$ al, 1990) the prevalence is much lower among other groups. This led researchers to suspect a sexually- 
transmitted co-factor in its pathogenesis (Epstein and Silverman, 1992) and a new Herpes virus has been identified as a causative agent (Chang et al, 1994). Oral KS initially manifests as purple-red-blue macules. Advanced lesions may be raised and ulcerated with trauma. Lesions are often asymptomatic and require no treatment. Bulky lesions can be treated by surgical excision, radiotherapy, systemic or intralesional chemotherapy and meticulous dental hygiene if adjacent to the teeth (Epstein and Silverman, 1992; Shiboski and Winkler, 1993).

\subsubsection{Non-Hodgkin's lymphoma}

An increased incidence of non-Hodgkin's lymphoma (NHL) in homosexual men was reported in 1982 (Lozada et al, 1982). NHL in the presence of HIV is diagnostic of AIDS. The disease is usually of B cell origin, is often aggressive and carries a poor prognosis (Epstein and Silverman, 1992). Oral lesions usually present as soft tissue tumefaction but are uncommon and appear as isolated cases in prevalence studies (Porter and Scully, 1994).

\subsubsection{Salivary gland disease}

HIV-associated salivary gland disease (HIV-SGD) is defined as 'xerostomia, enlargement of one or more of the major salivary glands, or both' in people with HIV (Schiodt, 1992). This definition includes neoplasia such as $\mathrm{KS}$ and 10 non-neoplastic changes ranging from cystic lymphoid hyperplasia to a Sjogrens syndrome-like illness. It is likely that these overlap as different presentations of the same condition (Challacombe and Schiodt, 1995).

The pathogenesis of HIV-SGD is unknown, partly because the disease is uncommon among adults. In three studies totalling 944 adults with HIV (Colebunders $e t$ al, 1988; Langford et al, 1988; Schiodt, 1992), only 4 had parotid swelling (0.4\%), although $10 \%$ of subjects with or at risk of HIV complained of dry mouth in one study (Silverman et al, 1986). The prevalence is higher among children with HIV, where up to $7 \%$ have parotid swelling (European Collaborative Study, 1991).

In a review of salivary gland involvement in HIV infection, Fox identified a consistent decrease in salivary flow, especially in stimulated submandibular and sublingual secretion (Fox, 1992). A range of salivary compositional changes have also been described. The most consistent of these include elevated chloride, lysozyme, total protein and IgA levels. 


\section{$\underline{2.2 \quad \text { Periodontal Diseases }}$}

Diseases of the periodontium are classified by the position of the junctional epithelium (Caton, 1989). In gingivitis the apical portion of the junctional epithelium remains near the cemento-enamel junction. In periodontitis apical migration of the junctional epithelium reduces periodontal ligament attachment and even bone support.

\subsubsection{Gingivitis}

There are two forms of gingivitis, chronic marginal gingivitis and acute necrotising ulcerative gingivitis. Systemic involvement including hormonal changes, skin diseases, generalised systemic diseases, infective gingivostomatitis and drugs may modify these diseases or cause other gingival changes.

\subsubsection{Chronic marginal gingivitis}

Gingivitis is characterised by the rubor and tumour of inflammation and by bleeding. The swelling changes the surface texture of the tissue resulting in loss of stippling and the free gingival margin. Bleeding may be spontaneous or with gentle probing or brushing. Pain is an uncommon feature.

The disease is an inflammatory response to plaque and is exceedingly common. Bleeding on probing is present in $79 \%$ of UK adults and $85 \%$ in the US (Brown et al, 1989; Todd and Lader, 1991). Erythematous changes are likely to be more common.

\subsubsection{Acute Necrotising Ulcerative Gingivitis (ANUG)}

ANUG is characterised by interproximal necrosis, ulceration, soreness, blunting of the interdental papillae and gingival bleeding (Johnson and Engel, 1986). The ulcerated papillae may have a grey pseudomembrane of slough and there may be a characteristic foetor of 'wet hay'. Lymphadenopathy and mild fever are variable findings (Stammers, 1944; Goldhaber and Giddon, 1964).

The disease can be progressive and in the absence of treatment may not be self-limiting (Johnson and Engel, 1986). In severe cases necrosis may extend over adjacent and contiguous tissues to cause gross destruction of oral and facial tissues. This has been called cancrum oris or noma (Emslie, 1963). 
There are five related aetiological factors for ANUG: plaque accumulation, infection, tobacco use, stress and immunological changes (Schluger, 1943; Barnes et al, 1973; Johnson and Engel, 1986). Infection was implicated in ANUG as early as 1890 when Vincent identified a fusiform and a spirochaete ('Bacillus vincentii' and 'Borrelia vincentii') in samples from lesions. Electron microscopy revealed 4 zones in the lesions: 1 , a bacterial zone; 2 , a neutrophil rich zone; 3 , a necrotic zone and 4 , a zone of infiltration by spirochaetes (Listgarten, 1965; Courtois et al, 1983). Defining the microbial aetiology of periodontal diseases is complicated by conflicting findings from studies using different methodologies and because no micro-organisms are specific to healthy or diseased sites. A consensus on the microbiology of ANUG implicates a complex of Borelia vincentii, Fusobacterium nucleatum (formerly Fusiform fusiformis) and a black-pigmented anaerobic Gram negative rod Prevotella intermedia (previously Bacteroides melaninogenicus spp. intermedius) (Loesche et al, 1982). Earlier work in which experimental animals were inoculated with cultured organisms has placed greater emphasis on the $P$. intermedia (MacDonald et al, 1956; MacDonald et al, 1960) or, conversely the fuso-spirochaetal complex (Rosebury et al, 1950; Hampp and Mergenhagen, 1961; Mikx and van Campen, 1982).

Tobacco smoking has long been implicated in the aetiology of ANUG (Goldhaber and Giddon, 1964) and is universally accepted to predispose to the condition. Early studies were rarely controlled and the confounding effects of smoking and poor plaque control were not considered but the evidence for the role of smoking in the aetiology of ANUG is compelling. Fifty-six of 57 new cases of ANUG seen by Pindborg (1951) were in smokers, the incidence of ANUG among students who smoked was double that among non-smokers and in a series of 72 German men with ANUG, three quarters smoked more than 60 cigarettes daily (Giddon et al, 1964; Pollmann and Dietrich, 1979).

Immunological changes are implicated in the pathogenesis of ANUG. Listgarten et al. (1965) described a neutrophil rich zone in ANUG lesions. Conversely, Courtois et al. (1983) found a predominance of lymphocytes and plasma cells in some lesions although other lesions had the PMNs described by Listgarten. These features may have been causative or reactive. Cogen et al. (1983) compared 35 patients with ANUG against 35 age, sex and race matched controls. There was reduced peripheral PMN proliferation and chemotaxis in response to the plant glycoprotein Concanalvin A (Con A). Claffey et al. (1986) also found PMN chemotaxis was decreased in 11 patients with ANUG compared to 11 controls. PMN function had returned to normal levels in 4 patients who were retested 18 months later. It was suggested that ANUG was related to transient defects in PMN function and that PMN proliferation and chemotaxis mediated the effects of stress in the disease. 
Interestingly, decreased CD4 counts and CD4:CD8 ratios were noted in 5 homosexual men with ANUG (Dennison et al, 1985). This was the first report to associate periodontal changes with HIV infection. These men have since been assumed to have 'HIV-associated periodontitis' or 'necrotising ulcerative periodontitis' (Winkler et al, 1988; ECClearinghouse, 1993). However, the report exists only as an abstract and cannot be evaluated fully. Possible differences between ANUG and HIV-associated periodontitis are discussed in Section 2.3.

The role of stress in the aetiology of ANUG is further considered below.

\section{$\mathbf{2 . 2 . 2}$}

\section{Periodontitis}

Periodontal attachment loss is manifest by periodontal pockets and/or recession. Pockets are formed if the junctional epithelium migrates apically more than the crestal gingiva. In recession there is loss of crestal tissue which exposes the underlying root surfaces.

The American Academy of Periodontology (Caton, 1989) classifies periodontitis into 4 categories: periodontitis in adults, periodontitis in juveniles, periodontitis with systemic involvement (especially with primary and secondary neutrophil disorders) and other conditions affecting the periodontium. Adult periodontitis is the most common and is further categorised by its rate of progression (e.g. 'rapidly progressing periodontitis'), response to treatment ('refractory' or 'recurrent') or history (e.g. 'post recurrent acute necrotising ulcerative periodontitis').

Rapidly progressive periodontitis (RPP) is a distinct clinical condition with rapid alveolar destruction and pronounced inflammation and haemorrhage of the gingiva, sometimes with a systemic illness (Page et al, 1983). In their series of seven patients the molar teeth were often affected. Most patients had altered neutrophil or monocyte chemotaxis and the disease was likened to other periodontal diseases associated with neutrophil disorders.

The frequency of periodontitis is difficult to assess. The unusual forms of periodontitis usually appear as case reports rather than in epidemiological studies. In addition, concepts of destructive periodontal disease introduced in 1984 suggest reduced periodontal attachment may not denote active disease (Socransky et al, 1984). If there are bursts of destruction followed by quiescence then lost attachment may be the legacy of previous disease and more sophisticated tests are required to detect 'active' disease. 
The prevalence of pocketing in UK and US adults is $69 \%$ and $50 \%$ respectively (Brown $e t$ al, 1989; Todd and Lader, 1991) but evidence of severe periodontitis is less frequent. Less than $8 \%$ of employed adults in the US have one or more sites with $6 \mathrm{~mm}$ lost attachment and a similar proportion of UK adults have pockets of $6 \mathrm{~mm}$ (Miller $e t \mathrm{al}, 1987$; Todd and Lader, 1991). Löe et al. (1986) identified a sub-group of Sri Lankans comprising approximately $8 \%$ of a sample of tea workers with rapidly progressive periodontitis (this is not the distinct RPP described by Page et al. (1983)).

These findings suggest that the cumulative experience of destructive periodontal disease is approximately 8 to $10 \%$ of which adult periodontitis is the most common. This figure is relatively constant between different study populations which suggests an immunogenetic susceptibility to periodontitis.

\subsubsection{Pathogenesis}

The pathogenesis of periodontitis involves the interaction of plaque periodontopathogens with the host's immune system (Genco and Slots, 1984). Periodontal destruction occurs directly as a result of pathogenic bacterial components and indirectly via host destructive mechanisms that are part of the immune response to infection (See Genco (1990) for a review). Direct effects are caused by bacterial histolytic enzymes, endotoxins, exotoxins and factors which are not toxic but which interfere with cell function. Indirect tissue destruction results from induction, stimulation or activation of the host immune system. There is modified fibroblast growth and collagen synthesis, macrophages are activated (with associated collagenase release) as are lymphocytes. Bone resorption may be stimulated by products of mononuclear cells such as prostaglandins and tumour necrosis factor.

Host and environmental risk factors modify the interaction between pathogen and host (Hart et al, 1994). A broader model (Figure 2.1) shares the biological features of the conventional models but incorporates environmental pressures outside the host which act on the agent (social pressure for oral hygiene) (Clarke and Hirsch, 1995). This model will be used as a framework to describe risk factors in detail. 
Figure 2.1 Risk factors for periodontitis (After Clarke and Hirsch, 1995)

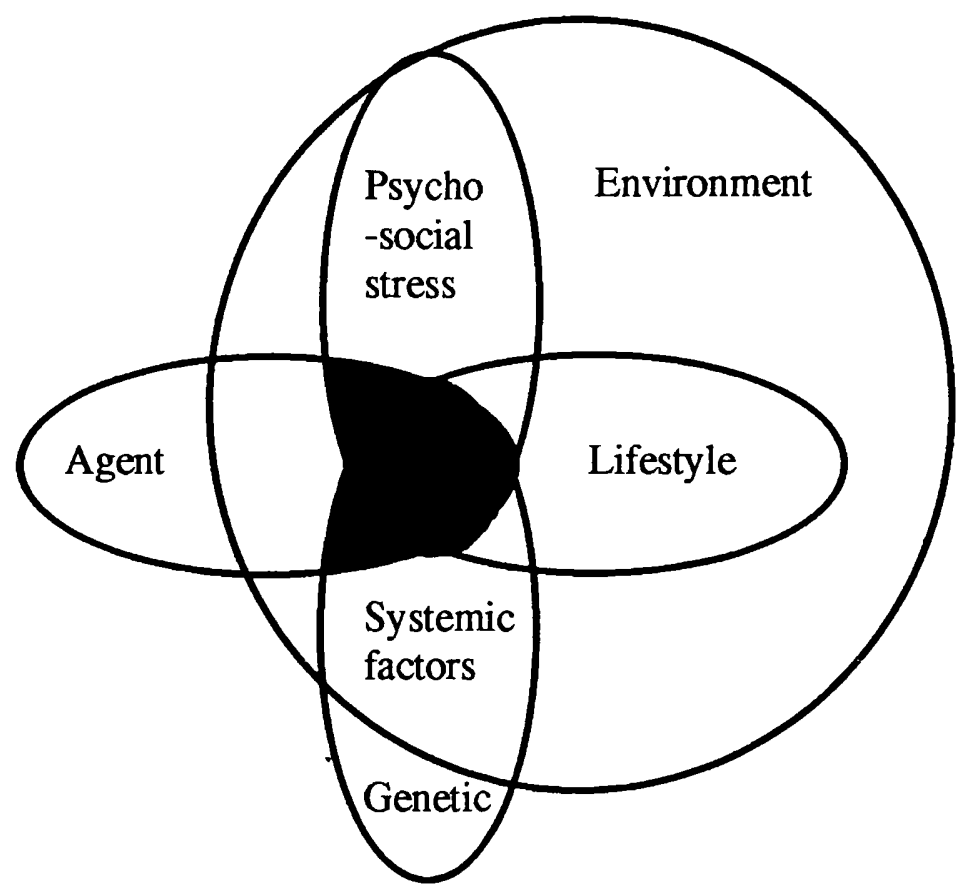

\subsubsection{1 $\quad$ Risk factors for periodontitis}

\subsubsection{Dental Plaque.}

Dental plaques are consistently implicated in the aetiology of periodontal diseases. In clinical studies gingivitis is initiated by plaque (Silness and Löe, 1964; Löe et al, 1965) and reduced by its mechanical and chemical suppression (Löe and Schiott, 1970). Löe and Morrison (1990) thought the association between plaque and gingivitis so strong that research needed to account for plaque before investigating the effect of other factors.

Plaque is also implicated in periodontitis (Lovdal et al, 1958). Progression of periodontal destruction is reduced with controlled oral hygiene (Suomi et al, 1971; Axelsson and Lindhe, 1978) and plaque bacteria initiate and enhance alveolar destruction in animal studies (Gibbons and Socransky, 1966; Holt et al, 1988). Slots and Genco (1984) have reviewed virulence factors of periodontal pathogens which include endo- and exotoxins.

Considerable research is devoted to determining which, if any, specific pathogens are responsible for periodontal attachment loss (The 'specific plaque hypothesis'. See Christersson et al. (1991) for a review). However, King (1972) distinguished necessary and sufficient causes for a disease. Dental plaque, which is necessary for disease, is ubiquitous. Since periodontal attachment loss is uncommon, plaque is not sufficient cause 
for disease. It may be more important to determine who gets the disease than which bacteria are implicated (Kass, 1970). Clarke and Hirsch (1995) concluded that microbiological research had diverted attention from the determinants of periodontal disease susceptibility.

\subsubsection{Immunological defects}

Study of periodontal health in people with impaired immune systems has shed light on the role of the immune system in the disease (Genco and Slots, 1984; Hart et al, 1994). The protective role of the neutrophil is demonstrated by the recurrent oral ulceration, marked gingivitis and periodontitis seen in primary neutrophil disorders (E.g. Agranulocytosis, cyclic neutropenia, Chediak-Higashi syndrome, neutrophil adherence abnormalities, hyperimmunoglobulinaemia E, Lazy leukocyte syndrome and neutrophil functional abnormalities) (Cohen and Morris, 1961; Deasy et al, 1980; Baehni et al, 1983 ; Charon et al, 1985; Lamster et al, 1987) or those secondary to systemic disease (Diabetes mellitus, Down's syndrome, Papillon-LeFebvre syndrome, Crohn's disease, Addison's disease and acute monocytic leukaemia) (Cianciola et al, 1982). Porter and Scully (1993) reported a series of 17 children (aged 2-16) referred to an oral medicine clinic with chemotactic or phagocytic defects of PMNs and found none had periodontal destruction. No data were presented on their gingival health but $6(35 \%)$ had oral ulceration.

The role of lymphocytes in periodontal diseases has been investigated in the last 2 decades and data on cells extracted from human periodontitis lesions suggest imbalances in local immuno-regulation (Seymour, 1987). Research of both human and animal models has resulted in a consensus that gingivitis and periodontitis lesions can be distinguished immunohistologically by the predominately $\mathrm{T}$ lymphocyte infiltrate of gingivitis and the $\mathrm{B}$ cell nature of periodontitis (see Seymour (1987) for a review).

Subsequent immunohistology of the subpopulations of periodontal and peripheral $T$ lymphocytes found in differing states of periodontal health has produced two groups of findings. A number of studies have found fewer CD4 lymphocytes or reduced CD4:CD8 ratios of approximately $1: 1$ in periodontitis sites when compared with healthy sites or peripheral blood where the ratios are approximately 2:1 (Taubman et al, 1984; Johannessen et al, 1986; Reinhardt et al, 1988; Sohoel et al, 1992). There have also been reports of lower CD4:CD8 ratios among peripheral blood lymphocytes (PBLs) in early-onset and refractory periodontitis (Kinane et al, 1989; Hernichel-Gorbach et al, 1994).

CD4:CD8 ratios in periodontitis which were similar to or higher than normal levels have also been observed (Okada et al, 1983; Syrjänen et al, 1984; Meng and Zheng, 1990; 
Malberg et al, 1992). One hypothesis suggests that fewer CD8 suppressor cells allows proliferation of B lymphocytes which damage the tissues. Different research methods may explain the contrasting observations. Samples may have been taken at different stages of periodontal destruction and various groups have studied people with adult periodontitis, diabetes associated periodontitis and localised juvenile periodontitis. Thus the significance of CD4:CD8 lymphocyte ratios in periodontitis remains unclear. The picture is further complicated by research of CD4 lymphocyte subsets and changes in lymphocyte function in periodontitis (see Seymour, (1987) for a review).

Epidemiological research of the role of lymphocytes in periodontal diseases has been difficult. Children with lymphocyte disorders rarely survive long enough to allow study of their periodontal health. Porter and Scully (1993) reported a series of 10 children (aged 3 to 17) with $T$ lymphocyte defects. Unfortunately, full periodontal assessments were not performed and only one child was diagnosed with 'gingivitis'.

Until recently the only other groups of people with lymphocyte disorders available to participate in research were those taking azathioprine and/or prednisolone, either for immunosuppression to prevent renal allograft rejection (Schuller et al, 1973; Kardachi and Newcomb, 1978; Oshrain et al, 1979; Been and Engel, 1982; Oshrain et al, 1983; Sutton and Smales, 1983; Tollefsen and Johansen, 1985; Seymour et al, 1987), management of multiple sclerosis (Safkan and Knuuttila, 1984) or pemphigus vulgaris (Markitziu et al, 1990). A combination of these drugs inhibits lymphocyte production, depresses lymphocyte function and is lymphocytotoxic (Jasani, 1979). T lymphocytes are also selectively inhibited by azathioprine (Kardachi and Newcomb, 1978).

Table 2.2 summarises this research. The studies should be interpreted with caution. Some involved small samples and were poorly controlled. In general they found no differences (Oshrain et al, 1983; Safkan and Knuuttila, 1984; Seymour et al, 1987) or detected less inflammatory change with prednisolone or azathioprine use (Schuller et al, 1973; Kardachi and Newcomb, 1978; Oshrain et al, 1979; Been and Engel, 1982; Sutton and Smales, 1983; Tollefsen and Johansen, 1985; Markitziu et al, 1990). This has been taken to indicate that lymphocytes do not exert protective effects (Genco and Slots, 1984).

In the last 15 years human immunodeficency virus (HIV) infection has emerged as a new disease in which lymphocytes are predominantly affected. Research of people with this disease may elucidate the role of lymphocytes in the immunoregulation of periodontitis. 
Table 2.2. Studies of the periodontal health of people taking immunosuppressive drugs.

\begin{tabular}{|c|c|c|}
\hline Study & Type of study & Findings \\
\hline Schuller et al. (1973) & $\begin{array}{l}\text { Uncontrolled cross-sectional. } \\
33 \text { people taking azathioprine } \\
\text { for renal transplant }\end{array}$ & $\begin{array}{l}\text { No correlation between } \\
\text { 'periodontal disease', plaque \& } \\
\text { age. Implied inhibited immune } \\
\text { response \& inflammation }\end{array}$ \\
\hline Kardachi \& Newcomb (1978) & $\begin{array}{l}\text { Cross-sectional, } 20 \text { patients } \\
\text { taking azathioprine \& } \\
\text { prednisolone cf. } 20 \text { healthy } \\
\text { subjects }\end{array}$ & $\begin{array}{l}\text { Gingivitis less common \& less } \\
\text { severe in study group }\end{array}$ \\
\hline Oshrain et al. (1979) & $\begin{array}{l}\text { Cross-sectional, } 20 \text { patients } \\
\text { taking azathioprine } \& \\
\text { prednisolone for renal } \\
\text { transplant, } 20 \text { dialysis patients } \\
\& 20 \text { healthy subjects }\end{array}$ & $\begin{array}{l}\text { No differences between groups } \\
\text { in clinical parameters. Plaque } \\
\text { not associated with gingivitis or } \\
\text { attachment loss in study group }\end{array}$ \\
\hline Been \& Engel (1982) & $\begin{array}{l}9 \text { month longitudinal study. } \\
4 \text { people taking azathioprine \& } \\
\text { prednisolone cf. } 9 \text { controls }\end{array}$ & Reduced BOP in study group. \\
\hline Oshrain et al. (1983) & $\begin{array}{l}\text { Longitudinal over } 2 \text { to } 4.5 \text { yrs } \\
6 \text { patients taking prednisolone } \\
\& \text { azathioprine cf. } 6 \text { controls }\end{array}$ & $\begin{array}{l}\text { No differences except mean } \\
\text { Gingival Index increased in } \\
\text { study group. }\end{array}$ \\
\hline Sutton \& Smales (1983) & $\begin{array}{l}\text { Cross-sectional, } 102 \text { people } \\
\text { immunosuppressed (many } \\
\text { azathioprine \& prednisolone) cf. } \\
111 \text { matched controls. }\end{array}$ & $\begin{array}{l}\text { Less pocketing, more recession } \\
\& \text { BOP in study group. } \\
\text { Similar destructive periodontal } \\
\text { disease. }\end{array}$ \\
\hline Safkan \& Knuutila (1984) & $\begin{array}{l}\text { Cross-sectional, } 27 \text { people } \\
\text { taking prednisolone cf. } 26 \\
\text { matched controls }\end{array}$ & $\begin{array}{l}\text { No differences in gingivitis, } \\
\text { probing depths or recession. }\end{array}$ \\
\hline Tollefsen \& Johansen (1985) & $\begin{array}{l}\text { Cross-sectional, } 26 \text { people } \\
\text { taking Azathioprine/ } \\
\text { cyclophosphamide \& } \\
\text { prednisolone cf. haemodialysed, } \\
\text { uraemic or healthy controls. }\end{array}$ & $\begin{array}{l}\text { Less gingival bleeding in } \\
\text { immunosuppressed group. } \\
\text { Other parameters similar. }\end{array}$ \\
\hline Seymour et al. (1987) & $\begin{array}{l}\text { Longitudinal. } 24 \text { people taking } \\
\text { Azathioprine or cyclosporin. } \\
\text { Baseline } 7 \text { - } 10 \text { days after renal } \\
\text { transplant. } 3 \text { \& } 6 \text { month review }\end{array}$ & $\begin{array}{l}\text { Gingival inflammation similar. } \\
\text { Gingival hyperplasia with } \\
\text { cyclosporin. }\end{array}$ \\
\hline Markitziu et al. (1990) & $\begin{array}{l}\text { Longitudinal, } 6 \text { month review } \\
19 \text { people taking prednisolone }\end{array}$ & $\begin{array}{l}\text { Less BOP, shallower pockets, } \\
\text { increased recession } \& \text { unaltered } \\
\text { alveolar bone height }\end{array}$ \\
\hline
\end{tabular}

cf. = compared with.

$\mathrm{BOP}=$ bleeding on probing

Complete references in text. 


\subsubsection{Lifestyle.}

Lifestyle could confound the relationship between HIV infection and periodontal health. This section reviews available evidence implicating recreational drug use (particularly tobacco and alcohol) and stress as risk factors for periodontal diseases.

\subsubsection{1 $\quad \underline{\text { Tobacco }}$}

Palmer (1987) reviewed tobacco and oral health and concluded that: $i$, tobacco use was associated with less gingival inflammation; $\mathrm{ii}$, most of the periodontal destruction in smokers was plaque associated but smoking may exert a small independent effect; iii, ANUG was more common in smokers; iv, the limitations of periodontal research prevented the elucidation of the extent to which tobacco acted independently, confounded or mediated the effect of plaque.

Subsequent research has clarified the picture. 28 year follow-up of 165 people first examined in 1959 found tobacco smoking to be a significant predictor of periodontal attachment loss, independent of the effect of plaque (Ismail et al, 1990). The relative risk for chronic periodontal disease is 2.5 times greater among smokers (Bergstrom, 1989), the effect is radiographically demonstrable even among participants with good oral hygiene (Bergstrom et al, 1991) and surgical and non-surgical periodontal treatment is less effective in smokers (Preber and Bergstrom, 1985; Preber and Bergstrom, 1990). More recently, a large cross-sectional study found smoking to be associated with both increased recession and pocketing and identified a possible dose-response relationship (Martínez-Canut $e t a l$, 1995). In a cross-sectional study in which age, sex, plaque and frequency of 5 possible periodontopathogens were similar in study and control groups Stoltenberg et al. (1993) found an odds ratio of 5.3 for a mean probing depth of $3.5 \mathrm{~mm}$ or more in smokers compared to non-smokers. Similarly, Haber et al. (1993) attributed a risk to smoking of $51 \%$ for the presence of periodontitis in 19-30 year olds and 32\% in 31-40 year olds in a study where plaque scores were similar in smokers and non-smokers. The authors considered the association between smoking and periodontal status so strong that periodontitis studies should be controlled for tobacco use.

Similar periodontopathogens are present in both smokers and non-smokers (Preber et al, 1992) and the effect of tobacco may be mediated via systemic alteration of the immune system by reducing polymorphonuclear leukocyte adhesion and phagocytosis (MacFarlane et al, 1992) and local cytotoxicity (Cuff et al, 1989). 


\subsubsection{4. $\quad$ Alcohol}

Kranzler et al. (1990) identified relationships between indicators of alcohol use and periodontal disease. There were no associations between alcohol use and oral hygiene although the authors accepted that their data wereunlikely to identify this. Sakki et al. (1995) investigated the presence of periodontal pockets in relation to a range of socio-economic, demographic, dental behaviour and lifestyle variables in a study of 700 Finnish people. Pocketing was strongly associated with alcohol consumption but there was considerable confounding between dental health behaviour and lifestyle and it was "improbable that the main effect was biological".

\subsection{Other recreational drugs}

Use of recreational drugs has been shown to be high in some populations of homosexual men. However, a literature search on Ovid Medline for text words of abstracts identified no publications which linked gingivitis or periodontal diseases with narcotics, amphetamines, marijuana smoking, cocaine or amyl nitrite. A small uncontrolled study of drug users found poor oral hygiene, especially among heroin users (Silverstein, 1973).

\subsubsection{4 $\quad \underline{\text { Stress }}$}

Few would doubt that being diagnosed with HIV infection can be stressful or that the diagnosis might affect the everyday life of even an asymptomatic individual.

Stress has been associated with an increased incidence of ANUG among soldiers on difficult postings, students during exam terms and other negative life events (Pindborg, 1951; Roth, 1951; Giddon et al, 1963; Cohen-Cole et al, 1983).

Stress is an imbalance in the interaction between an individual's perception of demands placed on him/her and perceived capability to meet those demands (Cox, 1978). To overcome the difficulties of measuring this interaction, urinary endogenous steroid (17 hydrocorticosteroids and cortisol) concentrations have been used as markers and are raised among people with ANUG (Maupin and Bell, 1975; Cohen-Cole et al, 1983). Cogen et al. (1983) considered the role of steroids as mediators of stress in the aetiology of ANUG and suggested that elevated steroid levels might decrease PMN chemotaxis and phagocytosis (see section 2.2.1.1). Giddon et al. (1969) observed digital vasomotor hypotonicity, elevated finger pressures and slowed finger cooling in students with a history of 2 or more episodes of ANUG. The findings were thought to suggest a peripheral vasomotor defect in ANUG although no role for this in the aetiology of the disease was offered. 
The relationship between 'stress' and chronic periodontal diseases has been investigated for some time. Researchers have subjected laboratory animals to stressors (immobilisation, starvation, loud noises, bright lights, injection of irritants, cold, immersion in water and/or exercise) and made clinical, microscopic and physiological assessment of the periodontium (Ratcliff, 1956; Fedi, 1958; Gupta et al, 1960; Stahl, 1961; Shklar, 1966; Manhold et al, 1971). Some animals which survived had clinical changes (Ratcliff, 1956; Shklar, 1966) or fewer osteoblasts, more osteoclasts and coarser more irregular periodontal membrane on microscopy (Ratcliff, 1956; Fedi, 1958; Gupta et al, 1960; Stahl, 1961; Shklar, 1966). These studies used small samples, were often not quantitative and assessors were not blinded to the experimental/control status of the animals. The authors ignored the risk of injury to the rat's periodontium caused by trying to escape immobilisation or the toxic effects of injections.

Early human studies associated psychological features with periodontal diseases. Moulton et al. (1952) observed 'excessive dependence on parents and childish oral pleasures' among 32 patients attending a dental clinic with ANUG, periodontitis or gingivitis and Belting and Gupta (1960) reported more periodontal disease in psychiatric patients compared to other hospital patients even when controlled for frequency of brushing. Mean probing depths were increased in people from broken homes or with marital problems, greater somatisation or higher levels of hysteria and severe periodontal disease has been associated with greater recent stress (Baker et al, 1961; Green et al, 1986). No association was found between rate of bone loss and anxiety among 50 patients with periodontitis and students or psychiatric patients in a study by Miller et al. (1956). These studies have often been small and qualitative and have used outdated models of periodontal disease and stress. In many cases there is no direct link between psychological state and periodontal health and the confounding effects of oral hygiene and tobacco use are ignored.

Recent advances in psychoimmunology (Ballieux, 1991) suggest several ways in which the immune response may mediate the effect of stress on periodontal health but measurement problems make them difficult to confirm.

\subsubsection{5}

Age

Periodontitis often takes decades to become measurable. Accordingly, it is more common and severe with advanced years because age confounds disease duration (Abdellatif and 
Burt, 1987). Periodontitis is not a consequence of age (Papapanou and Lindhe, 1992), is associated with poor oral hygiene irrespective of age (Suomi et al, 1971) and does not progress in adults with good oral hygiene (Löe et al, 1978).

\subsubsection{Medications}

Medications can influence periodontal health as has been discussed in section 2.2.3.3. Antibiotics can be used to treat periodontitis and acute necrotising ulcerative gingivitis (Slots and Rams, 1990). Phenytoin, sodium valproate, cyclosporin and nifedipine may cause gingival overgrowth especially in patients with poor oral hygiene (Seymour et al, 1987; Hassell, 1990).

\subsection{Periodontal Diseases and HIV Infection}

Current models describe periodontal diseases as the interaction between bacterial pathogens and the host's immune system. It follows that HIV-related changes in the immune system may alter that interaction. Whilst several oral manifestations of HIV may affect the periodontium (including cytomegalovirus infection, lymphomata, Kaposi's sarcoma and Mycobacterium avium complex (Vallejo et al, 1989; Glick et al, 1991; Moskow and Hernandez, 1991; Chapple et al, 1992; Dodd et al, 1993; Palmer et al, 1993; Shiboski and Winkler, 1993; Robinson et al, 1996) the remainder of this review will limit itself to diseases unique to the periodontium.

Abundant references, often poorly substantiated, have established periodontal diseases as part of the range of HIV-associated conditions. (Farthing, 1986; Greenspan et al, 1986; Saadoun, 1987; Farthing et al, 1988; Pindborg, 1989; EEC-Clearinghouse, 1991). Original reports of the disease comprise a small number of cases and few describe the methods used to diagnose the conditions (Dennison et al, 1985; Pindborg et al, 1986; Pindborg and Holmstrupp, 1987; Abel and Andriolo, 1989; Rosenstein et al, 1989). Larger studies have produced conflicting reports on the characteristics, epidemiology, microbiology and natural history of the diseases.

In the following sections a range of changes first described only 10 years ago are reviewed (Dennison et al, 1985). The field is developing rapidly and a consensus is yet to emerge. Some disagreement stems from methodological issues but much confusion is a result of the evolution of knowledge. For example, some conditions have been given up to 4 names. Where possible, the nomenclature agreed at the $1992 \mathrm{EC}$ consensus conference will be used (EC-Clearinghouse, 1993). This nomenclature is particularly useful since it does not include 
references to HIV-infection and allows the changes to be more readily recognised among people without HIV. Opinion is divided as to whether the changes are specific to HIVdisease (Smith et al, 1993; Garfunkel and Glick, 1995). Because the diagnoses may not be interchangeable, reports before 1992 are presented using their original terms and diagnoses.

\title{
2.3.1 Periodontal Diseases seen in HIV infection
}

Periodontal diseases described in HIV-infection can be placed in four broad categories

\author{
$\mathrm{i}$, Unusual forms of gingivitis \\ ii, Localised destructive disease \\ iii, Gross destructive disease \\ iv, Accelerated conventional periodontitis.
}

\subsubsection{Unusual forms of gingivitis.}

In 1986 workers at the Oral AIDS Centre of the University of California at San Francisco (UCSF) described two periodontal lesions, a generalised atypical gingivitis (ATYP) and an ANUG-like lesion (Winkler et al, 1986). The gingivitis was characterised by bright erythema, often extending to the alveolar mucosa, with oedema, and usually affecting all the teeth but not responding to improved oral hygiene. They subsequently renamed this condition HIV-Gingivitis (HIV-G) (Winkler et al, 1988) and described three different presentations; 1 . an intense red linear border at the gingival margin, 2. petechiae like red patches affecting the attached and/or unattached gingiva, 3. a generalised erythema affecting the attached and/or unattached gingiva. Figures 2.2 and 2.3 depict distinct red gingival margins in a 13 year old haemophiliac boy with AIDS and punctate patches on the attached gingiva of a homosexual man with HIV. Figure 2.4 shows ulceration of the gingival margin and diffuse erythema of the attached gingiva in a homosexual man with HIV infection.

This group of diseases was discussed at a consensus conference in 1992 and renamed Linear Gingival Erythema (LGE) (EC-Clearinghouse, 1993). The presumptive diagnostic criterion for this condition became 'a distinct fiery red band along the margin of the gingiva. The amount of erythema is disproportionately intense for the amount of plaque seen. No ulceration is present and there is no evidence of pocketing present'. The conference noted that the microbiology of the lesion was undefined so no definitive criterion could be made. However, LGE was said not to respond to the removal of dental plaque and calculus. 
Figure 2.2. Distinct red gingival bands in a 13 year old haemophiliac boy with AIDS.

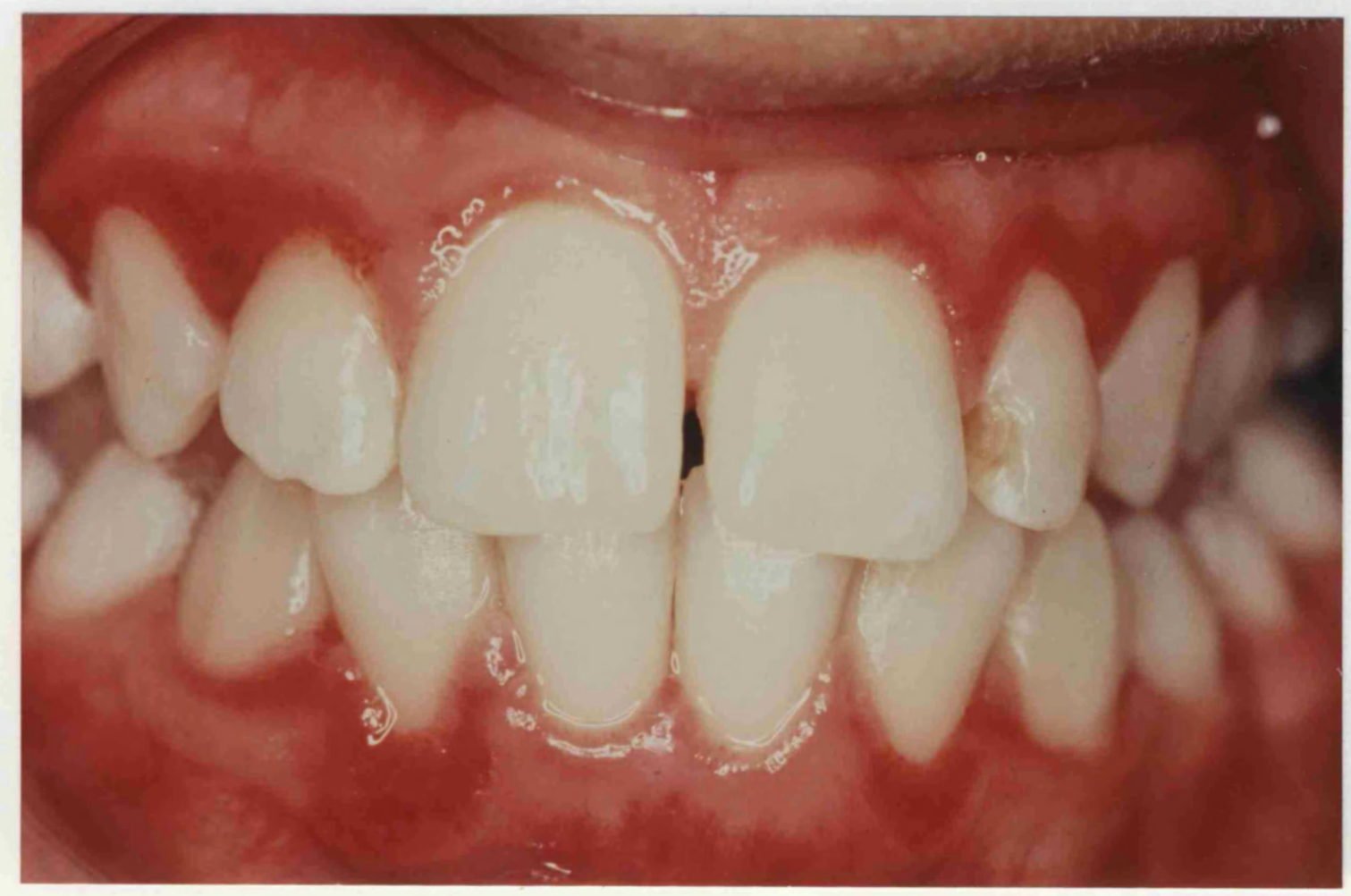

Photograph courtesy of Guy Palmer, Department of Special Needs Dentistry, UMDS. 
Figure 2.3. Punctate erythema of the attached gingiva of a homosexual man with HIV.

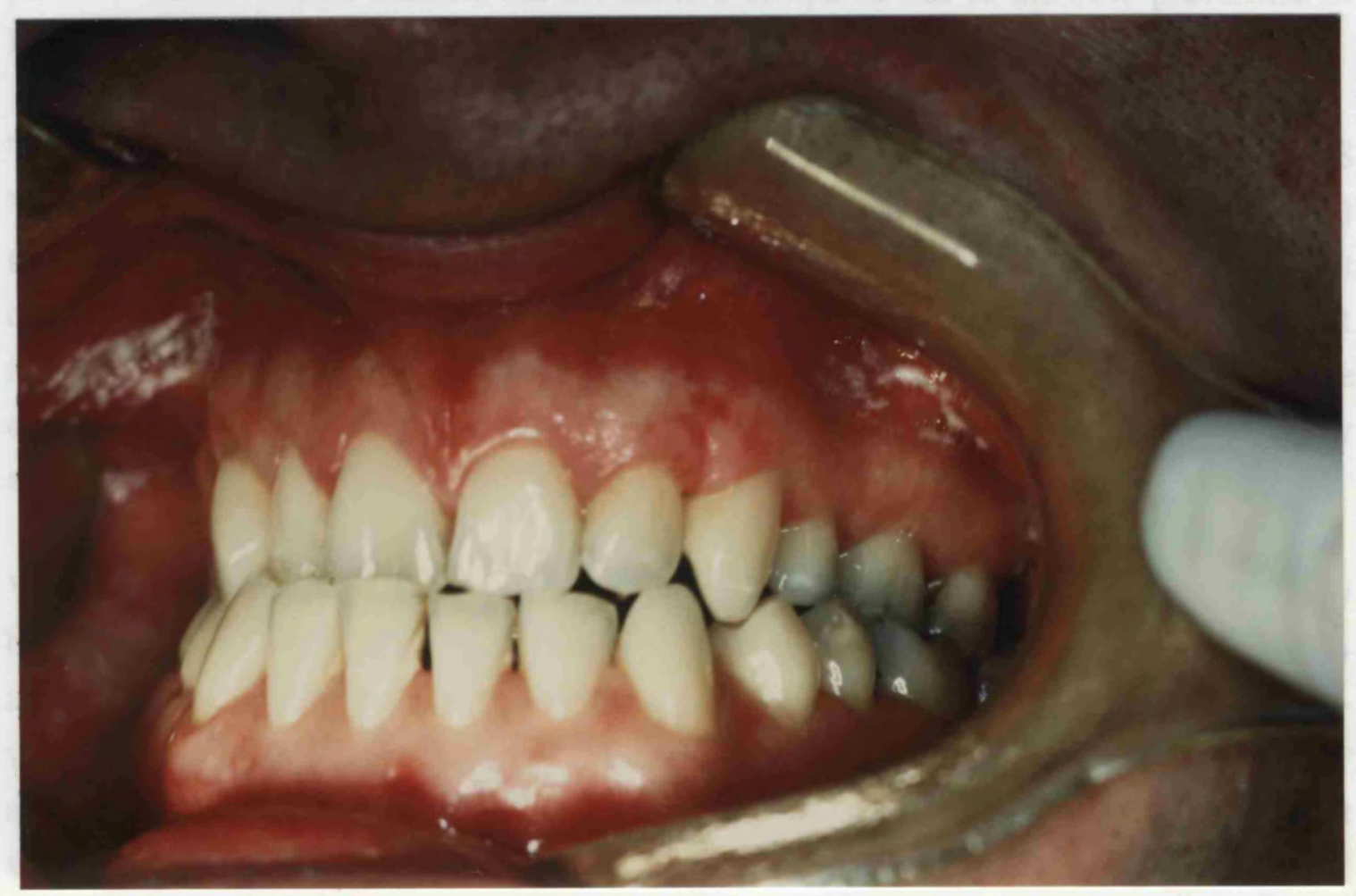




\subsubsection{2 $\quad$ Localised destructive disease}

In 1985, Dennison et al. (1985) described acute necrotising ulcerative gingivitis in a group of homosexual men not tested for HIV. HIV-associated ANUG has since been reported by Pindborg et al. (1986) and Pindborg and Holmstrup (1987) and the disease included in lists of the oral manifestations of HIV infection (Greenspan et al, 1986; Pindborg, 1989). A similar disease (HIV-necrotising gingivitis) was associated with HIV by the EECClearinghouse in 1991 (EEC-Clearinghouse, 1991). More recently, Soubry et al. (1995) have reported necrotising periodontal disease in $2.2 \%$ of 50880 attenders at an oral surgery clinic in Kigali, Rwanda. 224 of these patients were HIV tested of whom 182 (81.3\%) were positive. There were no controls but the authors compared this to the $30 \%$ prevalence of HIV in the local population.

The ANUG-like lesion described by Winkler et al. (1986) was originally named AIDS Virus Associated Periodontitis (AVAP) and subsequently HIV-Periodontitis (HIV-P) (Winkler et al, 1988). They characterised this disease by severe pain, marked localised soft tissue necrosis, ulceration and interproximal cratering (Figure 2.4). Lesions were not associated with deep pocket formation but a loss of crestal bone coincident with soft tissue destruction was : - described. In some cases bone was exposed and subsequently sequestrated (Winkler et al, 1988).

Winkler \& Murray (1987) distinguished HIV-P from ANUG by its rapid progression. They believed ANUG could only cause bone loss after a number of years compared to $90 \%$ attachment loss documented over a six month period in one of their patients. Murray and coworkers (1988) investigated the subgingival plaque of a small number of sites with HIV-P and found the levels of spirochaetes present in the lesions to be variable and not consistent with the microflora associated with ANUG (see microbiology below).

A subsequent EC consensus conference renamed HIV-necrotising gingivitis Necrotising (ulcerative) gingivitis (NUG) (EC-Clearinghouse, 1993). The presumptive diagnostic criterion was 'Destruction of one or more interdental papillae. In the acute stage of the process ulceration, necrosis and sloughing may be seen, with ready haemorrhage and characteristic foetor'.

HIV-periodontitis was renamed Necrotising (ulcerative) periodontitis (NUP) (ECClearinghouse, 1993). This was defined as 'Periodontitis characterised by soft tissue loss as a result of ulceration or necrosis. Exposure, destruction or sequestration of bone may be seen, and the teeth may become loosened. Pain may be a prominent feature'. The conference 
Figure 2.4. Ulceration localised to the gingival margin and diffuse erythema of the attached gingiva in a homosexual man with HIV infection.

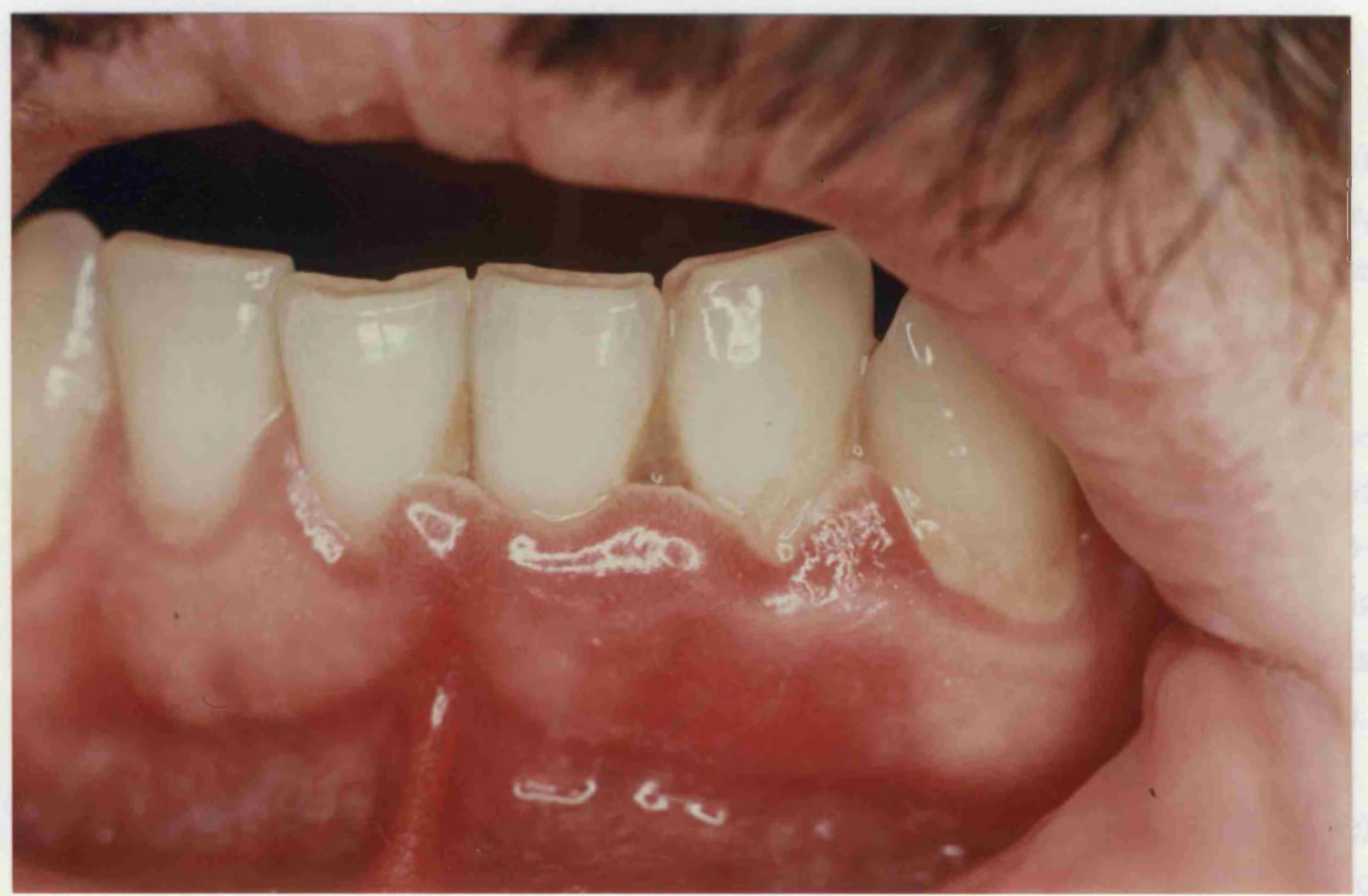


noted that destruction might extend over the muco-gingival junction and that the disease was chronic and could be seen with ulceration during an active phase or without ulceration during a less active phase.

No definitive criteria were available to support the clinical diagnoses NUG and NUP.

It is apparent that the definition of NUG does not distinguish it from ANUG. In addition, both definitions aggregate acute ulcerative disease with their legacy of absent tissue. There is no published evidence to suggest that NUP is chronic and evidence of relapse was reported as recently as 1994 (Robinson and Zakrzewska, 1994).

\subsubsection{Gross destructive disease}

There are reports of people with AIDS with 'gangrenous stomatitis' (cancrum oris or noma) (Akula et al, 1989; Giovanni et al, 1989). Cancrum Oris is an extension of ANUG (Emslie, 1963), and Winkler et al. (1989) suggested that the lesions may originate in the periodontium (Figure 2.5).

There have also been reports of HIV-P complicated by destruction of alveolar bone (SanGiancomo et al, 1990; Williams et al, 1990; Felix et al, 1991). SanGiancomo and colleagues (1990) implied this was an extension of HIV-P, whereas Williams and coworkers (1990) described it as a complication. Neither group differentiated their observations from Cancrum oris. Smith et al. (1991) noted similar changes in 10 patients and reported a case with bone loss sufficient to cause an oro-antral fistula (Felix et al, 1991).

The EC-Clearinghouse (1993) grouped this range of diseases as necrotising (ulcerative) stomatitis and assigned the presumptive criterion 'Localised acute, painful ulceronecrotic lesion of the oral mucosa that exposes underlying bone or penetrates or extends onto contiguous tissues. These lesions may extend from areas of necrotising periodontitis'. No definitive criteria could be given. Histological features were said to be those of non-specific ulceration and the group stated that microbiological studies failed to identify a specific aetiological agent. There is no published data to support these statements and there is no threshold to discriminate between NUP and necrotising stomatitis. 
Figure 2.5. Extensive ulceration and necrosis in a 47 year old homosexual man with AIDS.

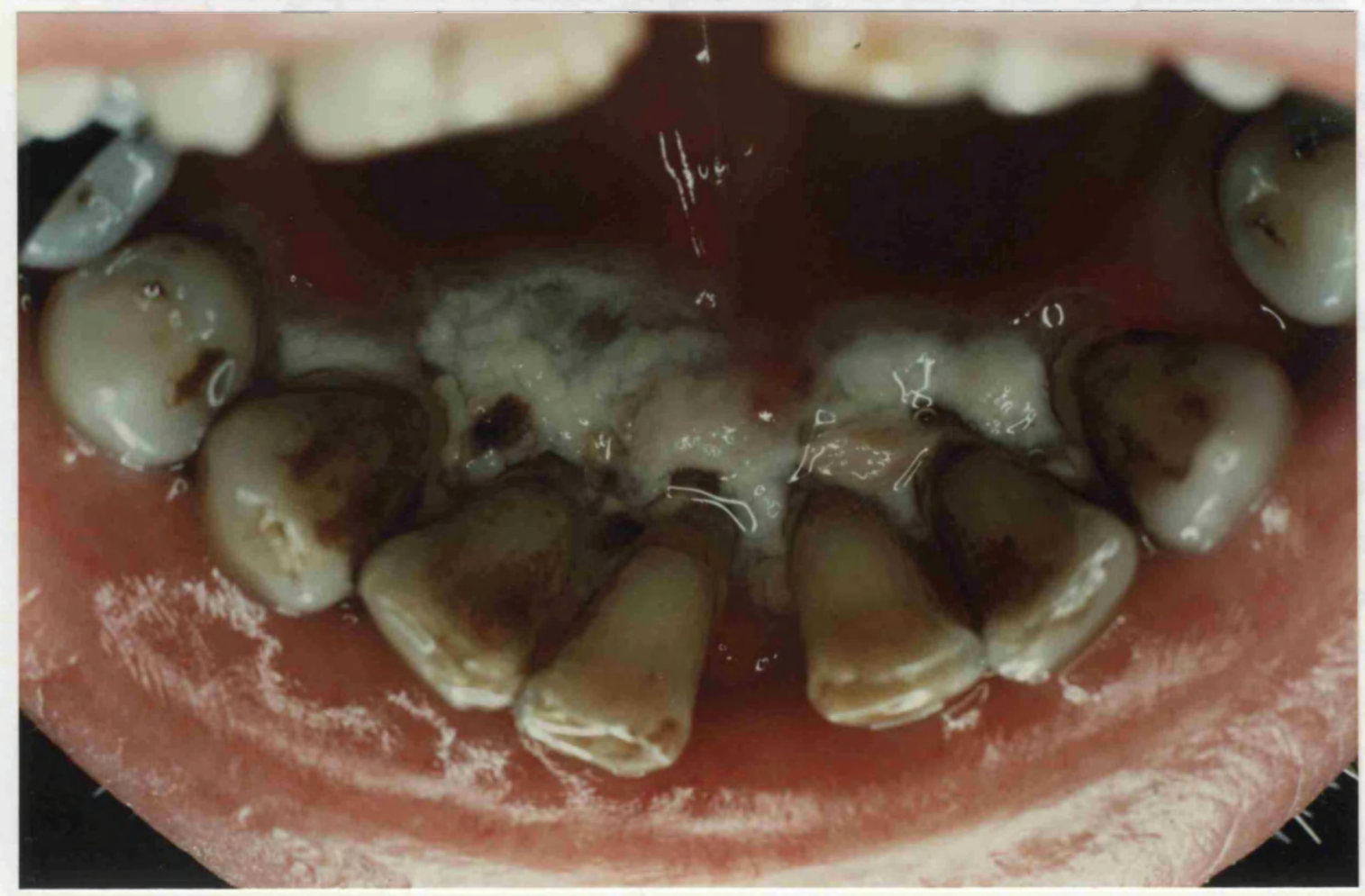




\subsubsection{Rapidly progressive periodontitis}

Pekovic et al. (1987) and Gornitsky and Pekovic (1988) were the first to describe rapid periodontal pocket formation in people with HIV and AIDS. Rosenstein et al. (1989) subsequently noted horizontal rapid bone loss with moderate inflammation in a case report.

Epstein et al. (1993) described periodontal pockets in 7 men with HIV and presented several clinical photographs. Some of these patients may have had adult periodontitis manifest by pocketing. However, all the pockets were interdental and 4 of the patients had gingival ulceration diagnosed by the authors, others may have had ulceration before presentation. One of the photographs shows buccal gingiva being retracted with air pressure from a syringe. This would be possible if there was no inter-dental soft tissue (which may have been destroyed by ulceration in some of these cases) whilst the buccal gingiva remained leaving a deep crater. Viewed from the buccal aspect this may have appeared like a pocket, even though there was no inter=dental soft tissue.

Levine and Glick (1991) entitled a review article Rapidly Progressive Periodontitis as an important clinical marker for HIV disease. The term 'rapidly progressive periodontitis' was used to describe the rapid tissue loss of HIV-associated periodontitis and not the specific clinical condition of that name (Page et al, 1983). However, Smith et al. (1995) cite this paper as further evidence of RPP in HIV infection, and the same group included RPP in their own classification of HIV-associated periodontal disease but cited no evidence to support the inclusion (Smith et al, 1993).

\subsubsection{Prevalence and incidence of periodontal diseases seen in HIV infection}

\subsubsection{1 $\quad$ Cross-sectional studies}

The epidemiology of HIV-related periodontal changes remains confused. For example, Silverman et al. (1986) reported 'periodontal disease' in 17\% of 375 homosexual men with or at risk for AIDS (Silverman et al, 1986). Careful reading of the paper suggests that AIDS (and so HIV infection) was only confirmed in $40 \%$ of the sample.

Numerous reports suggest that HIV-associated periodontal changes are common. Researchers at UCSF reviewed more than 400 patients with HIV-G and HIV-P over 6 years and published quantitative data for 113 of these (Winkler et al, 1988). However, no data on the denominator population were presented and the figures must be considered in the context of the prevalence of HIV infection in San Francisco. At the time of these reports the 
cumulative total of AIDS cases was 4000 and the prevalence of HIV infection would have been much greater.

Studies with an epidemiological approach are reviewed in Table 2.3. The table presents the first author, year and size of papers identified on Ovid Medline or papers they cited. Much research has been carried out on patients attending dental clinics so that selection bias may have over-represented the prevalence of periodontal diseases. Other studies have been poorly controlled, whilst others have not used well defined criteria to diagnose periodontal changes. The studies are reviewed in detail below.

Gornitsky et al. (1987) observed signs of adult periodontitis including 'redness, oedema, bleeding to pressure at the gingival margin and pockets' in 11 of 21 dental patients with AIDS of whom six had severe bone resorption. In a similar study of 181 people with AIDS attending a dedicated dental service, 24 patients had severe gingival ulceration and $55 \%$ had 4-5 mm pockets (Klein et al, 1991). 92\% of the patients were injecting drug users and since the study was uncontrolled these findings may reflect high levels of background disease in this population. Murray and colleagues (1991) also reported a high prevalence of HIV-G (49\%) and HIV-P (22\%) among a group of injecting drug users who had sought dental treatment at a special clinic.

Similarly, Zakarian et al. (1988) retrospectively reviewed 150 patients with AIDS or ARC treated at a dedicated dental clinic in New York. Of these, $12 \%$ had 'interproximal necrosis resembling ANUG' and 5\% had 'rapidly progressive periodontitis'. In addition to the points raised above, these data relate to patients' experience of disease over several years rather than point prevalence.

In a review of 178 patients with HIV referred to an oral physician, Laskaris et al. (1992) reported necrotising gingivitis in $10 \%$ of patients, HIV-Gingivitis in 5\%, HIV-Periodontitis in $18.5 \%$ and osteomyelitis in $0.6 \%$. Inevitably there was selection bias but the diagnostic criteria for the diseases were explicit. The criterion for HIV-periodontitis was 'severe inflammation, spontaneous bleeding, pain, soft tissue necrosis, pocketing (sic), rapid and severe bone destruction'. The authors discussed the likelihood of chronic adult periodontitis being diagnosed as HIV-periodontitis using this criterion. 
Table 2.3. Prevalence studies of periodontal health in HIV infection.

\begin{tabular}{|c|c|c|c|c|c|c|c|}
\hline Author & $\begin{array}{l}\text { Study } \\
\text { group } \\
\text { (n) }\end{array}$ & $\begin{array}{l}\text { Controls } \\
\text { (n) }\end{array}$ & $\begin{array}{l}\text { Selection } \\
\text { bias }\end{array}$ & $\begin{array}{c}\text { Diagnostic } \\
\text { criteria }\end{array}$ & $\begin{array}{l}\text { Prevalence } \\
\text { LGE } \\
\text { (or similar) } \\
(\%)\end{array}$ & $\begin{array}{l}\text { Prevalence } \\
\text { ulceration } \\
(\%)\end{array}$ & Notes \\
\hline $\begin{array}{l}\text { Phelan et al. } \\
\text { (1987) }\end{array}$ & 103 & 0 & $\mathrm{~N}$ & $\mathbf{N}$ & & & No severe PD \\
\hline $\begin{array}{l}\text { Reichart et al. } \\
\text { (1987) }\end{array}$ & 110 & 0 & $\mathrm{Y}$ & & 3.6 & 5.5 & $10.9 \%$ RPP \\
\hline $\begin{array}{l}\text { Gornitsky et al. } \\
\text { (1987) }\end{array}$ & 21 & 20 & Y & & & & $55 \% \mathrm{PD}$ \\
\hline $\begin{array}{l}\text { Zakarian et al. } \\
\text { (1988) }\end{array}$ & 150 & 0 & Y & $\mathbf{N}$ & & 12 & 5\% RPP \\
\hline $\begin{array}{l}\text { Schulten } \text { et al. } \\
\text { (1989) }\end{array}$ & 70 & 0 & $\mathrm{~N}$ & $\mathrm{~N}$ & & & $13 \% \mathrm{PD}$ \\
\hline $\begin{array}{l}\text { Melnick et al. } \\
\text { (1989) }\end{array}$ & 141 & 606 & $\mathrm{~N}$ & $Y$ & & 4.3 & $5 \% \mathrm{PD}$ \\
\hline $\begin{array}{l}\text { Tukutuku et al. } \\
\text { (1990) }\end{array}$ & 83 & 0 & $\mathrm{~N}$ & $\mathrm{~N}$ & & & $66 \%$ gingivitis \\
\hline $\begin{array}{l}\text { Swango et al. } \\
\text { (1991) }\end{array}$ & 230 & 0 & $\mathrm{~N}$ & $\mathrm{~N}$ & & 25 & $49 \%$ Red band \\
\hline $\begin{array}{l}\text { Apaiza et al. } \\
\text { (1991) }\end{array}$ & 15 & 0 & $\mathrm{Y}$ & $\mathrm{N}$ & 0 & 0 & \\
\hline $\begin{array}{l}\text { Friedman et al. } \\
\text { (1991) }\end{array}$ & 63 & 0 & $\mathrm{~N}$ & $\mathrm{~N}$ & & (3.2) & Disease normal \\
\hline $\begin{array}{l}\text { Klein et al. } \\
\text { (1991) }\end{array}$ & 181 & 0 & Y & $\mathrm{N}$ & "Common" & 13.2 & $55 \% \mathrm{PD}$ \\
\hline $\begin{array}{l}\text { Murray et al. } \\
\text { (1991) }\end{array}$ & 51 & 0 & $\mathrm{Y}$ & $\mathbf{N}$ & 49 & 22 & \\
\hline $\begin{array}{l}\text { Laskaris et al. } \\
\text { (1992) }\end{array}$ & 178 & $\mathrm{~N}$ & $\mathrm{Y}$ & Ambiguous & 5 & 10.1 & \\
\hline $\begin{array}{l}\text { Thompson et al. } \\
\text { (1992) }\end{array}$ & 390 & $\mathbf{N}$ & $\mathrm{N}$ & $\mathbf{Y}$ & "Many" & 7.2 & \\
\hline $\begin{array}{l}\text { Winkler et al. } \\
\text { (1992) }\end{array}$ & 18 & 27 & $\mathrm{~N}$ & & 0 & 0 & \\
\hline $\begin{array}{l}\text { Riley et al. } \\
\text { (1992) }\end{array}$ & 200 & 0 & $\mathrm{~N}$ & $\mathrm{Y}$ & 10.5 & 1 & \\
\hline $\begin{array}{l}\text { Masouredis. et of } \\
(1992)\end{array}$ & 136 & 0 & $\mathrm{~N}$ & YY & $39 / 50$ & $4 / 6$ & $\begin{array}{l}2 \text { sets of } \\
\text { criteria }\end{array}$ \\
\hline $\begin{array}{l}\text { Grbic et al. } \\
\text { (1995) }\end{array}$ & 121 & 83 & $\mathrm{~N}$ & $Y$ & $<38.5$ & $<5.2$ & Controls similar \\
\hline $\begin{array}{l}\text { Smith et al. } \\
\text { (1995) }\end{array}$ & 29 & 27 & $Y$ & $\mathrm{Y}$ & 0 & 6.9 & AL in HIV+ve \\
\hline
\end{tabular}


Tukutuku et al. (1990) diagnosed 'moderate' or 'severe' gingivitis in $55(66 \%)$ of 83 hospitalised patients with AIDS in Zaire. The authors noted an increased prevalence of gingivitis in this sample compared with that in Nigeria and Zaire in two studies carried out fifteen and eleven years earlier (Henshaw and Adenjubi, 1975; Ducka-Karsa, 1979). These comparisons are questionable.

Even among samples selected from those seeking care, other workers have found little or no evidence of unusual periodontal diseases in people with HIV infection. In a small study of 15 people with HIV infection attending a hospital in Mexico, only 2 cases corresponding to descriptions of HIV-G were identified. No cases of HIV-P were found (Apaiza et al, 1991).

\section{$\underline{\text { Reduced selection bias }}$}

Unusual periodontal changes are less common among samples not selected for oral disease. No cases of 'severe periodontal disease' were noted among a consecutive sample of 103 people (28\% drug users and $72 \%$ homosexual- or bisexual men) with AIDS not selfselected for oral manifestations of HIV infection (Phelan et al, 1987). Only 1 case of gingival bleeding was reported in this study.

Schulten et al. (1990) noted periodontal disease in 9 of 70 patients (13\%)(34 with AIDS and 36 with HIV infection) attending an internal medicine department. No mention was made of the nature or severity of the disease or of the diagnostic criteria used.

Friedman et al. (1991) examined 63 men (41 homosexual, 15 IJUs) with HIV attending an infectious disease clinic and detected neither differences in gingival index, probing depths or attachment loss between men with early HIV disease or AIDS, nor between their sample and similar parameters in the general population. No unusual gingivitis was detected and only 2 subjects had AIDS-associated periodontitis. US military personnel with HIV were screened by Swango et al. (1991). One quarter had necrotic destruction of interdental papillae and $49 \%$ had an erythematous band along the gingival margin but the authors have been at pains to point out this was not HIV-gingivitis as described by Winkler et al (1987, 1988, 1989) (P. Swango. Personal communication).

Riley at al. (1992) reduced selection bias by recruiting 200 homosexual men attending HIV studies at the US National Cancer Institute. Using strictly defined diagnostic criteria 21 (10.5\%) of their sample had HIV-associated gingivitis ('intensely red free gingival margins, pockets $\leq 3 \mathrm{~mm}$ '), $2(1 \%)$ had necrotising ulcerative periodontitis ('similar to ANUG but with loss of attachment and bone'). None had ANUG or HIV-periodontitis although the criterion 
for the latter was very similar to the one for NUP ('rapid onset and progression of destruction of periodontal attachment, pain, spontaneous bleeding, lack of deep pockets and simultaneous loss of alveolar crest bone and gingival margin').

A study by Masouredis et al. (1992) involving 136 homosexual men with HIV attending a San Francisco AIDS clinic used two sets diagnostic criteria for HIV-associated periodontal diseases. The first set was the more stringent:

HIV-G (must satisfy 1 and 2; plus either 3 or 4 )

1 Bleeding on probing

2 Gingival index greater than 1 in at least one site

3 Presence of punctate or diffuse gingival erythema of the attached gingiva

$4 \quad$ Red band of inflammation of the marginal gingiva

HIV- P (Must satisfy 1 and 2 plus at least two of the remaining 4 characteristics)

1 Presence of HIV-G in at least one site

2 Attachment loss to pocket depth ratio of greater than 1.5 in any one site

3 Patient complaint of bone pain

$4 \quad$ Presence of exposed bone

$5 \quad$ Presence of soft tissue or osseous necrosis

6 Cratered papilla

In the second set item two was removed from both definitions so that probes were not required.

These definitions were the most mutually exclusive and unambiguous used at the time. Two points of note remain. Firstly, in the Gingival Index of Löe and Silness (1963) (from which the definition for HIV-G is derived. J. Winkler, personal communication), bleeding on probing scores '2' thus the second criterion for HIV-G is superfluous. Secondly, it will be seen that neither definition of HIV-P required the presence of ulceration. The prevalence of HIV-G using the two definitions was $31 \%$ and $50 \%$ and HIV-P was seen in $4 \%$ and $6 \%$ of subjects.

Thompson et al. (1992) reported NUG in $7.2 \%$ of 390 soldiers with HIV of unknown transmission category. No data relating to gingivitis was presented but 'marginal gingivitis characteristic of HIV-associated gingivitis' was present in 'many' of the participants. 


\section{Controlled studies}

In a controlled, blinded study of 803 homosexual men (141 with HIV infection) attending a counselling centre in Seattle (Melnick et al, 1989) severe periodontal disease was diagnosed by the presence of 'extensive gingival recession with marked gingival inflammation, clinically evident alveolar bone loss and extrusion of pus from gingival pockets'. Seven (5\%) of the HIV positive subjects had severe disease but the prevalence of periodontal disease in the control group was extremely low, with only 1 case in 606 men (mean age 34.4 years). Based on these findings, the authors concluded that periodontal disease had a significant association with HIV infection but was not 'highly common'.

Winkler et al. (1992) conducted a controlled, blinded study of eighteen homosexual or bisexual men with asymptomatic HIV infection and found no cases of HIV-G or HIV-P nor any significant difference in their periodontal health. It is possible that the sample was too small to detect what may be an uncommon range of diseases.

Smith et al. (1995) compared 29 patients with HIV referred to a dental hospital by genitourinary medicine clinics and dentists with 27 other hospital patients. The patients with HIV were homosexual men (20), heterosexuals (5) and injecting drug users (4) and the control group comprised 2 known HIV negative homosexual men and 25 heterosexuals assumed to be HIV negative. There were similar numbers of teeth and similar proportions of sites with plaque, bleeding on probing and redness in both groups but a greater proportion of sites had suppuration in the patients with HIV.

Mean attachment loss tended to be higher $(p=0.099)$ and there were more sites with $3 \mathrm{~mm}$ or more of lost attachment in the patients with HIV (18.2\% vs. $7.3 \%)$. The attachment loss tended to be around the lower incisors. The use of means and the site as the unit for analysis reduces the usefulness of these data (see section 3.5.2.1). However, the patients were also dichotomised as 'widespread' or 'localised' attachment loss by whether they had attachment loss at more or less than $30 \%$ of sites. Nine patients with HIV and 2 in the control group had the more extensive pattern (31.0\% vs. $7.4 \%, p=0.059$, chi sq.). Participants with HIV and extensive attachment loss had more sites with redness and with suppuration and higher mean attachment loss (sic) than those with localised attachment loss, regardless of HIV status. Again the lower incisors were most commonly affected.

At the time of examination 1 patient with HIV had ANUG and another had 'suspected' ANUG. One had had HIV-P progressing to oro-antral fistula in the past. Informatively, the authors summarise this in the following words: 'the incidence of these diseases ... was restricted to 3 patients at baseline examination'. 
Lamster et al. (1995) reviewed the cross-sectional studies of periodontal health in HIV infection in a similar style to this and included two other papers. The first was the study by Silverman et al. (1986) in which not all the participants had HIV infection. In the second, a series of 20 patients attending a dental clinic with 'rapidly progressive periodontitis' who were at 'high risk for AIDS' were investigated for HIV infection. Sixteen were found to be positive (Tenenbaum et al, 1991). The authors concluded that periodontal disease may be a first clinical presentation of HIV infection and that further research was needed to confirm its predictive value. Both of these studies were omitted above where the aim was to review quasi prevalence studies.

The research recently reported by Grbic et al. (1995) is considered in the discussion in chapter 5.

\subsubsection{2 $\quad$ Longitudinal Studies}

Cross-sectional studies are useful for describing the prevalence of acute conditions but are less so for chronic and irreversible diseases. Longitudinal studies are the gold standard for the study of periodontal attachment loss. Five longitudinal studies are summarised in Table 2.4 .

Table 2.4. Longitudinal studies in people with HIV infection not receiving specialist periodontal treatment.

\begin{tabular}{|l|c|c|c|l|}
\hline Authors & $\begin{array}{c}\text { Study } \\
\text { group(n) }\end{array}$ & $\begin{array}{c}\text { Controls } \\
(\mathrm{n})\end{array}$ & $\begin{array}{c}\text { Follow up } \\
(\mathrm{m})\end{array}$ & \multicolumn{1}{|c|}{ Findings } \\
\hline $\begin{array}{l}\text { Cornick and } \\
\text { Robinson. 1991 }\end{array}$ & 57 & 8 & 24 & $\begin{array}{l}\text { Small but significant loss attachment unrelated to } \\
\text { disease status }\end{array}$ \\
\hline Drinkard et al. 1991 & 106 & 0 & 11 & $\begin{array}{l}\text { No HIV-associated disease } \\
\text { No differences by disease status }\end{array}$ \\
\hline Barr et al. 1992 & 102 & 33 & 20 & $\begin{array}{l}\text { More attachment loss with disease status \& age. } \\
\text { Average GI greater in HIV infection } \\
\text { 5 cases NUG, 6 cases red bands, no HIV-P }\end{array}$ \\
\hline Yeung et al. 1993 & 30 & 10 & 18 & $\begin{array}{l}\text { Mean PDI increased in HIV among those with AL } \\
\text { at incept. } \\
\text { 6 cases of ANUG }\end{array}$ \\
\hline Smith et al. 1995 & 29 & 0 & 9 & No attachment loss \\
\hline
\end{tabular}


Cornick and Robinson (1991) followed a group of homosexual men with HIV infection for three years. Initially the sample consisted of 57 men with known HIV infection, 8 known not to be infected and 28 of indeterminate status. After three years only 25 men with HIV remained in the study. No significant differences in gingival indices, probing depths, attachment levels or crestal bone height were associated with HIV infection or severity of HIV disease.

The study by Drinkard at al. (1991) had an incept cohort of 106 participants: 100 homosexual or bisexual men and 1 woman, of whom 97 had asymptomatic HIV infection and 9 had AIDS-related-complex (ARC). The cohort had diminished to 25 by 48 week follow-up. Plaque index, gingival index, bleeding index and probing depth were compared for the 6 index teeth specified by Ramfjord (1967) in each participant. Mean plaque index, gingival index, bleeding index and probing depth did not differ between those with the asymptomatic and ARC groups at any of the 4 visits. There was a tendency for decreases over time in the plaque, gingival and bleeding indices among the asymptomatic participants and small increases over time in the plaque and probing depths of those with ARC. There were no incidents of cratering or necrosis in either group.

This study did not record attachment loss in the absence of pocketing (a feature allegedly characteristic of HIV-associated disease) and the use of means may have resulted in the loss of subtle changes by swamping. However, the authors concluded that this sample was and remained periodontally healthy and attributed this in part to concern to maintain oral health among the participants. The participants were recruited via an AIDS clinical trial unit and $69 \%$ had visited the dentist in the preceding year.

All 135 subjects in the study by Barr et al. (1992) were homosexual or bisexual men and were followed up for 20 months by which time the cohort had diminished to 114 . The proportion of sites with $3 \mathrm{~mm}$ or more attachment loss was greater in the men with HIV ( $0.5 \%$ vs. $0.0 \%, p=0.04$, Wilcoxon 2 sample rank sum test). In the men with HIV there was confounding between immunosuppression and age. By stratifying for age $( \pm 35 y)$ this effect was reduced and the relationship between immunosuppression (CD4 lymphocyte count \pm 200 cells $/ \mathrm{mm}^{3}$ ) persisted, most prominently in older men. Men with lower CD4 counts were 6.16 times more likely to lose attachment $(95 \% \mathrm{CI}=1.95-19.40)$. Men who had attachment loss during the study had more gingival inflammation at baseline. These data remain the most convincing evidence implicating HIV in periodontal disease. Sadly, the researchers did not separate pocketing and recession to allow description of the nature of the attachment loss (Marta Lopez, personal communication). 
The same group compared mean gingival indices and linear regressions of gingival index against plaque index in the groups with and without HIV. Mean Gingival Index increased over time with greater regression coefficients in men with HIV $(p=0.04)$ which the authors took to indicate greater sensitivity to plaque. However, the gingival index is an ordinal scale not suited to averaging or regression analysis (Löe and Silness, 1963). The $P$ value and the analytic method render these findings less compelling than those relating to attachment loss.

The incidence of HIV-associated periodontal diseases was also recorded in this sample. During the 20 months. Five cases of NUG and 6 of erythematous banding were seen suggesting an annual incidence of approximately $3.5 \%$ and $4.7 \%$ respectively. There was no apparent relationship between either change and CD4 count, although the number of cases available to analyse this were tiny.

Yeung et al. (1993) measured Plaque Indices, Gingival Indices and Periodontal Disease Indices (PDI) on the Ramfjord (1967) teeth in 30 men with asymptomatic HIV infection enrolled in a trial for the anti-retroviral drug Zidovudine. Participants were examined every 6 months for 18 months. There were no differences between the drug and placebo groups so the data were aggregated and compared with a control group of 10 healthy men matched for age and plaque index. The study and control groups had similar plaque and gingival indices but mean PDI increased more in men with HIV ( $p=0.005, \mathrm{t}$ test). The attachment loss in the study group was in men with periodontal disease at baseline. There were six cases of ANUG during the study period, suggesting an annual incidence of approximately $13.3 \%$. There was considerable swamping in this study because of the use of the PDI. In particular it does not distinguish between pocketing and recession (Ramfjord, 1967). Despite the loss of sensitivity inherent in their methods, a relationship was detected between loss of periodontal attachment and HIV infection.

The most recent longitudinal study was by Smith et al. (1995) who reported follow up on 29 people with HIV examined at baseline and 3, 6 and 9 months later. Mean probing depths and the proportion of sites with bleeding were reduced at 3 months despite an increase in the proportion of surfaces with plaque. The number of sites with a measured loss of attachment of $3 \mathrm{~mm}$ or more over the study period was less than the anticipated false positive rate. This potentially useful study was marred by lack of follow up of the control group and by over reliance on the individual site as the unit of inference. 


\subsubsection{Conclusions}

The disparity in the findings of these studies at least partly results from selection bias, lack of controls, lack of blinding, use of participants who acquired HIV through different routes and the study of subjects receiving preventive periodontal care. In some cases subjects were not examined by dentists. When diagnoses were assigned to participants there were often no universally applied diagnostic criteria. In some cases criteria were ill-defined, ambiguous or unstated. There has not often been systematic use of standard measures and indices of periodontal health. Some of the measures used were not sensitive to the changes anticipated in HIV infection.

Winkler et al. (1989) suggested that geographical differences may explain the variation in the findings of different researchers. They also suggested that the UCSF groups studied originally had very low levels of disease which allowed detection of subtle changes in periodontal health. The comparison of health in homosexual men and drug users lends some support to the latter argument (Grbic et al, 1995), but the scarcity of data prevents firm conclusions being drawn.

The prevalence and incidence of unusual forms of gingivitis vary between 0 and $50 \%$ in the studies above and this seems largely attributable to the diagnostic criteria used. Masouredis et al. (1992) demonstrated a difference of $20 \%$ with different criteria. With the exception of this study, larger controlled studies of samples not selected for oral disease detect a prevalence of HIV-associated gingivitis/LGE of less than $10 \%$.

The destructive diseases are also less common in better designed studies and have a prevalence of less than $5 \%$. The incidence is between 0 and $13 \%$. Only one study had an incidence greater than $3.5 \%$

It is difficult to distinguish the 'rapidly progressive periodontitis' associated with HIV from other presentations of attachment loss. Several references to this term in the literature do not relate to the clinical entity known by that name. Also, the nature of lost attachment is rarely presented in reports of periodontal health among people with HIV. However, the case reports suggest that measures and indices which detect conventional periodontal diseases are required in this area of research. 


\subsubsection{Pathogenesis of Periodontal Diseases seen in HIV infection}

Both plaque and host factors have been investigated in the pathogenesis of this range of diseases. Inevitably, most research into host factors has concentrated on immune changes associated with HIV disease.

\subsubsection{1 $\quad$ Plaque}

Three approaches have been used to investigate the microbiology of periodontal changes seen in HIV infection: i, cross-sectional investigations relating the microflora to periodontal health and disease; ii, investigations at predominantly healthy sites and iii, prospective studies to detect microflora which predicted disease. Within these epidemiological approaches there are two basic methods. Techniques such as immunofluorescence and DNA probes detect specific putative pathogens and are relatively quick and inexpensive. Culture methods with selective media will detect all cultivable microflora. This technique does not rely on assumptions of pathogenicity but is time consuming and expensive.

The use of antimicrobial drugs in the management of HIV disease creates difficulty investigating the microbiology of HIV-associated periodontal diseases. Despite this a range of techniques have been used and a relatively consistent picture is emerging.

\subsection{Microflora associated with health and disease}

The UCSF group used culture techniques, indirect immunofluorescence (Murray et al, 1988; Murray $e t a l, 1989$ ), indirect ELISA (Murray $e t a l, 1987$; Grieve $e t a l, 1988)$ and DNA probes (Murray et al, 1991) to investigate the microflora of both HIV-G and HIV-P. The group did not try to identify large numbers of the organisms but found the flora present in both lesions was comparable to that of classical periodontitis. In particular Porphyromonas gingivalis (formerly Bacteroides gingivalis), Prevotella intermedia (formerly $B$. intermedius,) Fusobacterium nucleatum, Actinobacillus actinomycetemcomitans, Eikenella corrodens and Campylobacter rectus(formerly W. recta) were identified. Murray et al. (1988) stressed that the levels of spirochaetes present in the lesions were variable and not consistent with the greater numbers of spirochaetes seen in ANUG but were circumspect the following year when they wrote 'More extensive studies would be required to determine whether the microbiota of HIV-associated periodontitis is similar to or different from that seen in ANUG' (Murray et al, 1989). 
Zambon et al. (1990) cultivated the predominant subgingival microflora from 11 patients with AIDS and moderate periodontitis. Immunofluorescence microscopy was used to determine the presence of putative periodontal pathogens in 50 subjects (6 with a history of ANUG, 7 with diagnosed HIV-gingivitis and 1 with diagnosed HIV-periodontitis). Fortythree bacterial species were identified including many of the Gram-negative anaerobes associated with conventional periodontitis. These findings are compatible with those of Murray et al. (1989) although A. actinomycetemcomitans was detected in small numbers, and only by immunofluorescence. The authors account for this discrepancy by pointing out that only one patient in their sample exhibited HIV-periodontitis.

In a study involving 14 subjects, Rams et al. (1991) compared healthy sites with sites diagnosed with HIV-periodontitis. The predominant cultivable microflora of the HIV-P were Gram negative anaerobes including $C$. rectus, $P$. intermedia and $A$. actinomycetemcomitans and the Gram positive anaerobe Peptostreptococcus micros. The authors also likened this pattern to that of conventional periodontitis in normal subjects.

Moore et al. (1993) used both culture techniques and DNA probes to compare the species diversity and amounts of micro-organisms in marginal gingivitis or adult periodontitis in people with and without HIV. Considerable effort was made to standardise for periodontal health and exclude injecting drug users or people who had recently taken antimicrobials. There were statistically significant differences in the organisms present in HIV positive and negative participants with periodontitis but this was less than might be expected by chance. Increased Mycoplasma salivarium was also noted in people with HIV but the authors concluded there were 'few if any biologically significant differences in the floras of HIV+/AIDS subjects and non HIV subjects'.

In a companion report to the longitudinal clinical study by Smith et al. (1995), Cross et al. (1995) used DNA probes and a colony lift method to measure the prevalence and amounts of 7 species of subgingival microflora (A. actinomycetemcomitans, $P$. intermedia, $P$. gingivalis, B. forsythus, Capnocytophaga ochracea, Veillonella parvula and C. rectus). Participants with HIV had a higher mean count of $P$ gingivalis, largely attributable to the sub-group with widespread attachment loss. There were no differences between those with localised attachment loss regardless of HIV status.

Zambon et al. (1990) cultivated Clostridium difficile, Clostridium clostridiiforme, Enterococcus faecalis, Enterococcus avium and Klebsiella pneumoniae from the subgingival plaque of people with AIDS. These organisms are not widespread in the plaque of people without AIDS. Likewise, 12 of the 14 subjects studied by Rams et al. (1991) harboured a 
range of unusual organisms including Enterobacteria spp, Pseudomonas aeruginosa, $C$. albicans and Klebsiella spp.

Murray et al. (1988) noted that HIV-G and HIV-P sites harboured significantly more Candida albicans than control sites in the same subjects and in an uncontrolled study Zambon et al. (1990) detected yeasts in the plaque of 31 of 50 patients with AIDS. This carriage rate is much higher than in adults with periodontitis (Slots et al, 1988). Candida spp. were detected in the plaque of 8 of 28 people with HIV investigated by Moore et al. (1993). They suggested this 'low' prevalence was because they had excluded drug users. However, differences in periodontal health and HIV disease progression may also have distinguished their participants from those of earlier researchers. Candidal hyphae and pseudohyphae have been found in 7 of 27 gingival biopsies from people with HIV but not in 16 HIV negative control samples (Odden et al, 1994). Candidal invasion was significantly more frequent in people who had had necrotising periodontal disease and the authors suggested that the Candida spp. might contribute to the development of the disease.

A new oral spirochaete has also been detected in people with HIV (Rosenstein et al, 1993). Monoclonal antibodies were used to identify this organism in 21 people with HIV infection. It was found in 2 of 10 people with 'healthy' gingiva compared to 10 of 11 who had disease akin to adult periodontitis. The authors were planning further investigation of the role of this organism.

\subsection{Healthy sites in people with HIV.}

Lucht et al. (1990) detected no significant differences between the microflora of the saliva or gingival crevices of 30 people with HIV infection (10 with no clinical signs, 10 with ARC and 10 with AIDS) compared to a control group. The subjects in this study had all undergone dental treatment before enrolment in the study and were considered to have a 'healthy dental status' which may account for differences between these findings and those above.

This group have also recorded the subgingival microflora, numbers of lymphocyte subsets and periodontal indices for a very similar group of 40 people (Lucht et al, 1991). No association was found between periodontal health and amounts of visible plaque or counts of $A$. actinomycetemcomitans, Capnocytophaga spp. or $P$. gingivalis. 


\subsection{Prospective study}

The prospective component of the study by Cross $e t$ al. (1995) failed to identify a microorganism or amount of an organism which predicted attachment loss. This was partly attributed to the small number of sites which had attachment loss. $P$ gingivalis was present in increasing numbers and $V$. parvula in decreasing numbers in late HIV disease. Although this was compatible with pathogenic and protective features, the authors pointed out that these organisms were so frequent that they were unlikely to be useful predictors of attachment loss.

\subsubsection{Host factors}

Not surprisingly, the host factor most often explored in relation to HIV-associated periodontal changes is the degree of immunodeficiency. The indicators of immunodeficiency used have been clinical (history of opportunistic infections) or laboratory measures (local and systemic $\mathrm{T}$ lymphocyte counts).

This section reviews the research of host factors in HIV-associated periodontal changes by considering epidemiological research of non-ulcerative and ulcerative diseases and by a review of laboratory based research.

Many of the epidemiological studies discussed earlier have investigated periodontal changes in relation to the immunodeficiency or progression of HIV disease. The methodological flaws of research on the presence of unusual periodontal conditions (see above) are a greater barrier to investigation of this more precise problem and no consensus has been reached.

\subsection{Non-ulcerative diseases}

\subsection{Cross-sectional studies}

Three cross-sectional studies (total of 80 participants with HIV) report worse periodontal health with greater immunosuppression. In a small study in which 11 of 21 patients with HIV showed signs of adult periodontitis, 'periodontal disease' was more common with clinically advanced AIDS (Gornitsky and Pekovic, 1987). The 21 patients were divided between 5 clinical stages and so the numbers do not justify firm conclusions. Lucht $e t$ al. (1991) compared the gingival bleeding index, pocket depth and the number of sites with pockets equal to or greater than $4 \mathrm{~mm}$ in people with asymptomatic HIV infection, ARC, AIDS and HIV negative controls ( 10 in each group). All three measures were increased 
with immunodeficiency measured both by clinical stage and lower CD4 lymphocyte counts. Both these reports are unusual in that they describe increased pocketing in HIV infection (attachment loss and recession were not recorded). Finally, cross-sectional data from 29 people with HIV detected greater mean attachment loss and more extensive suppuration in later clinical stages of HIV disease (Smith et al, 1995).

Two cross-sectional papers (total of 263 participants) have failed to detect differences in periodontal health with degrees of immunodeficiency. One study of 63 men with HIV detected no differences in gingival index, probing depths or attachment loss in early HIV disease or AIDS (Friedman et al, 1991). Nor w iere there any differences in the prevalence of strictly defined periodontal conditions in the 200 men investigated by Riley et al. (1992).

\subsection{Longitudinal studies}

Even if the prevalence studies showed a consistent relationship between immunodeficiency and periodontal health, the findings would be inconclusive. HIV disease is chronic and it is difficult to distinguish the effects of immunodeficiency and disease duration in crosssectional studies. Somebody with advanced HIV disease might have periodontal changes not because they were severely immunodepressed but because they had been immunodepressed for a long time.

Three longitudinal studies have attempted to measure the rate of periodontal change for different degrees of immunodeficiency. Neither Cornick and Robinson (1991) (25 subjects at 2 year follow up) nor Drinkard et al. (1991) (25 at 48 week follow up) detected any differences in rate of change of standard periodontal measures between participants with different clinical stages of HIV at incept. By contrast, Barr et al. (1992) found a relative attachment loss of $3 \mathrm{~mm}$ or more was 6.16 times more common $(95 \% \mathrm{CI}=1.95-19.40)$ in men with CD4 counts of less than 200 cells $/ \mathrm{mm}^{3}$ in men of 35 years or more. The greater sensitivity to plaque with HIV reported by this group did not appear to be related to CD4 counts (See section 2.3.2.2 on problems with this analysis).

\subsection{Necrotising diseases}

Cross-sectional studies may be more useful for studying necrotising diseases since it is reasonable to assume that the onset of an acute disease is related to the current immune status rather than the deterioration over a number of years. Three such studies have investigated necrotising diseases and immune status as measured by CD4 counts. In a study of $230 \mathrm{HIV}$-infected US military personnel, necrotic destruction manifest by 'visible 
ulceration or cratering' (ulceration was present in all cases. P. Swango, personal communication) was more extensive at CD4 counts greater than $400 \mathrm{cells} / \mathrm{mm}^{3}$ and in smokers ( $p=0.06$ and $p<0.03$. ANOVA) (Swango et al, 1991). The prevalence of destruction was very high in this sample $(25 \%)$ which suggests there was some misdiagnosis.

Two studies related necrotising disease to lower CD4 counts. Thompson et al. (1992) found NUG to be more common in military personnel with CD4 counts less than 400 cells $/ \mathrm{mm}^{3}$ (data not given. $p<0.01$ ).

Glick et al. (1994) found 40 of their 44 patients with NUP had CD4 counts less than 200 (OR 20.8, 95\% CI $=4.9-87.1$ ). Follow-up data were reported including a life table analysis. $27 \%$ of patients with NUP died within 12 months but no control group data were reported to evaluate the additional risk of death conferred by the disease. Smoking and use of co-trimoxazole were compared between the 40 patients with NUP and AIDS and 35 'randomly selected' patients with AIDS but not NUP. There was no significant difference in tobacco use between the groups ( $31.8 \%$ Vs. $51.4 \%$ ) but fewer of those with NUP used cotrimoxazole $(9.1 \%$ Vs. $65.7 \%)$.

\subsection{Laboratory studies}

The only report of histological examination of Linear Gingival Erythema (formerly HIVGingivitis) was by Glick et al. (1990) who described the condition in one man with AIDS. The man had spontaneous gingival bleeding, erythematous bands along the gingival margins and petechiae of the attached gingiva. A biopsy sample was very vascular with no inflammatory cellular infiltrate. The gingival changes had been present for some time and the authors expected to find a lymphocyte, plasma cell and macrophage infiltrate of chronic gingivitis. The authors suggested that this was an incomplete inflammatory response caused by decreased cytokine production due to lymphocyte depletion and dysfunction of AIDS.

Immunohistochemical findings of the immune cells in the gingiva of people with HIV are compared in Table 2.5. T lymphocytes were absent from the gingiva in 7 of the 8 subjects investigated by Steidley et al. (1992) but present in decreased numbers compared to matched HIV negative controls in the other two studies (Gomez et al, 1995; Odden et al, 1995). This contrast is difficult to explain. Subjects in the study by Steidley et al. (1992) had higher peripheral CD4 counts than those in the other two studies (peripheral CD4 counts were not given by Gomez et al. but all 11 subjects had AIDS and the count is likely to be less than $200 \mathrm{cells} / \mathrm{mm}^{3}$ ). Nor do differences in periodontal health readily explain the 
discrepancy. Steidley et al. did not present details of the periodontal health of their subjects other than to say they had 'periodontal disease'. Subjects in the study by Odden et al. (1995) had varying periodontal health ranging from gingivitis to periodontitis (mean PAL $4.9 \mathrm{~mm}$ at the biopsy sites). Biopsies in the Gomez study were retrieved from files on the basis of having LGE. Since no diagnostic criteria were presented and data on clinical parameters were not available it may be that some of these subjects had conventional gingivitis. Odden et al. accounted for the difference as 'methodological differences'.

In the same paper, Odden et al. (1995) compared CD4/CD8 ratios in the gingiva and peripheral blood lymphocytes (PBLs) within each subject. Although the gingival lymphocyte counts reflected the lymphocytopenia of HIV disease, CD4/CD8 ratios were increased in gingival samples from subjects with HIV compared to controls $(p<0.05$, Wilcoxon matched pairs test). The authors suggested there was active recruitment of CD4 lymphocytes to the gingiva and cited similar findings from oral and gut mucosa. This group detected fewer CD4 cells in the connective tissue of 8 subjects with a history of HIVassociated necrotising periodontal disease compared to HIV-infected subjects without such a history (medians 10.5 Vs 20.5. $p<0.01$, Mann-Whitney test (MWWT)).

Gomez et al. (1995) also found fewer macrophages and higher proportions of neutrophils and plasma cells in their subjects with HIV.

Table 2.5. Immunohistochemical studies of immune cells in the gingiva of people with HIV.

\begin{tabular}{|c|c|c|c|c|c|}
\hline Study & $\begin{array}{l}\text { HIV+ve } \\
\text { (n) }\end{array}$ & $\begin{array}{c}\text { Mean serum } \\
\text { CD4 } \\
\left(\text { cells } / \mathrm{mm}^{3}\right)\end{array}$ & $\begin{array}{l}\text { Periodontal } \\
\text { health }\end{array}$ & $\begin{array}{l}\text { HIV-ve } \\
\text { (n) }\end{array}$ & Gingival T lymphocytes \\
\hline $\begin{array}{l}\text { Steidley et al } \\
\text { (1992) }\end{array}$ & 8 & 430 & $\begin{array}{l}\text { 'Periodontal } \\
\text { disease' }\end{array}$ & 6 & None in $7 / 8$ subjects \\
\hline $\begin{array}{l}\text { Gomez et al } \\
\text { (1995) }\end{array}$ & 11 & 'AIDS' & 'LGE' & 33 & Fewer in HIV \\
\hline $\begin{array}{l}\text { Odden et al } \\
\text { (1995) }\end{array}$ & 25 & 311 & $\begin{array}{l}\text { Gingivitis \& } \\
\text { Periodontitis }\end{array}$ & 13 & $\begin{array}{l}\text { Fewer in HIV than } \\
\text { controls }\end{array}$ \\
\hline
\end{tabular}

\subsubsection{Natural History of HIV-associated periodontal changes}

No clear evidence is available on links between the different HIV-associated periodontal changes. To Murray et al. (1988), the similar subgingival microflora seen in HIV-G and HIV-P suggested that HIV-G was an early stage of HIV-P with the flora remaining avirulent until advanced HIV infection allows disease progression. In one study of 113 
patients (42 with AIDS, 23 with ARC and 48 sero-positive men without ARC/AIDS), HIV-G was diagnosed in patients with normal CD4:CD8 ratios. HIV-P was found only in men with low CD4:CD8 ratios and more frequently in patients with OHL and candidiasis (Winkler $e t a l, 1988$ ). Baseline data on this group were described in 1988 (Winkler $e t a l$, 1988), but no follow up data have been reported. Grassi et al. (1990) monitored 12 patients with HIV-G for one year, none of whom developed HIV-P.

It is not possible to assess the extent to which attachment loss seen in HIV disease can be attributed to severe or repeated episodes of necrotising ulcerative disease or to a more conventional mode of periodontal attachment loss.

\subsubsection{Pathogenesis conclusions}

Microbiology of periodontal diseases is an imprecise science. There are no useful ways of identifying active sites of destruction and so it is difficult to associate specific organisms with disease. In addition, it is difficult to quantify the uncertainty of data collected in this type of microbiological research. The data are infrequently amenable to statistical analysis.

Two explanations for the microbiological role in the aetiology of HIV-associated periodontal diseases remain. The diseases may result from infection by the organisms associated with conventional periodontitis whose virulence increases with the immunédeficiency of HIV infection. Alternatively, the unusual organisms detected in people with HIV infection may contribute to the disease. Zambon et al. (1995) recently reviewed this question and concluded that the available studies 'support the concept that the HIV-associated periodontal diseases are due to the same types of predominantly anaerobic bacteria as are associated with periodontal disease in otherwise healthy subjects'. This seems to be the case but they added that 'closer examination of carefully defined subjects with forms of HIV-associated periodontal disease such as linear gingival erythema might demonstrate the role of opportunistic pathogens'.

There is some evidence implicating Candida spp. in necrotising ulcerative periodontal disease (Odden et al, 1994). This evidence is far from conclusive and does not exclude the flora usually associated with ANUG in the pathogenesis of necrotising disease. It may be that candidal infection is a confounder for immunosuppression.

The role of immunedeficiency is also unclear. A reasonable assumption is that the immunodeficiency per se causes periodontal changes and that this is related to the degree of immunodeficiency. However, cross-sectional studies of acute (ulcerative) diseases do not 
lend convincing support for this theory and it is difficult to separate the confounding effects of degree and duration of immunodeficiency.

\subsubsection{Management of Periodontal Diseases seen in HIV infection}

The UCSF team reported an evaluation of three management regimen for HIV-P based on a study of 30 patients (Grassi et al, 1988). All patients received oral hygiene instruction, scaling and root planing. Six patients were asked to irrigate their gingivae with povidoneiodine from a syringe 3 to 5 times daily for three months. Ten patients were asked to rinse with $15 \mathrm{ml}$ of $0.12 \%$ chlorhexidine solution twice daily for three months. Patients using the chlorhexidine rinses had lower mean probing depth (sic) at follow up although the validity of this measure is questionable. Winkler et al. (1989) subsequently recommended a more detailed regimen, based on their experiences at UCSF but not formally evaluated. This included:-

i. Debridement of the lesions to mechanically remove plaque, calculus and necrotic tissue. Frequent irrigation with Povidone Iodine during this phase may have a topical anaesthetic effect and help control bleeding (Grassi et al, 1988).

ii. Meticulous oral hygiene was stressed. Dentists or hygienists should instruct patients in the use of interproximal cleaning devices wherever tissue destruction has made oral hygiene difficult.

iii. Patients were prescribed $250 \mathrm{mg}$ Oral metronidazole four times a day for four or five days and $0.12 \%$ Chlorhexidine mouthwash twice daily. Antifungal therapy is prescribed if required.

iv. After the acute phase of treatment frequent follow up was recommended to monitor compliance with oral hygiene advice and for root planing to prevent relapse.

\subsection{Rationale for research.}

Although periodontal diseases have been established as a manifestation of HIV infection, the links between them remain unclear. HIV-related periodontal diseases undoubtedly exist and can be severe in some patients, yet relatively little is known about their prevalence, aetiology or progression.

Carefully constructed studies using appropriate sampling techniques and control populations are required to investigate the epidemiology of the disease and its natural history. Larger randomised controlled trials are also needed to test the efficacy of the treatment regimen for the diseases. 
The establishment of defining criteria would allow all forms of the disease to be recognised and enable the collection of precise prevalence data, comparison of results between research centres, and valid research of the pathogenesis of the diseases. This should demonstrate any geographical differences in the forms and distribution of the diseases and will be particularly necessary if the diseases are uncommon.

\subsubsection{Statement of aims of the research}

The specific aims of the research reported in this thesis were to:

i, establish whether there are periodontal changes associated with HIV infection

ii, assess the types prevalence and severity of periodontal changes in HIV infection

iii, identify possible aetiological factors of the periodontal changes associated with HIV

iv, develop and test diagnostic criteria for periodontal changes associated with HIV infection. 


\section{CHAPTER 3 METhodoLOGY}

This section discusses 4 strategies selected to meet the aims of this research. These were:

i) the development and reliability studies of a set of diagnostic criteria for periodontal changes associated with HIV infection

ii) a cross-sectional study to assess the prevalence, types and severity of periodontal changes seen in HIV infection and to identify possible aetiological factors for them among homosexual men attending a genito-urinary medicine clinic

iii) a case series to characterise gingival ulceration seen in HIV infection and a casecontrolled study of gingival ulceration to identify risk factors for the condition among patients with HIV attending a dedicated dental clinic

iv) Validation of diagnostic criteria for HIV-associated periodontal changes by comparing their predictive values for HIV infection in the study of the periodontal health of homosexual men with and without HIV.

The final part of this section considers methodological issues of epidemiology of periodontal diseases. The methods of each study are presented in the relevant chapters.

\subsection{Development and reliability of diagnostic criteria for periodontal changes associated with HIV (Chapter 4).}

Diagnostic tests are used to predict the presence of a disease. There are seven necessary features of any test, index or set of diagnostic criteria (Barnes et al, 1986; Grant et al, 1988; Greene, 1990):

i) simplicity relates to the ease, speed of use and the cost of the system

ii) objectivity requires explicit, unambiguous and mutually exclusive categories

iii) validity has two components: sensitivity (ability to detect the condition where it is present) and specificity (ability to not detect the condition where it is absent)

iv) reliability (reproducibility) has three components: freedom from inter-examiner and intra-examiner variation and from the effects of biological variability

v) acceptability to the subject by being quick, painless and not demeaning

vi) precision relates to the ability of an index to detect small changes

vii) amenability to statistical analysis. 
These features are inter-related and an accurate index is derived from the combination of the features. No index is wholly accurate since there are usually . trade offs between sensitivity and specificity, and between precision and reliability. Emphasis is placed on different characteristics depending on the context. In epidemiological research involving many subjects a simple presumptive criterion may be more useful than a' complex 'gold standard' criterion.

A diagnostic test must be reliable before it can be validated. Validation studies are futile if test results are not reproducible.

\subsection{A cross-sectional study of the periodontal health of homosexual men with HIV (Chapter 5).}

The intention was to conduct a controlled blinded study of the periodontal health of homosexual men with HIV infection to identify the prevalence. types and severity of periodontal diseases associated with the infection and to identify possible aetiological factors for any changes.

Cross-sectional studies study outcomes without prior knowledge of predictors (Newman et $a l, 1988)$. Data is collected on a single occasion to describe and provide prevalence data for outcome and predictor variables and identify associations between the two (choice of which to label 'predictors' and 'outcomes' depends on the research hypothesis). Large amounts of data can be collected and networks of links can be examined quickly to reveal crosssectional associations. Such studies cannot establish causal relationships and are impractical for rare outcomes. They are susceptible to prevalence/incidence or survivor bias in which effects of a risk factor for disease duration may appear to effect prevalence. Cross-sectional studies are suitable for studying exposures which cannot change such as hereditary factors but less useful for exposures such as smoking where current tobacco use may not be so pertinent as smoking history.

\subsubsection{Selection of populations}

A control group was required to provide a yardstick of the background disease in the population and blinding was used to reduce measurement bias (Sitthi-amorn and Poshyachinda, 1993). This study was restricted to homosexual men to remove any confounding effect by sex, sexual behaviour or unforeseen effects associated with injected drug use. Homosexual men were targeted since they constitute the vast majority of cases of 
HIV infection in southern England. Approximately 800 men were required for this study. A genito-urinary medicine clinic setting was selected with large accessible populations of homosexual men with and without HIV who might be similar in other respects and to provide access to detailed medical data. Use of a non-dental setting reduced selection bias.

\subsection{Gingival ulceration in HIV infection: a case series and case control study (Chapter 6).}

This study aimed to characterise the gingival ulceration seen in HIV infection and to identify possible risk factors for the disease.

Case series are cross-sectional studies in which samples are drawn from diseased populations. They are suited to describing diseases rather than identifying risk factors. Case series can be compared with 'unaffected individuals in case control studies in which exposure to possible predictors is considered retrospectively. Case control studies are suited to uncommon outcomes since they provide a great deal of information from few cases and are useful for hypothesis generation as they can examine many predictor variables (Schlesselman, 1982).

Case control studies have natural limitations. Only one outcome can be studied and its incidence or prevalence cannot be calculated. They are inefficient for rare exposures and are prone to sampling and retrospective measurement bias. Unrepresentative samples are likely if those who do not demand treatment, are undiagnosed or who recover are not selected (Newman et al, 1988). Selection of controls is particularly important. The central condition for case control studies is that controls are selected independently of exposure status (Rothman, 1986). This and other sources of bias can be reduced by sampling the study and control groups in the same way.

Measurement bias is an inherent weakness of case control studies because people with diseases are examined and remember differently than those without disease. This type of bias can be reduced by having standardised examination protocols, recording data before the outcome occurs and by blinding examiners and participants to case/control status and risk factors. 
Case control studies can be restricted to any type of case, for example, by severity of disease, sex of those affected etc. They can be used to study prevalent cases if the duration is not related to exposure. However, if the risk factor affects both the aetiology and duration of disease, a case control study based on prevalent cases will be unable to distinguish the two effects. Cases are better selected by incidence within a given period.

The control group provides an estimate of the exposure distribution in the source population. It does not have to be identical to the case group apart from the relevant exposure factors (it is difficult to determine which factors are relevant in advance). The important features of controls are: that they are subject to the same selection factors and their continuous eligibility to become cases. Each case in a case control study should be eligible as a control until the time of disease onset. Each control should be eligible to become a case later during the study period (Rothman, 1986).

\subsection{Validity of diagnostic criteria for periodontal changes associated with HIV (Chapter 7).}

Tests and criteria should be evaluated by how well they predict disease compared to a gold standard diagnosis (Browner et al, 1988; Altman, 1991). Validity is measured by the sensitivity, specificity and positive and negative predictive values (PPVs and NPVs) of the test.

Samples of adequate size are needed to measure the sensitivity, specificity, PPV and NPV accurately and the subjects in the study should closely represent the people on whom the test will be used. Diseased subjects should have mild or early rather than frank disease and a control group, similar to the study group in all other respects is required (Browner et al, 1988).

Both the test and the gold standard should be applied to all subjects in a standardised and blinded fashion. Where no gold standard is available another marker for disease can be used. For example, the predictive values of diagnostic criteria for HIV-associated periodontal diseases could be assessed using HIV negative populations as controls.

Predictive values are related to the prevalence of the disease in the population (Galen and Gambino, 1975; Browner et al, 1988; Altman, 1991). The sensitivity, specificity and 
predictive values of the test should be reported for different prior probabilities. This is less relevant if there is no gold standard and no estimate of the prevalence of the disease.

\subsubsection{Sensitivity, specificity and predictive values}

The sensitivity of a test is its ability to predict the disease where it is present. It is the proportion of subjects with the disease and a positive test. The specificity is the ability to predict the absence of the disease where it is absent and is the proportion of subjects without the disease who test negative.

Sensitivity and specificity do not measure the probability of a correct diagnosis. This is provided by the positive and negative predictive values. The PPV is the proportion of patients with a positive test result who are correctly diagnosed and the NPV the proportion of patients with a negative result correctly diagnosed.

Predictive values are dependent on the presence of disease. At lower prevalences it is less likely that a positive test denotes presence of disease, i.e. specificity decreases. Thus with rare diseases a more specific test is necessary.

\subsubsection{Limitations of diagnostic test studies}

Diagnostic tests studies are subject to random and systematic error (Browner et al, 1988).

Some people with disease will have normal results. To avoid this problem samples should be sufficiently large to determine confidence intervals for the specificity and sensitivity of the tests.

Random error also occurs if the investigator defines a positive result after the study, perhaps by using a summary measure of several tests as a predictor variable. There may be a chance combination of results which successfully predicts within the study population but which is not generalisable. Summary measures are best designed before data analysis. This implies a two stage design with a test phase to define the positive result and a validation phase conducted on another sample.

Sampling bias (study population does not represent the general population) may limit diagnostic test studies if subjects have severe disease with marked changes or if controls are healthy volunteers. To overcome this problem samples should be drawn from the population on which the test will be used. If the prevalence of the disease in the study 
population is higher than in the general population the predictive value will be overemphasised.

Measurement bias occurs if the outcome is known to the person conducting the test especially if there is scope for interpretation of borderline results. This type of bias can be reduced by blinding the examiner to the outcome and by using objective tests.

\subsection{Methodological issues in epidemiology of periodontal disease}

A fundamental requirement of epidemiology is that the type, extent and severity of disease must be recognised and classified in a way that allows analysis. Characteristics of periodontal diseases pose particular biological, statistical and measurement problems.

\subsubsection{Biological issues}

\subsubsection{The presence of disease}

Early models of periodontal disease considered disease to be ubiquitous, constant, chronic and inexorable. The frequency of periodontal disease has been questioned and Socransky $e t$ al. (1984) have proposed the burst theories in which periodontal destruction is episodic with quiescence or remission between bursts (Socransky et al, 1984). The statistical basis for these theories is now challenged (see below) but we no longer assume that all sites with attachment loss have active disease. Instead, attachment loss is regarded as the legacy of past disease (Griffiths et al, 1988; Burt, 1991) and the need for a marker of current disease activity is reflected in the amount of research to find one (Johnson, 1991). Even if a marker of destructive periodontal disease activity were available it would need to be validated for all types of destructive disease and particularly for an unusual population such as those with HIV.

Lack of disease markers entails two approaches. Cross-sectional studies can measure lost attachment as a proxy for current destruction, accept considerable misclassification (Mertens, 1993) and settle only for hypothesis generation in exchange for quicker and less expensive studies. Changes in attachment levels can be measured longitudinally to aid inference of cause and effect. In addition to the inherent problems of cohort studies, very few sites change, the degree of change is small and follow up must be delayed until there is sufficient change to distinguish from measurement error. Since a small minority of adults experience significant periodontal attachment loss in a lifetime (Miller et al, 1987), studies of attachment level change need to be conducted for at least 2 years (Cowell et al, 1975). 
Measurement error of attachment loss creates sufficient false positives (sites incorrectly thought to have lost attachment) to seriously hamper longitudinal research (Imrey, 1986; Ralls and Cohen, 1986; Gunsolley and Best, 1988).

\subsubsection{Multifactorial nature of periodontal diseases}

The multifactorial nature of periodontal diseases requires that the many factors must be accounted for in research. Extraneous factors may confound, mediate or interact with a relationship between an exposure and effect (Rothman, 1986; Davey-Smith and Phillips, 1992; Datta, 1993).

Control of confounding is essential. Confounders must be identified then restricted or matched and/or considered in stratified or multivariate analysis (Newman et al, 1988). Control of confounding either makes selection of subjects more rigorous or makes samples larger.

\subsubsection{3 $\quad$ Random/biological variability}

Variation between subjects which cannot be accounted for is 'random variation'. Its effect is to reduce epidemiological precision and again this demands larger samples (Rothman, 1986).

\subsubsection{Additional HIV-associated problems}

Specific problems of diagnosing HIV-associated changes are superimposed on these uncertainties. Not all the periodontal changes in people with HIV can be attributed to HIVassociated disease but must be distinguished from this background of conventional diseases which people with HIV may have or have had before becoming infected.

A further obstacle is the lack of comprehensive and objective documentation of HIVassociated changes, hitherto characterised only by clinical impressions. Since clinical diagnosis can be subjective and since there are properties common to both HIV-associated changes and conventional diseases (gingival erythema, attachment loss, gingival ulceration, pain) there is danger of misclassification. Periodontal research of people with HIV has only rarely used explicit diagnostic criteria for the conditions.

To a lesser extent research is also hampered by the names given to this range of periodontal changes. The terms HIV-Gingivitis and HIV-Periodontitis used since 1988 imply that the 
conditions are exclusive to people with the infection (Winkler et al, 1988) This may be misleading as Garfunkel and Glick (1995) have suggested. The names make the conditions difficult to diagnose in people without HIV. For these reasons an international consensus on new names without the HIV-prefix is welcomed (EC-Clearinghouse, 1993; Smith et al, 1993).

Finally, the opportunities to study these conditions are limited because some of the changes described are acute, uncommon and respond readily to treatment (Glick et al, 1994; Robinson and Zakrzewska, 1994).

\subsubsection{Statistical issues}

\subsubsection{Multiple non-independent sites}

Periodontal epidemiology must also consider many measurements of the same variable. These are site-based measures of aetiological factors (plaque, restorations or specific microorganisms) and disease outcome as well as subject based factors such as age, tobacco and health status. Consequently there has also been discussion about whether the unit of inference should be the site or the subject.

One prominent example of the use of site-based inference was by the Forsyth group in the development of the burst theories of attachment loss (Haffajee et al, 1983; Socransky et al, 1984; Haffajee et al, 1985). Simple statistical techniques assume independent data, but the use of site-based units of inference ignores the mutual dependence of attachment loss at several sites in the same mouth on subject-based factors (Imrey, 1986). There is agreement that site-based inference detected many false positives and the statistical basis of the burst theories has been repudiated (Imrey, 1986; Ralls and Cohen, 1986; Gunsolley and Best, 1988; Sterne et al, 1990). Analytic techniques have been proposed which use the site as the unit of inference but consider the effect of subject based factors (Baelum et al, 1990; DeRouen et al, 1991; Albander and Goldstein, 1992), but these techniques are experimental and complex.

Imrey (1986) and Sterne et al. (1990) have recommended the use of subject-based units of inference, particularly if a subject-based factor is under investigation (e.g. HIV infection). Subject-based units are suitable for parametric and non-parametric analysis including regression with or without transformed variables but there is loss of precision (Sterne $e t$ al, 1990). For example, the use of intra-subject means swamps important detail if there are important changes at small numbers of sites. There is an additional problem that parametric analysis of a number of intra-subject means has a site-based unit of inference. 
To prevent swamping, Imrey (1986) proposed several methods for summarising important sites within an individual (i.e. summary measures) including:

i) the mean/medians of the $k$ largest sites with attachment loss

ii) the $k$ th percentile of site attachment level changes

iii) the proportion of sites with attachment loss above a given threshold

iv) clinical categorisation of response

where $k$ is small. It will be seen that: (i) measures the most severe site if $k=1$; (iii) is the extent of disease within a subject and (iv) becomes the presence of absence of a characteristic for a binary outcome.

The final consequence of the number of factors and outcomes involved in periodontal research is the number of possible combinations of variables which increases type 1 error. Type one error can be reduced by limiting the number of analyses to a small number of outcomes stated in advance.

\subsubsection{Measurement}

There are numerous measures and indices of periodontal health. Early indices aggregated signs of gingivitis and periodontitis. Recent measures have separated the two.

Periodontal indices are prone to poor reliability. The effects of poor measurement reliability can be reduced by the use of appropriate measures or indices and by calibration. The uncertainty created by poor reliability can be quantified during the study by comparing repeat examinations (Ramfjord, 1967; Barnes et al, 1986; Pihlstrom, 1992)

\subsubsection{1 $\quad$ Aggregate measures}

Aggregate measures were used extensively in early epidemiology of periodontal diseases but are used less now. Burt (1991) has discussed the problems of such indices and in particular Russell's periodontal index. His main concern was their biological validity, at the time gingivitis was believed to be the early stage of periodontitis and periodontitis was assumed to be generalised throughout the mouth. We know now that gingivitis is common but periodontitis is much less so, that most gingivitis does not become periodontitis and that periodontitis can be site-specific. In addition, the inclusion of gingivitis on the same scale created mathematical distortion of the findings. Group means were also calculated with Russell's PI and this swamped the shapes of distributions. 
The Community Periodontal Index of Treatment Need (CPITN) is a recent aggregate index whose biological validity has been challenged (Ainamo et al, 1982; Baelum et al, 1995). CPITN is not sufficiently precise for research (Burt, 1991) and is unsuitable for research with HIV since it ignores recession. Attachment loss in the absence of pocketing is characteristic of HIV-associated diseases (Winkler and Murray, 1987).

The only current alternative to aggregated measures are to measure probing depths, attachment loss and gingivitis separately (Burt, 1991). Partial scoring (simplified indices) reduces the burden to researcher and subject. They may include selected teeth, fewer sites on individual teeth or sections of the mouth (Ramfjord, 1967; Barnes et al, 1986). Partial scoring under-records attachment loss and plaque and gingivitis scores so researchers must decide whether to sacrifice sensitivity for expediency (Sterne $e t$ al, 1990; Bentley and Disney, 1995).

\subsubsection{Attachment levels and probing depths}

Periodontal attachment level is commonly recorded relative to a fixed reference point, usually the cemento-enamel junction (CEJ), by passing a fine probe down to the junctional epithelium (Pihlstrom, 1992). Measurement is made on visual scales or electronically. In longitudinal studies measuring change in attachment level, customised appliances (stents) are sometimes used to provide a reference point in both the long and horizontal axes of the teeth. Probing is complicated if the free gingiva obscures the CEJ. As a consequence, the probing depth is measured first, then the relative positions of the CEJ and gingival crest (Ramfjord, 1967). This has the advantage of recording both probing depth and recession (Griffiths et al, 1988), but adds problems of rounding error (Imrey, 1986).

Periodontal probing is not entirely satisfactory as a measurement method (Burt, 1991). Probing does not record the true site of the junctional epithelium and its validity changes with differing disease, probing force and probe width (Armitage et al, 1977; Lang, 1991; Theil and Heaney, 1991; Pihlstrom, 1992). To provide precise data many sites must be probed and this is uncomfortable and time-consuming (Watts, 1989).

Probing attachment loss (PAL) is not a reliable measure. Approximately $90 \%$ of recordings can be replicated within $1 \mathrm{~mm}$ (Badersten et al, 1984). This is a $2 \mathrm{~mm}$ range for a disease which infrequently destroys $6 \mathrm{~mm}$ of attachment in a lifetime. Reproducibility of probing changes with examiner (Smith et al, 1970), tooth type (Espeland et al, 1991), probing force (Mullaly and Linden, 1994), position of probe (Watts, 1989), site of exam (Kingman et al, 1991), probing depth (Janssen et al, 1988) and position of probe markings (Watts, 1989) 
and can be increased with calibration, constant force probes, stents, appropriate marking, the use of the mean value of repeated examinations and automated probes (Smith $e t$ al, 1970; Magnusson et al, 1988; Watts, 1989; Watts, 1989; Espeland et al, 1991; Mullaly and Linden, 1994).

\subsubsection{3 $\quad$ Gingivitis}

Indices of gingival health have been reviewed extensively (Barnes $e t a l, 1986$; Grant $e t a l$, 1988; Greene, 1990). Current indices relate to gingival colour and oedema (Suomi and Barbano, 1968), bleeding on probing (Saxer and Muhlemann, 1975) or both (Muhlemann and Mazor, 1958; Löe and Silness, 1963). 'Gingival indices' which consider recession or pocketing are aggregate indices (see above) (Schour and Massler, 1958).

The Gingival Index of Löe and Silness (Löe and Silness, 1963) has been used extensively and successfully in surveys and experimental epidemiology, has explicit and mutually exclusive categories, is precise, lends itself to calibration and incorporates the presence of bleeding on probing (Alexander et al, 1971; Griffiths et al, 1988; Greene, 1990).

The reliability of gingival indices has received less research attention than measures of attachment loss. Griffiths et al. (1988) expressed concern about reliability but Smith et al. (1970) found the subjective measures were as reliable as more objective measures. Reliability improves with training and calibration (Smith et al, 1970; Alexander et al, 1971). Gingival indices are less reliable under field conditions (Kingman $e t a l, 1991)$ and bleeding on probing is less reproducible in deeper pockets (Watts, 1987).

\subsubsection{Plaque}

Plaque indices have been used to record the extent (Greene and Vermillion, 1960; Quigley and Hein, 1962; Greene and Vermillion, 1964; Ramfjord, 1967; Turesky et al, 1970), thickness (Silness and Löe, 1964) or weight (Grant et al, 1988) of plaque on a tooth.

The plaque index of Silness and Löe is sensitive to small changes, can be transformed easily to record the presence or absence of plaque and is widely used in epidemiological research (Greene, 1990). This index has subjective criteria and should be used by experienced and trained examiners (Mandel, 1974). 


\section{CHAPTER 4 DEVELOPMENT AND RELIABILITY OF DIAGNOSTIC CRITERIA FOR PERIODONTAL CHANGES ASSOCIATED WITH HIV INFECTION}

\subsection{Abstract}

\section{Objectives}

i, Develop diagnostic criteria for HIV-associated periodontal changes and assess their ability to improve inter-examiner reliability.

ii, Identify how a panel of experts make diagnoses of HIV-associated periodontal changes .

\section{Design}

Formal experimental with pre-and post-testing of subjects randomly assigned to study and control groups.

\section{Method}

Recording signs and symptoms seen on clinical slides

\section{Participants}

5 oral medicine specialists and 4 periodontists of a university stomatology department with clinical and/or research experience of $\mathrm{HIV}$-associated periodontal diseases.

\section{Outcome measures}

Reliability of recognition of clinical signs, signs associated with diagnoses and reliability of diagnoses.

\section{Results}

Kappas for recognising clinical signs ranged between -0.04 and 0.75 . Signs associated with diagnoses did not correspond with classical characterisation of diseases. Diagnostic reliability improved in the study group more than the control group $(P=0.011$, Wilcoxon rank sum) and reached a higher level than the controls $(P=0.005)$.

\section{Conclusions}

The examiners showed only fair reliability in the recognition of clinical signs, made diagnoses intuitively and had only fair agreement on the diagnosis of periodontal diseases. The inter-examiner reliability of examiners trained and calibrated in the use of the criteria increased and was greater than among untrained examiners. 


\subsection{Introduction}

Our understanding of HIV-associated periodontal conditions is limited because research has been hindered by the lack of suitable diagnostic criteria. One group reviewed the oral manifestations of HIV disease, yet their illustrations were of conventional periodontitis and others have used indices insensitive to the changes described in the infection (Scully et al, 1990; Lucht et al, 1991).

Diagnosis involves 3 stages: clinical signs and symptoms must be recognised; the clinician must be aware of the character of relevant diseases and finally, the observed signs must be assimilated into a pattern corresponding to a disease. Diagnostic reliability might be improved by acting at any of these stages: calibration improves agreement on the recognition of signs; simple and explicit diagnostic criteria aid the clinician's knowledge and training standardises assimilation into a diagnosis.

This chapter describes the development of diagnostic criteria for HIV-associated periodontal conditions. The project aimed to investigate the way a panel of experts made diagnoses of HIV-associated periodontal changes and to assess whether the new diagnostic criteria improved inter-examiner reliability.

\subsection{Methodology}

\subsubsection{Development of the criteria}

A set of diagnostic criteria for HIV-associated periodontal conditions were devised based on a literature review and the experience of three dentists who provided care for people with HIV (Guy Palmer, United Medical and Dental Schools, London; Peter Robinson, University College Dental Hospital, London and James Winkler, University of California at San Francisco)(Table 4.1). The criteria reflected descriptions of the conditions in the literature which were modified to increase objectivity. Their validity was assessed by comparison with clinical photographs and by examining a small number of patients with HIV. The criteria were evaluated and refined for ease of use and objectivity in informal tests by the London HIV and Dentistry Group.

The criteria distinguished seven conditions possibly found in people with HIV. Precision was sacrificed to simplify the criteria by ignoring uncommon diseases and by assuming that gingivitis in the presence of attachment loss was periodontitis. This kind of assumption is used in epidemiology but makes the criteria unsuitable for diagnosis in individual patients (Tan et al, 1982). 
The criteria were arranged in a series of tests of increasing stringency and arranged in a flow chart (Fig. 4.1). Since one site might manifest signs of several conditions, some of the criteria could be combined. For example, it was possible to diagnose HIV-associated changes super-imposed on sites with conventional periodontitis.

\subsubsection{Assessment of the reliability of the criteria.}

The reliability of the criteria were assessed using a formal experimental design involving pre- and post-testing of subjects randomly assigned to study and control groups.

\subsubsection{Selection of populations}

The intended sample were dentists experienced in periodontal examination of people with HIV. Sufficient examiners were required to lend power to the study to detect any benefit of calibration and training.

The examiners were nine dentists (five oral medicine specialists and four periodontists) working in a university stomatology department. Each had worked in the department for more than one year, was familiar with the literature on HIV-associated periodontal conditions and had provided care for patients at risk of them. Several examiners had published research on periodontal changes in HIV infection. One possible examiner (the head of an oral medicine team) was not invited to participate in case a hierarchical relationship affected the calibration exercise.

\subsubsection{Variables}

The variables were: the recognition of signs of periodontal health; the signs the examiners associated with diagnoses and the inter-examiner reliability of diagnoses.

\subsubsection{Selection and development of measures}

A data sheet was developed to be completed by each examiner for each slide and enquired into the presence of all the clinical signs and diagnoses incorporated into the diagnostic criteria (Appendix 1). The sheet was standardised to give no guidance about specific 
Table 4.1. Diagnostic Criteria for studies of HIV-associated periodontal changes.

The following diagnostic criteria are for use in the diagnosis of periodontal conditions in epidemiological studies. The criteria allow diagnosis of several conditions in the same mouth. It should be possible, by studying each quadrant, sextant or tooth at a time, to diagnose conventional periodontitis and HIVassociated Periodontitis in the same subject.

\section{Chronic marginal gingivitis}

Defined by the presence of erythema, glazing or swelling of the free gingival margin.

(With no recession except at the mid-buccal surfaces and no pocketing greater than $3 \mathrm{~mm}$.)

\section{Necrotising ulcerative gingivitis (acute)}

Defined by the presence of conventional gingivitis and both of the following

i) gingival ulceration radiating from one or more inter-dental papilla tips

ii) inflammation limited to the marginal gingiva

\section{$3 \quad$ HIV-associated gingivitis}

Defined by the presence of gingival erythema manifested by one of the following:

i) punctate erythema of the attached gingiva, ignoring lesions limited to the marginal gingiva

ii) diffuse erythema of the attached gingiva, ignoring lesions limited to the marginal gingiva

iii) a well-defined red band along the free gingival margin which does not bleed in $50 \%$ or more sites on probing.

\section{Ulcerative HIV-associated gingivitis}

Defined by the presence of HIV-associated gingivitis and one of the following:

i) ulceration of the free gingiva

ii) spontaneous bleeding.

$5 \quad$ Chronic adult periodontitis

Defined by the presence of gingivitis and attachment loss manifested by one of the following:

i) recession exposing the $\mathrm{CEJ}$ (excluding the mid-buccal surfaces)

ii) pocketing $\geq 4 \mathrm{~mm}$ in two or more sites

Recession at the mid-buccal point of the tooth may be due to abrasion and is ignored

6 Ulcerative HIV-associated periodontitis

Defined by the presence of HIV-associated gingivitis and attachment loss where the recession is $\geq 2 \mathrm{x}$ the probing depth (excluding the mid-buccal surfaces) and one of the following:

i) presence of exposed bone

ii) ulceration/necrosis of the attached gingiva

iii) patient complaint of severe, "deep" or "bone" pain* 


\title{
Table 4.1 continued
}

\author{
7 Non-ulcerative HIV-associated periodontitis \\ Defined by the presence of HIV-associated gingivitis and attachment loss where the recession is $\geq 2 \times$ the \\ probing depth (excluding the mid-buccal surfaces) in one of two forms: \\ i) reverse architecture (i.e., a depression in place of an inter-dental papilla) \\ ii) cratering (a deepening of the inter-dental col where the buccal and palatal/lingual gingivae remain).

\section{$8 \quad$ Necrotizing periodontitis}

Defined by the presence of ulceration/necrosis of the attached gingiva and mucosa and/or bone $10 \mathrm{~mm}$ from the cemento-enamel junction or beyond the muco-gingival junction, whichever is nearer.

Attachment loss can be recorded in two forms; with or without gingivitis. The presence of attachment loss in the absence of gingivitis is not intended to imply active disease. Attachment loss with marginal gingivitis will be assumed to be chronic adult periodontitis (See 5 above).

\section{Attachment loss with reverse architecture or inter-dental cratering}

Defined by the presence of attachment loss $\geq 4 \mathrm{~mm}$ where the recession is $\geq 2 \times$ the probing depth (excluding the mid-buccal surfaces) in one of two forms:

i) reverse architecture (i.e., a depression in place of an inter-dental papilla)

ii) cratering (a deepening of the inter-dental col where the buccal and palatal/lingual gingivae remain).

\section{Generalised attachment loss}

Defined by the presence of attachment loss $\geq 4 \mathrm{~mm}$ (excluding the mid-buccal surfaces) without reverse architecture and or cratering. 

Figure 4.1. Decision making flow chart for diagnosis of periodontal conditions
associated with HIV

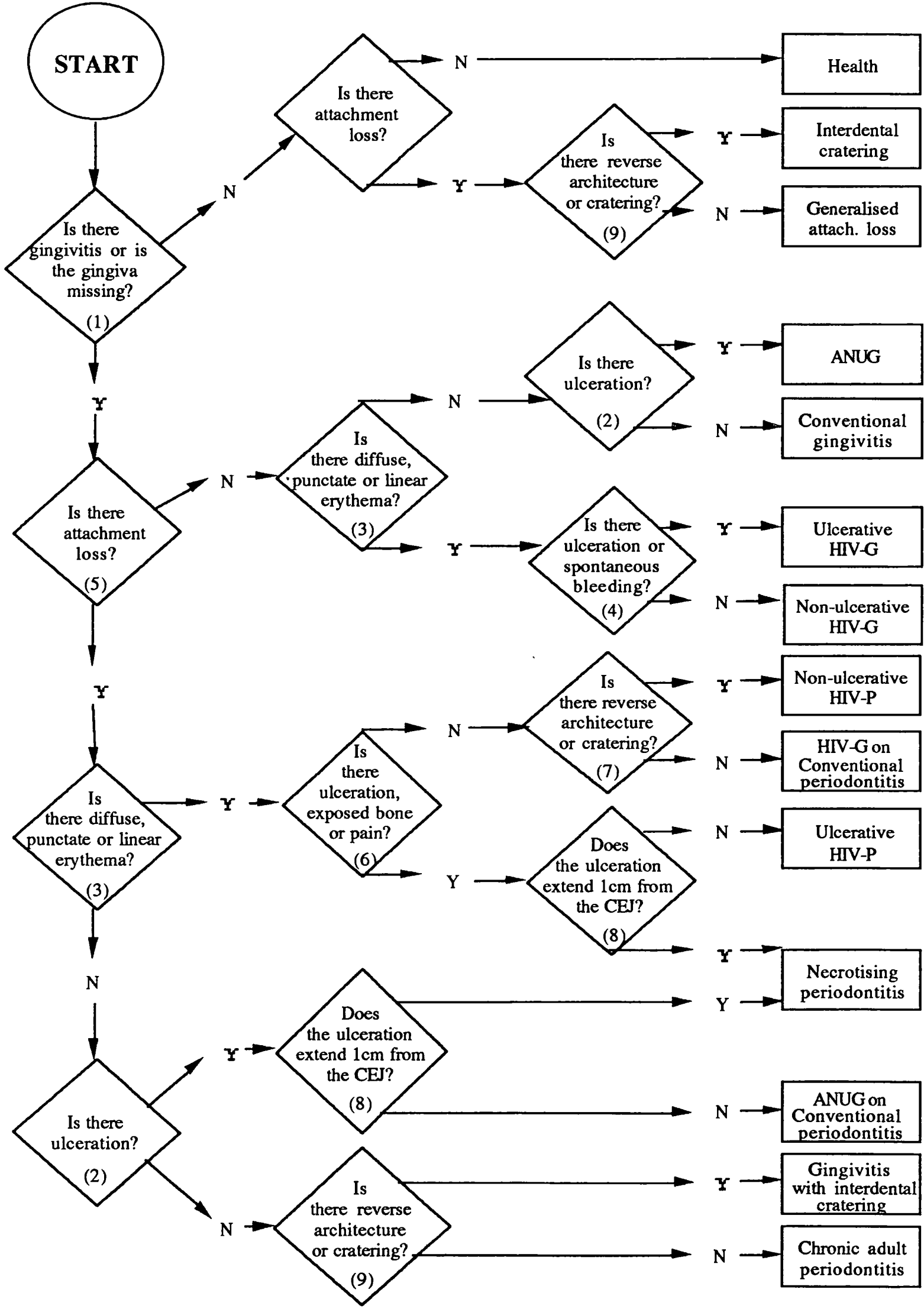

Numbers in parentheses refer to the number of the criterion 
photographs. Closed questions were used to allow comparison between examiners although space was provided to allow examiners to add signs or diagnoses not on the sheet.

\subsection{6}

\section{Data collection - Conduct}

Initially (Stage 1), all the examiners were shown a series of 20 clinical photographs as colour transparencies projected onto a white screen in a darkened room. The photographs were accompanied by brief vignettes containing the age of the patient (all men), a brief clinical history and clinical information which could not be elicited from a photograph (e.g., probing depths and bleeding on probing). Examiners were given unlimited time with each photograph but asked not to discuss them.

Each examiner was asked to identify the clinical signs in part of the photograph by answering eleven closed questions or by providing alternative answers. Examiners recorded a diagnosis for each photograph by ticking one of 14 possible diagnoses or by providing an alternative (see Appendix 1). Examiners were asked to ensure they answered every question and always made a diagnosis.

After Stage 1, five examiners (three oral physicians and two periodontists) were selected randomly from the 9 examiners to form a study group. The remaining four became the control group. Each member of the study group was asked to study a copy of the criteria. The group met once for 2 hours. Training consisted of instruction in the use of the criteria and calibration by comparing diagnoses for a new set of clinical photographs.

One week later (Stage 2), all the examiners were shown the original photographs in a different order, asked to identify the signs and to record diagnoses.

\subsection{7}

\section{Data analysis.}

The Stage 1 data were sorted by the diagnosis made and the conditions grouped by the strength of association with gingival ulceration. Chi square analyses were used to compare the signs associated with each diagnosis within each group and to identify differences between the ulcerative and non-ulcerative forms of HIV-associated periodontitis (HIV-P). Inter-examiner reliability for the identification of clinical signs was calculated using the kappa statistic for dichotomous variables.

Diagnostic agreement was calculated from the proportion of examiners who agreed on each photograph. Changes in agreement among the study and control groups, and the differences between the groups, were compared using the Wilcoxon rank sum statistic. 


\subsection{Results}

\subsubsection{Signs most commonly associated with each diagnosis}

Table 4.2 shows the frequency of diagnoses for each of the 20 slides in the Stage 1 review. Table 4.3 reports the frequency with which the examiners associated clinical signs with each of the diagnoses. Chronic marginal gingivitis (CMG), HIV-associated gingivitis (HIV-G) without ulceration, and HIV-P without ulceration were rarely associated with ulceration. CMG and HIV-G without ulceration were associated with similar clinical signs. Significant differences $(P<0.05$, Chi sq.) in the signs associated with these conditions were:

1) HIV-P without ulceration was often associated with marginal gingiva of normal colour, whereas CMG and HIV-G without ulceration were not.

2) Punctate erythema was associated with HIV-G without ulceration more often than with CMG.

3) A distinct red band of the marginal gingiva was more often associated with CMG than with HIV-G or HIV-P without ulceration.

4) Gingival swelling was more often associated with CMG than with HIV-P without ulceration.

5) Recession was more often associated with HIV-P without ulceration than with CMG or HIV-G without ulceration. 
Table 4.2. Diagnoses for 20 clinical slides made by 9 examiners

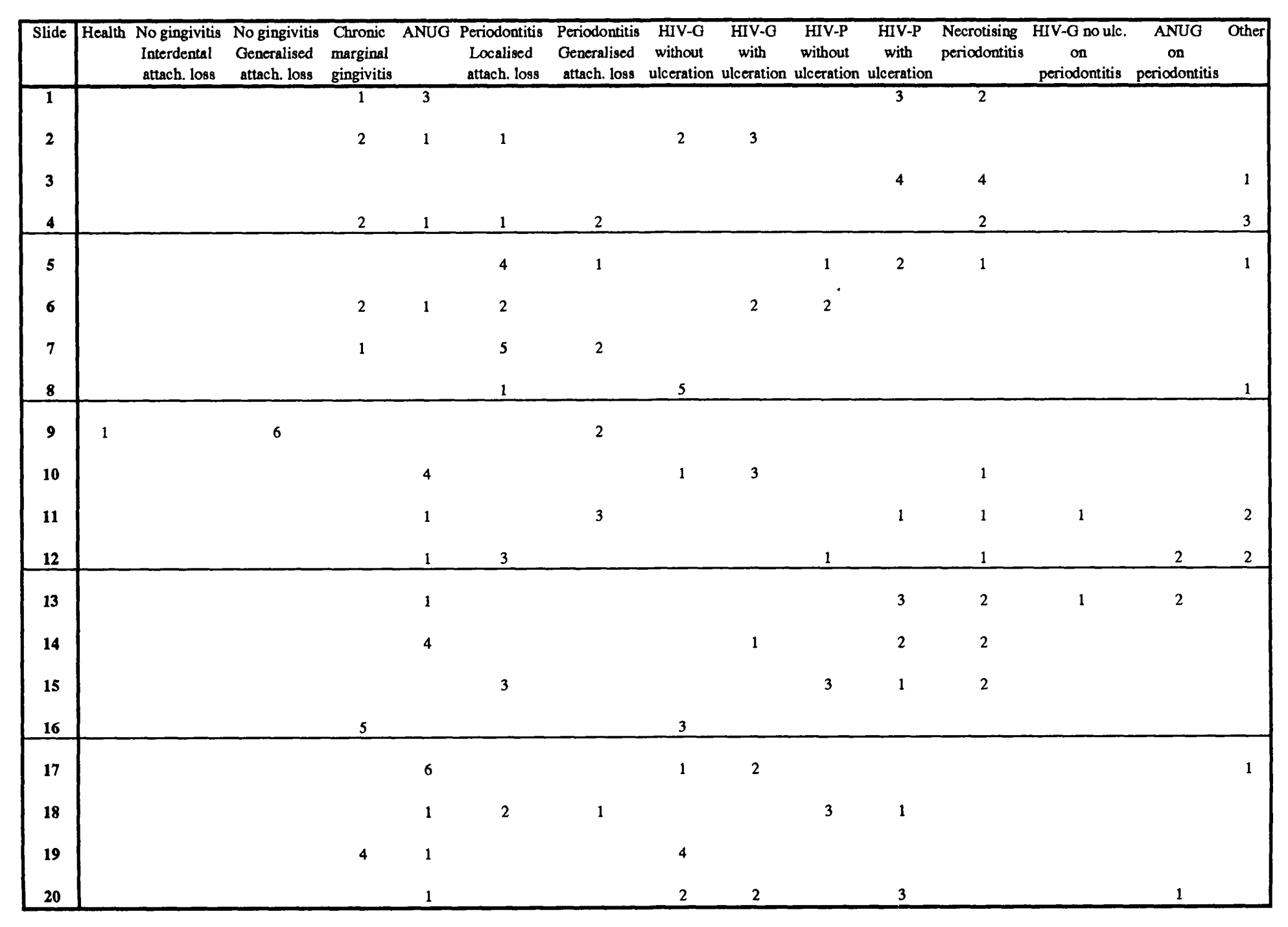


Table 4.3. Clinical signs associated with diagnoses by 9 examiners

\begin{tabular}{|c|c|c|c|c|c|c|c|c|c|c|c|c|}
\hline \multirow[b]{2}{*}{$\begin{array}{c}\text { Diagnosis } \\
\text { (n) }\end{array}$} & \multicolumn{3}{|c|}{ Marginal gingiva } & \multicolumn{3}{|c|}{\begin{tabular}{|l} 
Attached gingiva \\
\end{tabular}} & \multirow[b]{2}{*}{$\begin{array}{c}\text { Red band } \\
\mathrm{n}(\%)\end{array}$} & \multirow[b]{2}{*}{$\begin{array}{c}\text { Swelling } \\
\mathrm{n}(\%)\end{array}$} & \multirow{2}{*}{$\begin{array}{c}\text { Spontan. } \\
\text { bleeding } \\
\mathrm{n}(\%)\end{array}$} & \multirow[b]{2}{*}{$\begin{array}{c}\text { Recession } \\
\mathrm{n}(\%)\end{array}$} & \multirow{2}{*}{$\begin{array}{l}\text { Ulc. marginal } \\
\text { gingiva } \\
n(\%)\end{array}$} & \multirow{2}{*}{$\begin{array}{l}\text { Ulc. attached } \\
\text { gingiva } \\
n(\%)\end{array}$} \\
\hline & $\begin{array}{c}\text { Fiery red } \\
\mathrm{n}(\%)\end{array}$ & $\begin{array}{l}\text { Red } \\
\mathrm{n}(\%)\end{array}$ & $\begin{array}{c}\text { Normal } \\
\mathrm{n}(\%)\end{array}$ & \begin{tabular}{|c} 
Diff. red \\
$\mathrm{n}(\%)$
\end{tabular} & $\begin{array}{c}\text { Punct. red } \\
\mathrm{n}(\%)\end{array}$ & $\begin{array}{c}\text { Normal } \\
\mathrm{n}(\%)\end{array}$ & & & & & & \\
\hline CMG (17) & $5(30)$ & $11(65)^{*}$ & $0(0) *$ & $8(47)$ & $2(12)^{*}$ & $6(35)$ & $14(82)^{*} \dagger$ & $17(100)^{*}$ & $0(0)$ & $5(29) \dagger$ & $4(24)$ & $1(6)$ \\
\hline HIVG-Ulc (18) & $7(39)$ & $10(56) \dagger$ & $0(0) \dagger$ & $10(56)$ & $5(28)^{*}$ & $2(11)$ & $9(50)^{*}$ & $16(89)$ & $3(17)$ & $3(17)^{*}$ & $0(0)$ & $0(0)$ \\
\hline HIVP-Ulc (10) & $1(10)$ & $2(20)^{*}+$ & $9(90)^{*}+\ddagger$ & $3(30)$ & $2(20)$ & $6(60)^{*}$ & $2(20) \dagger \ddagger$ & $7(70)^{*} \dagger$ & $1(10)^{*}$ & $9(90)+*$ & $1(10)^{*}$ & $0(0)^{*}$ \\
\hline ANUG (26) & $9(35)$ & $16(62)$ & $2(8)$ & $15(58)$ & $6(23)$ & $6(23)$ & $11(42)$ & $22(85)$ & $11(42)$ & $16(62) \ddagger$ & $24(92)$ & $3(12) \dagger \ddagger$ \\
\hline HIVG+Ulc (13) & $6(46)$ & $4(31)$ & $1(8)$ & $8(62)$ & $2(15)$ & $2(15)$ & $5(38)$ & $13(100)$ & $5(38)$ & $4(31) \S 9$ & $13(100)$ & $2(15) \S \#$ \\
\hline HIVP+Ulc (20) & $9(45)$ & $8(40)$ & $0(0) \ddagger$ & $9(45)$ & $7(35)$ & $1(5)^{*}$ & $11(55) \ddagger$ & $17(85) \dagger$ & $9(45)^{*}$ & $15(75)$ & $19(95)^{*}$ & $14(70) \# \ddagger^{*}$ \\
\hline Nec perio(18) & $6(33)$ & $9(50)$ & $1(6)$ & $9(50)$ & $7(39)$ & $1(6)$ & $7(39)$ & $14(78)$ & $12(67)$ & $18(100) \S \ddagger$ & $13(72)$ & $10(56) \S \dagger$ \\
\hline \multicolumn{7}{|c|}{$\mathrm{CMG}=$ Chronic marginal gingivitis } & \multicolumn{6}{|c|}{ Diff. = Diffuse } \\
\hline \multicolumn{7}{|c|}{ HIVG-UIc = HIV-Gingivitis without ulceration } & \multicolumn{6}{|c|}{ Punct $=$ Punctate } \\
\hline \multicolumn{7}{|c|}{ HIVP-Ulc = HIV-Periodontitis without ulceration } & \multicolumn{6}{|c|}{ Spontan. $=$ Spontaneous } \\
\hline \multicolumn{7}{|c|}{ ANUG = Acute necrotising ulcerative gingivitis } & \multicolumn{6}{|c|}{ Ulc $=$ Ulcerated } \\
\hline
\end{tabular}

HIVP+Ulc $=$ HIV-Periodontitis with ulceration

$\mathrm{Nec}$ Perio $=$ Necrotising periodontitis

$*, \uparrow, \ddagger, \S, \uparrow$ and \# denote $\mathrm{P}<0.05$ (Chi sq.) for differences within each column

Pocketing, mucosal ulceration and exposure of bone were associated similarly with all the diagnoses 
ANUG, HIV-G with ulceration, HIV-P with ulceration and necrotising stomatitis were all associated with swelling and ulceration of the gingiva. The perceived differences between these conditions were:

1) Necrotising stomatitis and HIV-P with ulceration were associated with recession more than was HIV-G with Ulceration. Necrotising stomatitis was more often associated with recession than was ANUG.

2) Necrotising stomatitis and HIV-P with ulceration were both associated with ulceration of the attached gingiva more than were HIV-G with ulceration or ANUG.

Different signs were associated with the two forms of HIV-P. As well as lacking ulceration of the marginal or attached gingiva, HIV-P without ulceration was less often associated with a red band of the marginal gingiva, swelling or spontaneous bleeding than was HIV-P with ulceration (all $P<0.05$, Chi sq.).

\subsubsection{Reliability of recognition of clinical signs.}

The Kappa statistic is used to measure agreement for dichotomous variables. Kappas range between -1 and +1 with a score of zero representing only random agreement. Scores below 0.4 represent poor agreement and above 0.7 represent good agreement. Between these scores agreement is said to be fair/good.

Table 4.4 presents the Kappa scores for the agreement on identification of clinical signs for the study and control groups at both stages of data collection. Variables for which several choices were offered to examiners have been dichotomised (for instance, the data for fiery red, red, normal or other in the appearance of the marginal gingiva) .

Generally the reliability for all variables was fair. In Stage 1 the study group showed greater agreement than the control group in the recognition of increased probing depth. In Stage 2 the study group showed greater reliability for two variables (colour of marginal gingiva and probing depth).

\subsubsection{Reliability of diagnosis}

The proportion of subjects agreeing on the diagnosis for each slide in the Stage 1 and 2 reviews was tabulated for the study and control groups. The differences in the number of reviewers agreeing before and after the calibration session were determined and analysed by 
the Wilcoxon rank-sum test. The median change in agreement level between the two reviews was 0.0 in the control group and 0.3 in the study group $(p=0.0113)$, indicating that the training had improved the level of agreement.

The proportion of subjects in the study and control groups who agreed on the diagnosis of each slide in the stage 2 review was also analysed by the Wilcoxon test. The median agreement for the control group was 0.33 and for the study group, $0.60(p=0.0054)$, indicating a significantly higher level of agreement in the trained group.

Frequency of diagnoses by rank of diagnosis in the original, control and study groups are expressed graphically in Appendix 2.

\subsection{Discussion}

The findings of this study are compatible with our knowledge of diagnosis. Examiner reliability is often poor, but improves with training and calibration (Smith et al, 1970; Alexander et al, 1971; Cowell et al, 1975). The use of photographs may have limited the information available to the examiners and reduced their ability to diagnose accurately. However, this technique is useful for calibration for acute conditions which are uncommon and painful. Moreover, the use of photographs standardised the examination.

\subsubsection{Signs associated with diagnoses}

Examiners associated similar clinical signs with chronic marginal gingivitis and HIVassociated gingivitis (Table 4.3). The only signs associated with these conditions with different frequency were punctate erythema of the attached gingiva ( $12 \%$ and $28 \%$ of diagnoses, respectively) and a distinct red band of the gingiva (82\% and $50 \%$ of the diagnoses, respectively). Thus, with differences between the frequencies of $16 \%$ and $32 \%$, in only a maximum of $48 \%$ of cases can the distinction between these two conditions be attributed to these two variables. This figure is an over-estimate since examiners more commonly associated red bands with conventional gingivitis than with HIV-gingivitis. Distinct red bands are strongly associated with HIV-gingivitis in the literature (Winkler $e t$ al, 1989)) suggesting that diagnoses were made using other criteria.

Examiners readily distinguished HIV-P with and without ulceration. HIV-P without ulceration lacked the marginal red bands, swelling and spontaneous bleeding which they associated with the ulcerated form. The distinction between HIV-P with and without ulceration is new and was introduced to reflect two different presentations in the literature 
Table 4.4. Kappa scores for inter-rater agreement on signs of periodontal diseases

\begin{tabular}{|c|c|c|c|c|}
\hline \multirow[b]{2}{*}{ Sign } & \multicolumn{2}{|l|}{ Stage 1} & \multicolumn{2}{|c|}{ Stage 2} \\
\hline & Study Gp (95\% CI) & Control Gp (95\% CI) & Study Gp (95\% CI) & Control Gp (95\% CI) \\
\hline Red marginal gingiva & $0.39(0.24,0.53)$ & $0.64(0.46,0.82)$ & $0.57(0.42,0.72) \dagger$ & $0.01(-0.17,0.19) \dagger$ \\
\hline Red attached gingiva & $0.48(0.33,0.63)$ & $0.50(0.33,0.69)$ & $0.53(0.38,0.68)$ & $0.46(0.28,0.64)$ \\
\hline Red band & $0.45(0.31,0.63)$ & $0.38(0.19,0.56)$ & $0.59(0.45,0.74)$ & $0.44(0.27,0.62)$ \\
\hline Swelling & $0.31(0.17,0.44)$ & $0.21(0.03,0.39)$ & $0.17(0.03,0.31)$ & $0.19(0.01,0.37)$ \\
\hline Spontaneous bleeding & $0.33(0.18,0.48)$ & $0.53(0.34,0.72)$ & $0.63(0.44,0.82)$ & $0.30(0.11,0.49)$ \\
\hline Increased probing depth & $0.75(0.60,0.89)^{*}$ & $0.31(0.12,0.50)^{*}$ & $1.00(0.84,1.00) \ddagger$ & $0.24(0.06,0.41) \ddagger$ \\
\hline Recession & $0.50(0.34,0.67)$ & $0.30(0.08,0.52)$ & $0.71(0.55,0.88)$ & $0.76(0.54,0.98)$ \\
\hline Ulceration marginal gingiva & $0.51(0.37,0.65)$ & $0.71(0.52,0.90)$ & $0.95(0.81,1.00)$ & $0.64(0.46,0.82)$ \\
\hline Ulceration attached gingiva & $0.38(0.23,0.62)$ & $0.27(0.07,0.46)$ & $0.57(0.42,0.71)$ & $0.59(0.40,0.82)$ \\
\hline Ulceration mucosa & $0.48(0.34,0.62)$ & $0.48(0.27,0.69)$ & $0.74(0.59,0.88)$ & $0.64(0.45,0.82)$ \\
\hline Exposed bone & $0.08(-0.06,0.23)$ & $-0.04(-0.23,0.16)$ & $-0.01(-0.16,0.13)$ & $-0.01(-0.20,0.17)$ \\
\hline
\end{tabular}

$: t$, and $\ddagger$ indicate $p<0.05$ using the $95 \%$ confidence intervals. 
(Greenspan et al, 1986). Whilst it is possible that the lesions which the examiners termed 'HIV-periodontitis without ulceration' represented a destructive disease, another explanation is they are areas of arrested destruction. In that case, the term 'periodontitis' is a misnomer and 'localised inter-dental attachment loss' might be more appropriate.

A distinction between these two presentations might account for the microbiological differences between 'HIV-P' and ANUG. Localised areas of interproximal cratering and minimal pockets would be expected to have different microflora from lesions with progressive ulceration, though both might be termed 'HIV-P' from the early descriptions. The same misclassification allows over-estimation of disease prevalence.

Examiners associated all the ulcerative changes with swelling and ulceration of the gingiva. Necrotising Stomatitis and HIV-P with ulceration were associated with more extensive tissue damage (as evidenced by recession in Table 3 ) but there were no other differences in the features associated with the diagnoses. These data suggest that diagnoses were not made by use of criteria common to all examiners. A hypothesis supported by the poor agreement on diagnoses.

Other signs or a particular combination of signs may have led examiners to a particular diagnosis for each photograph. However, the data do not suggest this and the poor agreement on identification of signs and on diagnoses further suggests that diagnoses were made intuitively.

\subsubsection{Reliability of recognising signs}

In both stages of this study, examiners showed only fair reliability identifying clinical signs such as redness of the marginal gingiva or ulceration (Table 4). This emphasises the need for careful calibration before any study involving several examiners.

After calibration, the study group agreed on the colour of the marginal gingiva more than the controls. This improvement is encouraging but constitutes only one variable of eleven studied. Alexander (1971) did not detect any improvement in agreement until there had been three calibration sessions. Calibration in this study involved only reading of the criteria and one two hour session with photographs; thus, profound improvements were not expected. 


\subsubsection{Reliability of diagnosis}

The intuitive diagnoses and mediocre agreement on the recognition of signs contributed to the poor diagnostic agreement in the initial stage of this study (Table 2). The implications of this are important. Authors who have reported on HIV-associated periodontal conditions might not have been looking at this range of conditions at all. It must be stressed that the examiners in this study were 'experts' who were experienced and well informed. All worked in the same department of a dental school and it is likely that there is greater agreement among this group than among less experienced examiners not working in such close proximity.

There was significant improvement in the inter-examiner reliability of examiners who were trained and calibrated in the use of the diagnostic criteria, with no improvement among noncalibrated controls. This resulted in significantly more agreement among the calibrated group. These findings confirm the value of training and demonstrate the reliability of the criteria.

\subsubsection{Validity of the criteria}

The criteria were based on the experience of three clinicians and on a literature review. Criteria can be agreed at a consensus conference with many centres represented, but previous conferences have not produced mutually exclusive and objective criteria (EECClearinghouse, 1991; Greenspan et al, 1992). Given the conflicting reports of HIVassociated periodontal changes, these criteria were proposed as an interim measure. The best way to study periodontal health in HIV infection is to record standard measures in large samples of infected people. In the meantime, simple criteria were required for epidemiological studies where comprehensive periodontal measurements were impractical.

\subsubsection{Simplicity of the criteria}

The flow chart was designed to formalise the decisions used to make diagnoses. It uses information routinely collected during examination which should be incorporated into any diagnosis. Earlier research has employed comprehensive criteria, but which were not mutually exclusive (Riley et al, 1992).

The flow chart need not be memorised, but studied during training. Training is an essential component of any periodontal study (Smith et al, 1970; Cowell et al, 1975) but has only been reported in three studies of periodontal health and HIV infection (Melnick et al, 1989; 
Swango et al, 1991; Riley et al, 1992). Examiners may be resistant to training and calibration, but this may be reflected in the conflicting findings of research. This study has demonstrated that they are effective for this range of conditions.

\subsection{Conclusions}

Diagnostic criteria for HIV-associated periodontal changes were developed by 3 dentists.

A group of experienced examiners showed only fair reliability in the recognition of clinical signs and made diagnoses intuitively. The signs they associated with some periodontal changes did not correspond with characterisations of those changes. This undermines earlier microbiological and epidemiological research of HIV-associated periodontal diseases.

The examiners reached only fair diagnostic agreement. The inter-examiner reliability of examiners trained and calibrated in the use of the diagnostic criteria increased and was greater than among untrained examiners.

Rigid diagnostic criteria are an important feature of any epidemiological study. Interexaminer reliability will be increased if examiners are trained in their use and calibrated in the recognition of clinical signs. 
Chapter 5

\section{A CROSS-SECTIONAL STUDY OF THE PERIODONTAL HEALTH OF HOMOSEXUAL MEN WITH HIV INFECTION}

\section{$5.1 \quad$ Abstract}

\section{Objective}

Identify types, prevalence and severity of periodontal changes associated with HIV infection.

\section{Design}

Cross-sectional controlled blinded study.

\section{Setting}

Open access genito-urinary medicine clinic.

\section{Participants}

Convenience sample of 794 homosexual men aged 18-65.

\section{Outcome measures}

Prevalence, extent and severity of probing attachment loss (PAL), pocketing, gingival ulceration, gingivitis, bleeding on probing (BOP), gingival red bands and erythema of the attached gingiva (selected a priori).

\section{Results}

Prevalences in men with $(\mathrm{n}=312)$ and without HIV $(\mathrm{n}=260)$ were: PAL, 59.6\% and $28.5 \%$ respectively $\left(P<0.001, \mathrm{Chi}^{2}\right.$ ) ; pocketing, $51.0 \%$ and $31.9 \%(P<0.001)$; BOP, $96.5 \%$ and 92.3\% ( $P<0.038)$; gingival ulceration, $3.2 \%$ and $1.0 \%$ ( $P<0.031)$; red banding, $12.2 \%$ and $10.0 \%(P<0.410)$; diffuse erythema, $12.5 \%$ and $3.1 \%(P<0.001)$ and punctate erythema, 9.6\% and $1.1 \%(P<0.001)$. Decreased CD4 lymphocyte counts predicted the presence, extent and severity of PAL but not pocketing. Oral candidiasis predicted the extent and severity of gingivitis and the presence of diffuse and punctate erythema $(P=0.037,0.011$, 0.002 and $<0.001$ ).

\section{Conclusions}

Lost periodontal attachment is associated with HIV disease progression. Pocketing is associated with HIV but not disease progression. Gingival ulceration is associated with HIV but gingivitis and erythema of the attached gingiva are associated with oral candidiasis. 


\section{$5.2 \quad$ Introduction}

A number of periodontal changes have been associated with HIV infection. Little is known about the changes or of risk factors for them. Methodological issues in the studies that have been conducted may have hindered progress. Samples have been self-selected, too small and uncontrolled. Measures and indices of health have been used which are insensitive to the changes expected (Robinson, 1992; Lamster et al, 1995 and Chapters 2 and 4). Therefore, this study aimed to assess the types, prevalence and severity of periodontal changes in people with HIV infection and to identify factors that may cause them.

\subsection{Method}

\subsubsection{Selection of populations}

A control group was required to provide a yardstick of the background disease in the population and blinding was used to reduce measurement bias (Sitthi-amorn and Poshyachinda, 1993). This study was restricted to homosexual men to remove confounding effect by sex, sexual behaviour or unforeseen effects associated with injected drug use. Homosexual men were targeted since they constitute the majority of cases of HIV infection in southern England.

The study was conducted at a genito-urinary medicine clinic with numbers of homosexual men with and without HIV infection, who were similar in other respects and whose medical records were available. Use of a non-dental setting reduced selection bias.

The working hypothesis was that HIV disease would be associated with accelerated periodontal attachment loss which would be more evident after the disease had been present for some time. The annual incidence of gingival ulceration among people with HIV is approximately $2 \%$ with a significant relapse rate (Glick et $a l, 1994$; Robinson and Zakrzewska, 1994). It was estimated that this might result in moderate attachment loss in $10 \%$ of men in late HIV disease, in addition to background levels of disease evident in adults (Brown et al, 1989; Todd and Lader, 1991). A sample of 300 men was chosen to give an $85 \%$ power to detect a difference between proportions of 0.1 and 0.2 between 'early' and 'late' HIV disease for an alpha of 0.05 (Altman, 1991). Estimates of the prevalence of HIV infection among homosexual men at the clinic suggested that a convenience sample of 800 homosexual men would include 300 men with HIV and a similar number of uninfected men. Convenience sampling was chosen to provide the largest sample in the time available. Other commitments of the researcher limited attendance to Thursdays and Fridays. 
The inclusion criteria for the project were: men between 18 and 65 years of age, with 20 or more teeth, no history of injected drug use and without medical conditions which precluded periodontal examination. Men with Downs syndrome, diabetes or leukaemia or taking epanutin or cyclosporins were excluded.

To prevent selection bias, a formal recruitment protocol was developed with clinic staff during piloting (See Appendix 3) Small inducements in the form of a health products were used to increase recruitment.

Approval to examine patients for this study was granted by the joint University College London/ University College Hospital committee on the ethics of human research (Ref. No. 2498),

\subsubsection{Variables}

Independent variables in this study were:

i, demographic variables including age, occupation, race

ii, dental experience including attendance behaviour, oral hygiene behaviour, symptoms of periodontal diseases, previous dental treatment

iii, inhaled drug use (tobacco, opiates, amyl nitrate, cocaine, amphetamines, cannabis)

iv, oral manifestations of HIV infection

$\mathrm{v}, \quad$ teeth present

vi, plaque score

vii, calculus

viii, HIV sero-status

ix, Haematological status (CD4, CD8, total lymphocyte and neutrophil counts)

$\mathrm{x}, \quad$ CDC classification

$\mathrm{xi}$, Current and recent medications.

Outcome variables were:

i, Symptoms of periodontal diseases

ii, gingival health

iii, periodontal attachment

iv, foetor oris 


\subsubsection{Selection and development of measures}

A self-complete questionnaire was developed and piloted which enquired into social and recreational factors which might be related to periodontal health (Appendix 4). Reasons for attending the clinic on the day of the examination were investigated to identify selection bias.

\subsubsection{Demographic variables}

Conventional periodontitis is associated with age and occupation. Age was recorded in years and occupation was grouped by the Registrar-General's classification of socioeconomic status (OPCS, 1991). Race was also recorded.

\subsubsection{Inhaled drug use}

The effect of tobacco use in the aetiology of periodontal diseases has been discussed. There is little data available on the periodontal effects of other inhaled drugs but drug use is known to be common among homosexual men (Lemp et al, 1994) and both alcohol and drug use have been associated with unsafe sex in homosexual men (Nadeau et al, 1993). In one of the first reports of oral manifestations of HIV infection among homosexual men in San Francisco, 50\% smoked tobacco, 62\% smoked marijuana, 29\% used cocaine and 23\% used nitrite inhalants (Silverman et al, 1986).

Self report has been shown to be a valid measure of smoking behaviour (Pettiti et al, 1981; Pierce et al, 1987). Tobacco use was recorded as the form of tobacco used and the quantity used each day. Use of other inhaled drugs was recorded as ever/never and at present or not.

\subsubsection{4 $\quad \underline{\text { Stress }}$}

Whilst stress is implicated in the aetiology of periodontal diseases, the difficulties of measuring stress and the chance that its effects might be obscured by other variables precluded investigation of this variable in this study.

\subsubsection{5 $\quad$ Hand used to hold toothbrush}

Periodontal measures were made on a half mouth basis. The hand used to hold toothbrush was recorded to identify and exclude greater abrasion on the contra-lateral side (Todd and Lader, 1991). 
Data on symptoms of periodontal diseases were collected but are not reported here.

\subsubsection{Clinical measures}

\subsection{The presence of mucosal disease}

Oral manifestations of HIV infection were recorded using the presumptive diagnostic criteria of the USA Oral AIDS Collaborative Group (Greenspan et al, 1992).

\subsection{Teeth present}

To provide sufficient data only participants with 20 or more teeth were examined. Halfmouth examinations were performed as a compromise between the speed of partial scoring and the chance to identify specific teeth affected by periodontal diseases. The left and right sides of participants were examined alternatively unless there was gingival ulceration when all the teeth were examined. The side examined of the first participant of each day was decided by the toss of the coin. To expedite data collection, records were made of buccal sites only.

\subsection{Plaque}

The plaque index of Silness and Löe (1964) was selected as a precise and reliable index. The data from this index can also be summarised as the number of surfaces per individual with a given score. Plaque scores were recorded for the buccal and mesial surfaces of each tooth to consider oral hygiene facial and interdental surfaces. 


\subsection{Gingivitis}

Comprehensive descriptionaldata were zollected so that diagnoses could be made post hoc. A gingival index modified from Löe and Silness (1963) was used to record signs of gingival health at the mid-buccal and interdental areas on examined teeth.

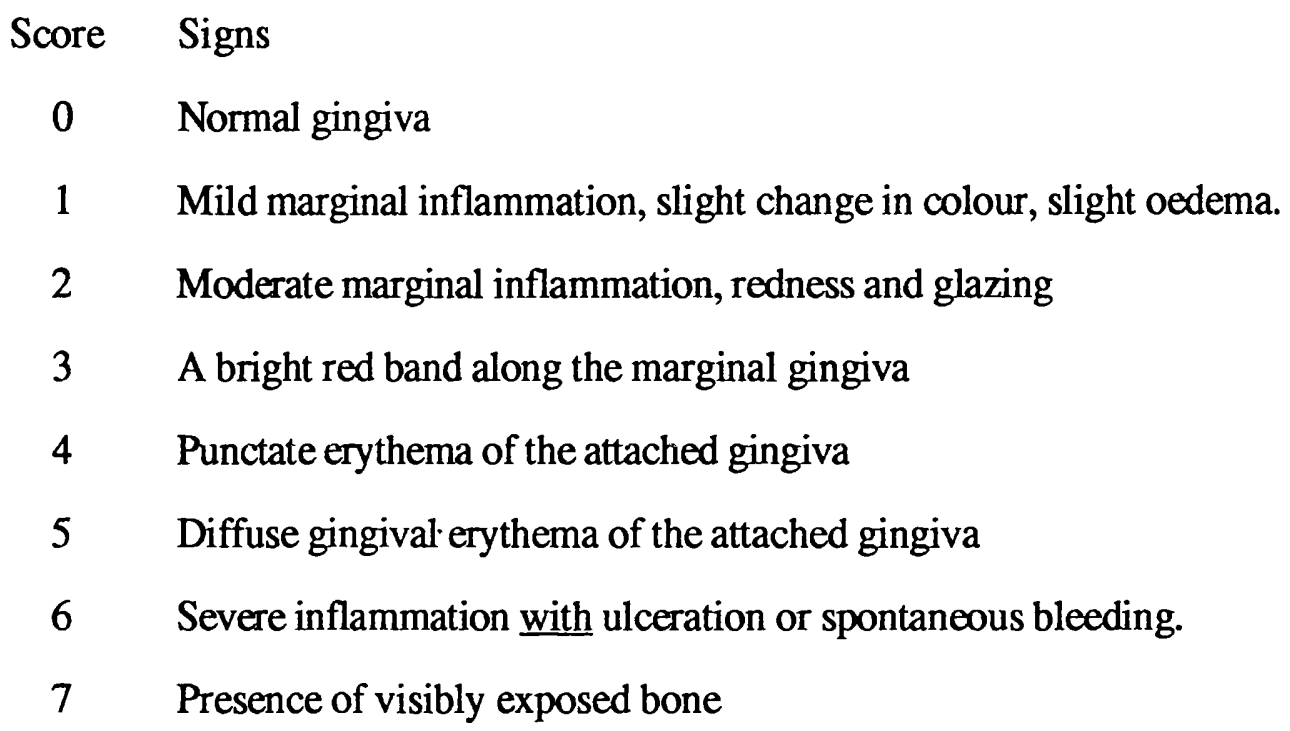

The presence or absence of each sign was recorded in four categories. A score of 0 excluded all other categories, scores 1 to 3 were mutually exclusive, 4 and 5 were mutually exclusive and 6 and 7 were additional categories.

Using this index the severity of conventional gingivitis was measured by scores of 0 to 2 . Bleeding on probing was recorded as present or absent for the three buccal sites on each tooth as a separate index. This was recorded on completion of probing each quadrant so that there was a consistent time between probing and recording of bleeding.

Gingival ulceration was described by the number of ulcers present, the number of teeth affected by the largest ulcer and the apical extent of the largest ulcer.

\subsection{Periodontitis}

The Ramfjord system of recording probing depths and attachment loss was selected since attachment loss in the absence of pocketing had been associated with HIV infection. Where the presence of a restoration or calculus prevented identification of the CEJ, measurements was made from the apical extent of the obstruction. 


\subsection{Mobility}

Mobility is a consequence of and directly related to attachment loss (Khoo and Watts, 1988) and was omitted as a duplication of attachment loss measurement.

\subsection{Foetor oris}

Foetor oris is not a consistent finding in ANUG. However, an attempt was made to record the presence of the characteristic foetor of ANUG despite the subjective nature of the measure.

\subsubsection{General Health}

Standardised data on general health were collected from participants' records (Appendix 5).

HIV-1 sero-status was recorded using the results from the standard ELISAs used at the clinic. The number of false positive test results is limited by the clinic protocol in which any positive assay is repeated. Participants were regarded as HIV infected if they had a confirmed positive test on the day of the examination or any time previously. Participants were considered negative if they had tested negative in the year before. Medical records of men who did not meet either criterion were reviewed again at the end of the period of data collection. Those who had a negative test after the examination were regarded as negative. All others were regarded as untested.

Clinical stage of HIV infection was recorded using the CDC revised classification (1992). CD4, CD8 and total lymphocyte counts are recorded every three months during HIV infection. Collection of these data allowed exploration of the best predictor of disease progression: either absolute $\mathrm{CD} 4$ count which is more precise; or $\mathrm{CD} 4 / \mathrm{CD} 8$ ratio which is more reliable (Fahey et al, 1990).

Neutrophil counts are made during HIV infection as part of haematological screening and were recorded to investigate any possible effects of neutropenia on periodontal health.

All haematological values used were recorded within 6 weeks of the examination except for a small number of men with AIDS who had not had CD4+ve lymphocyte counts for up to 8 months. In this case the most recent count was used (all were under 50 cells $/ \mathrm{mm}^{3}$ ). 
Medications used currently and in the last 3 months were recorded to identify mediating or interactive effects. Use of medications used in the management of HIV disease were recorded in closed items (specifically: co-trimoxazole, pentamidine, dapsone, nystatin, amphotericin, miconazole, ketoconazole, fluconazole, azidothymidine, ddI, ddC, acyclovir, gancyclovir, prednisolone or 'others' ). Current and recent use of medications less specific to HIV disease was recorded in open items. Medications first prescribed on the day of the examination were ignored.

\subsubsection{Data collection}

Records for all men booked into the clinic were viewed each morning. Those of men who may have been homosexual were flagged to remind clinic staff to suggest participation. Men interested in participation were introduced to a research assistant who explained the nature of the study and obtained written consent (Different information sheets and consent forms used for known HIV positive and other participants are enclosed in Appendices 6 - 9).

The questionnaire was given to all participants to complete and was finally used as a structured interview guide to ensure that it had been completed fully.

To allow the examiner to be blinded to the HIV status of participants, clinical examinations took place in a separate room and participants were asked not to reveal information about their status to the examiner. A single examiner was used throughout the study. Examinations were conducted with participants lying on a couch illuminated by a Daray portable halogen lamp held $60 \mathrm{~cm}$ away. Measurements were made with a Michigan probe (diameter $0.6 \mathrm{~mm}$ ) with Williams markings at a force of $0.25 \mathrm{~N}$

Examinations were conducted with the least invasive measures first in the following order:

i) presence of soft tissue lesions including gingival ulceration

ii) side of mouth to be examined (opposite side to previous participant)

iii) teeth present on side to be examined (all teeth if there was gingival ulceration)

iv) plaque scores

v) gingival index

vi) extent and severity of ulceration

vii) probing depths for upper quadrant

viii) bleeding on probing for upper quadrant before allowing participant to close

ix) probing depths for lower quadrant

x) bleeding on probing for the lower quadrant

xi) distances from the gingival margin to the CEJ 
xii) calculus score for each tooth

xii) presence or absence of foetor oris

Measurements were made starting on the upper teeth and always at the distal,

\subsubsection{1 $\quad$ Medical data}

Medical records were viewed one month after the examination to allow tests conducted on the day of the exam to be entered into the records.

\subsubsection{Data analysis.}

Summary measures were created to capture the presence, extent and severity of periodontal conditions without analysis of multiple non-independent observations using the individual as the unit for analysis (Imrey, 1986). A condition was defined as present if there were one or more sites with the condition. The extent of a condition was defined as the proportion of sites affected within each individual and severity was defined as the maximum score or index for the condition within each individual.

The data were skewed for six variables and were transformed to allow parametric analyses. Maximum probing attachment loss (PAL) was transformed to log. maximum PAL. Few men had probing depths or PAL of $4 \mathrm{~mm}$ or greater to allow analysis as continuous variables so dichotomous variables were created by whether men fell into the top quartile by the proportion of sites with probing depths or attachment loss greater than $4 \mathrm{~mm}$ (Sterne $e t$ $a l, 1990)$. The extent of gingivitis was converted into a categoric variable by the presence or absence of gingivitis at all teeth. The extent of bleeding on probing (BOP) was transformed into $\sqrt{ }$ (proportion of sites with BOP). CD4 counts were transformed to $\sqrt{(C D 4}$ counts). Data were normally distributed for all other variables and fulfilled the criteria for parametric and regression analyses.

Analysis was carried out in three phases. Initially, descriptive statistics and univariate analyses (chi square tests, student $t$ tests and Mann-Whitney Wilcoxon Tests) were used to describe and compare key descriptor variables and distributions of summary measures of periodontal health in men with and without HIV.

The second phase aimed to identify changes associated with HIV infection using multiple and logistic regression analyses as the primary strategies to control confounding (Rothman, 1986; Newman et al, 1988; Altman, 1991). Scatter plots were drawn for the presence of 
outliers in the data and assess possible linear relationships. Correlation, Student $t$ tests and linear regression were used in univariate analyses to assess the relationship between outcomes and the following independent variables: Age, HIV status, the proportion of surfaces with plaque, whether the participant held his toothbrush in the ipsi- or contralateral hand, race, socio-economic status, medications, alcohol (dichotomised around the median level of consumption) and the following recreational drugs (all recorded as ever or never), tobacco, cannabis, nitrite inhalants, amphetamines, cocaine, heroin and 'others'. Independent variables for which the null hypthesis had a probability of less than 0.2 were tried in forward stepwise regression models (JMP 2.0, SAS Institute Inc., Cary, NC, USA). Multiple regression was used for continuous outcome variables and logistic regression for dichotomous outcomes.

Only men with HIV infection were included in the final phase which aimed to identify possible mediators of the relationship between HIV and periodontal health. Independent variables specific to men with HIV or only available for men with HIV were introduced and tested in correlation and univariate analyses. These were: CD4+ve and CD8+ve and total lymphocyte counts; neutrophil counts; clinical staging of HIV disease; use of HIV related medications (dichotomised by whether each participant had taken the drug in the last 3 months) and the presence of the following oral manifestations of HIV: oral candidiasis; oral hairy leukoplakia; Kaposi's sarcoma; other oral manifestations and any oral manifestation. Associations with $P<0.2$, as well as significant variables in the second phase w ere tested in forward stepwise multiple and logistic regression models.

\subsubsection{Examiner reliability}

To assess the reproducibility of clinical measures examinations were repeated on 73 men (9.2\%) at the same visit.

The standard deviation of repeated measures of probing attachment loss (PAL) was 0.73 and when PAL was dichotomised ( $<4 \mathrm{~mm}$ or $\geq 4 \mathrm{~mm}$ ) kappa was 0.84 . Weighted kappa for plaque scores was 0.76 and as a dichotomous variable (plaque present/absent) kappa was 0.79. Weighted kappa for gingival index was 0.87 and kappa for BOP was 0.70. All of which were considered to be within acceptable levels. 


\subsubsection{Representativeness of the sample}

Two methods were chosen to assess the representativeness of the sample: firstly, to compare the number and characteristics of those recruited and not recruited during one month of the data collection and secondly, to evaluate the sample as a proportion of the total accessible population.

During December 1993 physicians were asked to account for each of their patients who met the inclusion criteria by recording whether: they had recruited the patient; the patient had already taken part in the project; they had forgotten to suggest participation; the patient had refused or any other outcome. 137 men from the accessible population visited the clinic on the days the examiner was present. Ninety four of these were or had already been examined (68.6\%). The remaining 43 were either not invited or refused to participate. Data on this group was drawn from the clinic database. They were found to be of similar socioeconomic status ( $P=0.32$, Chi sq.) but younger (mean 30.0 y vs. $34.0 \mathrm{y} . P=0.024$, Mann-Whitney-Wilcoxon test)

The clinic population is dynamic and the number of homosexual men with HIV attending is best estimated by the attendance for the six months in the middle of the data collection. Between January and June 1993, 638 homosexual men with HIV sought treatment at the clinic. In total, 312 men with HIV were examined during the study, thus examining on 2 days per week recruited approximately $49 \%$ of the accessible men with HIV. Comparable activity data are not recorded for patients without HIV.

\section{$\mathbf{5 . 4 \quad \text { Results }}$}

Complete data were collected for $794 \mathrm{men}$. Of these, 260 had tested negative for HIV in the year preceding or at any time since the examination and 312 were HIV antibody positive. The remaining 222 did not meet either criterion and were regarded as untested.

The men with HIV (Mean age, 35.9 years, 95\% central range 23.8-54.2y) were slightly older than those without (31.2 years, 95\% CR 19.5-57.5y, $P<0.001$, Mann-Whitney Wilcoxon Test) and fewer were students ( $2.2 \%$ v. $10.4 \%, P<0.001$, chi sq.). Both groups had similar numbers of teeth (Means: HIV+ve $=13.6 ; \mathrm{HIV}-\mathrm{ve}=13.8, P=0.229, \mathrm{MWWT}$ ) similar mean plaque scores $(\mathrm{HIV}+\mathrm{ve}=1.05, \mathrm{HIV}-\mathrm{ve}=1.00)$ and similar proportions of surfaces with detectable plaque (Means: HIV $+\mathrm{ve}=0.52(0.47-0.58,95 \%$ confidence interval); HIV-ve $=0.50(0.47-0.53,95 \% \mathrm{CI}), P=0.306, \mathrm{t}$ test $)$. The proportions of the total number of surfaces in the men with the different Löe and Silness plaque scores are presented in Appendix 10. 
Similar numbers of men with and without HIV had smoked tobacco (63.7\% overall), More men with HIV infection had used nitrite inhalants and there was a tendency for more infected men to have used cocaine and cannabis (Table 5.1),

Table 5.1. Frequency of inhaled drug use among 312 men with and 260 without HIV infection (dichotomised as ever or never)

\begin{tabular}{|l|cc|c|}
\hline \multicolumn{1}{|c|}{} & HIV+ve (\%) & HIV-ve (\%) & $\boldsymbol{P}$ (chi sq.) \\
\hline Tobacco & 65.1 & 62.1 & 0.491 \\
Cannabis & 60.3 & 53.1 & 0.084 \\
Nitrite inhalants & 70.2 & 58.1 & 0.002 \\
Amphetamines & 24.7 & 25.8 & 0.765 \\
Cocaine & 26.9 & 20.4 & 0.068 \\
Heroin & 4.2 & 5.4 & 0.490 \\
Other & 3.2 & 3.5 & 0.865 \\
\hline
\end{tabular}

More men with HIV visited the genito-urinary medicine clinic because of problems with their mouth or gums $(25(8 \%)$ and $9(3.5 \%)$ respectively. $P=0.042$, Chi sq.). Fourteen men (4.5\%) with HIV and $8(3.1 \%)$ without said they were visiting the clinic because of problems with their mouths and $4 \mathrm{HIV}+\mathrm{ve}$ men $(0.9 \%)$ were visiting because of gum problems compared to 1 HIV-ve man ( $0.4 \%)$. Seven men $(2.2 \%)$ with HIV attended for both gum and mouth problems whilst none of the HIV negative men did.

Seventy-one men with HIV (22.8\%) had a clinical indicator condition for AIDS. CD4 counts were available for $276 \mathrm{men}$, of whom 118 had counts less than $200 \mathrm{cells} / \mathrm{mm}^{3}$. The mean and median counts were 325 and 270 cells/ $/ \mathrm{mm}^{3}$ respectively. One hundred and sixtyeight men (53.8\%) met the 1993 case definition for AIDS.

One hundred and eighty-six men (59.6\%) with HIV had oral manifestations of the infection. The commonest of these were: OHL, 138 (44.2\%); erythematous candidiasis, 84 (26.9\%) and pseudomembranous candidiasis 36 (11.5\%). Seven men without HIV (2.6\%) had oral lesions which met the clinical criteria for oral manifestations of HIV.

The frequencies of periodontal changes in men with and without HIV are summarised in Table 5.2. More men with HIV had PAL, pocketing, diffuse and punctate erythema of the attached gingiva (all $P<0.001)$, gingivitis $(P<0.038)$ and BOP $(P<0.028)$. Bright red bands were equally common in men with and without HIV. 
Table 5.2. The presence of periodontal changes in homosexual men by HIV status

\begin{tabular}{|c|c|c|c|c|}
\hline & Summary measure & $\begin{array}{l}\text { HIV+ve } \\
\text { n (\%) }\end{array}$ & $\begin{array}{l}\text { HIV-ve } \\
\text { n (\%) }\end{array}$ & $\boldsymbol{P}$ (Chi sq.) \\
\hline Presence PAL & $1+$ site with PAL $\geq 4 \mathrm{~mm}$ & $186(59.6)$ & $74(28.5)$ & $<0.001$ \\
\hline Presence pocketing & $\begin{array}{l}1+\text { site with pocket } \geq \\
4 \mathrm{~mm}\end{array}$ & $159(51.0)$ & $83(31.9)$ & $<0.001$ \\
\hline Presence of gingivitis & $1+$ site with gingivitis & $309(97.9)$ & $251(96.5)$ & 0.038 \\
\hline Presence of BOP & 1+ site with BOP & $301(96.5)$ & $240(92.3)$ & 0.028 \\
\hline Presence of ulceration & $\begin{array}{l}1+\text { site with gingival } \\
\text { ulceration }\end{array}$ & $10(3.21)$ & $5(1.04)^{*}$ & 0.031 \\
\hline Presence of red band & $\begin{array}{l}1+\text { site with bright red } \\
\text { band }\end{array}$ & $38(12.2)$ & $26(10.0)$ & 0.410 \\
\hline $\begin{array}{l}\text { Presence of diffuse } \\
\text { erythema }\end{array}$ & $\begin{array}{l}1+\text { site with diffuse } \\
\text { erythema }\end{array}$ & $39(12.5)$ & $8(3.1)$ & $<0.001$ \\
\hline $\begin{array}{l}\text { Presence of punctate } \\
\text { erythema }\end{array}$ & $\begin{array}{l}1+\text { site with punctate } \\
\text { erythema }\end{array}$ & $30(9.6)$ & $3(1.1)$ & $<0.001$ \\
\hline
\end{tabular}

$\mathrm{PAL}=$ Probing attachment loss.

$\mathrm{BOP}=$ Bleeding on probing.

* Men not tested for HIV were regarded as HIV-ve to increase the power of the analysis for gingival ulceration only. Includes 1 man with ulceration not tested for HIV.

PAL and pocketing were more severe and extensive in men with HIV. Mean maximum PAL in men with and without HIV was $4.33 \mathrm{~mm}$ and $2.98 \mathrm{~mm}$ respectively (95\% CRs 1.810.0 and $1.0-7.0$ respectively, $P<0.001, \mathrm{t}$ test using the $\log$ transformation) and mean maximum pocket depth was $3.73 \mathrm{~mm}$ and $3.37 \mathrm{~mm}$ respectively (95\% CIs 3.51-3.86 and 3.23-3.41 respectively, $P<0.001, t$ test). The median proportions of sites with PAL in the two groups were 0.024 and 0.000 respectively (95\% CRs $0.000-0.441$ and $0.000-0.213$, $P<0.001$. MWWT) and the median proportions of sites with pocketing were 0.021 and 0.000 respectively (95\% CRs $0.000-0.232$ and $0.000-0.167, P<0.001$. MWWT). The mean proportions of sites with pockets were 0.036 and 0.019 respectively.

Gingival ulceration, diffuse and punctate erythema of the attached gingiva and red banding were uncommon and the samples were too small to justify analysis of the extent of these variables. Gingival ulceration was present in only 15 men ( $10 \mathrm{HIV}+\mathrm{ve}, 4 \mathrm{HIV}$-ve and 1 not tested) thus samples were too small to detect differences in prevalence of the condition. Statistical power was increased by regarding 222 men not tested for HIV as HIV-ve, conferring a relative risk for gingival ulceration in HIV infection of 3.090 with a $95 \%$ confidence interval of 0.93 to 8.99. Of the 10 men with HIV and gingival ulceration, 6 had lesions which extended less than $4 \mathrm{~mm}$ from the CEJ, the remaining 4 extended $4 \mathrm{~mm}$ or more. Bone was neither visible nor palpable in any men. All 15 men with gingival 
ulceration had the characteristic foetor oris of acute necrotising ulcerative gingivitis. Men without gingival ulceration did not have foetor.

Of the men with HIV, 199 (63.8\%) had a maximum conventional gingivitis score of 2 compared to 120 (46.1\%) without HIV ( $P<0.001$. Chi sq.). The mean proportions of sites with gingivitis among men with and without HIV were 0.777 and 0.616 respectively $(95 \%$ CRs 0.000-1.000 and 0.138-1.000, $P<0.001$ MWWT). One hundred and twenty-five men (40.1\%) with HIV had gingivitis at all teeth compared to $46(17.7 \%)$ men without $(P<0.001$, Chi sq.).

Table 5.3 presents the best regression models for outcomes related to loss of periodontal attachment in the men of known HIV status. More surfaces with plaque, HIV infection and increased age were consistently associated with all summary measures of PAL and pocketing. Tobacco use was strongly and consistently related to PAL but not to pocketing. An apparent association between history of cocaine use and the severity of PAL was excluded in multiple regression.

Regressors for gingival outcomes are summarised in Table 5.4. HIV infection was strongly associated with the extent and severity of gingivitis and there was a strong tendency for HIV to be associated with the presence of gingivitis. HIV infection was also associated with the presence of bleeding on probing, gingival ulceration, diffuse and punctate erythema. Two independent variables tested in the multiple and logistic regression models were not significantly associated with gingival outcomes. These were: the use of non-HIV related medication for the extent of bleeding on probing and history of cocaine use for the presence of red bands.

Only men with HIV were included in the third phase of analysis. Table 5.5 summarises the best regression models for summary measures of PAL and pocketing and indicates a strong inverse relationship between CD4 counts and the presence and extent of periodontal attachment loss. There was a similar trend $(P=0.06)$ for decreased CD4 counts to be associated with the severity of attachment loss. 
Table 5.3. Best forward stepwise multiple and logistic regression models for outcomes related to loss of periodontal attachment in 312 men with and 260 without HIV infection.

\begin{tabular}{|c|c|c|c|c|c|c|}
\hline Outcome & $\begin{array}{l}\quad \begin{array}{l}\text { Age } \\
b \\
P\end{array} \text { (se) } \\
\end{array}$ & $\begin{array}{l}\text { HIV status } \\
b \text { (se) } \\
P\end{array}$ & $\begin{array}{l}\text { Tobacco use } \\
b \text { (se) } \\
P\end{array}$ & $\begin{array}{l}\quad \text { Plaque } \\
b \text { (se) } \\
P\end{array}$ & $S D_{\text {res }}$ & $R^{2}$ \\
\hline $\begin{array}{l}\text { Presence } \\
\text { PAL }\end{array}$ & $\begin{array}{l}0.078(0.049) \\
<0.001\end{array}$ & $\begin{array}{l}0.526(0.096) \\
<0.001\end{array}$ & $\begin{array}{l}0.383(0.099) \\
<0.001\end{array}$ & $\begin{array}{l}1.007(0.393) \\
0.010\end{array}$ & & 0.149 \\
\hline Extent PAL ${ }^{\mathrm{L}}$ & $\begin{array}{l}0.078(0.013) \\
<0.001\end{array}$ & $\begin{array}{l}0.581(0.120) \\
<0.001\end{array}$ & $\begin{array}{l}0.414(0.118) \\
<0.001\end{array}$ & $\begin{array}{l}1.380(0.449) \\
0.002\end{array}$ & & 0.158 \\
\hline Severity PAL & $\begin{array}{l}0.069(0.006) \\
<0.001\end{array}$ & $\begin{array}{l}0.490(0.078) \\
<0.001\end{array}$ & $\begin{array}{l}0.368(0.078) \\
<0.001\end{array}$ & $\begin{array}{l}1.118(0.311) \\
<0.001\end{array}$ & 1.791 & 0.228 \\
\hline $\begin{array}{l}\text { Presence } \\
\text { Pocketing } \mathrm{L}\end{array}$ & $\begin{array}{l}0.025(0.011) \\
0.021\end{array}$ & $\begin{array}{l}0.339(0.091) \\
<0.001\end{array}$ & & $\begin{array}{l}0.905(0.364) \\
0.013\end{array}$ & & 0.043 \\
\hline $\begin{array}{l}\text { Extent } \\
\text { PocketingL }\end{array}$ & $\begin{array}{l}0.028(0.012) \\
0.018\end{array}$ & $\begin{array}{l}0.368(0.109) \\
<0.001\end{array}$ & & $\begin{array}{l}1.718(0.428) \\
0.001\end{array}$ & & 0.064 \\
\hline $\begin{array}{l}\text { Severity } \\
\text { Pocketing }\end{array}$ & $\begin{array}{l}0.014(0.006) \\
0.016\end{array}$ & $\begin{array}{l}0.142(0.049) \\
0.004\end{array}$ & & $\begin{array}{l}0.620(0.194) \\
0.001\end{array}$ & 1.121 & 0.051 \\
\hline
\end{tabular}

PAL $=$ Probing attachment loss.

$b=$ regression coefficient

se $=$ standard error

$\mathrm{SD}_{\text {res }}=$ residual standard deviation

$R^{2}=$ proportion of variation explained by the model

$\mathrm{L}=$ Logistic regression analysis (other analyses are multiple regression) 
Table 5.4. Best forward stepwise multiple and logistic regression models for gingival health outcomes in 312 men with and 260 without HIV infection?.

\begin{tabular}{|c|c|c|c|c|c|}
\hline Outcome & $\begin{array}{l}\text { HIV status } \\
b \text { (se) } \\
P\end{array}$ & $\begin{array}{l}\text { Tobacco use } \\
b \text { (se) } \\
P\end{array}$ & $\begin{array}{l}\text { Plaque } \\
b \text { (se) } \\
P\end{array}$ & SD $_{\text {res }}$ & $R^{2}$ \\
\hline $\begin{array}{l}\text { Presence of } \\
\text { gingivitis } L\end{array}$ & $\begin{array}{l}0.673(0.370) \\
0.069\end{array}$ & $\begin{array}{l}0.728(0.337) \\
0.031\end{array}$ & $\begin{array}{l}11.569(2.763) \\
<0.001\end{array}$ & & 0.370 \\
\hline $\begin{array}{l}\text { Extent of } \\
\text { gingivitis }{ }^{L *}\end{array}$ & $\begin{array}{l}0.680(0.115) \\
<0.001\end{array}$ & & $\begin{array}{l}4.862(0.516) \\
<0.001\end{array}$ & & 0.225 \\
\hline $\begin{array}{l}\text { Severity of } \\
\text { gingivitis } L\end{array}$ & $\begin{array}{l}0.378(0.092) \\
<0.001\end{array}$ & & $\begin{array}{l}3.439(0.422) \\
<0.001\end{array}$ & & 0.123 \\
\hline Presence of BOPL & $\begin{array}{l}0.388(0.197) \\
0.049\end{array}$ & & $\begin{array}{l}4.400(0.996) \\
<0.001\end{array}$ & & 0.122 \\
\hline Extent of $\mathrm{BOP}^{\dagger}$ & & & $\begin{array}{l}0.473(0.035) \\
<0.001\end{array}$ & 0.200 & 0.253 \\
\hline $\begin{array}{l}\text { Presence of } \\
\text { Ulceration } \mathrm{L} \uparrow\end{array}$ & $\begin{array}{l}0.698(0.280) \\
0.013\end{array}$ & & & & 0.065 \\
\hline $\begin{array}{l}\text { Presence of red } \\
\text { band }^{L}\end{array}$ & - & & $\begin{array}{l}3.386(0.615) \\
<0.001\end{array}$ & & 0.098 \\
\hline $\begin{array}{l}\text { Presence of } \\
\text { diffuse erythema } \mathrm{L}\end{array}$ & $\begin{array}{l}0.744(0.200) \\
<0.001\end{array}$ & & $\begin{array}{l}1.832(0.655) \\
0.005\end{array}$ & & 0.081 \\
\hline $\begin{array}{l}\text { Presence of } \\
\text { punctate erythema } L \S\end{array}$ & $\begin{array}{l}1.054(0.307) \\
<0.001\end{array}$ & & & & 0.115 \\
\hline
\end{tabular}

$\mathrm{BOP}=$ Bleeding on probing

$b=$ regression coefficient

se $=$ standard error

$\mathrm{SD}_{\text {res }}=$ residual standard deviation

$R^{2}=$ proportion of variation explained by the model

$\mathrm{L}=$ Logistic regression analysis (other analyses are multiple regression)

* History of 'other drug' use was significant in the model for the extent of gingivitis. $b=$ 0.618 , se $=0.287 \& P=0.031$.

$\dagger$ History of nitrite inhalant use was significant in the model for the extent of BOP. $b=$ 0.740 , se $=0.009 \& P=0.006$.

I Gingival ulceration was present in only 15 men and the power of the analysis was increased by regarding men not tested for HIV as HIV-ve.

$\S$ History of nitrite inhalant use was significant in the model for the presence of punctate erythema. $b=0.632$, se $=0.273 \& P=0.021$. 
Table 5.5. Best forward stepwise multiple and logistic regression models for outcomes related to loss of periodontal attachment in 312 men with HIV infection.

\begin{tabular}{|c|c|c|c|c|c|c|}
\hline Outcome & $\begin{array}{l}\quad \text { Age } \\
b \text { (se) } \\
P\end{array}$ & $\begin{array}{l}\quad \sqrt{\text { CD4 }} \\
b(\mathrm{se}) \\
P\end{array}$ & $\begin{array}{l}\text { Tobacco use } \\
b \text { (se) } \\
P\end{array}$ & \begin{tabular}{l}
\multicolumn{1}{c}{ Plaque } \\
$b$ (se) \\
$P$
\end{tabular} & SD $_{\text {res }}$ & $R^{2}$ \\
\hline Presence PAL ${ }^{\mathrm{L}}$ & $\begin{array}{l}0.059(0.018) \\
0.001\end{array}$ & $\begin{array}{l}-1.327(0.584) \\
0.023\end{array}$ & $\begin{array}{l}0.530(0.141) \\
<0.001\end{array}$ & $\begin{array}{l}1.361(0.574) \\
0.018\end{array}$ & & 0.091 \\
\hline Extent PAL ${ }^{\mathrm{L}}$ & $\begin{array}{l}0.055(0.012) \\
0.002\end{array}$ & $\begin{array}{l}-0.250(0.132) \\
0.027\end{array}$ & $\begin{array}{l}0.503(0.151) \\
<0.001\end{array}$ & $\begin{array}{l}2.201(0.590) \\
<0.001\end{array}$ & & 0.112 \\
\hline Severity PAL* & $\begin{array}{l}0.014(0.004) \\
<0.001\end{array}$ & $\begin{array}{l}-0.250(0.132) \\
0.060\end{array}$ & $\begin{array}{l}0.122(0.030) \\
0.001\end{array}$ & $\begin{array}{l}0.310(0.117) \\
0.009\end{array}$ & 0.462 & 0.186 \\
\hline $\begin{array}{l}\text { Presence } \\
\text { Pocketing } 4\end{array}$ & & & & $\begin{array}{l}1.714(0.523) \\
0.001\end{array}$ & & 0.048 \\
\hline $\begin{array}{l}\text { Extent } \\
\text { Pocketing } \mathrm{L}\end{array}$ & & & & $\begin{array}{l}1.888(0.538) \\
<0.001\end{array}$ & & 0.045 \\
\hline $\begin{array}{l}\text { Severity } \\
\text { Pocketing } \dot{\dagger}\end{array}$ & & & & $\begin{array}{l}1.034(0.267) \\
<0.001\end{array}$ & 1.089 & 0.098 \\
\hline
\end{tabular}

$\mathrm{PAL}=$ Probing attachment loss.

$b=$ regression coefficient

$\mathrm{se}=$ standard error

$\mathrm{SD}_{\mathrm{res}}=$ residual standard deviation

$R^{2}=$ proportion of variation explained by the model

$\mathrm{L}=$ Logistic regression analysis (other analyses are multiple regression)

* 3 other variables were associated with the severity of PAL in the model. These were: history of Dapsone use, $b=-0.192$, se $=0.085 \& P=0.024$; history of ddI use, $b=0.158$, se $=0.081 \& P=0.053$ and the presence of oral candidiasis, $b=-0.061$, se $=0.031 \& P=$ 0.047 .

$\{2$ other variables were associated with the presence of pocketing: Total lymphocyte count, $b==0.310$, se $=0.155 \& P=0.045$ and the presence of mucosal ulceration, $b=0.534$, se $=0.271 \& P=0.049$.

$\dagger 2$ other variables were associated with the severity of pocketing: Total lymphocyte count, $b=0.315$, se $=0.081 \& P=<0.005$ and the presence of hairy leukoplakia, $b=-0.139$, se $=$ $0.064 \& P=0.032$. 
Table 5.6. Best forward stepwise multiple and logistic regression models for gingival health outcomes in 312 men with HIV infection.

\begin{tabular}{|c|c|c|c|c|c|}
\hline Outcome & $\begin{array}{l}b \text { (se) }^{\sqrt{\mathrm{CD4}}} \\
P\end{array}$ & $\begin{array}{l}\text { Oral candida } \\
b \text { (se) } \\
P\end{array}$ & $\begin{array}{l}\text { Plaque } \\
b \text { (se) } \\
P\end{array}$ & SDres & $R^{2}$ \\
\hline $\begin{array}{l}\text { Extent of } \\
\text { gingivitis }{ }^{L *}\end{array}$ & & $\begin{array}{l}0.304(0.146) \\
0.037\end{array}$ & $\begin{array}{l}4.849(0.698) \\
<0.001\end{array}$ & & 0.213 \\
\hline $\begin{array}{l}\text { Severity of } \\
\text { gingivitis }\end{array}$ & & $\begin{array}{l}0.357(0.140) \\
0.011\end{array}$ & $\begin{array}{l}3.718(0.613) \\
<0.001\end{array}$ & & 0.133 \\
\hline Extent of BOP & $\begin{array}{l}0.190(0.051) \\
<0.001\end{array}$ & & $\begin{array}{l}0.409(0.049) \\
<0.001\end{array}$ & 0.195 & 0.222 \\
\hline $\begin{array}{l}\text { Presence of red } \\
\text { band } L\end{array}$ & & & $\begin{array}{l}3.431(0.801) \\
<0.001\end{array}$ & & 0.089 \\
\hline $\begin{array}{l}\text { Presence of } \\
\text { diffuse erythema } L\end{array}$ & & $\begin{array}{l}0.560(0.178) \\
0.002\end{array}$ & $\begin{array}{l}1.987(0.767) \\
0.010\end{array}$ & & 0.077 \\
\hline $\begin{array}{l}\text { Presence of } \\
\text { punctate } \\
\text { erythema } \mathrm{L} \text {. }\end{array}$ & . & $\begin{array}{l}0.893(0.219) \\
<0.001\end{array}$ & & & 0.144 \\
\hline
\end{tabular}

$\mathrm{BOP}=$ Bleeding on probing

$b=$ regression coefficient

se $=$ standard error

$\mathrm{SD}_{\text {res }}=$ residual standard deviation

$R^{2}=$ proportion of variation explained by the model

$\mathrm{L}=$ Logistic regression analysis (other analyses are multiple regression)

* Neutrophil count was significant in the model for the extent of gingivitis, $b=0.241$, se $=$ 0.103 and $P=0.019$. 
Marginal gingivitis and bleeding on probing were so ubiquitous among men with HIV that regression analysis was inappropriate for associations with their presence. The best regression models for other gingival outcomes in men with HIV are presented in Table 5.6 The number of surfaces with plaque was strongly and consistently associated with summary measures of marginal gingivitis, bleeding on probing, the presence of a bright red band and the presence of diffuse erythema. Oral candidiasis was associated with measures of marginal gingivitis and with the diffuse and punctate erythema of the attached gingiva. The bright red band was only associated with the proportion of surfaces with plaque. An association between history of acyclovir use and the extent of gingivitis apparent in univariate analysis was not significant in the regression model.

Some of this data can be presented in stratified analyses which allows intuitive interpretation but can control fewer variables (Rothman, 1986). The prevalence of attachment loss is consistently greater in HIV infection when stratified by tobacco use and the severity of attachment loss is greater in men with HIV independent of the proportions of surfaces with plaque (Appendices 11 and 12). In contrast, Appendix 13 shows differences in the relationship between age and the extent of pocketing when stratified by HIV infection and Appendix 14 shows the different relationships between the prevalence of attachment loss and pocketing by CD4 count. Appendix 15 depicts the severity of conventional gingivitis and the presence of punctate erythema of the attached gingiva in men with and without HIV stratified by the presence of oral candidiasis.

\section{$5.5 \quad$ Discussion}

These data provide strong evidence that a number of periodontal changes are associated with HIV infection. These were: the presence, extent and severity of probing attachment loss, pocketing and conventional forms of gingivitis. Gingival ulceration, bleeding on probing and diffuse and punctate erythema of the attached gingiva were also more common in HIV disease. The regression analyses indicate that the association between HIV and these outcomes is consistent and independent of other factors implicated in their pathogenesis (most notably age, plaque and tobacco use)(Tables 5.3 and 5.4). Methodological differences, particularly sample size, control of other variables and reduced selection bias may account for the variation between these findings and those of other research.

The presence, extent and severity of both probing attachment loss and pocketing were increased in HIV infection. High levels of chronic periodontitis have been found in uncontrolled cross-sectional studies of people with HIV (Masouredis et al, 1992; Riley $e t$ 
$a l, 1992)$ and in longitudinal studies (Barr et al, 1992; Yeung et al, 1993) but this is the first study to find differences in standard measures of periodontal attachment loss between HIV positive and negative people.

In the regression analysis all three measures of attachment loss increased with lower CD4 lymphocyte counts whereas pocketing was not related to CD4 counts (Table 5.5). In a similar study, Grbic et al. (1995) detected neither a difference in the extent of pocketing between homosexual men with and without HIV nor more extensive pocketing in homosexual men with CD4 counts below 200 cells $/ \mathrm{mm}^{3}$. The present study is larger than that by Grbic et al. (four groups were studied: homosexual men with and without HIV and injecting drug users (IDUs) with and without HIV (77, 44, 44 and 39 men respectively). Pocketing was considerably more extensive in all participants (mean proportions of sites with pocketing were 0.242 and 0.214 in HIV positive and negative men compared to 0.036 and 0.019 in this study). Grbic et al. have not presented data on attachment loss or the prevalence or severity of pocketing. A recent study of 101 dental patients with HIV (84\% drug users) also failed to associate severity of periodontal disease (sic) and CD4 counts (Martínez-Canut et al, 1996). Complete data were available only for 64 patients and no account was taken of other variables than age.

In the present study, increased attachment loss may be the legacy of past episodes of ulceration which has destroyed periodontal tissue causing attachment loss without pocketing. If people with HIV have recurrent episodes of ulceration it is to be expected that the prevalence, extent and severity of such defects will increase over time as HIV disease progresses. However, recession is a feature of advanced destructive disease in older people in conventional populations (Yoneyama et al, 1988). The dramatic increases in pocketing may to some extent be ecological fallacy. Even among men without HIV, mean maximum pocket depth was $3.37 \mathrm{~mm}$, thus small increases in pocket depth, possibly due to false pocketing, would have a dramatic effects on the summary measures for extent and severity of pockets.

False pocketing may have been due to gingivitis which was more prevalent, extensive and severe in HIV infection (Tables 5.2 and 5.4). However, gingivitis was not related to progression of HIV disease as measured by CD4 lymphocyte counts but was strongly associated with the presence of plaque and oral candidiasis (Table 5.6). Oral candidiasis was also strongly associated with the presence of punctate and diffuse erythema of the attached gingiva. Candida species are uncommon in the sub-gingival micro flora of general populations but have been detected in plaque (Murray et al, 1989; Zambon et al, 1990) and invading the gingival epithelium of people with HIV (Odden et al, 1994). 
Grbic et al. (1995) also reported an association between oral candidiasis and linear gingival erythema but did not report on erythema of the attached gingiva. LGE was strongly related to plaque levels in both in both HIV+ve and negative homosexual men and IDUs (16.6\% Vs $11.4 \%$ and $38.5 \%$ Vs. $29.5 \%$ both $P>0.05$, Chi sq.) but was significantly associated with oral candidiasis independent of CD4 counts among the HIV+ve homosexual men in analysis of variance. This latter relationship was not seen in the IDUs and the authors suggested that any association may have been obscured by the poor oral hygiene in this group. However, there is a possible flaw in their analysis. The prevalence of LGE was similar in homosexual men with and without HIV, yet the LGE in the men with HIV was associated with oral candidiasis. By implication there must have been less non-candida related LGE in the men with HIV.

Bleeding on probing was more common in HIV infection in both univariate and regression analysis although no relationship was detected between the extent of bleeding and the infection. Among infected men, bleeding was so ubiquitous (96.5\%) that regression analysis was inappropriate (This figure is almost identical to that found by Swango (1991)). However, analysis of the men with HIV found bleeding on probing was less extensive with advanced HIV disease although the extent of bleeding on probing was not related to CD4 counts in the smaller study by Grbic et al. (1995). One explanation might be the hypovolaemia and changes in tissue vascularity of cachexia seen in late HIV disease which might cause less bleeding.

Gingival ulceration was associated with HIV infection in both chi square and regression analysis and the relative risk was 3.09 compared to other homosexual men. The $95 \%$ confidence interval for the relative risk ( 0.93 to 8.99) just includes 1 which reflects the poor statistical power for an uncommon outcome. Selection bias may have caused an over estimate of the relative risk and although compelling, these data are not conclusive evidence of an association between gingival ulceration and HIV. Three per cent of men with HIV had gingival ulceration. Allowing for selection bias this figure is comparable to findings in other studies. The annual incidence of gingival ulceration or NUP among patients at dedicated clinics has been reported at between 1 and 2\% (Glick et al, 1994; Robinson and Zakrzewska, 1994) and $1 \%$ in a non-self selected study population reported by Riley $e t$ al. (1992). Two other studies of non-self-selected groups have reported gingival ulceration and/or cratering in 4-6\% and 25\% of people with HIV (Swango et al, 1991; Masouredis et $a l, 1992)$. Different diagnostic criteria may account for the differences. For example, up to $20 \%$ of the participants diagnosed with HIV-P by Masouredis et al. (1992) did not have gingival ulceration. 
Six of ten men with gingival ulcers had ulcers which extended up to $3 \mathrm{~mm}$ from the cemento-enamel junction. Three millimetres was chosen as a criterion to objectively reflect the EC-Clearinghouse (1993) criterion for NUG in which ulceration is limited to the inter dental papilla. Using this translation $6(1.9 \%)$ men with HIV had necrotising ulcerative gingivitis and $4(1.3 \%)$ had necrotising ulcerative periodontitis. None had necrotising stomatitis.

A bright red band of the marginal gingiva has long been associated with HIV infection (Winkler et al, 1986) but the prevalence varies between 0 and $50 \%$ in different studies (Friedman et al, 1991; Masouredis et al, 1992). In the present study the prevalence in the study and control groups was 12.2 and $10.0 \%$ respectively, prevalences similar to those found among 200 people with HIV found by Riley et al. (1992). Higher prevalences were detected in two uncontrolled studies. Masouredis et al. (1992) added the prevalence of red banding, diffuse and punctate erythema to create an aggregate prevalence of $31 \%$ to $50 \%$ depending on the criteria used: Forty-nine per cent of 189 HIV positive people described by Swango et al. (1991) had 'a continuous erythematous band at least $1 \mathrm{~mm}$ in width' and it is possible that at least some of the erythema may have been chronic marginal gingivitis. Other smaller studies have not found atypical gingivitis in HIV infection (Friedman et al, 1991; Winkler et al, 1992). In the present study, bright red bands of the marginal gingiva were strongly associated with the presence of plaque, independent of HIV status. Red bands first described by Winkler et al. (1987) and now called linear gingival erythema may be so uncommon that a sample of 312 is too small to detect any cases.

The findings of this study must be viewed with care for several reasons. Firstly, crosssectional studies of this type are useful to identify associations between variables but do not imply cause and effect. Longitudinal and experimental studies are required to further investigate these findings.

More men with HIV were visiting the clinic with gingival problems than men without HIV (3.1\% \& $0.4 \%$ respectively), thus some selection bias is present. The examinations were conducted at the genito-urinary medicine clinic to reduce bias but some people with HIV seek care from their physicians for oral problems. Selection bias may therefore have caused an overestimate of the prevalence and relative risk of acute gingival disease (especially gingival ulceration) associated with HIV infection. It is unlikely that this bias accounts for the difference in the more common chronic problems.

Sixty per cent of the men with HIV had oral manifestations of the disease. This inevitably led to a 'partial-sightedness' of the examiner and may have caused some measurement bias. 
It is difficult to see how this problem might be avoided. Excluding people with oral manifestations would select less immuno-suppressed subjects, would be less likely to detect differences between groups and would prevent detection of the relationships between oral candidiasis and gingivitis. At first glance, these very relationships support concerns about measurement bias but the lack of association between periodontal outcomes and OHL (the most common oral manifestation) suggests this was not a significant source of bias.

Many men in this study had advanced HIV infection. Fifty-four per cent met the 1993 case definition for AIDS and they would be the most likely to manifest periodontal changes associated with the disease. The prevalence, extent and severity of periodontal changes would be lower in populations with early HIV disease. However, the range of disease in this study lent greater power to the regression analyses to associate a range of periodontal changes with HIV infection and identify potential factors in their aetiology.

\section{$5.6 \quad$ Conclusions}

Several periodontal changes are associated with HIV infection. These are: the presence, extent and severity of probing attachment loss, pocketing and conventional forms of gingivitis, gingival ulceration, bleeding on probing and diffuse and punctate erythema of the attached gingiva. These relationships are independent of other aetiological factors (particularly age, plaque and tobacco use).

Bright red bands were not associated with HIV infection but were associated with high plaque levels. 'HIV-associated gingivitis' or 'Linear gingival erythema' may be uncommon.

The prevalence, extent and severity of periodontal attachment loss increase with more advanced HIV disease. Although greater than in non-HIV infected men, the prevalence, extent and severity of periodontal pocketing do not increase in advanced HIV disease.

Oral candidiasis, and not the progression of HIV disease is a risk factor for the extent and severity of conventional gingivitis and erythema of the attached gingiva.

Gingival ulceration is approximately 3 times more common in HIV infection than in men of known negative or indeterminate status. The prevalence of necrotising gingivitis and necrotising periodontitis were less than $1.9 \%$ and $1.3 \%$ respectively.

Methodological differences may account for the variation between these findings and those of other research. 


\section{CHAPTER 6 GINGIVAL ULCERATION IN HIV INFECTION:} A CASE SERIES AND CASE CONTROL STUDY

\subsection{Abstract}

\section{Objectives}

Describe HIV-associated gingival ulceration, identify risk factors for incidence, extent, severity and relapse.

\section{Design}

Case series and case controlled study.

\section{Method}

Review of all cases seen at a dedicated dental clinic over a 5 year period and compare with controls.

\section{Results}

Ninety-four (7.1\%) of 1308 patients seen had 146 episodes of gingival ulceration. The cases fell into two distinct groups. The first group consisted of 89 patients who had 140 episodes of gingival ulceration similar to acute necrotising ulcerative gingivitis (ANUG) and responded well to conventional treatment for ANUG. One third of affected patients had a relapse. The cases were compared with 269 controls. Using forward stepwise logistic regression analysis, gingival ulceration was associated with oral candidiasis, lower age and lack of a diagnosis of AIDS. Among patients with AIDS co-trimoxazole use was inversely associated with gingival ulceration. When data were stratified by co-trimoxazole use the apparent relationship between ulceration and lack of AIDS diagnosis disappeared.

The second group were 5 patients who were neutropenic and had extensive ulceration without the typical microflora of ANUG. Histopathological examination, viral and bacterial culture revealed non-specific changes. The ulcers did not respond to the treatment regimen for ANUG but all 5 responded to treatment of their neutropenia.

\section{Conclusions}

Gingival ulceration is not common in HIV infection. Most cases resemble severe ANUG. Relapse of this disease is common. It is more frequent in younger people, those with oral candidiasis and without AIDS. Co-trimoxazole may be protective. A minority of cases with ulceration and associated neutropenia resembled the non-specific oral ulceration associated with HIV. 


\subsection{Introduction}

Acute necrotising ulcerative gingivitis (ANUG) has been reported in homosexual men not tested for HIV as well as people known to be HIV infected (Dennison et al, 1985; Pindborg et al, 1986; Pindborg and Holmstrup , 1987).

An ANUG-like lesion, characterised by severe pain, marked localised soft tissue necrosis, ulceration and inter proximal cratering was also described by Winkler et al. (1986). They named this disease AIDS Virus Associated Periodontitis or HIV-Periodontitis (Winkler $e t$ al, 1986; Winkler et al, 1988). Lesions did not have deep pockets but there was loss of crestal bone coincident with soft tissue destruction. In some cases bone was exposed and subsequently sequestrated (Winkler et al, 1988). Winkler \& Murray (1987) distinguished HIV-P from ANUG by its rapid progression. They believed ANUG could only cause bone loss after a number of years compared to $90 \%$ attachment loss documented over a six month period in one of their own patients. Murray et al. (1988) investigated the subgingival plaque of a small number of sites with HIV-P and found the levels of spirochaetes present in the lesions to be variable and not consistent with the microflora associated with ANUG.

A similar disease, HIV-necrotising gingivitis, was associated with HIV by the European Clearinghouse on oral problems related to HIV infection (1991). A subsequent conference renamed it 'necrotising (ulcerative) gingivitis' (NUG)(EC-Clearinghouse, 1993). The presumptive diagnostic criterion for NUG was 'Destruction of one or more interdental papillae. In the acute stage of the process ulceration, necrosis and sloughing may be seen, with ready haemorrhage and characteristic foetor'.

HIV-periodontitis was renamed necrotising (ulcerative) periodontitis (NUP) (ECClearinghouse, 1993) and defined as 'Periodontitis characterised by soft tissue loss as a result of ulceration or necrosis. Exposure, destruction or sequestration of bone may be seen, and the teeth may become loosened. Pain may be a prominent feature'. The conference noted that destruction could be extensive and that the disease was chronic and could be seen with ulceration during an active phase or without ulceration during a less active phase.

Little is known of these diseases. Only a small number of cases have been described and there is no information on their aetiology. Microbiological research may have been hampered by misdiagnosis (Chapter 4). Thus, the aims of this study were to characterise gingival ulceration seen in HIV-infection and to identify risk factors for its incidence, severity, extent, and recurrence. 


\subsection{Methods}

\subsubsection{Description of the cases}

\subsubsection{Selection of populations}

The setting for the study was a dental clinic dedicated to people with HIV infection. Patients described in this study were seen by any of the four dentists working on the clinic during the study period. Experience at the clinic suggested that gingival ulceration was not a frequent presentation. To provide sufficient information to describe the gingival ulceration comprehensively, a clinic protocol was introduced whereby all cases of ulceration involving the gingival margin in patients with HIV were recorded. The sample is therefore a consecutive sample of all cases of gingival ulceration seen in a five year period.

\subsubsection{Variables}

The variables considered in this study were:

i, Nature, extent and severity of lesions

ii, Teeth present

iii, Age

iv, Sex and route of transmission

v, Tobacco use

vi, Presence of oral manifestations of HIV infection

vii, Health status

viii, Neutrophil counts

ix, Co-trimoxazole use

$\mathrm{x}$, Management

xi, Microbiology

\subsection{Selection and development of measures}

To reduce measurement error of data recorded from clinical records variables were limited to those collected routinely or in clinic protocols and precision of measures. 


\subsection{Nature, extent and severity of lesions}

Some clinical description of the cases was required. As well as the descriptions placed in patient records as part of record keeping clinicians were asked to record the site of lesions, the severity of lesions (whether limited to the interdental papilla, beyond the interdental papilla or $10 \mathrm{~mm}$ or more from the CEJ) and whether bone was palpable, visible or sequestrating. To aid characterisation, lesions were photographed wherever possible.

\subsection{Teeth present}

The teeth present in each person with gingival ulceration were recorded to allow analysis of adjusted data for predilictions for particular sites.

\subsection{Age}

ANUG is a disease of young adults in the developed world. Age of patient was recorded in years from the patient records.

\subsection{Sex and route of transmission}

No data is available on the susceptibility to gingival ulceration by sex or transmission route. Route of transmission is recorded routinely based on patient self report as 'homosexual', 'heterosexual', 'injected drug use' or 'other'.

\subsection{Tobacco use}

ANUG is associated with tobacco use. Tobacco use is recorded routinely as part of clinical history of patients at the clinic. Where this data was absent in dental records the data were collected from medical records. Tobacco use was measured as 'ever' or 'never'.

\subsection{Presence of oral manifestations of HIV infection}

Clinic protocols demand that the presence of oral manifestations of HIV are recorded routinely as part of a standardised clinical examination. During the latter part of the data collection the diagnostic critieria used were those of the US Oral AIDS Collaborative group (Greenspan et al, 1992). Before October 1990 the clinic used criteria derived from Greenspan et al, (1986). Diagnostic criteria for the common oral manifestations were: 
Erythematous candidiasis; erythema of the palate, dorsum of the tongue or buccal mucosa not attributable to any other cause.

Pseudomembranous candidiasis; white patches anywhere in the mouth which could be removed by wiping.

Oral hairy leukoplakia; white patches on the lateral borders or ventral surfaces of the tongue which could not be wiped off and were not attributable to other causes.

Kaposi's sarcoma; purple or blue macules or papules anywhere in the mouth. Histopathology was required if the patient had not had a previous oral or extra-oral $\mathrm{KS}$ lesion confirmed by biopsy.

Ulceration; any oral ulceration not involving the gingival margin

Xerostomia; dry mouth manifest by fine leashes of bubbles and/or lack of salivary pooling.

\subsection{Health status}

History of opportunistic infections and tumours of HIV disease is recorded routinely in the notes as part of the medical history. Health status was recorded as a dichotomous variable by whether the patient had had a clinical indicator condition for AIDS.

Since 1990 CD4 counts have been used at most genito-urinary medicine clinics as a serological measure of HIV disease progression. Laboratories in London which analyse samples for this test have been calibrated to facilitate collaborative research. Lymphocyte differentials are repeated routinely every 3 months and the counts preceding presentation were recorded from patient's medical records. The primary clinical purpose of CD4 counts is to instigate primary prophylaxis against Pneumocystis carinii at a count of 200 cells $/ \mathrm{mm}^{3}$. Below this counts are repeated infrequently unless the patient is participating in a drug trial. If no count was available within 3 months, no record was made unless counts were below 50 cells $/ \mathrm{mm}^{3}$ in which case the last count was used regardless of date. Before 1990 CD4 lymphocytes were counted only if the patient was participating in a drug trial.

CD4 counts were used as a precise measure of HIV disease progression. To allow a larger data set to be analysed, a dichotomous measure; whether the patient met the 1993 case definition for AIDS was also used (Centers for Disease Control and Prevention, 1992). 


\subsection{Neutrophil counts}

Neutrophil counts are measured routinely every three months as part of the health screening of patients with HIV infection. The count preceding presentation was recorded. Counts more than three months before presentation were not used.

\subsection{Co-trimoxazole use}

Co-trimoxazole is the drug of choice for prevention and treatment of Pneumocystis carinii pneumonia and was suggested to be protective against NUP in one small study (Glick et al, 1994). Co-trimoxazole use was determined from medical history taken at the dental clinic. If no medical history was evident in the dental records within 3 months of the episode cotrimoxazole use was determined from patient's medical records.

\subsection{Management}

Clinic protocols specified the investigation and management of gingival ulceration. At presentation all gingival ulcers were debrided to remove slough, gross calculus, plaque and any sequestra and then irrigated with $10 \mathrm{ml}$. of $0.2 \%$ aqueous chlorhexidine solution via a blunt syringe. Patients were prescribed three days of metronidazole $200 \mathrm{mg}$. tds. and chlorhexidine mouthrinses bd. then reviewed one week later. This was followed by intensive oral hygiene instruction and scaling and polishing. Patients were then recalled to the hygienist every 3 months. Resolution was defined as relief of symptoms and lack of ulceration.

\subsection{Microbiology}

Murray and Winkler (1988) had found the levels of spirochaetes present in what they called HIV-P to be variable and not consistent with the microflora associated with ANUG. To investigate this phenomenon curettings from the initial debridement were placed on to glass slides, heat sealed and Gram stained using 1:10 carbol fuchsin as the counter-stain. The number of spirochaetes and fusiform bacteria present was scored on an ordinal scale of 'none', 'few', 'moderate' and 'numerous'.

\subsubsection{Data Analysis}

Univariate analyses were used to identify factors associated with number of sites affected, severity of episode and relapse. Episodes with ulceration which extended $10 \mathrm{~mm}$ or more 
from the CEJ or visibly or palpably exposed bone were classified as severe. Relapse was defined as presentation at the clinic with a second episode. Cases involving five or more ulcerated sites were dichotomised as 'many' and those with 4 or less were considered 'few'.

\subsubsection{Cases and controls}

To provide data on the distribution of exposures among people with HIV without ulceration a control group was selected from unaffected patients attending the clinic. The controls were therefore subject to the same selection factors as the cases.

Preliminary analysis of the data suggested there were at least 80 cases with a single diagnosis. Using this approximation, a case:control ratio of $1: 3$ would be required to lend $80 \%$ power to detect an odds ratio (OR) of 2.2 at an alpha of 0.05 in regression models (Schlesselman, 1982). Therefore the records of the 6 consecutive patients with HIV examined after each case were reviewed and the three with the most complete data were selected as controls. This strategy aimed to sample a control group which represented the clinic population during the study period without the introduction of a cohort effect and minimised the risk of recall and other forms of measurement error. Controls were not matched to cases for any other factor than date of presentation since matching may introduce confounding into case control studies. If the controls are matched to the cases for a factor correlated to the exposure then the exposed proportion in the controls is distorted (Rothman, 1986). Using this method the final sample size for the case control study was 358 , comprising 89 cases and 269 controls.

To prevent bias, variables were limited to information collected routinely and regularly from patients and included age, sex, HIV transmission route, health status, tobacco use, cotrimoxazole use and the health of the oral mucosa.

\subsubsection{Data analysis}

Data for CD4 counts were skewed and were transformed to root CD4 count to allow parametric and regression analyses. Appropriate univariate analyses were used to test for possible associations between independent variables and the presence of ulceration. Associations for which the null hypothesis had a probability of less than 0.2 were tried in forward stepwise logistic regression models (JMP 2.0, SAS Institute Inc., Cary, NC, USA). A probability of $5 \%$ was used as the level of significance in regression models. 
The numbers of women, heterosexual men and injected drug use controls were very small and co-trimoxazole is used only in late HIV disease. Therefore regression analysis of the effect of these variables was precluded. Their effects were tested by stratification. Since the numbers involved were so small this effectively meant excluding women, heterosexual men and drug users from the regression analyses and observing the effect on the models.

\subsection{Results}

In the 5 years between August 1988 and August 1993, 94 (7.1\%) of the 1308 patients with HIV seen at the clinic had one or more episodes of gingival ulceration.

\subsubsection{Description of the cases}

The cases fell into two distinct groups: 89 (95.7\%) patients had ANUG-like lesions which responded readily to the treatment regimen and $5(5.4 \%)$ had ulceration which did not respond to treatment but subsequently responded to treatment for neutropenia.

Eighty-nine patients (6.9\% of the total seen) had 140 episodes of ulceration which responded to treatment. These are the cases in the case control study. All 89 were homosexual or bisexual men. Mean age at presentation with the disease was 32.4 years (SD 6.48 years). Twenty-four met the case definition for AIDS (Centers for Disease Control and Prevention, 1992). Data for CD4 counts were skewed. Median CD4 count was 320 cells $/ \mathrm{mm}^{3}$ with a $95 \%$ central range of $13-1118$ cells $/ \mathrm{mm}^{3}$. The condition of the oral mucosa was recorded in 67 cases of whom $56.7 \%$ had oral candidiasis, $44.8 \%$ had oral hairy leukoplakia, $1.5 \%$ had Kaposi's sarcoma and $4.5 \%$ had ulceration of the oral mucosa.

Forty-eight (53.9\%) were current smokers, 24 (27.0\%) were not. Data on this variable was not available for the remaining $17(19.1 \%)$ men.

Recording of symptoms was not part of the protocol and was inconsistent in the records. However, several cases were noted to be "pain free" and there were no recordings of the "deep" or "bone" pain described by Winkler et al. (1987). Patients usually complained of "sore gums" and sometimes "as if something was wedged between the teeth".

Patients presented with ulceration and necrosis of the gingivae which invariably appeared to be radiating from the inter-dental papillae with a soft grey slough or pseudomembrane. 
Figure 6.1. Gingival ulceration in a 47 year old bisexual man with HIV infection.

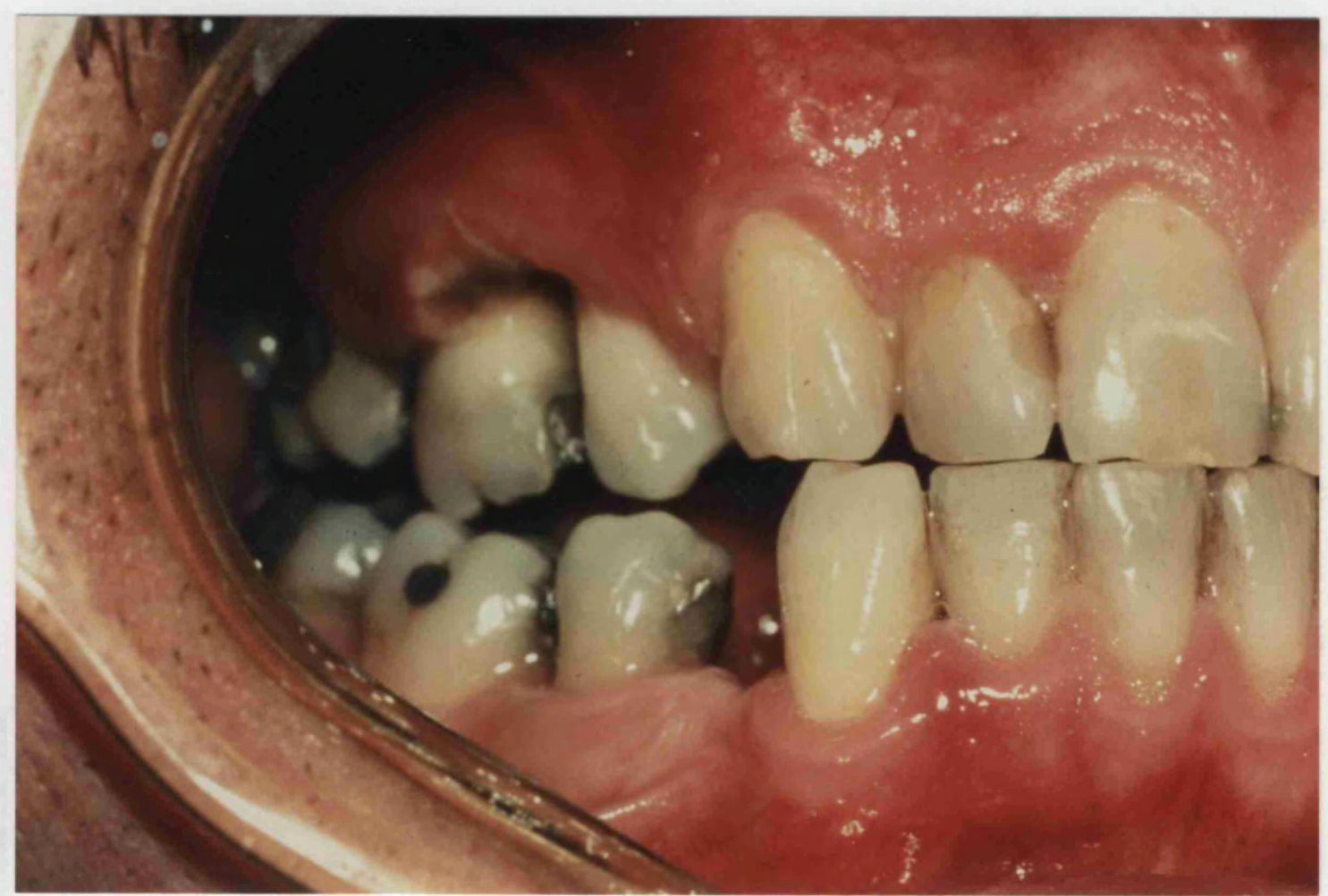


Lesions bled spontaneously or readily on instrumentation and there was always a characteristic foetor of ANUG. Figure 6.1 depicts such a case in a 47 year old bisexual man with ulceration between the upper first molar and premolar. There is also mild ulceration of the papilla tip mesial to the lower canine.

Figure 6.2 presents the frequency distribution for the number of sites affected in the first episode. Up to thirty-four 'teeth' were affected (mean 6.2, median 4, 95\% central range 128). Figure 6.3 shows the frequency distributions for the sites of ulceration. Both the lower first and second incisors were affected in almost half the episodes and the upper central incisors were affected in one third of the episodes.

The severity of ulceration was recorded in 79 (88.8\%) cases. Ulceration was limited to the papillae in $26(29.2 \%)$ cases, in some cases only the papilla tips were affected. In 43 (48.3\%) cases ulceration extended beyond the papilla to create marked interdental cratering. Necrosis extended $10 \mathrm{~mm}$. or more from the cemento-enamel junction in $4(4.5 \%)$ cases and bone was either visible or palpable in another $6(6.7 \%)$. These 10 cases were ranked as 'severe' and all other cases were considered 'mild'.

The frequency distribution for the number of episodes experienced by the group is shown in Table 6.1. Thirty (33.7\%) of the eighty-nine attended the clinic with a relapse during the study period.

Table 6.1. Frequency distribution for number of episodes among 89 patients with gingival ulceration.

\begin{tabular}{|c|c|}
\hline No. of episodes & Frequency \\
\hline 1 & 59 \\
2 & 20 \\
3 & 5 \\
4 & 2 \\
5 & 0 \\
6 & 3 \\
\hline
\end{tabular}


Figure 6.2. Frequency distribution for the number of sites affected in first episode of gingival ulceration.

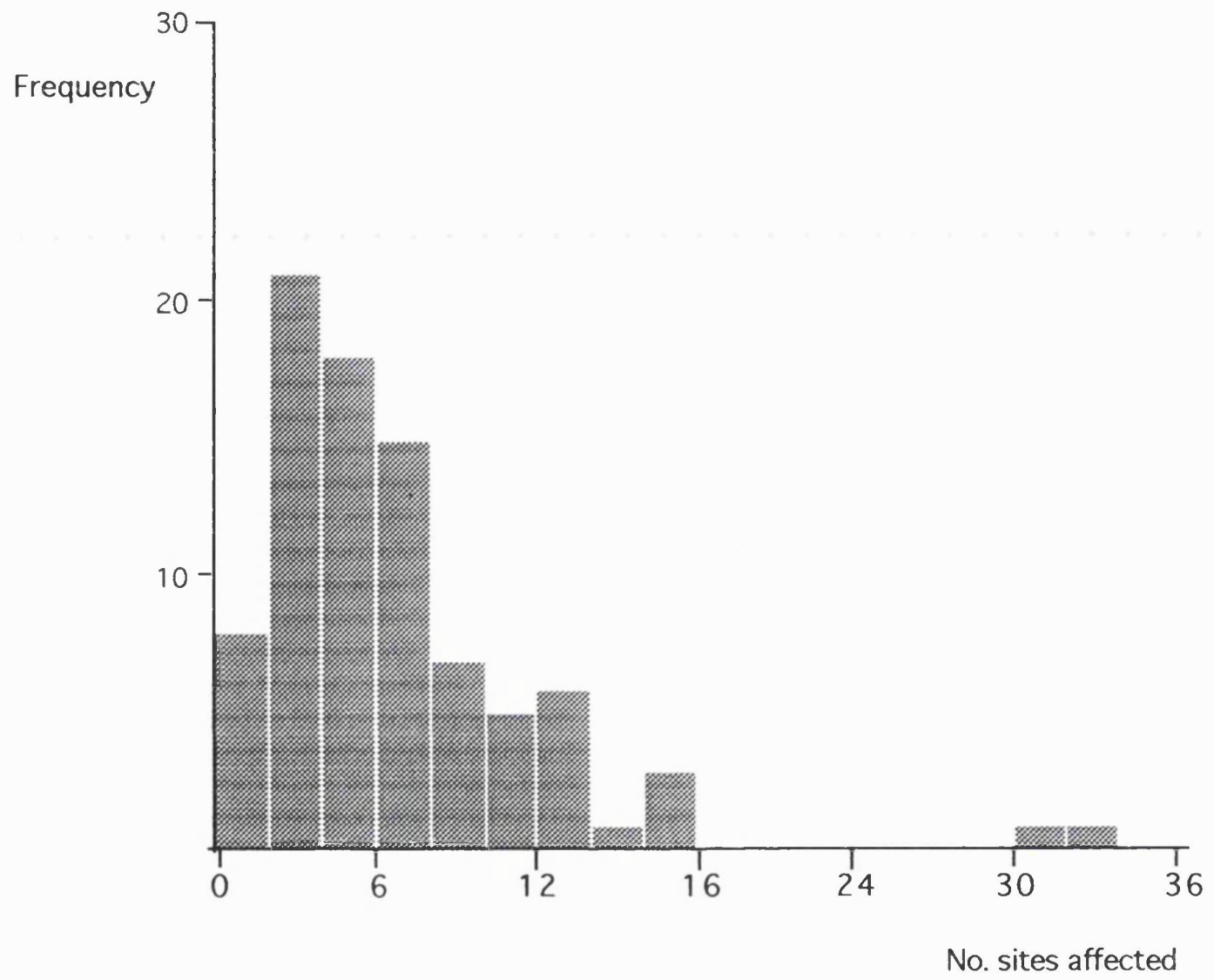


Figure 6.3. Frequency distribution of teeth affected in first episode of gingival ulceration, corrected for teeth present.

Frequency

(Proportion

of cases)

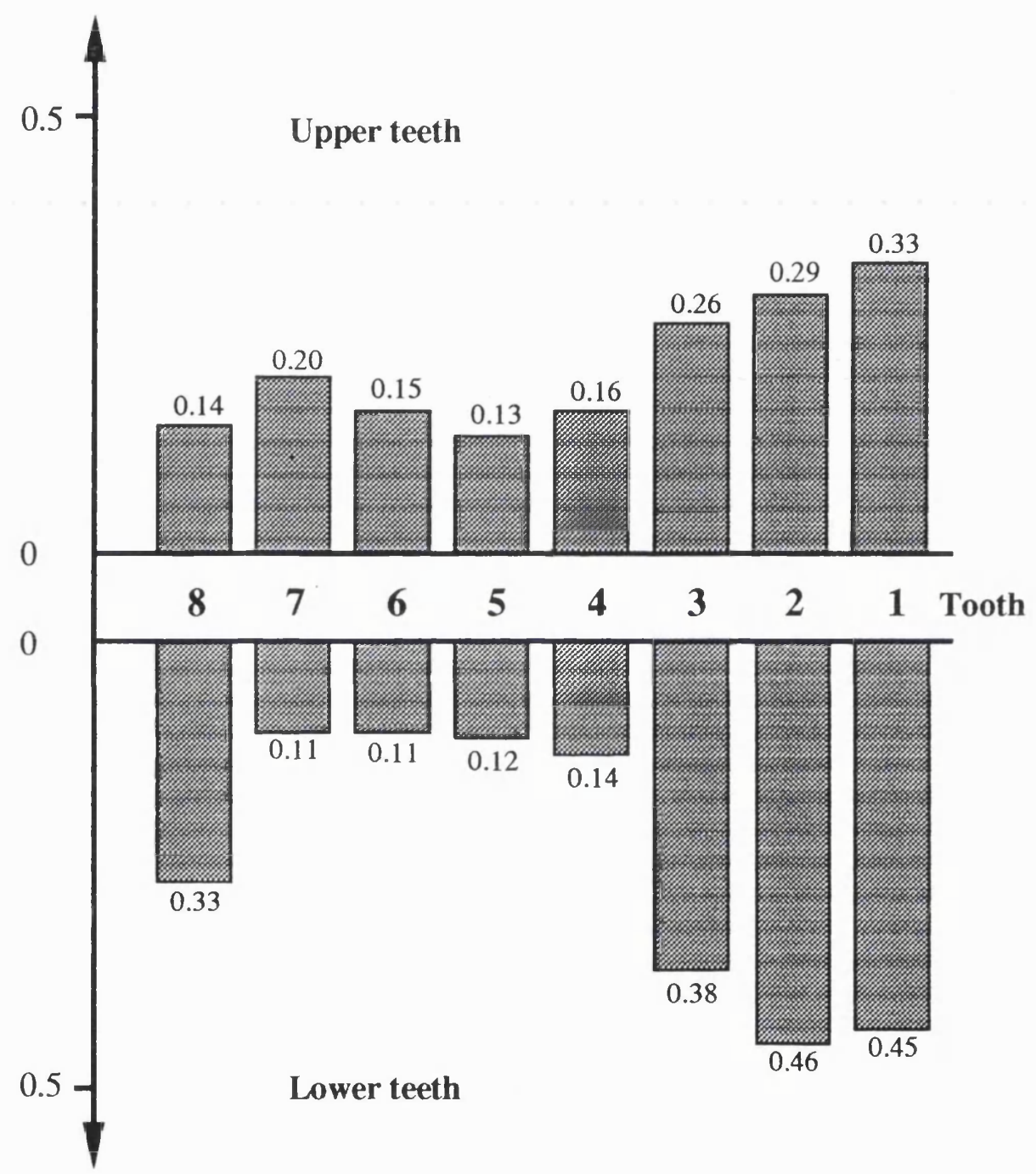


Tables 6.2 to 6.4 summarise the independent variables in mild/severe, single episodes/relapsed cases and extensive/non-extensive cases. Linear regressions for the extent of ulceration by age and neutrophil count are presented in Appendices 16 and 17. Half of the 10 severe cases were men with AIDS compared to 19 of the 79 mild cases $(P=0.081$, Chi Sq.). No other positive associations were found in the number of sites, severity of case or relapse with age, severity of immunosuppression (dichotomised about the case definition for AIDS), neutrophil count, tobacco use and presence of other mucosal disease.

Some patients reported symptomatic relief immediately after curettage. Eighty-four cases returned for 1 week review, of whom 79 had resolved completely (94\%). The remaining 5 cases had had ulceration extending beyond interdental papillae, 4 of which had resolved within 3 weeks. Resolution of the final case was hindered by the presence of a large piece of sequestrum wedged between the teeth which could not be removed until 4 weeks after presentation. This patient remained asymptomatic after initial curettage. Figures 6.4 and 6.5 show the response to treatment in the man depicted in Figure 6.1. One week after initial debridement the inflammation and sloughing is reduced but a small mobile sequestrum is visible. This was removed. Two years later a significant defect persisted but caused no further problems.

Curettings from debridement of the ulcers of 47 patients were analysed and moderate or numerous numbers of fusiforms and spirochaetes were present on all 47 .

Table 6.2. Independent variables in mild and severe episodes of gingival ulceration

\begin{tabular}{|l|cc|c|}
\hline & $\begin{array}{c}\text { Mild } \\
(\mathrm{n}=79)\end{array}$ & $\begin{array}{c}\text { Severe } \\
(\mathrm{n}=10)\end{array}$ & $P$ \\
\hline Mean age (y) (SD) & $32.2(6.5)$ & $34.1(6.5)$ & $0.376, \mathrm{t}$ test \\
Mean neutrophil & $3.1(1.5)$ & $2.3(1.5)$ & $0.158, \mathrm{t}$ test \\
count x 109/L(SD) & & & \\
Prop. with AIDS (\%) & 24.1 & 50.0 & 0.082, Chi sq. \\
Current tobacco users & 65.6 & 75.0 & 0.596, Chi sq. \\
(\%) & & & \\
Oral candidiasis (\%) & 55.9 & 62.5 & 0.725, Chi sq. \\
Hairy leukoplakia & 44.1 & 44.8 & 0.751, Chi sq. \\
(\%) & & & \\
\hline
\end{tabular}


Table 6.3. Independent variables in cases of gingival ulceration who did and did not relapse

\begin{tabular}{|l|cc|c|}
\hline & $\begin{array}{c}\text { Relapse } \\
(\mathrm{n}=29)\end{array}$ & $\begin{array}{c}\text { No Relapse } \\
(\mathrm{n}=60)\end{array}$ & $P$ \\
\hline Mean age (y) (SD) & $31.4(6.5)$ & $32.9(6.5)$ & $0.317, \mathrm{t}$ test \\
Mean neutrophil count x & $3.1(1.6)$ & $2.3(1.6)$ & $0.158, \mathrm{t}$ test \\
109/L(SD) & & & \\
Prop. with AIDS (\%) & 24.1 & 28.3 & 0.676, Chi sq. \\
Current tobacco users (\%) & 61.5 & 69.6 & 0.488 , Chi sq. \\
Oral candidiasis (\%) & 52.2 & 59.1 & 0.587, Chi sq. \\
Hairy leukoplakia (\%) & 44.8 & 47.7 & 0.502, Chi sq. \\
\hline
\end{tabular}

Prop. $=$ proportion

$P=$ probability

Table 6.4. Median numbers of sites ulcerated by severity of HIV disease, oral diseases and current tobacco use

\begin{tabular}{|l|cc|c|}
\hline & Present (95\% CR) & Absent (95\% CR) & $P$ (MWWT) \\
\hline AIDS diagnosis & $6(1-32)$ & $4(1-22)$ & 0.437 \\
Oral candidiasis & $4(1-17)$ & $6(1-32)$ & 0.541 \\
Hairy leukoplakia & $6(1-17)$ & $4(1-32)$ & 0.857 \\
Current tobacco use & $5(1-32)$ & $5(1-32)$ & 0.718 \\
\hline
\end{tabular}

$\mathrm{CR}=$ central range

$P=$ probability

MWWT = Mann Whitney Wilcoxon Test. 
Figure 6.4. Lesion in Figure 6.1 one week after debridement. Sequestrum is visible.

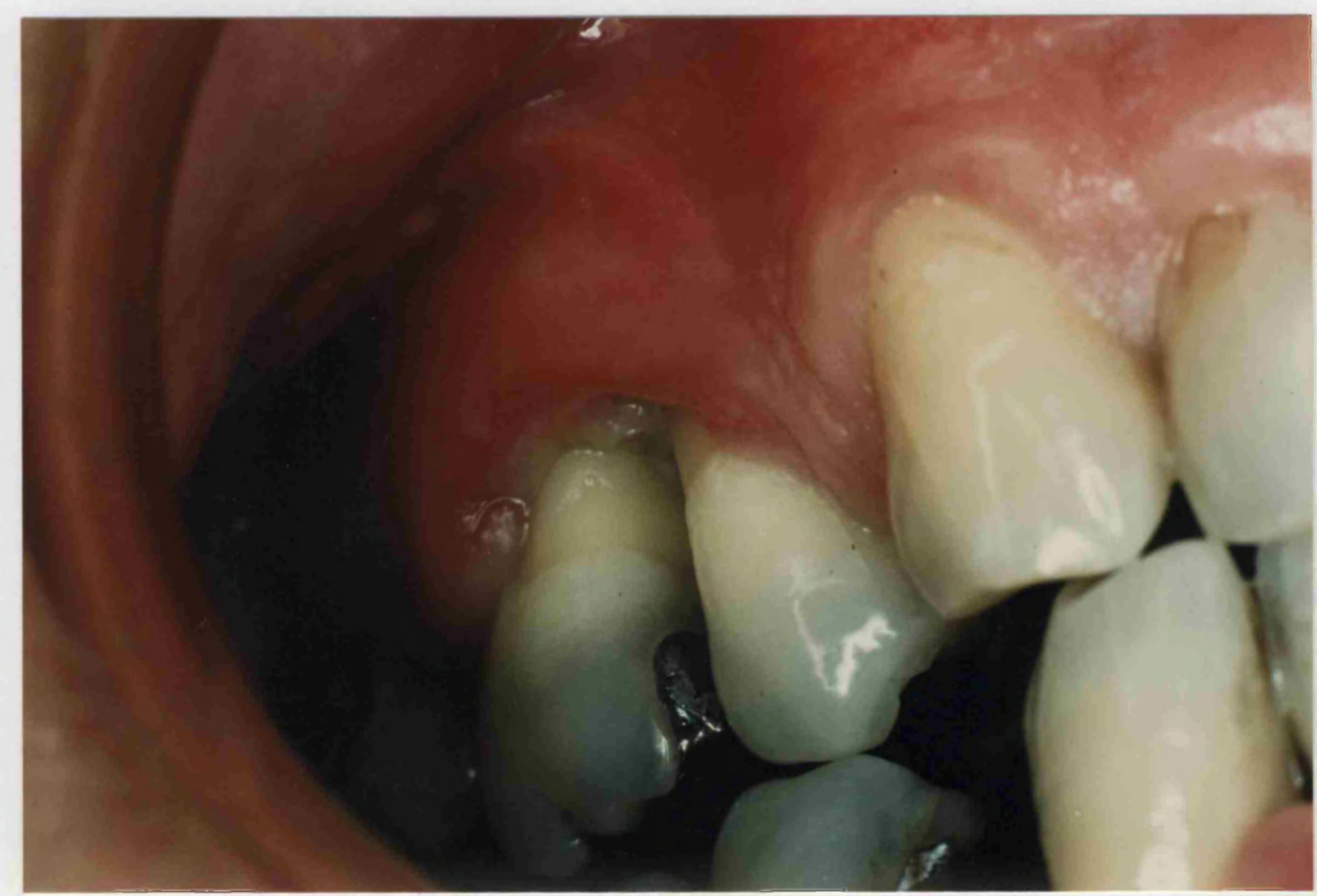


Figure 6.5. Same site as Figures 6.1 and 6.4 two years later.

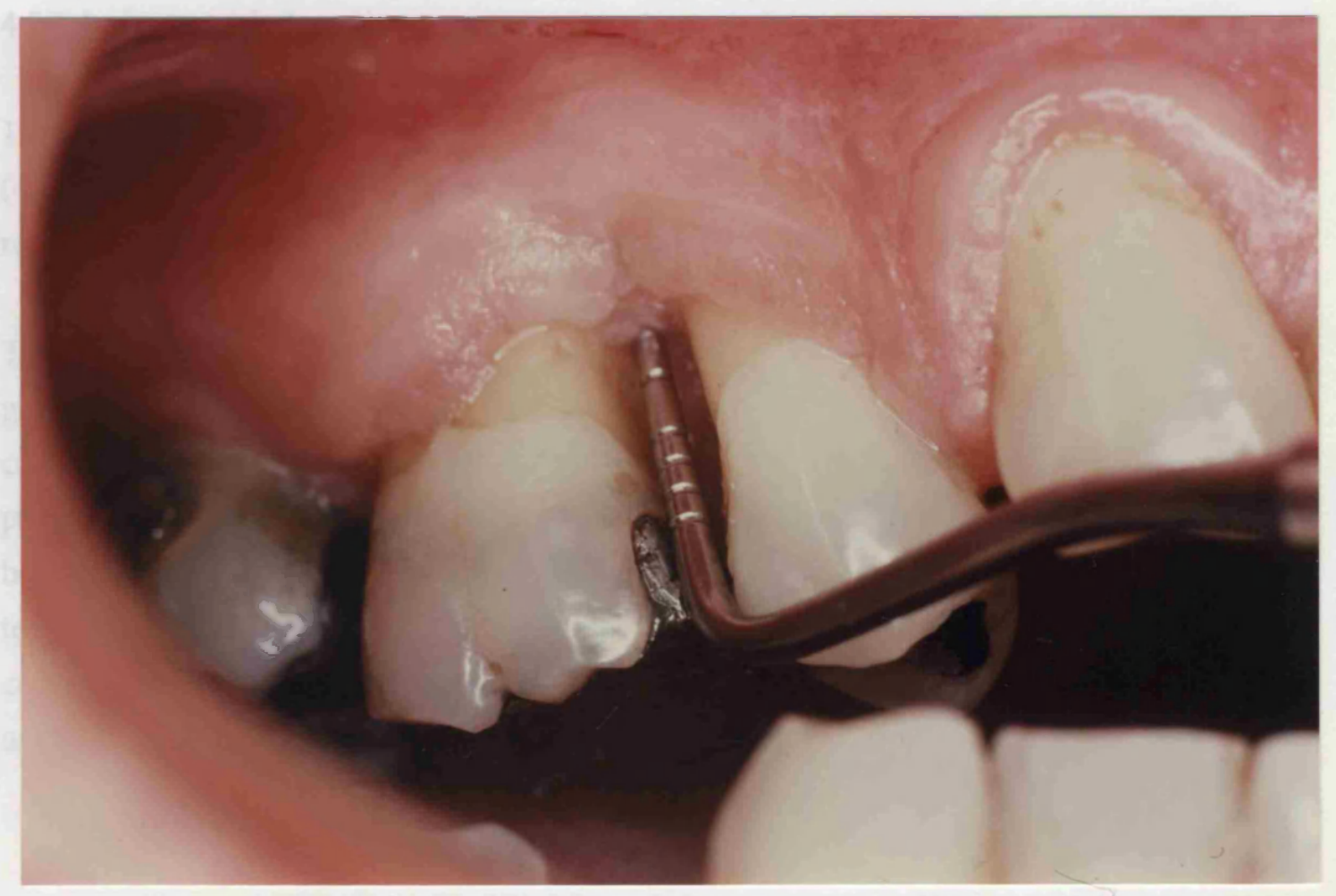

The probe has Williams markings and the tip can be moved between the two teeth $7 \mathrm{~mm}$ from the cemento-enamel junction. 


\subsubsection{Case control study}

Two hundred and sixty-nine controls were selected to compare with the 89 cases (mean age 36.1 years, SD 8.2 years). Eleven were women. Four of the 258 men had been drug users and one had acquired HIV from a female sexual partner. The remaining 253 were homosexual men.

117 controls met the case definition for AIDS (43.5\%). Median CD4 count was 270 cells $/ \mathrm{mm}^{3}$ with a $95 \%$ central range of $10-835$ cells $/ \mathrm{mm}^{3}$. Mean neutrophil count was 2.5 x $10^{9}$ cells/L (SD 1.29). Mucosal health data were available for 267 controls of whom $27.3 \%$ had oral candidiasis, 33.3\% had hairy leukoplakia, 5.9\% had Kaposi's sarcoma and 4.5\% had mucosal ulceration.

133 controls were current smokers, 82 had never smoked and 20 had smoked in the past (49.4\%, 30.5\% and $7.4 \%$ respectively). Data were not available on tobacco use for the remaining 34 controls (12.6\%).

Table 6.5 presents the best forward stepwise logistic regression model for predictors of gingival ulceration among the cases and controls (total 358). The presence of oral candidiasis (odds ratio 3.4), being younger and not having AIDS (OR 2.1) strongly predicted the presence of gingival ulceration. Six other independent variables were tested but excluded by the regression model including: neutrophil count; sex; presence of OHL; tobacco use; presence of KS and CD4 count (see Appendix 18). Excluding members of the control group who were not homosexual men did not significantly alter the results of the analysis (See Appendix 18).

Table 6.5. Best forward stepwise logistic regression model for independent variables related to the presence of gingival ulceration among 89 cases and 269 controls.

\begin{tabular}{|lllc|}
\hline$R^{2}=0.103$ & \multicolumn{2}{c|}{} \\
Intercept & $b$ & se & \multicolumn{1}{c|}{} \\
Age & 0.588 & 0.722 & 0.415 \\
Candidiasis & -0.057 & 0.021 & 0.007 \\
Not AIDS & 0.667 & 0.147 & $<0.001$ \\
\hline
\end{tabular}

$b=$ regression coefficient

se $=$ standard error

$R^{2}=$ proportion of variation explained by the model 
At the time of presentation 24 cases were eligible for co-trimoxazole use (based on meeting the case definition for AIDS). Data were available for 21 of these of whom 3 were using the drug. Data were available for 117 eligible controls of whom 51 were using cotrimoxazole $(P<0.05$, Chi sq. and $95 \% \mathrm{CI}=0.122$ to 0.465$)$.

If patients using co-trimoxazole were excluded from the analysis 85 cases and 218 controls remained. The relationship between lack of AIDS diagnosis and presence of ulceration was no longer apparent in univariate analysis or the regression model (Table 6.6). This data is presented graphically in Figure 6.6. The incidence of gingival ulceration among those with early disease or with AIDS but not using co-trimoxazole were similar and greater than the incidence among patients with AIDS who were using co-trimoxazole $(30 \%, 23 \%$ and $6 \%$ respectively).

\subsubsection{Cases which did not respond to treatment.}

Five patients had 6 episodes which did not respond to the treatment regimen. Four were homosexual men and the other was a woman who had acquired HIV from a male sexual partner. One of the homosexual men had asymptomatic HIV disease apart from the oral lesions, a CD4 count of $380 \times 10^{9}$ cells $/ \mathrm{L}$ and neutrophil count of $0.9 \times 10^{9}$ cells/L. The remaining 4 patients had AIDS, CD4 counts of less than 50 and neutrophil counts of 0.3 , 0.6 ( 2 men $)$ and $1.0 \times 10^{9}$ cells/L.

All 5 patients had large shallow leathery ulcers at the gingival margins which did not bleed readily on curettage. No spirochaetes or fusiforms were detected on smears. Viral and bacterial culture, and histopathology did not aid diagnosis. All five patients responded to treatment of their neutropenia. 
Table 6.6. Best forward stepwise regression model for gingival ulceration among 279 patients not taking co-trimoxazole

\begin{tabular}{|llll|}
\hline$R^{2}=0.092$ & & & \\
Intercept & $\boldsymbol{b}$ & se & \multicolumn{1}{c|}{$\boldsymbol{P}$} \\
Age & 0.729 & 0.746 & 0.328 \\
Candidiasis & -0.053 & 0.022 & 0.013 \\
Not AIDS & 0.663 & 0.152 & $<0.001$ \\
\hline
\end{tabular}

$b=$ regression coefficient

$\mathrm{se}=$ standard error

$R^{2}=$ proportion of variation explained by the model

Figure 6.6 Relative frequency of gingival ulceration among patients with AIDS using and not using co-trimoxazole and patients with early HIV disease.

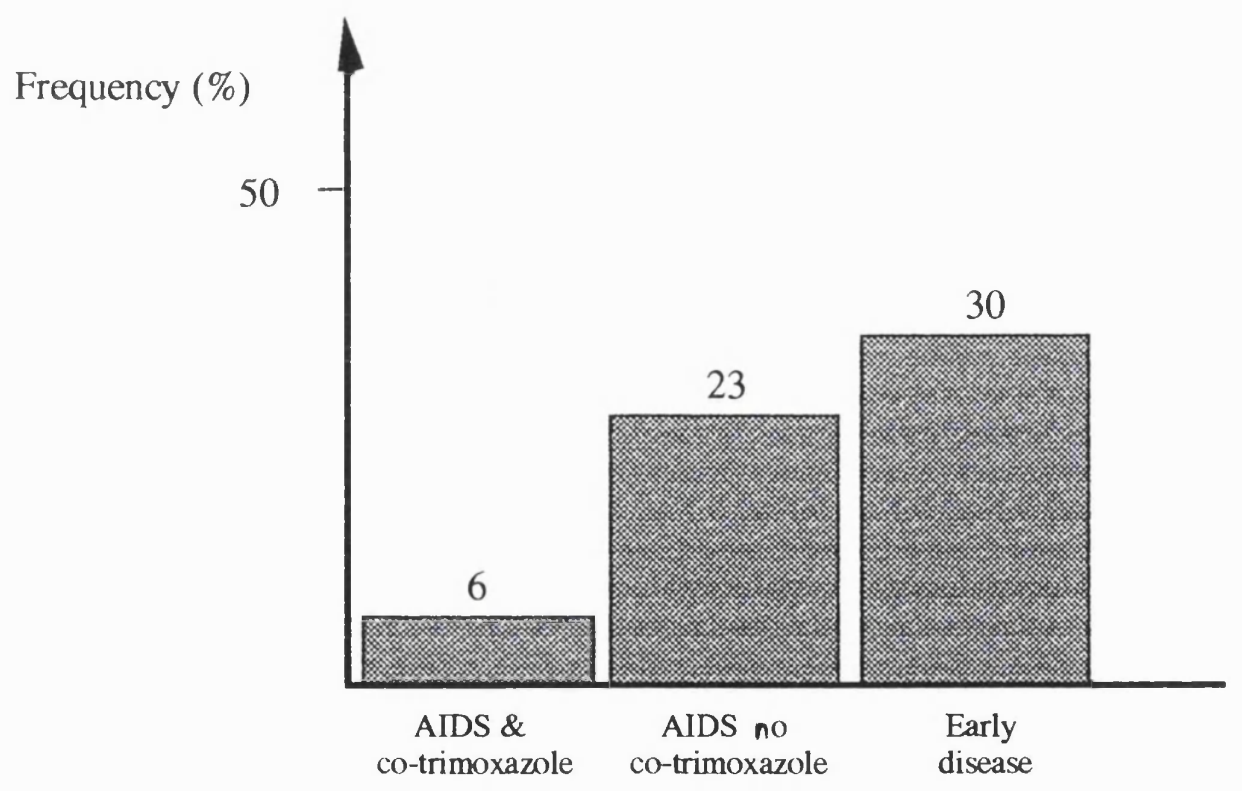




\section{$6.5 \quad$ Discussion}

Two different types of gingival ulceration were seen in a group of dental patients with HIV: a disease similar to ANUG and ulceration with no identifiable cause. The ANUG-like disease was seen in $95 \%$ of cases with ulceration. It presented as ulceration with a soft slough or pseudomembrane radiating from the inter-dental papillae. All cases had the characteristic foetor oris associated with ANUG. In all cases sampled moderate or numerous fusiforms and spirochaetes were visible on Gram staining and the lesions responded readily to an anti-microbial regimen. Although the symptoms differ from those described by Winkler et al. (1988) this is presumably the disease previously described as AIDS virus associated periodontitis, HIV-periodontitis and necrotising ulcerative gingivitis/necrotising ulcerative periodontitis. This description agrees very closely with descriptions of ANUG reviewed by Johnson et al (1986).

The findings of the present study suggest that .NUG, NUP and ANUG are the same disease. Winkler \& Murray (1987) distinguished NUP (formerly HIV-P) from ANUG by its rapid progression and because ANUG is self-limiting. This may not be sufficient justification for distinguishing the two different presentations since ANUG may simply progress more rapidly in HIV-infected people. The disease is not self-limiting in some African children (Emslie, 1963; Sheiham, 1966; Osuji, 1990). Murray et al. (1988) found the levels of spirochaetes present in NUP to be variable and not consistent with the microflora associated with ANUG but their findings may have resulted from misdiagnosis (see Chapter 4).

Neither do the available data on symptoms of disease differentiate between the condition observed and ANUG. In particular there were no records of the "deep" or "bone" pain described by Winkler et al. (1987). Limited inference can be drawn from this as recording of symptoms was not standardised. However, "bone pain" is a very infrequent symptom among patients at other clinics providing care for people with HIV infection in London and New York (Guy Palmer and Charles Barr, personal communications). It may be that too much emphasis has been placed on a small number of anecdotal reports in the past.

In a five year study period, less than $7 \%$ of the patients attending a dedicated dental clinic had NUG or NUP. Ulceration was limited to the papillae in $29 \%$ of cases (NUG) and extended beyond the papilla in $48 \%$ of cases (NUP)(EC-Clearinghouse, 1993). These percentages do not reflect the incidence because the sample was self-selected for symptomatic dental disease and because the clinic population was dynamic. However, the data suggest the annual incidence of NUG/NUP in people with HIV is less than $1.5 \%$. This 
is very low compared to prevalence studies of similar periodontal lesions in infected populations (Zakarian et al, 1988; Klein et al, 1991; Murray et al, 1991; Swango et al, 1991; Laskaris et al, 1992; Masouredis et al, 1992; Thompson et al, 1992) but close to the $6.3 \%$ experience of NUP over 4 years among 700 attenders at a similar dedicated clinic (Glick et al, 1994).

The differences between the findings of this and other studies may be accounted for by differences in diagnostic criteria (Swango et al, 1991; Masouredis et al, 1992), route of HIV transmission (Klein et al, 1991; Murray et al, 1991) and patient selection (Laskaris et $a l, 1992)$. In addition, some of the patients in this study were regular attenders at the clinic and may have benefited from regular preventive treatment. One other contributory factor for the apparent disagreement between the incidence data and prevalence studies is the acute nature of treated NUG/NUP. The prevalence of an acute recurrent disease is more closely related to the number of episodes than the number of individuals affected. The 89 cases had 140 episodes of NUG/NUP and the disease would appear proportionately more frequent in prevalence studies.

Whilst less than $7 \%$ of patients had NUG/NUP, one third of these had 2 or more episodes during the study period and 3 had 6 episodes (Table 6.1). This appears to be a population at high-risk for the disease.

Less than 5 teeth were affected in most cases and the lower incisors were affected in almost half the cases (Figures 6.1 and 6.2). Smith et al. (1995) found a similar distribution of attachment loss among a small group of patients with HIV. No predilection for sites has been documented for gingival ulceration in people with HIV or for ANUG in general populations.

There was a tendency for more patients with more advanced HIV disease to have more severe NUP $(P=0.08)$ but there were no other discernible relationships between the severity, extent or relapse of NUG/NUP with age, severity of immunosuppression, neutrophil counts, tobacco use or the presence of other mucosal disease. Such relationships may exist but the sample size of 89 people may be too small to detect them especially in the presence of intervening variables such as access to care. However, the lack of association between any of these variables and the severity of ulceration supports the concept that NUG and NUP are the same disease and it seems logical that unremitting gingival ulceration will become periodontal ulceration. 
The treatment regimen provided immediate symptomatic relief in some cases and resolution within 1 week in $94 \%$ of cases. Ulceration persisted in severe cases especially when complicated by sequestrum, although there was relief of symptoms. The treatment regimen is similar to that described by Winkler at al. (1989). In the present study, lesions were perfused with aqueous chlorhexidine with no apparent ill effects and metronidazole was prescribed at a lower dose, less frequently and for a shorter period. These are the most comprehensive and systematic data reported so far on the acute management of ANUG in HIV infection. The treatment was effective, efficient and easily tolerated in the acute management and provides a benchmark against which other treatments can be compared.

In the case control study, 89 cases of ANUG were compared with 269 other clinic patients with HIV. The incidence of ANUG was associated with the presence of oral candidiasis, lower age and not having an AIDS diagnosis.

Although significantly younger than the controls, patients with ANUG were older than might be expected. This probably reflects the age of the patients attending the clinic, the vast majority of whom had a sexually transmitted disease they would not be expected to acquire when very young. In industrialised countries ANUG is regarded as a disease of young adults although there is little recent evidence to support this. Most epidemiological studies in the last 50 years were of populations of university students or army recruits (Pindborg, 1951; Giddon et al, 1964). In a review of 61 cases of recurrent ANUG, Manson found a third were more than 25 years of age (Manson and Rand, 1961).

Oral candidiasis was a strong predictor of the incidence of ANUG and its effect was independent of severity of immunodeficiency as measured by CD4 counts or AIDS diagnosis. Oral candidiasis is also associated with erythema of the attached gingiva and possibly with linear gingival erythema in people with HIV (Grbic et al, 1995; Chapter 5). Studies of the subgingival microflora seen in HIV infection have detected Candida spp. at higher carriage rates than seen in adults with severe periodontitis (Murray et al, 1988; Slots et al, 1988; Zambon et al, 1990; Moore et al, 1993).

Candidal hyphae and pseudohyphae have been detected in gingival biopsies from people with HIV but not in HIV negative control samples (Odden $e t a l, 1994)$. Candidal invasion was more frequent in people who had had necrotising periodontal disease. Odden et al. (1995) have detected fewer CD4 lymphocytes in the periodontal connective tissue of HIV positive subjects with a history of "NUP" compared to HIV positive controls without. Absolute gingival lymphocyte counts reflected the lymphocytopenia of HIV disease but CD4/CD8 ratios were increased in gingival samples from most subjects with HIV which 
suggested active recruitment of CD4 lymphocytes to the gingiva. Odden et al. (1995) suggested that the reduced numbers of local CD4 lymphocytes could explain the candidal and bacterial invasion. Site of oral candidiasis was not recorded in the present study but infection is often in the palate, buccal mucosa and dorsum of the tongue (ECClearinghouse, 1993). Taken together, this implies that the CD4 lymphocyte deficiency is localised to the mouth not just the periodontium. An alternative hypothesis is that Candida spp. are implicated in the pathogenesis of ANUG rather than merely confounders as Odden et al. (1994) suggested.

ANUG was associated with less severe HIV disease as measured by AIDS diagnosis. Other studies have produced conflicting results. Necrotising periodontal disease was more common in the absence of AIDS in one study and more extensive at higher CD4 counts in another (Swango et al, 1991; Palmer et al, 1996). Two smaller studies found necrotising disease to be more common with lower CD4 counts (Thompson et al, 1992; Glick et al, 1994). Methodological issues may be responsible for the differences. For example, Glick $e t$ al. (1994) reported a case control-study of patients with NUP and did not describe how their controls were selected.

The inverse relationship between ANUG and AIDS seems to be due to the use of cotrimoxazole which was protective against ANUG in univariate analysis and accounted for the inverse relationship with HIV disease progression in stratified analysis (Table 6.6). The drug is used in advanced HIV disease to prevent and treat Pnemocystis carinii pneumonia (PCP) (Centers for Disease Control and Prevention, 1992).

Glick et al. (1994) reported a similar finding when co-trimoxazole use was compared between 40 patients with "NUP" and AIDS and 35 similar patients without "NUP". This suggests that some of the organisms involved in ANUG are sensitive to co-trimoxazole. Anaerobic bacteria (particularly Bacteroides spp. and presumably the oral relations Porphyromonas and Prevotella) are sensitive to sulphonamide/trimethoprim combinations (Kucers and Bennet, 1987). The protective effect of co-trimoxazole supports anecdotal reports that the frequency and nature of localised destructive disease has changed since the early days if the HIV epidemic (Winkler, 1995). The effect of co-trimoxazole may also explain why AIDS diagnosis was more strongly related to ANUG than CD4 counts.

The lack of association between tobacco use and ANUG is surprising since the two are strongly associatedin other populations (Johnson and Engel, 1986). The literature again contains conflicting findings relating to people with HIV. Necrotic destruction was more 
extensive in smokers in the study by Swango et al. (1991) $(P=0.06$ and $P=0.03)$ but of similar frequency in the study by Glick et al. (1994). Tobacco use may be a predictor for ANUG but its effect may be masked by more potent predictors such as candidiasis.

Five patients had 6 episodes of ulceration of the gingiva for which a diagnosis could not be made and are assumed to have had 'Ulceration not otherwise specified' (Ulceration NOS). This is a diagnosis of exclusion used in HIV infection (EC-Clearinghouse, 1993). The ulceration is thought to have been mucosal ulceration but was considered in this study because all cases of ulceration involving the gingival margin were included. All five patients were neutropenic and their ulceration responded to treatment of the underlying neutropenia.

Approximately $8 \%$ of people with asymptomatic HIV infection are neutropenic (Zon and Groopman, 1988) either due to ineffective neutrophil production, neutrophil antibodies or medications (McCance-Katz et al, 1987; Israel and Plaisance, 1991). Neutrophil activity is also impaired through a combination of decreased random migration, chemotaxis, adhesion, bacterial killing and phagocytosis (Ras et al, 1984; McCance-Katz et al, 1987; Ellis et al, 1988; Elbim et al, 1994; Flø et al, 1994) which are present in early HIV infection but more pronounced in later disease (Roilides et al, 1993; Flø et al, 1994). The cumulative effect of neutrophil impairment, neutropenia and other immune system defects may increase susceptibility to bacterial and fungal infections (Elbim et al, 1994; Flø et al, 1994). Roilides et al. (1993) have suggested that this cumulation of defects may allow infections with milder neutropenia than seen in otherwise healthy patients.

Oral ulceration has been described in people with primary PMN defects (Porter and Scully, 1993) and this suggests that some of the non-specific ulceration seen in HIV infection may be related to neutrophil depletion or impairment.

\subsection{Conclusions}

This case series and case control study found gingival ulceration to be uncommon among dental patients with HIV.

The majority of cases are clinically and microbiologically similar to ANUG and respond to conventional treatment of that disease. The lower and anterior teeth are most frequently affected. One third of affected patients subsequently relapsed, despite maintenance with a hygienist. This suggests that this sub-group might be particularly susceptible to ANUG. 
The severity, extent and relapse of ulceration was not related to age, disease status, neutrophil counts, tobacco use or the presence of other mucosal disease. The incidence of ANUG in HIV infection is associated with the presence of oral candidiasis, lower age and lack of AIDS diagnosis. Co-trimoxazole appears to be protective against the disease. There is no relationship between AIDS and the incidence of ANUG among patients not taking this drug.

A minority of cases resemble the Ulceration NOS seen in patients with HIV infection but are associated with neutropenia in the absence of any apparent infectious cause. 
CHAPTER 7 THE VALIDITY OF DIAGNOSTIC CRITERIA FOR PERIODONTAL CHANGES ASSOCIATED WITH HIV INFECTION.

\section{1_ Abstract}

\section{Objectives}

Compare the validity of diagnostic criteria for HIV-associated periodontal diseases.

\section{Design}

Cross-sectional.

\section{Method}

Post-hoc application of criteria to data from men with and without HIV.

\section{Outcome measures}

Positive and negative predictive values, sensitivity and specificity for HIV infection.

\section{Results}

Gingival and periodontal ulceration predicted HIV infection to a similar level. Criteria which relied on distinct red bands did not predict HIV.

Criteria sensitive to erythema of the attached gingiva and interdental craters had high positive predictive values.

\section{Conclusions}

Three HIV-associated periodontal changes were recognised: erythema of the attached gingiva; necrotising periodontal disease and reverse architecture/interdental craters. Epidemiological research should also consider conventional gingivitis and lost periodontal attachment.

The presence or absence of all 5 conditions should be recorded at each site. Hierarchies of diagnoses with only the most severe assigned to each individual swamp valuable information. 


\section{$\mathbf{7 . 2}$ Introduction}

A number of diagnostic criteria have been used or proposed for diagnosis of HIVassociated periodontal changes (Greenspan et al, 1992; Laskaris et al, 1992; Masouredis $e t$ al, 1992; Riley et al, 1992; Thompson et al, 1992; EC-Clearinghouse, 1993; Grbic et al, 1995). One system (see Chapter 4) has been shown to improve inter-examiner reliability but none have been validated. The existence of comprehensive data describing the signs and symptoms of periodontal health in homosexual men with and without HIV allows the diagnostic criteria to be tested. Thus, the aim of this study was to assess the validity of existing diagnostic criteria for periodontal changes associated with HIV infection and if necessary to develop more valid criteria.

\section{$7.3 \quad$ Methods}

\subsubsection{Selection of populations}

Data collected in a controlled cross-sectional study of the periodontal health of homosexual men with HIV infection were used (See Chapter 5). These data were considered particularly useful since the sample was large, had been examined blinded to HIV status, was representative of a genito-urinary medicine clinic clientele and was not self-selected for dental disease

\subsubsection{Variables - Summary}

The major variables considered in this study were the criteria. The parameters used therein were compared against variables in the data set to assess the validity of the criteria.

\subsubsection{Selection and development of measures}

\subsubsection{Identification of the criteria}

Original articles were identified by Medline searches of abstracts containing the text words $H I V$ or AIDS and periodontal, periodontitis or gingivitis. The references of all articles identified $w$ ere then checked for other articles, books and book sections not identified in the original search. References of works identified in the second round of searches were also checked for other articles. 
Any paper which had proposed or used diagnostic criteria for periodontal changes associated with HIV was considered. Eight sets of criteria were identified, 5 are presented in Appendices 20 to 24. The sixth set was the criteria developed in Chapter 4 for necrotising ulcerative gingivitis, HIV-associated gingivitis, ulcerative HIV-associated gingivitis, ulcerative HIV-associated periodontitis, non-ulcerative HIV-associated periodontitis, Necrotising periodontitis and attachment loss with reverse architecture. Criteria used by Laskaris et al. (1992) and by Masouredis et al. (1992) were discussed in section 2.3.2.1 and were also considered.

The criteria relate to similar conditions. Those which appear to be equivalent are presented in Table 7.1. The criteria developed in Chapter 4 combined criteria for gingival appearance and attachment levels to define 7 conditions thought to be particularly related to HIV infection. Most other research groups had limited themselves to criteria for linear gingival erythema (previously HIV-G) and necrotising ulcerative periodontitis (previously HIV-P), perhaps with additional criteria for necrotising ulcerative gingivitis (Greenspan et al, 1992; Laskaris et al, 1992; Masouredis et al, 1992; Riley et al, 1992; EC-Clearinghouse, 1993; Grbic et al, 1995). One other group considered only NUG (Thompson et al, 1992).

Table 7.1. Possible equivalents in eight sets of diagnostic criteria of HIV-associated periodontal changes.

\begin{tabular}{|l|l|l|l|l|l|l|l|}
\hline $\begin{array}{l}\text { Riley } \\
\text { (1992) }\end{array}$ & $\begin{array}{l}\text { Thompson } \\
(1992)\end{array}$ & $\begin{array}{l}\text { Masouredis } \\
(1992) .\end{array}$ & $\begin{array}{l}\text { Laskaris } \\
(1992)\end{array}$ & $\begin{array}{l}\text { Greenspan } \\
(1992)\end{array}$ & $\begin{array}{l}\text { EC } \\
(1993)\end{array}$ & $\begin{array}{l}\text { Robinson } \\
(1994)\end{array}$ & $\begin{array}{l}\text { Grbic } \\
(1996)\end{array}$ \\
\hline HIV-G & & HIV-G & HIV-G & HIV-G & LGE & HIV-G & LGE \\
ANUG & NUG & NUG & NUG \\
NUP* & HIV-P & HIV-P & HIV-P & NUP & $\begin{array}{l}\text { HIV-G with } \\
\text { ulceration } \\
\text { HIV-P with } \\
\text { ulceration } \\
\text { Non- } \\
\text { ulcerative } \\
\text { HIV-P } \\
\text { HIV }\end{array}$ & NUP \\
HIV-P & & NUP & & $\begin{array}{l}\text { Necrotising } \\
\text { periodontitis } \\
\text { Reverse } \\
\text { architecture }\end{array}$ & \\
\hline
\end{tabular}

*Although Riley et al. distinguished NUP from HIV-associated periodontitis their descriptions of the two diseases are very similar.

$\mathrm{HIV}-\mathrm{G}=\mathrm{HIV}$-associated gingivitis

LGE = linear gingival erythema

NUP = necrotising ulcerative periodontitis

HIV-P = HIV-associated periodontitis 
Several reports were omitted. Winkler and colleagues have frequently described periodontal conditions seen in patients with HIV but not in the form of criteria (Winkler and Murray, 1987; Winkler et al, 1988; Winkler et al, 1989). This group is represented in two sets of criteria (Masouredis et al, 1992; Chapter 4). Other criteria have related to conventional periodontal diseases (Melnick et al, 1989; Klein et al, 1991; Martínez-Canut et al, 1996). Laskaris et al. (1992) noted that their criterion for HIV-associated periodontitis did not distinguish between this condition and conventional periodontitis. Since this criterion is an extensive description it is difficult to translate it into an explicit test and so it was omitted from the analysis.

\subsubsection{The data set}

Details of the variables collected in this study were presented in Chapter 5. Variables pertinent to this study included symptoms of periodontal diseases, plaque scores, appearance of the marginal and attached gingiva, bleeding on probing, probing attachment loss, pocketing and HIV sero-status. Only data from men of known HIV status were used in the analysis (312 HIV+ve and $260 \mathrm{HIV}$-ve men).

\subsubsection{Periodontal parameters used in the criteria.}

The signs used in the diagnostic criteria were similar but not identical to those used in the cross-sectional study. Some approximations were necessary to apply them post hoc to an existing data set.

Slightly different criteria were used to test the presence of the red bands of linear gingival erythema. For the purposes of analysis all red bands were considered as red bands. Additional criteria for LGE such as differing amounts of bleeding on probing (Masouredis et al, 1992; Chapter 4) or attachment loss (Riley et al, 1992; Chapter 4) were applied as directed.

The EC-Clearinghouse criterion for LGE specifies that the gingival erythema should be disproportionately intense for the amount of plaque. Two more objective translations of this criterion were tested. In the first, only red bands in the absence of visible plaque (plaque score of less than 2) were considered as LGE. The second considered red bands in the absence of a plaque score of 3 (Silness and Löe, 1964) as LGE.

Several criteria for NUG/HIV-P included 'bone pain' (Masouredis et al, 1992; Chapter 4). Data on this symptom were not collected in the questionnaire and the symptom 'painful gums' was substituted instead. 
Criteria which described gingival ulceration limited to the interdental papillae were applied to those where there was less than $4 \mathrm{~mm}$ of lost attachment (Thompson et al, 1992; ECClearinghouse, 1993; Chapter 4).

Two sets of criteria aggregated acute forms of gingival ulceration with any residual defects they may create. The criteria for necrotising ulcerative gingivitis used by Grbic et al. (1995) included ulceration or cratering of the papilla and a history of pain in the last 6 months. Likewise the criteria for both NUG and NUP proposed by the EC-clearinghouse (1993) include acute ulcerative and non-acute forms without ulceration. Only episodes of ulceration current at the time of the examinations were considered. Chapter 4 presents a justification for distinguishing acute ulceration from residual defects for microbiological and other studies and the distinction between prevalent cases and historical cases is sound epidemiological practice (Rothman, 1986).

\subsubsection{Selection of a gold standard}

No gold standard criteria for periodontal diseases were available against which the criteria could be tested. HIV infection was selected as an objective proxy to which the examiner had been blinded. Use of HIV sero-status prevented the calculation of the true sensitivity and specificity of the criteria. Used in this way the sensitivity of a criterion was the apparent prevalence of the condition in HIV infected men and the prevalence in the men without HIV was 1 - specificity.

\subsubsection{Data collection}

Data were collected by applying the criteria to the data set in a personal computer spreadsheet (Excel for the Macintosh Version 4. Microsoft Corp.).

\subsubsection{Data analysis.}

Contingency tables were devised to compare the number of men with and without HIV who met each criterion so that the criteria for equivalent conditions (Table 7.1) were compared by their positive predictive values (PPVs) for HIV infection.

PPVs are dependent on the prevalence of the condition in the population. The probability of a random choice predicting HIV infection is the same as the prevalence of HIV in the sample (prior probability), in this case 0.545 (Galen and Gambino, 1975; Browner et al, 
1988; Altman, 1991). 95\% confidence intervals for the PPVs of different criteria were compared against the prior probability (Galen and Gambino, 1975). The PPVs of individual periodontal measures and groups of measures were also tested.

Confidence intervals were calculated using the Normal approximation for binomial proportions (Altman, 1991). This technique assumes samples of sufficient size (the sample being the men with and without HIV meeting the criteria). When proportions are in the range $0.2-0.8$ samples as small as 25 may be used. At outside this range samples of 50 are required. Confidence intervals were not calculated for samples of inadequate size.

\subsection{Results}

Table 7.2 presents the predictive values, sensitivity, specificity and 1 - specificity for criteria for ulceration limited to the marginal gingiva. All measures are identical for the criteria of Thompson et al. (1992), the EC-Clearinghouse (1993) and Grbic et al. (1995) and it can be seen in Tables 7.2, 7.4 and 7.5 that these criteria have similar face validity if non-ulcerative craters are excluded. There were not sufficient cases of ulceration to allow calculation of confidence intervals for gingival or more extensive ulceration.

Table 7.3. summarises the same measures for criteria relating to more extensive ulceration. Several criteria for HIV-P and NUP (Masouredis et al, 1992; Chapter 4; EC-Clearinghouse, 1993; Grbic et al, 1995) had PPVs of 1 demonstrating that cases were limited to the men with HIV but there were too few cases to estimate confidence intervals for any values.

Table 7.4 presents the measures for criteria for LGE (previously HIV-G). Two had confidence intervals which did not include the prior probability and so predicted HIV infection. These were the criteria by Masouredis et al. (1992) and in Chapter 4. The PPVs of the component parts of criteria for LGE/HIV-G were subjected to the same analyses (Table 7.5). Only diffuse erythema and diffuse and/or punctate erythema predicted HIV infection.

The ability of patterns of attachment loss to predict HIV infection are presented in Table 7.6. There were too few men with non-ulcerative HIV-P to estimate a confidence interval and a similar pattern of attachment loss in the absence of unusual gingivitis did not predict HIV infection. However, this pattern of attachment loss predicted HIV infection if considered regardless of the colour of the gingiva. 
The ability of areas of attachment loss with minimal pocketing to predict HIV was also assessed for all sites, interdental sites and buccal only sites. This analysis was adjusted to exclude the effect of greater prevalence of attachment loss in the men with HIV by excluding men who did not have attachment loss of $4 \mathrm{~mm}$ or more. 187 men with HIV and 75 without had attachment loss resulting in a prior probability of 0.714 . Only attachment loss in interdental sites predicted HIV infection (Table 7.7).

Table 7.2. Validity of 5 diagnostic criteria for ulceration of the marginal gingiva associated with HIV infection

\begin{tabular}{|l|ccccc|}
\hline & PPV & NPV & Sensitivity & Specificity & 1 - specificity \\
\hline Riley (1992) & 1.000 & 0.456 & 0.006 & 1.000 & 0 \\
Thompson (1992) & 0.600 & 0.455 & 0.035 & 0.985 & 0.015 \\
EC (1993) & 0.60 & 0.455 & 0.035 & 0.985 & 0.015 \\
Chapter 4 NUG & 0.667 & 0.455 & 0.006 & 0.985 & 0.015 \\
Chapter 4 & 0.570 & 0.455 & 0.013 & 0.973 & 0.027 \\
HIV-G with ulceration & & & & & \\
Grbic (1995) & 0.600 & 0.455 & 0.035 & 0.985 & 0.015 \\
\hline
\end{tabular}

PPV $=$ Positive predictive value

NPV $=$ Negative predictive value

Only 1 men had gingival ulceration and confidence intervals for the PPV could not be calculated. 
Table 73. Validity of 5 diagnostic criteria for extensive periodontal ulceration associated with HIV infection.

\begin{tabular}{|l|ccccc|}
\hline & PPV & NPV & Sensitivity & Specificity & 1 - specificity \\
\hline Riley (1992) & 0.667 & 0.457 & 0.026 & 0.985 & 0.015 \\
Masouredis (1992) & 1.000 & 0.457 & 0.010 & 1.000 & 0 \\
EC (1993) & 1.000 & 0.458 & 0.013 & 1.000 & 0 \\
Chapter 4 & 1.000 & 0.457 & 0.010 & 1.000 & 0 \\
Grbic (1995) & 1.000 & 0.458 & 0.013 & 1.000 & 0 \\
\hline
\end{tabular}

PPV $=$ Positive predictive value

$\mathrm{NPV}=$ Negative predictive value

Table 7.4. Validity of 5 diagnostic criteria for linear gingival erythema/HIVassociated gingivitis.

\begin{tabular}{|l|lcccc|}
\hline & PPV $(95 \%$ CI $)$ & NPV & Sensitivity & Specificity & 1 - specificity \\
\hline Riley (199; $)$ & $\begin{array}{l}0.458 \\
(0.265-0.651)\end{array}$ & 0.451 & 0.122 & 0.950 & 0.050 \\
Masouredis (1992) & $\begin{array}{l}0.705 \\
(0.624-0.789)^{*}\end{array}$ & 0.498 & 0.276 & 0.861 & 0.139 \\
EC (1993) (PS < 2) $\dagger$ & $\begin{array}{l}0.750 \\
\text { (N/A) }\end{array}$ & 0.456 & 0.010 & 0.996 & 0.004 \\
EC (1993) (PS < 3) $\dagger$ & $\begin{array}{l}0.653 \\
(0.471-0.835)\end{array}$ & 0.459 & 0.054 & 0.965 & 0.035 \\
Chapter 4 & $\begin{array}{l}0.758 \\
(0.648-0.868) *\end{array}$ & 0.478 & 0.141 & 0.946 & 0.054 \\
Grbic (1995) & $\begin{array}{l}0.593 \\
(0.473-0.713)\end{array}$ & 0.539 & 0.122 & 0.900 & 0.100 \\
\hline
\end{tabular}

PPV $=$ Positive predictive value

$\mathrm{NPV}=$ Negative predictive value

$\mathrm{CI}=$ Confidence interval

$\uparrow$ Two possible of the EC criteria were tested depending on the amount of plaque present. N/A Sample size was too small to permit calculation of confidence intervals.

* $95 \%$ CI does not include prior probability. 
Table 7.5. Validity of 6 signs of linear gingival erythema/HIV-associated gingivitis.

\begin{tabular}{|l|lcccc|}
\hline & PPV (95\% CI) & NPV & Sensitivity & Specificity & 1 - specificity \\
\hline Any red bands & $\begin{array}{l}0.594 \\
(0.470-0.610)\end{array}$ & 0.460 & 0.122 & 0.900 & 0.100 \\
$\begin{array}{l}\text { Red bands with bleeding } \\
<50 \% \text { sites }\end{array}$ & $\begin{array}{l}0.600 \\
(0.360-0.840)\end{array}$ & 0.456 & 0.029 & 0.977 & 0.023 \\
$\begin{array}{l}\text { Diffuse erythema attached } \\
\text { gingiva }\end{array}$ & $\begin{array}{l}0.830 \\
(0.722-0.937)^{*}\end{array}$ & 0.480 & 0.125 & 0.969 & 0.031 \\
$\begin{array}{l}\text { Punctate erythema attached } \\
\text { gingiva }\end{array}$ & $\begin{array}{l}0.909 \\
(\mathrm{~N} / \mathrm{A})\end{array}$ & 0.477 & 0.096 & 0.988 & 0.012 \\
$\begin{array}{l}\text { Diffuse and/or Punctate } \\
\text { erythema }\end{array}$ & $\begin{array}{l}0.864 \\
(0.775-0.952)^{*}\end{array}$ & 0.496 & 0.183 & 0.965 & 0.035 \\
\hline
\end{tabular}

PPV = Positive predictive value

NPV $=$ Negative predictive value

$\mathrm{CI}=$ Confidence interval

* 95\% CI does not include prior probability.

Table 7.6. Validity of 3 tests of lost periodontal attachment associated with HIV infection (Crude data).

\begin{tabular}{|l|lcccc|}
\hline & PPV $(95 \%$ CI $)$ & NPV & Sensitivity & Specificity & 1 - specificity \\
\hline $\begin{array}{l}\text { Chapter 4 } \\
\text { Non-Ulc HIV-P }\end{array}$ & $\begin{array}{l}1.000 \\
\text { N/A) }\end{array}$ & 0.471 & 0.067 & 1.000 & 0 \\
$\begin{array}{l}\text { Chapter 4 } \\
\text { Reverse architecture/Craters }\end{array}$ & $\begin{array}{l}0.598 \\
(0.504-0.682)\end{array}$ & 0.470 & 0.253 & 0.796 & 0.204 \\
$\begin{array}{l}\text { Reverse architecture } \\
\text { Craters } \pm \text { HIV-G }\end{array}$ & $\begin{array}{l}0.653 \\
(0.578-0.729) *\end{array}$ & 0.494 & 0.320 & 0.796 & 0.204 \\
\hline
\end{tabular}

PPV $=$ Positive predictive value

NPV $=$ Negative predictive value

$\mathrm{CI}=$ Confidence interval

Non-Ulc HIV-P = Non-ulcerative HIV-Periodontitis

* 95\% CI does not include prior probability. 
Table 7.7. Validity at sites with lost periodontal attachment in HIV infection (Adjusted data)

\begin{tabular}{|c|c|c|c|c|c|}
\hline & PPV $(95 \% \mathrm{CI})$ & NPV & Sensitivity & Specificity & 1 - specificity \\
\hline Any site & $\begin{array}{l}0.725 \\
(0.666-0.784)\end{array}$ & 0.304 & 0.620 & $\overline{0.413}$ & 0.587 \\
\hline Mesial and Distal sites & $\begin{array}{l}0.806 \\
(0.740-0.872)^{*}\end{array}$ & 0.313 & 0.524 & 0.632 & 0.368 \\
\hline Buccal sites & $\begin{array}{l}0.685 \\
(0.606-0.764)\end{array}$ & 0.300 & 0.643 & 0.341 & 0.659 \\
\hline
\end{tabular}

* $95 \%$ CI does not include prior probability

\section{$7.5 \quad$ Discussion}

This study aimed to validate diagnostic criteria for periodontal changes associated with HIV infection by measuring their ability to predict which of 572 homosexual men had HIV. Seven sets of explicit and objective criteria were identified.

Only 15 men had any gingival or periodontal ulceration, so criteria relating these conditions could not be validated. Three criteria for gingival ulceration had similar face validity and identical PPVs, NPVs, sensitivity and specificity (Thompson et al, 1992; ECClearinghouse, 1993; Grbic et al, 1995)(Table 7.2). Two of the remaining three criteria (Masouredis et al, 1992 and those in Chapter 4) distinguished the appearance of the adjacent gingiva and so were less sensitive, although cumulatively they would correspond to the previous three. The Riley criterion for NUG (Riley et al, 1992) specifies there should be no loss of bone and detects fewer cases than those which accept up to $3 \mathrm{~mm}$ lost attachment.

Most criteria for severe ulceration (NUP/HIV-P) had PPVs of 1 and similar values for the other measures of validity (Table 7.3). Although the criteria used by Masouredis et al. (1992), the EC-Clearinghouse (1993), Grbic et al. (1995) and in Chapter 4 appear different they are operationally similar if evidence of previous ulceration is ignored. In the sample, ulceration more than $3 \mathrm{~mm}$ from the CEJ was always accompanied by craters, minimal pocketing and pain so these additional criteria were superfluous. Both the Chapter 4 and Masouredis criteria for HIV-P are slightly less sensitive because they make stipulations about the appearance of the adjacent gingiva. 
The very stringent criterion for NUG used by Riley et al. (1992) is reflected in the larger number of cases of NUP. This arbitrary distinction between NUG and NUP and the findings of Chapter 6 suggest that the two need not be distinguished. Another way of recording the severity of ulceration is required.

There were sufficient cases of LGE/HIV-G to attempt statistical validation of the criteria (Table 7.4). The two criteria which predicted HIV infection were sensitive to diffuse or punctate erythema of the attached gingiva (Masouredis et al, 1992 and Chapter 4). Neither criteria which relied on red bands nor the bands themselves predicted HIV infection (although there were too few cases of red bands with low plaque scores to validate the ECClearinghouse (1993) criterion fully)(Table 7.5). This is a surprising finding as HIVG/LGE are frequent findings in several studies. (Klein et al, 1991; Masouredis et al, 1992; Riley et al, 1992). However, neither the study from which this data is drawn, nor the similar study by Grbic et al. (1995) associated gingival red bands with HIV infection. These studies remain the largest and most comprehensive controlled studies to date.

This indicates that criteria for LGE which relate to red bands are not useful. The disease is uncommon and more specific tests are required at low prevalences to prevent excess false positive diagnoses (Galen and Gambino, 1975; Browner et al, 1988; Altman, 1991). The resistance of LGE to plaque removal over sequential visits is the only criterion available with any validity. Both the EC-clearinghouse (1993) and the US collaborative group include this in their definitive criteria and others should not be used (Greenspan et al, 1992).

Diffuse erythema of the attached gingiva predicted HIV infection. There were too few cases of punctate erythema to estimate a confidence interval, but aggregating this condition with diffuse erythema produced a more predictive test with a narrower confidence interval. Diagnostic criteria for HIV-associated periodontal changes should be sensitive to these conditions and there does not seem to be any justification for aggregating them with the red band form of LGE. The relationship between erythema of the attached gingiva, HIV infection and oral candidiasis demonstrated in Chapter 5 and in Appendix 15 suggests that erythema of the attached gingiva is gingival candidiasis.

Reverse architecture or cratering also predicted HIV infection (although when this pattern was divided into those with and without 'HIV-gingivitis' the analysis lost power)(Table 7.6). These criteria detect cases with $4 \mathrm{~mm}$ lost attachment where the recession is more than twice the pocket depth, so their ability to predict HIV may reflect the increased prevalence 
of attachment loss among infected men. In an analysis adjusted to exclude the effect of attachment loss prevalence, interdental craters significantly predicted HIV whereas attachment loss at buccal sites did not (Table 7.7). It is plausible that interdental craters are the legacy of ulcerative disease although their prevalence among men with HIV infection (32\%, Table 7.6) is higher than would be expected from the 1 to $2 \%$ annual incidence reported in Chapter 6. This also suggests that criteria should not group ulcerative disease with non-ulcerative defects, but that the two should be recorded independently.

Several of the criteria consider the colour of the gingiva in tandem with ulceration or lost attachment (Masouredis et al, 1992 and Chapter 4). These are aggregate indices of the kind criticised by Burt (1991). The stipulations for gingival colour in the criteria for HIV-G with ulceration and HIV-P do not add to the usefulness of the criteria and could be removed to aid simplicity.

Erythema of the attached gingiva should not be disregarded, it should be disaggregated. If erythema of the attached gingiva is gingival candidiasis it is of some interest. Candida spp. are found in the plaque of people with HIV (Murray et al, 1989; Zambon et al, 1990), and oral candidiasis is associated with various forms of gingivitis and necrotising ulcerative gingivitis/periodontitis in the same group of people (Odden et al, 1994; Grbic et al, 1995 and Chapters 5 and 6). Since diffuse and punctate erythema are similarly associated with HIV infection and candidiasis there does not seem to be any justification for considering the two forms separately.

New diagnostic criteria are required for HIV-associated periodontal changes. Three conditions predict HIV infection: erythema of the attached gingiva; necrotising periodontal disease and reverse architecture/interdental craters. Suitable criteria, based on the findings of this study and the reliability study in Chapter 4 are suggested in Table 7.8. The crosssectional study reported in Chapter 5 also associated conventional gingivitis and lost attachment (of $4 \mathrm{~mm}$ or more) with HIV infection, thus future epidemiological research should consider these conditions too.

The most useful method would be to record which of the 5 conditions are present at each site. Recording both attachment loss and ulceration in this way would measure the severity of ulceration. More information is gained if erythema of the attached gingiva is noted as present or absent at the same site. Creating a hierarchy of diagnoses with only the most severe assigned to each individual may swamp valuable information (Riley et al, 1992). The diagnoses describe different characteristics and need not be mutually exclusive with one exception: attachment loss without interdental craters and interdental cratering cannot be present at the same site. 
These new criteria need to be validated in other samples (Browner et al, 1988). This study was based on data collected from homosexual men in London, UK. Differences in the presentation of HIV-associated periodontal changes have been attributed to geographical differences, route of HIV transmission and age (Winkler et al, 1989; San Martin et al, 1992; Grbic et al, 1995). Future validation studies could be undertaken in all of these groups, not only to validate the criteria but also to compare the periodontal health of the different groups.

Table 7.8. Operational criteria for periodontal changes associated with HIV infection

Erythema of the attached gingiva

Defined by the presence of one of the following:

i) punctate erythema of the attached gingiva

ii) diffuse erythema of the attached gingiva

Necrotising periodontal disease (NUG/NUP)

Defined by the presence of ulceration of one or more interdental papillae

Attachment loss with reverse architecture or inter-dental cratering

Defined by the presence of attachment loss $\geq 4 \mathrm{~mm}$ and where the recession is $\geq 2 \mathrm{x}$ the probing depth (excluding the mid-buccal surfaces).

\subsection{Conclusions}

Seven sets of diagnostic criteria for HIV-associated periodontal changes were identified and tested against data collected from homosexual men with and without HIV. Three conditions predicted HIV infection: erythema of the attached gingiva; necrotising periodontal disease and reverse architecture/interdental craters.

Criteria which relied on the presence of gingival ulceration had similar validity and additional conditions within these criteria were unnecessary.

Criteria for gingival changes which were sensitive to erythema of the attached gingiva had greater validity than those dependent on bright red bands. Presumptive criteria for linear gingival erythema are not useful in adults. 
In HIV disease, more lost attachment is manifest as interdental defects with shallow pockets. Recurrent episodes of ulcerative disease are at least partly responsible for periodontal destruction in HIV disease but do not seem to be sufficiently common to account for all of the lost attachment in infected people.

A simple diagnostic system would be to record the presence or absence of erythema of the attached gingiva; necrotising periodontal disease, reverse architecture/interdental craters, conventional gingivitis and lost attachment at each site. Except for distinguishing attachment with or without interdental defects, diagnoses are neither mutually exclusive nor hierarchical. 


\section{CHAPTER 8 CONCLUSIONS AND RECOMMENDATIONS FOR FUTURE RESEARCH}

The conclusions drawn from this research relate directly to periodontal health or to diagnosis of disease.

\subsection{Periodontal health and HIV infection}

1. Several periodontal changes are associated with HIV infection: the prevalence extent and severity of probing attachment loss, pocketing and conventional forms of gingivitis. These relationships are independent of age, plaque and tobacco use.

2. Gingival ulceration, bleeding on probing and diffuse and punctate erythema of the attached gingiva are also more common in HIV infection.

3. Bright red bands ('LGE') were not associated with HIV infection in this population.

4. Lost periodontal attachment is more common, extensive and severe in advanced HIV disease. Periodontal pocketing does not increase with advanced HIV disease.

5. Oral candidiasis is a risk factor for conventional gingivitis, erythema of the attached gingiva and gingival ulceration.

6. The prevalence of necrotising periodontal disease among men with HIV is less than $3.2 \%$. The incidence is approximately $1.5 \%$.

7. The most common form of gingival ulceration seen in HIV infection could not be distinguished from aggressive ANUG.

8. Relapse of ANUG is common. There may be a high risk group for the disease.

9. ANUG in HIV infection is associated with oral candidiasis, lower age and early HIV infection. Co-trimoxazole is protective.

10. Some ulceration resembles the Ulceration NOS of HIV infection but is associated with neutropenia. 
8.2 The diagnosis of periodontal changes seen in HIV infection

11. A group of experienced examiners were only fairly reliable when recognising signs and making diagnoses of periodontal conditions.

12. Diagnoses were intuitive and did not correspond with descriptions of the changes.

13. These findings significantly undermine earlier research of these changes.

14. Rigid diagnostic criteria, training and calibration increase the value of studies of HIV-associated periodontal changes.

15. The most valid diagnostic criteria for HIV-associated changes were sensitive to erythema of the attached gingiva, necrotising periodontal disease and lost interdental attachment.

16. The presence of conventional gingivitis and lost attachment should also be recorded in studies of periodontal health in HIV infection.

17. The presence or absence of these conditions should be recorded at individual sites. Diagnoses are neither mutually exclusive nor hierarchical.

18. Criteria dependent on bright red bands were invalid. Presumptive criteria for LGE are not useful in adults.

19. Recurrent episodes of ulcerative disease are partly responsible for the pattern of periodontal destruction in HIV disease but may not account for all of the lost attachment. 


\subsection{Recommendations for future work}

Much of the research reported here is descriptive and exploratory. It is hypothesis generation, not proof of cause and effect. Therefore several avenues for future research are available including longitudinal studies, intervention studies, laboratory research, validation studies and comparison of disease in different groups.

\subsubsection{Longitudinal research}

Longitudinal studies are the gold standard in periodontology and are essential to confirm the findings of the cross-sectional studies.

Cohort studies could confirm that HIV and oral candidiasis are risk factors for the above changes. Such studies will need follow-up periods of at least 2 years. Frequent simple reviews would allow investigation of the role of candidiasis in the aetiology of ulcerative disease and relate the incidence of ulceration and other gingival changes to attachment loss.

The periodontal destruction anticipated in people with HIV as a result of this research suggests that positive findings are more likely than in other avenues of periodontology.

\subsubsection{Intervention studies}

The high relapse rate of ulcerative disease demands the development of interventions to prevent this disease. The regimen reported here are suitable controls for randomised trials. The regimen used in the acute phase may be the most effective and efficient but the preventive approach was not successful.

The effect of antifungals on the gingival disease should be studied to assess the role of candidiasis in the aetiology of these diseases.

\subsubsection{Validation studies}

Studies with HIV negative controls are required to validate the diagnostic criteria proposed in Chapter 7. Such studies should only be undertaken after calibration of examiners. If the criteria are valid they can be used as a gold standard for future criteria. 


\subsubsection{Laboratory research}

Microbiological studies are needed to investigate the role of Candida spp. in the pathogenesis of the periodontal changes described earlier. Similarly, the role of fusiforms and spirochaetes in the pathogenesis of ulcerative disease could be studied, perhaps with electron microscopy. These studies would need to be controlled with samplesfrom sites with defects but without ulceration in people with HIV as well sites with ANUG in known HIV negative people.

Laboratory research must be related to valid clinical descriptions of the sites sampled. The criteria proposed above may be the most suitalle. However, any explicit and unambiguous criteria are better than none.

\subsubsection{Periodontal health in different groups}

This research was concerned primarily with HIV disease in homosexual men in London, England. The variation in findings of earlier research has been attributed to geographical differences and differences between homosexual men and drug users with HIV. Studies similar to the ones reported or suggested here should be used to compare the periodontal health of people with HIV in different transmission groups and different locations. Diagnostic criteria must also be validated in these groups.

\subsubsection{Relationship to conventional diseases}

It is too early to know how much HIV-associated periodontal changes inform us about periodontal diseases in the general population. If Candida spp. and more frequent gingival ulceration are implicated in periodontal destruction then the knowledge gained from HIV infection is not directly applicable to conventional periodontal diseases.

On the other hand, accelerated periodontal destruction in HIV disease may be a suitable model for other periodontal research, particularly that into the pathogenesis. Those susceptible to gingival ulceration are a 'high risk group' for periodontal attachment loss and may warrant further study. 
Collaboration is not a research method but is an essential strategy. Some of the periodontal changes associated with HIV are uncommon. Classical linear gingival erythema is an obvious example, but gingival ulceration was not sufficiently common to allow sophisticated analysis of aetiological factors. Collaboration will aid investigation of these conditions in cross-sectional or longitudinal research and is essential for controlled studies of management regimen. Case-control studies are well suited to studying rare outcomes.

Collaborative research demands standardised data collection between centres and the problems of random allocation to treatment groups are much greater in multi-centre trials. Despite this, collaboration is required if our knowledge of HIV-associated periodontal changes is to increase. 


\section{REFERENCES}

Abdellatif HM, Burt BA (1987). An epidemiological investigation into the relative importance of age and oral hygiene status as determinants of periodontitis. $J$ Dent Res 66: 13-8.

Abel SN, Andriolo M (1989). Clinical management of HIV-related periodontitis: report of a case. J Am Dent Assoc 119, Supplement; 35S-36S.

Ainamo J, Barmes D, Beagrie G, Cutress T, Martin J, Sardo-Infirri J (1982). Development of the World Health Organisation (WHO) Community Periodontal Index of Treatment Needs (CPITN). Int Dent J. 132: 281-91.

Akula SK, Creticos CM, Weldon-Linne CM (1989). Gangrenous Stomatitis in AIDS (Letter). Lancet 1: 955.

Albander JM, Goldstein H (1992). Multi-level statistical models in studies of periodontal diseases. J Periodontol 63: 690-5.

Alexander AG, Leon AR, Ribbons JW, Morganstein SI (1971). An assessment of the interand intra-examiner agreement in scoring gingivitis clinically. $J$ Periodont Res 6: 146-51.

Altman DG (1991). Practical statistics for medical research. Chapman and Hall, London.

Apaiza T, Franco F, Pebollo J (1991). Periodontal status and subgingival microflora in HIV (+) patients. Clinical report. (Abstract). J Dent Res 79: 415.

Armitage GC, Svanberg GK, Löe H (1977). Microscopic evaluation of clinical measurements of connective tissue attachment levels. J Clin Periodontol 4: 173-90.

Axelsson P, Lindhe J (1978). Effect of controlled oral hygiene procedures on caries and periodontal disease in adults. $J$ Clin Periodontol 5: 133-51.

Badersten A, Nilveus R, Egelberg J (1984). Reproducibility of probing attachment level measurements. J Clin Periodontol 11: 475-85.

Baehni PC, Payot P, Tsai C-C, Cimasoni G (1983). Periodontal status associated with chronic neutropenia. J Clin Periodontol 10: 222-30. 
Baelum V, Manji F, Fejerskov O (1990). A new approach to investigating associations in periodontal disease data. Community Dent Oral Epidemiol 18: 177-83.

Baelum V, Manji F, Wanzala P, Fejerskov O (1995). Relationship between CPITN and periodontal attachment loss findings in an adult population. J Clin Periodontol 22: 146-52.

Baker EG, Crook GH, Schwabacher ED (1961). Personality correlates of periodontal disease. J Dent Res 40: 396-403.

Ballieux RE (1991). Impact of mental stress on the immune response. J Clin Periodontol 18: 427-30.

Barnes GP, Bowles WF, Carter HG (1973). Acute necrotising ulcerative gingivitis: a survey of 218 cases. J Periodontol 44: 35-42.

Barnes GP, Parker WA, Lyon TC, Fultz RP (1986). Indices used to evaluate signs, symptoms and etiologic factors associated with diseases of the periodontium. $J$ Periodontol 56: 643-51.

Barr C, Lopez MR, Rua-Dobles A (1992). Periodontal changes by HIV serostatus in a cohort of homosexual and bisexual men. J Clin Periodontol 19: 794-801.

Been V, Engel D (1982). The effects of immunosuppressive drugs on periodontal inflammation in human renal allograft patients. J Periodontol 53: 245-8.

Belting CM, Gupta OP (1960). Incidence of periodontal disease among persons with neuropsychiatric disorders. J Dent Res 39: 744-5.

Bentley CD, Disney JA (1995). A comparison of partial and full mouth scoring of plaque and gingivitis in oral hygiene studies. J Clin Periodontol 22: 131-5.

Beral V, Peterman TA, Berkelman RL, Jaffe HW (1990). Kaposi's sarcoma among persons with AIDS: a sexually transmitted infection? Lancet 335: 123-8.

Bergstrom J (1989). Cigarette smoking as a risk factor in chronic periodontal disease. Community Dent Oral Epidemiol 17: 245-7.

Bergstrom J, Eliasson S, Preber H (1991). Cigarette smoking and periodontal bone loss. $J$ Periodontol 62: 242-6. 
Brown LJ, Oliver RC, Löe H (1989). Periodontal diseases in the US. in 1981: Prevalence, severity, extent, and role in tooth mortality. J Periodontol 60: 363-70.

Browner WS, Newman TB, Cummings SR (1988). Designing a new study: 3. Diagnostic tests. In: Hulley SB, Cummings SR, Eds. Designing Clinical Research. Williams and Wilkins. Baltimore, pp 87-97.

Burt BA (1991). The distribution of periodontal destruction in the populations of industrialised countries. In: Johnson NW, Ed. Risk markers for oral diseases. Volume 3: Periodontal diseases: Markers of disease susceptibility and activity. Cambridge University Press. Cambridge, pp 9-26.

Caton J (1989). Periodontal diagnosis and diagnostic aids. World Workshop in Clinical Periodontics, Princeton, American Academy of Periodontology.

Centers for Disease Control and Prevention (1992). 1993 revised classification system for HIV infection and expanded surveillance case definition for AIDS among adolescents and adults. $M M W R$ 41: 1-19.

Centers for Disease Control and Prevention (1992). Guidelines for prophylaxis against Pneumocystis carinii pneumonia for persons infected with human immunodeficiency virus. MMWR 41: 1-11.

Centre for Disease Surveillance and Control (1995). AIDS and HIV-1 infection in the United Kingdom: monthly report. CDR 5: 217-20.

Centre for Disease Surveillance and Control (1996). AIDS and HIV-1 infection in the United Kingdom: monthly report. CDR 6: 99-100.

Challacombe SJ, Schiodt M (1995). HIV-associated salivary gland disease. In: de Burgh Norman JE, McGurk M, Eds. Color atlas and text of the salivary glands. Diseases, disorders and surgery. Mosby-Wolfe. London, pp 279-83.

Chang Y, Cesarman E, Pessin M, Lee F, Culpepper J, Knowles DM, Moore PS (1994). Identification of Herpesvirus-like DNA sequences in AIDS associated Kaposi's sarcoma. Science 266: 1865-9.

Chapple IL, Rout PG, Basu MK (1992). Gingival Kaposi's sarcoma: the first indication of HIV infection. Dental Update 19: 296-301. 
Charon JA, Mergenhagen SE, Gallin JI (1985). Gingivitis and oral ulceration in patients with neutrophil dysfunction. J Oral Pathol 14: $150-5$.

Christersson LA, Zambon JJ, Genco RJ (1991). Dental bacterial plaques. Nature and role in periodontal disease. J Clin Periodontol 18: 441-6.

Cianciola LJ, Park BH, Bruck E, Mosovich L, Genco RJ (1982). Prevalence of periodontal disease in insulin dependent diabetes mellitus (juvenile diabetes). J Am Dent Assoc. 104: 653-60.

Claffey N, Russell R, Shanley D (1986). Peripheral blood phagocyte function in acute necrotising ulcerative gingivitis. J Periodont Res. 21: 288-97.

Clarke NG, Hirsch RS (1995). Personal risk factors for generalized periodontitis. $J$ Clin Periodontol 22: 136-45.

Cogen RB, Stevens AW, Cohen-Cole S, Kirk K, Freeman A (1983). Leukocyte function in the etiology of acute necrotising ulcerative gingivitis. J Periodontol 54: 402-7.

Cohen DW, Morris AL (1961). Periodontal manifestations of cyclic neutropenia. $J$ Periodontol 32: 159-68.

Cohen-Cole SA, Cogen RB, Stevens AWJ, Kirk K, Gaitan E, Bird J, Cooksey R, Freeman A (1983). Psychiatric, psychosocial, and endochrine correlates of acute necrotizing ulcerative gingivitis (Trench mouth): a preliminary report. Psychiatric Med 1: 215-25.

Colebunders R, Francis H, Mann JM, Bila KM, Kandi K, Lebughe I, Gigase P, Van Marck E, Macher AM, Quinn TC (1988). Parotid swelling during human immunodeficiency virus infection. Arch Otolaryngol Head Neck Surg. 114: 330-2.

Connor RI, Ho DD (1992). Etiology of AIDS: Biology of human retroviruses. In: DeVita VT, Hellman S, Rosenberg SA, Eds. AIDS. Etiology, diagnosis, treatment and prevention. J.B. Lippincott Company. Philadelphia, pp 13-38.

Cornick DER, Robinson PG (1991). A three year study of the periodontal condition in HIV positive men (Abstract). J Dent Res 70: 688.

Courtois GJ, Cobb CM, Killoy WJ (1983). Acute necrotising ulcerative gingivitis: a transmission electron microscope study. J Periodontol 54: 671-9. 
Cowell CR, Saxton CA, Sheiham A, Wagg BJ (1975). Testing therapeutic measures for controlling chronic gingivitis in man: a suggested protocol. J Clin Periodontol 2: 231-40.

Cox T (1978). Stress. Macmillan Education Ltd., Basingstoke.

Cross DL, Smith GLF (1995). Comparison of periodontal disease in HIV seropositive subjects and controls (II). Microbiology, immunology and predictors of disease progression. J Clin Periodontol 22: 569-77.

Cuff MJA, McQuade MJ, Scheidt MJ, Sutherland DE, Van Dyke TE (1989). The presence of nicotine on root surfaces of periodontally diseased teeth in smokers. $J$ Periodontol 60: 564-9.

Datta M (1993). You cannot exclude the explanation you have not considered. Lancet 342: 345-7.

Davey-Smith G, Phillips AN (1992). Confounding in epidemiological studies: Why 'independent' effects may not be all they seem. Br Med J 305: 757-9.

Deasy MJ, Vogel RI, Macedo-Sobrinho B, Gertzman G, Simon B (1980). Familial benign chronic neutropenia associated with periodontal disease. A case report. J Periodontol 51: 206-10.

Dennison DK, Smith B, Newland JR (1985). Immune responsiveness and ANUG (Abstract). J Dent Res 64: 197.

DeRouen TA, Mancl L, Hujoel P (1991). Measurement of associations in periodontal diseases using statistical methods for dependent data. J Periodont Res 26: 218-29.

Dodd CL, Greenspan D, Katz MH, Westenhouse JL, Feigal DW, Greenspan JS (1991). Oral candidiasis in HIV infection: pseudomembranous and erythematous candidiasis show similar rates of progression to AIDS. AIDS 5: 1339-43.

Dodd CL, Winkler JR, Heinic GS, Daniels TE, Yee K, Greenspan D (1993). Cytomegalovirus infection presenting as acute periodontal infection in a patient with the human immunodeficiency virus. J Clin Periodontol 20: 282-5. 
Drinkard CR, Decher L, Little JW, Rhame FS, Balfour HH Jr., Rhodus NL, Merry JW, Walker PO, Miller CE, Volberding PA, Melnick SL (1991). Periodontal status of individuals in early stages of human immunodeficiency virus infection. Community Dent Oral Epidemiol 19: 281-5.

Ducka-Karsa K (1979). Affections bucco-dentaires dans la population de Lubum-bashi (Zaire). Trop Dent J 8: 15-17.

EC-Clearinghouse on oral problems related to HIV infection and WHO Collaborating Centre on oral manifestations of the immunodeficiency virus (1993). Classification and diagnostic criteria for oral lesions in HIV infection. J Oral Pathol Med 22: 289-91

EEC-Clearinghouse on oral problems related to HIV infection and WHO Collaborating Centre on oral manifestations of the Human Immunodeficiency Virus (1991). An update of the classification and diagnostic criteria of oral lesions in HIV infection. J Oral Pathol Med 20: $97-100$.

Elbim C, Prevot MH, Bouscarat F, Franzini E, Chollet-Martin S, Hakim J, GougerotPocidato MA (1994). Polymorphonuclear neutrophils from human immunodeficiency virusinfected patients show enhanced activation, diminished fMLP-induced L-selectin shedding, and an impaired oxidative burst after cytokine priming. Blood 84: 2759-66.

Ellis M, Gupta S, Galant S, Hakim S, VandeVen C, Toy C, Cairo MS (1988). Impaired neutrophil function in patients with AIDS or AIDS-related complex. A comprehensive evaluation. $J$ Infect Dis 158: 1268-76.

Emslie RD (1963). Cancrum oris. Dent Practitioner 13: 481-95.

Epstein JB, Leziy S, Ransier A (1993). Periodontal pocketing in HIV-associated periodontitis. Spec Care Dent 13: 236-40.

Epstein JB, Silverman SJ (1992). Head and neck malignancies associated with HIV infection. Oral Surg Oral Med Oral Pathol 73: 193-200.

Espeland MA, Zappa UE, Hogan PE, Simona C, Graf H (1991). Cross-sectional and longitudinal reliability for clinical measurement of attachment loss. $J$ Clin Periodontol 18: 126-33. 
European Collaborative Study (1991). Children born to women with HIV-1 infection: natural history and risk of transmission. Lancet 337: 253-60.

Fahey JL, Taylor JMG, Detels R, Hofmann B, Melmed R, Nishanian P, Giorgi JV (1990). The prognostic value of cellular and serologic markers in infection with human immunodeficiency virus type 1. NEngl J Med 322: 166-72.

Farthing C (1986). AIDS, The Dental Picture. Dental Practice 24: 1-2.

Farthing C, Brown S, Staughton R (1988). A colour atlas of AIDS and HIV disease. Wolfe Medical Publications Ltd, London.

Fedi PF (1958). The effects of stress in the periodontium of the syrian hamster. $J$ Periodontol 29: 292-9.

Felix DH, Wray D, Smith GLF, Jones GA (1991). Oro-antral fistula: an unusual complication of HIV-associated periodontal disease. Br Dent J 171: 61-2.

Flø RW, Naess A, Nilsen A, Harthug S, Solberg CO (1994). A longitudinal study of phagocyte function in HIV-infected patients. AIDS 8: 771-7.

Fox PC (1992). Salivary gland involvement in HIV-1 infection. Oral Surg Oral Med Oral Pathol 73: 168-70.

Friedman RB, Gunsolley J, Gentry A, Dinius A, Kaplowitz L, Settle J (1991). Periodontal status of HIV-seropositive and AIDS patients. J Periodontol 62: 623-27.

Galen RS, Gambino SR (1975). Beyond normality. The predictive value and efficiency of medical diagnoses. John Wiley \& Sons, New York.

Garfunkel AA, Glick M (1995). Similarities between oral lesions in AIDS and other immunodeficiency states. In: Greenspan JS, Greenspan D, Eds. Oral Manifestations of HIV Infection. Quintessence Publishing Co, Inc. Chicago, pp 218-124.

Genco RJ (1990). Pathogenesis and host responses in periodontal disease. In: Genco RJ, Goldman HM, Cohen DW, Eds. Contemporary periodontics. C.V. Mosby Company. St. Louis, pp 184-93. 
Gibbons RJ, Socransky SS (1966). Enhancement of alveolar bone loss in gnotobiotic mice harbouring human gingival bacteria. Arch Oral Biol 11: 847-8.

Giddon DB, Clark E, Varni JG (1969). Apparent digital vasomotor hypotonicity in the remission stage of acute necrotizing ulcerative gingivitis. J Dent Res 48: 431-8.

Giddon DB, Goldhaber P, Dunning JM (1963). Prevalence of reported cases of acute necrotising ulcerative gingivitis in a university population. $J$ Periodontol 34: 366-71.

Giddon DB, Zackin SJ, Goldhaber P (1964). Acute necrotizing ulcerative gingivitis in college students. J Am Dent Assoc 68: 381-6.

Giovanni M, Zuccoti GV, Fiocchi A (1989). Gangrenous Stomatitis in a child with AIDS (Letter). Lancet 2: 1400.

Glick M, Cleveland DB, Salkin LM, Alfaro-Miranda M, Fielding AF (1991). Intraoral cytomegalovirus lesion and HIV-associated periodontitis in a patient with acquired immunodeficiency syndrome. Oral Surg Oral Med Oral Pathol 72: 716-20.

Glick M, Muzyka BC, Salkin LM, Lurie D (1994). Necrotizing ulcerative periodontitis: a marker for immune deterioration and a predictor for the diagnosis of AIDS. $J$ Periodontol 65: 393-97.

Glick M, Pliskin ME, Weiss RC (1990). The clinical and histologic appearance of HIVassociated gingivitis. Oral Surg Oral Med Oral Pathol 69: 395-8.

Goldhaber P, Giddon DB (1964). Present concepts concerning the etiology and treatment of acute necrotizing ulcerative gingivitis. Int Dent J 14: 468-96.

Gomez RS, Costa JE, Loyola AM, Araújo NS, Araújo VC (1995). Immunohistochemical study of linear gingival erythema from HIV-positive patients. $J$ Periodont Res 30: 355-59. Gornitsky M, Pekovic D (1987). Involvement of human immunodeficiency virus (HIV) in gingiva of patients with AIDS. Adv Exp Med Biol 21: 553-62.

Grant DA, Stern IB, Listgarten MA (1988). Periodontics In the tradition of Gottlieb and Orban. The C.V. Mosby Company, St. Louis.

Grassi M, Hammerle C, Staschek I, Berthold H, Lang NP (1990). Longitudinal observation of HIV-associated gingivitis. 6th International Conference on AIDS, San Francisco. 
Grassi M, Williams CA, Winkler JR, Murray PA (1988). Management of HIV-associated periodontal diseases. In: Robertson PB, Greenspan JS, Eds. Perspectives on Oral Manifestations of AIDS. PSG Publishing Company, inc. Littleton, pp 119-30.

Grbic JT, Mitchell-Lewis DA, Fine JB, Phelan JA, Bucklan RB, Zambon JJ, Lamster IB (1995). The relationship of candidiasis to Linear Gingival Erythema in HIV-infected homosexual men and parenteral drug users. J Periodontol 66: 30-7.

Green LW, Tryon WW, Marks B, Huryn J (1986). Psychosomatic factors in periodontal disease. $J$ Human Stress 12: 32-6.

Greene JC (1990). General principles of epidemiology and methods for measuring prevalence and severity of periodontal disease. In: Genco RJ, Goldman HM, Cohen DW, Eds. Contemporary Periodontics. The C.V. Mosby Company. St. Louis, pp 97-105.

Greene JC, Vermillion JR (1960). The oral hygiene index: a method for classifying oral hygiene status. J Am Dent Assoc 61: 172-9.

Greene JC, Vermillion JR (1964). The Simplified Oral Hygiene Index. J Am Dent Assoc 68: 7-13.

Greenspan D, Greenspan JS (1992). Significance of oral hairy leukoplakia. Oral Surg Oral Med Oral Pathol 73: 151-4.

Greenspan D, Greenspan JS, Pindborg JJ, Schiodt M (1986). AIDS and the dental team. Munksgaard, Copenhagen.

Greenspan D, Greenspan JS, Schiodt M, Pindborg JJ (1990). AIDS and the mouth. Munksgaard, Copenhagen.

Greenspan JS, Barr CE, Sciubba JJ, Winkler JR and the US Oral AIDS Collaborative Group (1992). Oral manifestations of HIV infection. Definitions, diagnostic criteria, and principles of therapy. Oral Surg Oral Med Oral Pathol 73: 142-44.

Grieve WG, Winkler JR, Murray PA (1988). HIV sero-positive individuals humoral immune response to periodontal pathogens in HIV-P. 4th International Conference on AIDS, Stockholm. 
Griffiths GS, Wilton JMA, Curtis MA, Maiden MFJ, Gillett IR, Wilson DT, Sterne JAC, Johnson NW (1988). Detection of high-risk groups and individuals for periodontal diseases. Clinical assessment of the periodontium. J Clin Periodontol 15: 403-10.

Gunsolley JC, Best AM (1988). Change in attachment level. J Periodontol 59: 450-6.

Gupta OP, Blechman H, Stahl SS (1960). Effects of stress on the periodontal tissues of young male rats and hamsters. $J$ Periodontol 32: 413-7.

Haber J, Wattles J, Crowley M, Mandell R, Joshipura K, Kent RL (1993). Evidence of cigarette smoking as a major risk factor for periodontitis. J Periodontol 64: 16-23.

Haffajee AD, Socransky SS, Goodson JM, Lindhe J (1985). Intraclass correlations of periodontal measurements. J Clin Periodontol 12: 216-24.

Haffajee AD, Socransky SS, Lindhe J (1983). Comparison of statistical methods of analysis of data from clinical periodontal trials. J Clin Periodontol 10: 247-56.

Hampp EG, Mergenhagen SE (1961). Experimental infections with oral spirochetes. $J$ Infect Dis 109: 43-61.

Hart TC, Shapira L, Van Dyke TE (1994). Neutrophil defects as risk factors for periodontal diseases. J Periodontol 65: 521-29.

Hassell TM (1990). Local and systemic actions of drugs and other chemical agents on periodontal tissues. In: Genco RJ, Goldman HM, Cohen DW, Eds. Contemporary periodontics. C.V. Mosby Company. St. Louis, pp 269-76.

Henshaw NE, Adenjubi JO (1975). Periodontal disease in northern zone of Nigeria. Nig Med J 5: 152-9.

Hernichel-Gorbach E, Kornman KS, Holt SC, Nicholls F, Meador H, Kung JT, Thomas CA (1994). Host responses in patients with generalised refractory periodontitis. $J$ Periodontol 65: 8-16.

Holt SC, Ebersole J, Felton J, Brunsvold M, Kornman KS (1988). Implantation of Bacteroides gingivalis in non human primates initiates progression of periodontitis. Science 239: 55-7. 
Imrey PB (1986). Considerations in the statistical analysis of clinical trials in periodontitis. $J$ Clin Periodontol 13: 517-28.

Ismail AI, Morrison EC, Burt BA, Caffesse RG, Kavanagh MT (1990). Natural history of periodontal disease in adults: findings from the Tecumseh periodontal disease study, 195987. J Dent Res 69(2): 430-5.

Israel DS, Plaisance KI (1991). Neutropenia in patients infected with human immunodeficiency virus. Clin Pharm 10: 268-279.

Janssen PTM, Faber JAJ, van Palenstein Helderman WH (1988). Effect of probing depth and bleeding tendency on the reproducibility of probing depth measurements. $J$ Clin Periodontol 15: 565-8.

Jasani MK (1979). Anti-inflammatory steroids: Mode of action in rheumatoid arthritis and homograft rejection. In: Vane JR, Ferreira SH, Eds. Anti-inflammatory drugs. SpringerVerlag. Berlin, pp 588-660.

Johannessen AC, Nilsen R, Knudsen GE, Kristoffersen T (1986). In situ characterization of mononuclear cells in human chronic marginal periodontitis using monoclonal antibodies. $J$ Periodont Res 21: 113-27.

Johnson BD, Engel D (1986). Acute necrotising ulcerative gingivitis. A review of diagnosis, etiology and treatment. J Periodontol 57: 141-50.

Johnson NW (1991). Introduction: current concepts of the nature and the natural history of the periodontal diseases and the need for disease markers. In: Johnson NW, Ed. Risk markers for oral diseases. Volume 3: Periodontal diseases: markers of disease activity and susceptibility. Cambridge University Press. Cambridge, pp 1-5.

Kaposi M (1872). Idiopathisches multiples pigmentsarkom der haut. Arch Dermatol Syph 4: 265-73.

Kardachi JR, Newcomb GM (1978). A clinical study of gingival inflammation in renal transplant recipients taking immunosuppressive drugs. J Periodontol 49: 307-9.

Kass E (1970). Bacteria in periodontal diseases. J Dent Res 49: 229-32. 
Katz MH, Greenspan D, Westenhouse J, Hessol NA, Buchbinder SP, Lifson AR, Shiboski S, Osmond D, Moss A, Samuel M, Lang W, Feigal DW, Greenspan JS (1992). Progression to AIDS in HIV-infected homosexual and bisexual men with hairy leukoplakia and oral candidiasis. AIDS 6: 95-100.

Khoo KK, Watts TLP (1988). Upper anterior tooth mobility. J Periodontol 59: 231-7.

Kinane DF, Johnston : FA, Evans CW (1989). Depressed helper-to-suppressor T-cell ratios in early-onset forms of periodontal disease. J Periodont Res 24: 161-4.

King SH (1972). Social-psychological factors in illness. In: Freeman HELS, Reeder LG, Eds. Handbook of Medical Sociology. International Universities Press, Inc. Madison, pp 129-137.

Kingman A, Löe H, Anerud A, Boysen H (1991). Errors in measuring parameters associated with periodontal health and disease. J Periodontol 62: 477-86.

Klein RS, Quart AM, Butkus Small C (1991). Periodontal disease in heterosexuals with acquired immunodeficiency syndrome. J Periodontol. 62: 535-40.

Korting HC, Ollert M, Georgii A, Froschl M (1989). In vitro susceptibilities and biotypes of Candida albicans isolates from the oral cavities of patients infected with human immunodeficiency virus. J Clin Microbiol 26: 2626-31.

Kranzler HR, Babor TF, Goldstein L, Gold J (1990). Dental pathology and alcohol related indicators in an outpatient sample. Community Dent Oral Epidemiol 18: 204-7.

Kucers A, Bennet NM (1987). The use of antibiotics. William Heinemann, London.

Lamster I, Grbic J, Fine J, Bucklan R, Mitchell-Lewis D, Phelan J, Zambon J (1995). A critical review of periodontal disease as a manifestation of HIV infection. In: Greenspan JS, Greenspan D, Eds. Oral Manifestations of HIV Infection. Quintessence Publishing Co, Inc. Chicago, pp 247-56.

Lamster IB, Oshrain RL, Harper DS (1987). Infantile agranulocytosis with survival into adolescence. Periodontal manifestations and laboratory findings. J Periodontol 58: 34-9. 
Lang NP (1991). Clinical markers of active periodontal disease. In: Johnson NW, Ed. Risk markers of oral diseases. Volume 3: Periodontal diseases: Markers of disease susceptibility and activity. Cambridge University Press. Cambridge, pp 179-202.

Lang W, Perkins H, Anderson RE, Royce R, Jewell N, Winkelstein WJ (1989). Patterns of $T$ lymphocyte changes with Human Immunodeficiency Virus infection: From seroconversion to the development of AIDS. J AIDS 2: 63-9.

Langford AA, Reichart P, Pohle HD (1988). Oral manifestations associated with HIVinfection. (Abstract). 4th International conference on AIDS, Stockholm.

Laskaris G, Potouridou I, Laskaris M, Stratigos J (1992). Gingival lesions of HIV infection in 178 Greek patients. Oral Surg Oral Med Oral Pathol 74: 168-71.

Lazzarin A, Uberti Foppa C, Galli M, Mantovani A, Poli G, Franzetti F, Novati R (1986). Impairment of polymorphonuclear leucocyte function in patients with acquired immunodeficiency syndrome and with lymphadenopathy syndrome. Clin Exp Immunol 65: 105-11.

Lemp GF, Hirozawa AM, Givertz D, Nieri GN, Anderson L, Lindegren ML, Janssen RS, Katz M (1994). Seroprevalence of HIV and risk behaviours among young homosexual and bisexual men. The San Francisco/Berkeley young men's survey. J Am Med Assoc. 272: 44954.

Levine RA, Glick M (1991). Rapidly progressive periodontitis as an important clinical marker for HIV disease. Compendium 12: 478-88.

Lifson AR (1992). Transmission of the human immunodeficiency virus. In: DeVita VT, Hellman S, Rosenberg SA, Eds. AIDS. Etiology, diagnosis, treatment and prevention. J.B. Lippincott Company. Philadelphia, pp 111-20.

Lifson AR, Hilton JF, Westenhouse JL, Canchola AJ, Samuel MC, Katz MH, Buchbinder SP, Hessol NA, Osmond DH, Shiboski S (1994). Time from seroconversion to oral candidiasis or hairy leukoplakia among homosexual and bisexual men enrolled in three prospective cohorts. AIDS 8: 73-9.

Listgarten MA (1965). Electron microscopic observations on the bacterial flora of acute necrotising ulcerative gingivitis. J Periodontol 36: 328-39. 
Löe H, Anerud A, Boysen H, Morrison E (1986). Natural history of periodontal disease in man. Rapid, moderate and no loss of attachment in Sri Lankan laborers 14 to 46 years of age. J Clin Periodontol 13: 431-45.

Löe H, Anerud A, Boysen H, Smith M (1978). The natural history of periodontal disease in man. J Periodontol 49: 607-20.

Löe H, Morrison E (1990). Epidemiology of periodontal disease. In: Genco RJ, Goldman HM, Cohen DW, Eds. Contemporary periodontics. C.V. Mosby. St. Louis, pp 106-16.

Löe H, Schiott CR (1970). The effect of mouthrinses and the topical application of chlorhexidine on the development of dental plaque and gingivitis in man. J Periodont Res 5: 79-83.

Löe H, Silness J (1963). Periodontal disease in pregnancy. I. Prevalence and severity. Acta Odontol Scand 21: 533-51.

Löe H, Theilade E, Jensen SB (1965). Experimental gingivitis in man. J Periodontol 36: 177-87.

Loesche WJ, Syed SA, Laughon BE, Stoll J (1982). The bacteriology of acute necrotising ulcerative gingivitis. J Periodontol 53: 223-30.

Lovdal A, Arno A, Waerhaug J (1958). Incidence of clinical manifestations of periodontal disease in light of oral hygiene and calculus formation. J Am Dent Assoc 56: 21-33.

Lozada F, Silverman SJ, Conant M (1982). New outbreak of oral tumors, malignancies and infectious diseases that strikes young male homosexuals. J Calif Dent Assoc 10: 39-42.

Lucht E, Heimdahl A, Nord CE (1991). Periodontal disease in HIV-infected patients in relation to lymphocyte subsets and specific micro-organisms. J Clin Periodontol 18: 252-6.

Lucht E, Teivens A, Berglund O, Heimdahl A, Nord CE (1990). Occurrence of microorganisms in saliva and gingival crevice in HIV-1-infected patients. Microbial Ecol Health Dis 3: 329-34.

Lynch DP (1994). Oral Candidiasis. History, classification, and clinical presentation. Oral Surg Oral Med Oral Pathol 78: 189-93. 
MacDonald JB, Gibbons RJ, Socransky SS (1960). Bacterial mechanisms in periodontal disease. Ann NY Acad Sci 85: 467-78.

MacDonald JB, Sutton RM, Knoll ML, Madlener EM, Grainger RM (1956). The pathogenic components of an experimental fusospirochetal infection. J Infect Dis 98: 15-20.

MacFarlane GD, Herzberg MC, Wolff LM, Hardie NA (1992). Refractory periodontitis associated with abnormal polymorphonuclear leukocyte phagocytosis and cigarette smoking. J Periodontol 63: 908-13.

Magnusson I, Fuller WW, Heins PJ, Rau CF, Gibbs CH, Marks RG, Clark WB (1988). Correlation between electronic and visual readings of pocket depths with a newly developed constant force probe. J Clin Periodontol 15: 180-4.

Malberg K, Molle A, Streuer D, Gangler P (1992). Determination of lymphocyte populations and subpopulations extracted from chronically inflamed human periodontal tissues. J Clin Periodontol 19: 155-8.

Mandel ID (1974). Indices for measurement of soft accumulations in clinical studies of oral hygiene and periodontal disease. J Periodont Res 9: 7-30.

Manhold JH, Doyle JL, Weisinger EH (1971). Effects of social stress on oral and other bodily tissues. II. Results offering substance to a hypothesis for the mechanism of periodontal pathology. J Periodontol 42: 109-11.

Mann JM, Chin J, Piot P, Quinn T (1988). The international epidemiology of AIDS. Sci Am 259: 82-9.

Manson JD, Rand H (1961). Recurrent Vincent's disease. A survey of 61 cases. Br Dent J 110: $386-90$.

Markitziu A, Zafiropoulos G, Flores de Jacoby L, Pisanty S (1990). Periodontal alterations in patients with pemphigus vulgaris taking steroids. J Clin Periodontol 17: 228-32.

Martínez-Canut P, Guarinos J, Bagan JV (1996). Periodontal disease in HIV seropositive patients and its relation to lymphocyte subsets. $J$ Periodontol 67: 33-6.

Martínez-Canut P, Lorca A, Magan R (1995). Smoking and periodontal disease severity. $J$ Clin Periodontol 22: 743-9. 
Masouredis CM, Katz MH, Greenspan D, Herrera C, Hollander H, Greenspan JS, Winkler JR (1992). Prevalence of HIV-associated periodontitis and gingivitis in HIV-infected patients attending an AIDS clinic. J AIDS 5: 479-83.

Maupin CC, Bell WB (1975). The relationship of 17-Hydroxycorticosteroid to acute necrotizing ulcerative gingivitis. J Periodontol 46: 721-2.

McCance-Katz EF, Hoecker JL, Vitale NB (1987). Severe neutropenia associated with antineutrophil antibody in a patient with acquired immunodeficiency syndrome-related complex. Pediatr Infect Dis 6: 417-8.

Melnick SL, Engel D, Truelove E, DeRouen T, Morton T, Schubert M, Dunphy C, Wood RW (1989). Oral mucosal lesions: Association with the presence of antibodies to the human-immunodeficiency virus. Oral Surg Oral Med Oral Pathol 68: 37-43.

Meng H, Zheng L (1990). T cells and T-cell subsets in periodontal diseases. J Periodontol 61: 121-6.

Mertens TE (1993). Estimating the effects of misclassification. Lancet 342: 418-21.

Mikx FHM, van Campen GJ (1982). Microscopical evaluation of the microflora in relation to necrotising ulcerative gingivitis in the beagle dog. J Periodont Res 17: 576-84.

Miller AJ, Brunelle JA, Carlos JP, Brown LJ, Löe H (1987). Oral health of United States adults; The national survey of oral health in U.S. employed adults and seniors: 1985-6. NIH.

Miller SC, Thaller JL, Soberman A (1956). The use of the Minnesota multiphasic personality inventory as a diagnostic aid in periodontal disease - a preliminary report. $J$ Periodontol 27: 44-6.

Moore LVH, Moore WEC, Riley C, Brooks CN, Burmeister JA, Smibert RM (1993). Periodontal microflora of HIV positive subjects with gingivitis or adult periodontitis. $J$ Periodontol 64: 48-56.

Moskow BS, Hernandez G (1991). Aggressive periodontal destruction and herpes zoster in a suspected AIDS patient. J Parodontol 10: 359-69.

Moulton R, Ewen S, Thieman W (1952). Emotional factors in periodontal disease. Oral Surg 5: 833-60. 
Muhlemann HR, Mazor ZS (1958). Gingivitis in Zurich school children. Helv Odontol Acta 2: 3-10.

Mullaly BH, Linden GJ (1994). Comparative reproducibility of proximal probing depth using electronic pressure-controlled and hand probing. J Clin Periodontol 21: 284-8.

Murray P, Fenesy K, Scorziello T, Lynch M, Najjar T, Jandinski J (1991). HIV-associated periodontal disease in IV-drug users (Abstract). J Dent Res 70: 415.

Murray PA, Grassi M, Winkler JR (1989). The microbiology of HIV-associated periodontal lesions. J Clin Periodontol 16: 636-42.

Murray PA, Grieve WG, Winkler JR (1987). The humoral immune response in HIVassociated periodontitis (Abstract). 3rd International Conference on AIDS, Washington,

Murray PA, Winkler JR, Peros WJ, French CK, Lippke JA (1991). DNA probe detection of periodontal pathogens in HIV-associated periodontal lesions. Oral Microbiol Immunol 6: 34-40.

Murray PA, Winkler JR, Sadowski L, Kornman KS, Steffensen B, Robertson PB, Holt SC (1988). Microbiology of HIV-associated gingivitis and periodontitis. In: Robertson PB, Greenspan JS, Eds. Perspectives on Oral Manifestations of AIDS. PSG Publishing Company, inc. Littleton, pp 105-18.

Nadeau D, Boyer R, Fortin C, Duval B, Godin G, Bergeron M (1993). Facteurs associes aux relations anales a risque chez des hommes homosexuels seropositifs au VIH. Revue d'Epidemiologie et de Sante Publique 41: 228-34.

Newman TB, Browner WS, Cummings SR, Hulley SB (1988). Designing a new study: II. Cross-sectional and case-control studies. In: Hulley SB, Cummings SR, Eds. Designing clinical research. Williams \& Wilkins. Baltimore, pp 75-86.

Newman TB, Browner WS, Hulley SB (1988). Enhancing causal inference in observational studies. In: Hulley BB, Cummings SR, Eds. Designing clinical research. Williams and Wilkins. Baltimore, pp 98-109.

Odden K, Schenck K, Hurlen B (1995). High numbers of T cells in gingiva from patients with human immunodeficiency virus (HIV) infection. J Oral Pathol Med 24: 413-9. 
Odden K, Schenck K, Koppang HS, Hurlen B (1994). Candidal infection in the gingiva in HIV-infected persons. J Oral Pathol Med 23: 178-83.

Odds FC (1988). Candida and Candidosis. Bailliere Tindall, London.

Okada H, Kida T, Yamagami H (1983). Identification and distribution of immunocompetent cells in inflamed gingiva of human chronic periodontitis. Infec Immun 41: 365-374.

OPCS (1991). Standard occupational classification. 1990. HMSO, London.

Oshrain HI, Mender S, Mandel ID (1979). Periodontal status of patients with reduced immunocapacity. J Periodontol 50: 185-88.

Oshrain HI, Telsey B, Mandel ID (1983). Longitudinal study of periodontal disease in patients with reduced immunocapacity. J Periodontol 54: 151-4.

Osuji OO (1990). Necrotising ulcerative gingivitis and cancrum oris (noma) in Ibadan, Nigeria. J Periodontol 61: 769-72.

Page RC, Altman LC, Ebersole JL, Vandesteen GE, Dahlberg WH, Williams BL, Osterberg SK (1983). Rapidly Progressive Periodontitis. A distinct clinical condition. J Periodontol 54: 197-209.

Palmer GD, Morgan PR, Challacombe SJ (1993). T-cell lymphoma associated with periodontal disease and HIV infection. A case report. J Clin Periodontol 20: 378-80.

Palmer GD, Robinson PG, Challacombe SJ, Birnbaum W, Croser D, Erridge PL, Hodgson T, Lewis D, McLaren A, Zakrzewska JM (1996). Aetiological factors for oral manifestations of HIV infection. Submitted, Oral Diseases.

Palmer RM (1987). Tobacco smoking and oral health. Health Education Authority, London.

Papapanou PN, Lindhe J (1992). Preservation of probing attachment and alveolar bone levels in 2 random population samples. J Clin Periodontol 19: 583-88.

Pekovic DD, Lawton R, Adamkiewicz VW, Gornitsky M, Ajdukovic D (1988). AIDS associated periodontal disease (Abstract). 4th International Conference on AIDS, Stockholm, 
Pettiti DB, Friedman GB, Kahn W (1981). Accuracy of information on smoking habits provided on self-administered research questionnaires. Am J Public Health 71: 308-11.

Phelan JA, Saltzmann BR, Friedland GH, Klein RS (1987). Oral findings in patients with acquired immunodeficiency syndrome. Oral Surg Oral Med Oral Pathol 64: 50-6.

Pierce JP, Dwyer T, Digiusto E, Carpenter T, Hannam C, Amin A, Yong C, Sarfaty K, Shaw J, Burke N (1987). Cotinine validation of self-reported smoking in commercially run community surveys. J Chronic Diseases 40: 689-95.

Pihlstrom BL (1992). Measurement of attachment level in clinical trials: probing methods. $J$ Periodontol 63: 1072-7.

Pindborg JJ (1951). Influence of service in armed forces on incidence of gingivitis. $J$ Am Dent Assoc 42: 517-22.

Pindborg JJ (1989). Classification of Oral Lesions associated with HIV infection. Oral Surg Oral Med Oral Pathol 67: 292-95.

Pindborg JJ, Holmstrup P (1987). Necrotizing gingivitis related to human immunodeficiency virus (HIV) infection. Afr Dent $J$ 1: 5-8.

Pindborg JJ, Thorn JJ, Schiodt M, Gaul J, Black FT (1986). Acute Necrotizing Ulcerative Gingivitis in a Danish AIDS patient. Tandlaegebladet 90: 450-3.

Pollmann L, Dietrich A (1979). Acute necrotising ulcerative gingivitis in young men. Deutsche Zahnartzliche Zeitschrift 34: 222-4.

Porter S, Scully C (1994). HIV: the surgeon's perspective. Part 3. Diagnosis and management of malignant neoplasms. Br J Oral Maxillofac Surg 32: 241-47.

Porter SR, Scully C (1993). Orofacial manifestations in primary immunodeficiencies: polymorphonuclear leukocyte defects. J Oral Pathol Med 22: 310-1.

Porter SR, Scully C (1993). Orofacial manifestations in primary immunodeficiencies: T lymphocyte defects. J Oral Pathol Med 22: 308-9.

Preber H, Bergstrom J (1985). The effect of non-surgical treatment on periodontal pockets in smokers and non-smokers. J Clin Periodontol 13: 319-23. 
Preber H, Bergstrom J (1990). Effect of cigarette smoking on periodontal healing following surgical therapy. J Clin Periodontol 17: 324-8.

Preber H, Bergstrom J, Linder LE (1992). Occurrence of periopathogens in smoker and non-smoker patients. J Clin Periodontol 19: 667-71.

Quigley G, Hein J (1962). Comparative cleansing efficiency of manual and power brushing. J Am Dent Assoc 65: 26-9.

Ralls SA, Cohen ME (1986). Problems in identifying 'bursts' of periodontal attachment loss. J Periodontol 57: 746-52.

Ramfjord SP (1967). The periodontal disease index (PDI). J Periodontol 38: 30-8.

Rams TE, Andriolo MJ, Feik D, Abel SN, McGivern TM, Slots J (1991). Microbiological study of HIV-related periodontitis. J Periodontol 62: 74-81.

Ras GJ, Eftychis HA, Anderson R, Van der Walt I (1984). Mononuclear and polymorphonuclear leucocyte dysfunction in male homosexuals with the acquired immunodeficiency syndrome (AIDS). S Afr Med J 66: 806-9.

Ratcliff PA (1956). The relationship of the general adaptive response to the periodontal tissues in the rat. $J$ Periodontol 27: 40-3.

Reinhardt RA, Bolton RW, McDonald TL, DuBois LM, Kaldahl WB (1988). In situ lymphocyte populations from active versus stable periodontal lesions. J Periodontol 59: 656-70.

Riley C, London JP, Burmeister JA (1992). Periodontal health in 200 HIV-positive patients. J Oral Pathol Med 21: 124-7.

Robinson PG (1992). Periodontal diseases and HIV infection. J Clin Periodontol 19: 60914.

Robinson P, Farthing P, Scott GM, Bennett JH (1996) Oral Mycobacterium avium complex infection in a man with AIDS. Oral Surg Oral Med Oral Pathol 81: 177-9.

Robinson PG, Winkler JR, Palmer G, Westenhouse J, Hilton JF, Greenspan JS (1994). The diagnosis of periodontal conditions associated with HIV infection. $J$ Periodontol 65:236-43. 
Robinson PG, Zakrzewska JM (1994). Gingival ulceration associated with HIV infection (Abstract). J Dent Res 64: 827.

Roilides E, Holmes A, Blake C, Pizzo PA, Walsh TJ (1993). Impairment of neutrophil antifungal activity against hyphae of Aspergillus fumigatus in children infected with human immunodeficiency virus. J Infect Dis 167: 905-11.

Rosebury T, MacDonald JB, Clark AR (1950). A bacteriologic survey of gingival scrapings from periodontal infections by direct examination, guinea pig inoculation and anaerobic cultivation. J Dent Res 29: 718-31.

Rosenberg ZF, Fauci AS (1992). Immunopathogenesis of HIV infection. In: DeVita VT, Hellman S, Rosenberg SA, Eds. AIDS. Etiology, diagnosis, treatment and prevention. J.B. Lippincott Company. Philadelphia, pp 61-76.

Rosenstein DI, Eigner TL, Levin MP, Chiodo GT (1989). Rapidly progressive periodontal disease associated with HIV infection: report of a case. J Am Dent Assoc. 118: 313-4.

Rosenstein DI, Riviere GR, Elott KS (1993). HIV-associated periodontal disease: new oral spirochaete found. J Am Dent Assoc 124: 76-80.

Roth H (1951). Psychosomatic and nutritional factors related to recurrent necrotizing ulcerative gingivitis. J Am Dent Assoc 42: 474-5.

Rothman KJ (1986). Modern Epidemiology. Little, Brown and Company, Boston.

Ryder MI, Winkler JR, Weinreb RN (1988). Elevated phagocytosis, oxidative burst, and Factin formation in PMNs from individuals with intra-oral manifestations of HIV infection. $J$ AIDS 1: 346-53.

Saadoun AP (1987). Les maladies parodontales et la Sida. J Parodontol 6: 345-55.

Safkan B, Knuuttila M (1984). Corticosteroid therapy and periodontal disease. J Clin Periodontol 11: 515-22.

Sakki TK, Knuuttila MLE, Vimpari SS, Hartikainen MSL (1995). Association of lifestyle with periodontal health. Community Dent Oral Epidemiol 23: 155-8. 
San Martin T, Jandinski JJ, Palumbo P, Murray PA (1992). Periodontal diseases in children infected with HIV (Abstract). J Dent Res 71: 151.

SanGiancomo TR, Tan PM, Loggi DG, Itkin AB (1990). Progressive osseous destruction as a complication of HIV-Periodontitis. Oral Surg Oral Med Oral Pathol 70: 476-479.

Saxer UP, Muhlemann HR (1975). Motwation und aufklarung. Monatsscher Zahnheilk 85: 905-19.

Schiodt M (1992). HIV-associated salivary gland disease: A review. Oral Surg Oral Med Oral Pathol 73: 164-7.

Schiodt M, Bakilana PB, Hiza JFR, Shao JF, Bygbjerg IB, Mbaga I, Vestergaard BF, Nielsen CM, Lauritzen E, Lerche B, Kuijlen K (1990). Oral candidiasis and hairy leukoplakia correlate with HIV infection in Tanzania. Oral Surg Oral Med Oral Pathol 69: 591-6.

Schlesselman JJ (1982). Case-control studies. Design, conduct, analysis. Oxford University Press, Oxford.

Schluger S (1943). The etiology and treatment of Vincent's infection. J Am Dent Assoc 29: 524-32.

Schour I, Massler M (1958). Prevalence of gingivitis in young adults (Abstract). J Dent Res 27: 733-4.

Schuller PD, Freedman HL, Lewis DW (1973). Periodontal status of renal transplant patients receiving immunosuppressive therapy. J Periodontol 44: 167-70.

Schulten EAJM, Ten Kate RW, Van Der Waal I (1990). Oral findings in HIV-infected patients attending a department of internal medicine: The contribution of intraoral examination towards the clinical management of HIV disease. $Q J$ Med 76: 741-5.

Scully C, Epstein JB, Porter S, Luker J (1990). Recognition of oral lesions of HIV infection. 3. Gingival and periodontal disease and less common lesions. Br Dent J 169: 3702.

Seymour GJ (1987). Possible mechanisms involved in the immunoregulation of chronic inflammatory periodontal disease. J Dent Res 66: 2-9. 
Seymour RA, Smith DG, Rogers SR (1987). The comparative effects of azathioprine and cyclosporin on some gingival health parameters of renal transplant patients. A longitudinal study. J Clin Periodontol 14: 610-3.

Sheiham A (1966). An epidemiological survey of acute ulcerative gingivitis in Nigerians. Archs Oral Biol 11: 937-44.

Shiboski CH, Winkler JR (1993). Gingival Kaposi's sarcoma and periodontitis. Oral Surg Oral Med Oral Pathol 76: 49-53.

Shklar G (1966). Periodontal disease in experimental animals subjected to chronic cold stress. J Periodontol 37: 377-82.

Silness J, Löe H (1964). Periodontal disease in pregnancy. II. Correlation between oral hygiene and periodontal condition. Acta Odontol Scand 22: 121-35.

Silverman SJ, Migliorati CA, Lozada-Nur F, Greenspan D, Conant M (1986). Oral findings in people with or at risk for AIDS: a study of 375 homosexual males. J Am Dent Assoc 112: 187-92.

Silverstein SJ (1973). Relation between social drug use/abuse and dental disease in California, USA. Community Dent Oral Epidemiol 1: 89-93.

Sitthi-amorn C, Poshyachinda V (1993). Bias. Lancet 342: 286-8.

Slots J, Genco RJ (1984). Black-pigmented Bacteroides species, Capnocytophaga species, and Actinobacillus actinomycetemcomitans in human periodontal disease: virulence factors in colonization, survival, and tissue destruction. J Dent Res 63: 412-21.

Slots J, Rams TE (1990). Antibiotics in periodontal therapy. Advantages and disadvantages. J Clin Periodontol. 17: 479-93.

Slots J, Rams TE, Listgarten MA (1988). Yeasts, enteric rods, and pseudomonads in the subgingival flora of severe adult periodontitis. Oral Microbiol Immunol 3: 47-52.

Smith GLF, Cross DL, Wray D (1995). Comparison of periodontal disease in HIV seropositive subjects and controls (1). Clinical features. J Clin Periodontol 22: 558-68. 
Smith GLF, Felix DH, Wray D (1991). HIV associated periodontal disease: prevalence, clinical features and management (Abstract). J Dent Res 70: 688.

Smith GLF, Felix DH, Wray D (1993). Current classifications of HIV-associated periodontal diseases. Br Dent J 174: 102-5.

Smith LW, Suomi JD, Greene JC, Barbano JP (1970). A study of intra-examiner variation in scoring oral hygiene status, gingival inflammation and epithelial attachment level. $J$ Periodontol 41: 671-74.

Socransky SS, Haffajee AD, Goodson JM, Lindhe J (1984). New concepts of destructive periodontal disease. J Clin Periodontol 11: 21-32.

Sohoel PDC, Johannessen AC, Kristoffersen T, Haugstvedt Y, Nilson R (1992). In situ characterisation of mononuclear cells in marginal periodontitis of patients with Down's syndrome. Acta Odontol Scan 50: 141-9.

Soubry R, Taelman H, Banyangiliki V, Ladner J, Van de Pere P (1995). Necrotising periodontal disease in HIV-1 infected patients: a 4 year study. In: Greenspan JS, Greenspan D, Eds. Oral Manifestations of HIV Infection. Quintessence Publishing Co, Inc. Chicago, pp 60-7.

Stahl SS (1961). Healing gingival injury in normal and systemically stressed young adult male rats. $J$ Periodontol 32: 63-73.

Stammers AF (1944). Vincent's infection observations and conclusions regarding the aetiology and treatment of 1,017 civilian cases. Br Dent J 76: 147-55.

Steidley KE, Thompson SH, McQuade MJ, Strong SL, Scheidt MJ, Van Dyke TE (1992). A comparison of T4:T8 lymphocyte ratio in the periodontal lesion of healthy and HIVpositive patients. $J$ Periodontol 63: 753-6.

Sterne JAC, Curtis MA, Gillett IR, Griffiths GS, Maiden MFJ, Wilton JMA, Johnson NW (1990). Statistical models for data from periodontal research. J Clin Periodontol 17: 129-37.

Stoltenberg JL, Osborn JB, Pihlstrom BL, Herzberg MC, Aeppli DM, Wolff LF, Fischer GE (1993). Association between cigarette smoking, bacterial pathogens, and periodontal status. J Periodontol 64: 1225-30. 
Suomi JD, Barbano JP (1968). Patterns of gingivitis. J Periodontol 39: 71-4.

Suomi JD, Greene JC, Vermillion JR, Doyle J, Chang JJ, Leatherwood EC (1971). The effect of controlled oral hygiene procedures on the progression of periodontal disease in adults. J Periodontol 42: 152-60.

Sutton RBO, Smales FC (1983). Cross-sectional study of the effects of immunosuppressive drugs on chronic periodontal disease in man. J Clin Periodontol 10: 317-26.

Swango PA, Kleinman DV, Konzelman JL (1991). HIV and periodontal health. A study of military personnel with HIV .J Am Dent Assoc 122: 49-54.

Syrjänen S, Markkanen H, Syrjänen K (1984). Inflammatory cells and their subsets in lesions of juvenile periodontitis. Acta Odontol Scand 42: 285-92.

Tan EM, Cohen AS, Fries JF, Masi AT, McShane DJ, Rothfield NF, Schaller JG, Talal N, Winchester RJ (1982). The 1982 revised criteria for the classification of systemic lupus erythematosus. Arthritis Rheum 25: 1271-77.

Taubman MA, Stoufi ED, Ebersole JL, Smith DJ (1984). Phenotypic studies of cells from periodontal disease tissues. J Periodont Res 19: 587-90.

Tenenbaum HC, Mock D, Simor AE (1991). Periodontitis as an early presentation of HIV infection. J Can Med Assoc 144: 1265-9.

Theil EM, Heaney TG (1991). The validity of periodontal probing as a method of measuring loss of attachment. J Clin Periodontol 18: 648-53.

Thompson SH, Charles GA, Craig DB (1992). Correlation of oral disease with Walter Reed staging scheme for HIV-1 seropositive patients. Oral Surg Oral Med Oral Pathol 73: 28992.

Todd JE, Lader D (1991). Adult Dental Health 1988 United Kingdom. OPCS, London.

Tollefsen T, Johansen JR (1985). The periodontal status of prospective and renal transplant patients. J Periodont Res 20: 220-6. 
Tukutuku K, Muyembe-Tamfum L, Kayembe K, Mavuemba T, Sangua N, Sekele I (1990). Prevalence of dental caries, gingivitis, and oral hygiene in hospitalized AIDS cases in Kinshasa, Zaire. J Oral Pathol Med 19: 271-2.

Turesky S, Gilmore N, Glickman I (1970). Reduced plaque formation by the chloromethyl analogue of vitamin C. J Periodontol 41: 41-3.

Vallejo GH, Garcia MD, Lopez A, Mendieta C, Moskow BS (1989). Unusual periodontal findings in an AIDS patient with Burkitt's lymphoma. J Periodontol 60: 723-7.

Watts TLP (1987). Constant force probing with and without a stent in untreated periodontal disease: the clinical reproducibility and possible sources of error. $J$ Clin Periodontol 14: 407-411.

Watts TLP (1989). Probing site configuration in patients with untreated periodontitis. A study of horizontal positional error. J Clin Periodontol 16: 529-33.

Watts TLP (1989). Visual and tactile observational error: Comparative probing reliability with recession and cementoenamel junction measurements. Community Dent Oral Epidemiol 17: 310-2.

Williams CA, Winkler JR, Grassi M, Murray PA (1990). HIV-associated periodontitis complicated by necrotizing stomatitis. Oral Surg Oral Med Oral Pathol 69: 351-5.

Winkler JR (1995). Pathogenesis of HIV-associated periodontal disease: What's known and what isn't. In: Greenspan JS, Greenspan D, Eds. Oral manifestations of HIV infection. Quintessence Publishing Co, Inc. Chicago, pp 263-72.

Winkler JR, Grassi M, Murray PA (1988). Clinical description and etiology of HIVassociated periodontal diseases. In: Robertson PB, Greenspan JS, Eds. Perspectives on Oral Manifestations of AIDS. PSG Publishing Company, inc. Littleton, pp 49-70.

Winkler JR, Grassi M, Williams CA, Murray PA (1988). Clinical documentation of HIV associated periodontitis and gingivitis (Abstract). 4th Intermational Conference on AIDS, Stockholm.

Winkler JR, Herrera C, Westenhouse J, Robinson P, Hessol N, Buchbinder S, Greenspan JS, Katz MH (1992). Periodontal disease in HIV-infected and seronegative homosexual and bi-sexual men. AIDS 6: 1041-3. 
Winkler JR, Murray PA (1987). A potential intra-oral expression of AIDS may be rapidly progressive periodontitis. J Calif Dent Assoc. 15: 20-4.

Winkler JR, Murray PA, Grassi M, Hammerle C (1989). Diagnosis and management of HIV-associated periodontal lesions. J. Am. Dent. Assoc. 119 (Suppl.): 25S-34S.

Winkler JR, Murray PA, Greenspan D, Greenspan JS (1986). Periodontal disease of male homosexuals as related to AIDS virus infection (Abstract). 2nd International Conference on AIDS, Paris,

Winkler JR, Murray PA, Hammerle C (1989). Gangrenous Stomatitis in AIDS (Letter). Lancet 2: 108.

Yeung SCH, Stewart GJ, Cooper DA, Doungkamol S (1993). Progression of periodontal disease in HIV seropositive patients. J Periodontol 64: 651-7.

Yoneyama T, Okamoto H, Lindhe J, Socransky SS, Haffajee AD (1988). Probing depth, attachment loss and gingival recession. J Clin Periodontol 15: 581-91.

Zakarian A, Andriolo M, Abel S, McGivern T (1988). HIV-related ulcerative gingivitis and periodontitis (Abstract). 4th International Conference on AIDS., Stockholm,

Zambon JJ, Reynolds H, Smutko J, Sabers S, Grbic J, Mitchell-Lewis D, Phelan J, Lamster I (1995). Are Unique bacterial pathogens involved in HIV-associated periodontal diseases? In: Greenspan JS, Greenspan D, Eds. Oral manifestations of HIV infection. Quintessence Publishing Co, Inc. Chicago, pp 257-62.

Zambon JJ, Reynolds HS, Genco RJ (1990). Studies of the subgingival microflora in patients with acquired immunodeficiency syndrome. J Periodontol 61: 699-704.

Zon LI, Groopman JE (1988). Hematologic manifestations of the human immune deficiency virus (HIV). Semin Hematol 25: 208-18. 
Slide

Please answer all seven questions and make a diagnosis for this slide.

1 Is the marginal gingiva

(Circle one answer only)

2 Is the attached gingiva

(Circle one answer only)
Fiery red?

Other (Please specify).

Diffuse red?

Other (Please Specify)
Red?

Punctate red? Normal?

3 Is there a well defined red band on the gingiva?

YES

NO

$4 \quad$ Is the gingiva swollen or enlarged?

YES

NO

$5 \quad$ Is there spontaneous bleeding?

YES

NO

6

Is there

increased probing depth?

observable attachment loss (recession)?

YES

NO

YES

NO

7

Is there ulceration of

the marginal gingiva? the attached gingiva? the mucosa?

YES

YES

NO

YES

NO

NO

YES

NO

$9 \quad$ What is your diagnosis?

(Circle one letter next to the answer which corresponds to your diagnosis or write another diagnosis in the space provided.

Please make a firm diagnosis)
a) A healthy periodontium
b) No gingivitis but signs of inter-dental attachment loss
c) No gingivitis but signs of generalised attachment loss
d) Conventional gingivitis
e) ANUG (NUG)
f) Conventional periodontitis with localised areas of inter-dental attachment loss
g) Conventional periodontitis with generalised attachment loss
h) HIV-Gingivitis without ulceration
i) HIV-Gingivitis with ulceration
j) HIV-Periodontitis without ulceration
k) HIV-Periodontitis with ulceration
l) Necrotising periodontitis/ Necrotising stomatitis
m) HIV-gingivitis with ulceration super-imposed on conventional periodontitis
n) HIV-gingivitis without ulceration super-imposed on conventional periodontitis
o) ANUG super-imposed on conventional periodontitis
p)
Other. 
APPENDIX 2. FREQUENCY OF DIAGNOSES IN ORIGINAL. CONTROL AND STUDY GROUPS.

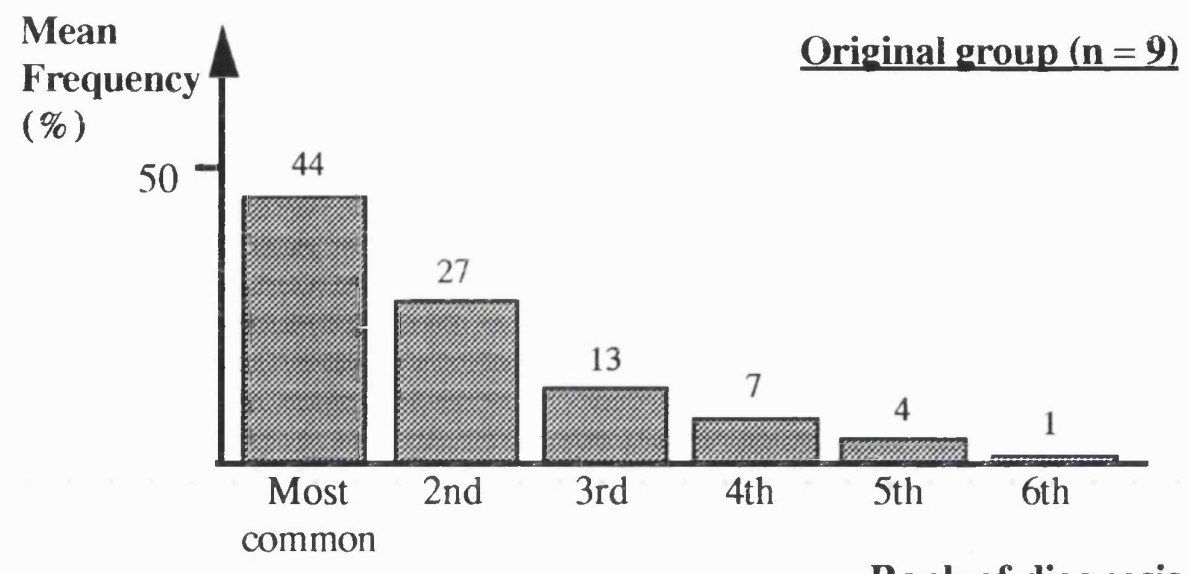

Rank of diagnosis
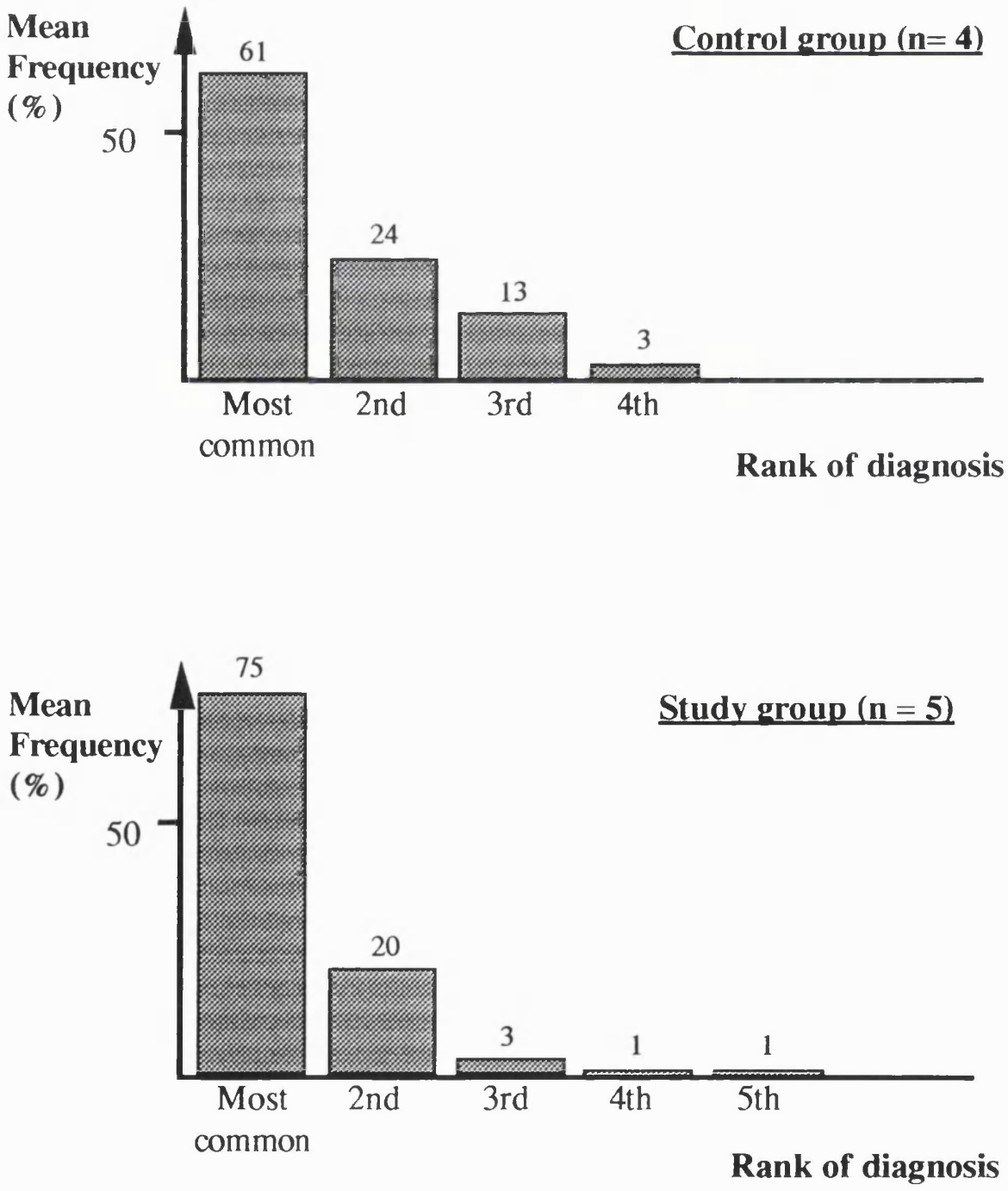


\section{Recruitment procedure for the research project 'HIV and periodontal health'.}

1 During visit physician identifies flagged notes and other gay men and suggests participation.

\section{NB Exclusion criteria}

i. People unwilling to participate.

ii. People with health problems precluding periodontal examination (e.g. history of heart valve disease or bleeding disorders).

iii. People with health problems which predispose to periodontal diseases (e.g. diabetes, acute leukaemia, epanutin or cyclosporin use)

iv. People with a history of injected recreational drug use in the last year.

v. People who have already been examined as part of this study.

Gives known HIV+ve men leaflet INFO P

Gives other gay men leaflet INFO $\mathrm{O}$ (Don't forget HIV-ves please)

Patient told to ask for Laurie after consultation.

2 Laurie explains nature of study and what participation involves.

Informs that participants will receive gift as a way of saying 'thank you'.

3 Laurie consents participants: CONSENT P for known HIV+ves CONSENT $O$ for all others

$4 \quad$ Laurie administers questionnaire and provides pencil

5 Laurie records in log book: Date

Participant's study number

Patient number at clinic

Name used by patient at clinic

6 Participant returns with questionnaire

7 Laurie checks questionnaire for completeness including patient number, participant number, date and data.

8 Laurie gives participant label with study number to give to examiner.

9 Laurie sends participant for examination.

MUST TELL EVERY PARTICIPANT NOT TO DISCUSS WHETHER THEY HAVE BEEN TESTED FOR HIV UNTIL EXAMINATION IS COMPLETE. 
Patient Number

Date

Hospital Number

\section{HIV and Periodontal Health}

This questionnaire is designed to collect information about things which might affect the health of your gums.

All the information will be treated as confidential. No one will have access to this information except the researchers. All information collected will be maintained in such a way as to keep it as confidential as possible.

Please read each question and tick the number that fits your answer best. Don't spend too much time answering the questions. You should not need to take more than 5-10 minutes over the whole questionnaire.

If you have any problems please ask.

THANK YOU FOR YOUR HELP

SECTION 1. First of all we would like to know a little about you.

1 What is your age?

$\frac{2 \quad \text { What is your occupation? }}{\text { (or your previous occupation) }}$

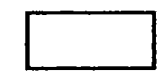

$\frac{2 \quad \text { What is your occupation? }}{\text { (or your previous occupation) }}$

$3 \quad$ How would you describe your ethnic background?
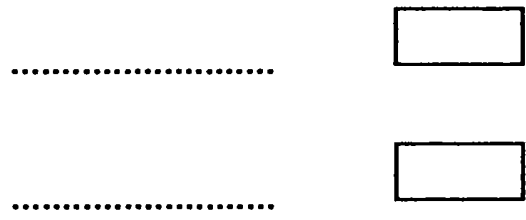

1 African

2 Afro-Caribbean

3 Asian

4 South-East Asian

5 White European

6 White non-European

7 Other (Please give details)

$4 \quad$ Are you right or left handed?

Right $1 \quad$ Left 2

$5 \quad$ What is the reason for your visit today

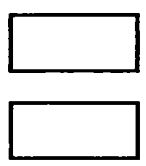

1 A routine check or because your doctor wanted to monitor your progress

2 Problems with your mouth

3 Problems with your gums

4 Problems with a different part of your body

5 Other (Please give details) 
SECTION 2. In this section we would like to find out how often you visit the dentist and whether you have had any problems with your mouth.

$\underline{6}$ How often have you been to the dentist in the past?

$\begin{array}{ll}\text { Regular attender } & 1 \\ \text { Occasional attender } & 2 \\ \text { Never or only if in pain } & 3\end{array}$

$7 \quad$ How long is it in years since your last visit to a dentist?

1

3

(Tick only one answer) Up to one year ago 1

Between one and two years ago 2

Between two and three years ago 3

Between three and four years ago 4

Between four and five years ago 5

More than five years ago 6

Never visited a dentist $\quad 7$

$8 \quad$ Are your gums causing you any of the following problems at the moment?

(Tick as many answers as you need)

$\begin{array}{lc}\text { Sensitivity } & 1 \\ \text { Painful gums } & 2 \\ \text { Receding gums } & 3 \\ \text { Bleeding gums } & 4 \\ \text { Loose teeth } & 5\end{array}$

Other Problems (Please specify in the space below)

$9 \quad$ Have you had any of the following problems with your gums in the past 2 months?

(Tick as many answers as you need)

$\begin{array}{lc}\text { Sensitivity } & 1 \\ \text { Painful gums } & 2 \\ \text { Receding gums } & 3 \\ \text { Bleeding gums } & 4 \\ \text { Loose teeth } & 5 \\ \text { Other Problems (Please specify in the space below) }\end{array}$

10 Have you used Corsodyl Mouthrinse in the last two weeks?

$\begin{array}{ll}\text { Yes } & 1 \\ \text { No } & 2\end{array}$




\section{APPENDIX 4. CONTINUED}

\section{SECTION 3 Smoking and Drinking}

Many things affect the health of people's gums. We would like to ask you some questions about things which might affect your gums

11 Do you smoke tobacco now?

Yes 1

No 2

For Office

Use only

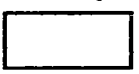

\section{If you have answered No to this question please go to question 18}

12 How many manufactured cigarettes do you smoke per day?

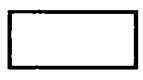

and/or

13 About how many ounces of tobacco do you use each week

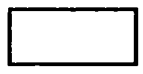
for hand-rolled cigarettes?

$\underline{14}$

Do you smoke cigars?

Yes 1

No 2

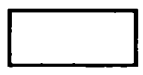

15 If yes, how many cigars per week
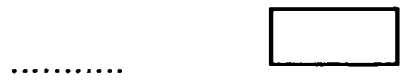

16

Do you smoke a pipe?

Yes 1

No 2

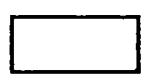

17 About how many ounces of tobacco do you smoke each week?

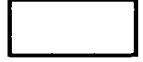

18 If you are not a smoker at present did you smoke in the past?

$$
\text { Yes } 1 \quad \text { No } 2
$$

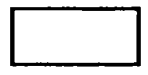

19 In the past 12 months have you taken an alcoholic drink:

(Tick one answer only)

$\begin{array}{ll}\text { Almost daily } & 2 \\ \text { Once or twice a week } & 3 \\ \text { Once or twice a month } & 4 \\ \text { Special occasions only } & 5 \\ \text { No } & 6\end{array}$

20 Think back five years, how often did you take an alcoholic drink then?

A lot more than now

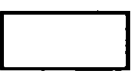

A bit more than now

About the same as now

A bit less than now

A lot less than now 


\section{APPENDIX 4. CONTINUED}

\section{SECTION 4 Drug use}

This page enquires about the use of several drugs. The effects of these drugs on the health of your gums is not known. It is hoped that this line of research will shed light on any effects they may have.

$21 \quad$ Have you ever smoked or inhaled any other drugs?

For example; Cannabis (Marijuana, dope, grass), Amyl nitrate (Poppers), Amphetamines (Speed), Cocaine (Coke), Heroin/Opium (Smack)

Yes $1 \quad$ No 2

If you have answered NO to this question go to the bottom of the page.

22 Do you smoke cannabis now?

23 Did you smoke cannabis in the past?

24 Do you use Amyl Nitrate (Poppers) now?

$\underline{25}$ $\underline{26}$

$\underline{29}$

$\underline{30}$

$\underline{3}$

$\underline{32}$

$\underline{33}$

If you do please specify

$\underline{34}$ Did you smoke or inhale any other drugs in the past?

Yes 1
No 2

No 2

No 2

No 2

No 2

No 2

No 2

No 2

No 2

No 2

No 2
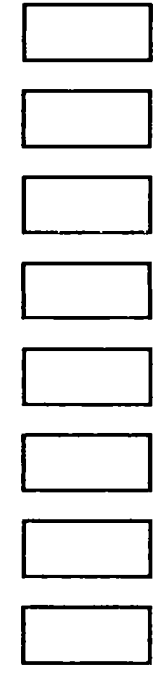

Yes 1

Yes 1

Yes 1
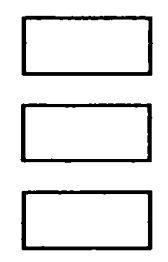
If you did please specify

No 2

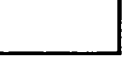

Again, thank you for taking part. 
APPENDIX 5. SHEET FOR MEDICAL DATA IN CROSS-SECTIONAL STUDY

Medical Sheet

Hospital Number.
Partic. No

DateExam

$\begin{array}{ll}\text { HIV Status } & \text { Untested (1), -ve } \\ \begin{array}{l}\text { Clinical Stage } \\ \text { (HIV+ve only) }\end{array} & \begin{array}{l}\text { Acute/Asympt/PGL } \\ \text { AIDS (C), Other }\end{array} \\ \begin{array}{l}\text { Haematology } \\ \text { (x 109/L) }\end{array} & \text { CD4 } \\ \text { (Within 6 weeks) } & \text { CD8 } \\ & \text { CD4/CD8 } \\ & \text { Lymphocytes } \\ & \text { Neutrophils }\end{array}$

Medications (Y/N)
Current

Septrin

Fansidar

Pentamidine

Dapsone

Nystatin

Amphotericin

Miconazole

Ketoconazole

Fluconazole

Itraconazole

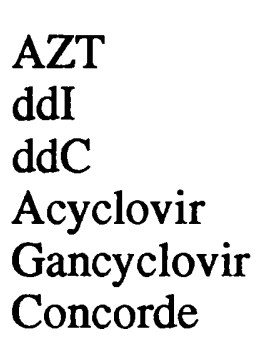

Prednisolone

Azathioprine

Others
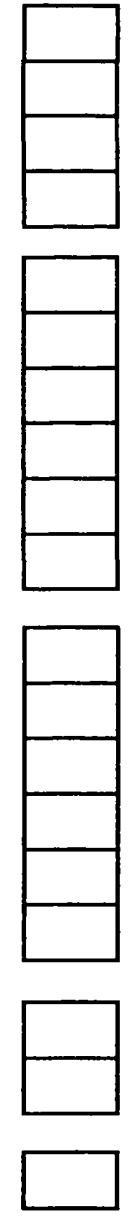

(3)
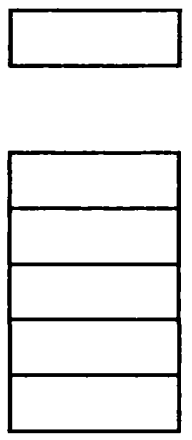

Last $3 / 12$

Septrin

Fansidar

Pentamidine

Dapsone

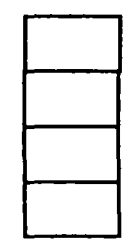

Nystatin

Amphotericin Miconazole Ketoconazole Fluconazole Itraconazole

AZT

ddI ddC Acyclovir Gancyclovir Concorde

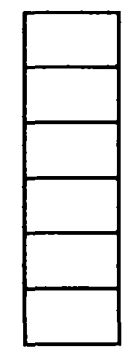

Prednisolone Azathioprine

Others
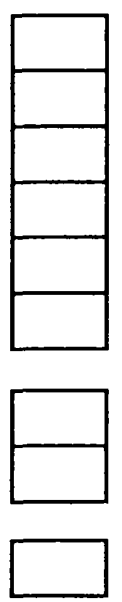


\title{
APPENDIX 6. INFORMATION SHEET FOR KNOWN HIV POSITIVE PARTICIPANTS IN CROSS-SECTIONAL STUDY
}

\section{UNIVERSITY COLLEGE HOSPITAL}

\author{
Information on the research project
}

\section{HIV infection and periodontal health}

\begin{abstract}
Peter Robinson of the Dental Department at University College Hospital is carrying out research into the gum problems experienced by people with HIV infection. This is done by comparing the gums of people with HIV against the gums of people without HIV. This research is of value since it may shed light on the type of problems people with HIV get, how many people get them, what causes them, how to prevent and how to treat them.

If you take part you will help by:

i) completing a short questionnaire to find out about any problems you may have with your mouth and things which might affect the health of your gums

ii) having photographs being taken of your mouth

iii) having an examination of your mouth
\end{abstract}

The whole thing should take about half an hour of your time. To cut down on the time you need to spend with us we would like to collect information on your general health from your medical records.

There are no risks in participating and there should be no more discomfort than in a dental check up. Should there be any untoward effects of taking part in the study you will be fully recompensed. However, there are some people who may be more susceptible to gum diseases or who it is best not to examine for this study. Please read the list of conditions below:

people who have had Rheumatic fever, Diabetes or Leukaemia people with Prosthetic heart valves people who have taken epanutin or cyclosporin medicines.

If you fall into any of these categories please tell the person who gave you this form. Sorry, but your gums are not suitable for this research!

All information gathered in the study will be treated as confidential. No one will have access to this information except the researchers. Neither your name nor anything that identifies you will be used in any reports of this study. All information collected will be maintained in such a way as to keep it as confidential as possible.

Taking part in this study is voluntary. You can refuse to take part at any time.

A small pack of personal hygiene products manufactured by SmithKline Beecham will be offered to every one who participates.

If you have any questions about this project please ask Peter Robinson (the dentist) or the Research Assistant (who gave you this information sheet). 


\title{
APPENDIX 7. INFORMATION SHEET FOR PARTICIPANTS OF NEGATIVE AND UNKNOWN HIV STATUS IN CROSS-SECTIONAL STUDY
}

\section{UNIVERSITY COLLEGE HOSPITAL}

\author{
Information on a research project
}

\begin{abstract}
Although we know that you have not been found to have HIV infection we would like to invite you to take part in a research project on HIV infection and periodontal diseases.

Peter Robinson of the Dental Department at University College Hospital is carrying out research into the gum problems experienced by people with HIV infection. This is done by comparing the gums of people with HIV against the gums of people without HIV. This research is of value since it may shed light on the type of problems people with HIV get, how many people get them, what causes them, how to prevent and how to treat them.
\end{abstract}

If you take part you will help by:

i) completing a short questionnaire to find out about any problems you may have with your mouth and things which might affect the health of your gums

ii) having photographs being taken of your mouth

iii) having an examination of your mouth

The whole thing should take about half an hour of your time. To cut down on the time you need to spend with us we would like to collect information on your general health from your medical records.

There are no risks in participating and there should be no more discomfort than in a dental check up. Should there be any untoward effects of taking part in the study you will be fully recompensed. However, there are some people who may be more susceptible to gum diseases or who it is best not to examine for this study. Please read the list of conditions below:

people who have had Rheumatic fever, Diabetes or Leukaemia people with Prosthetic heart valves people who have taken epanutin or cyclosporin medicines.

If you fall into any of these categories please tell the person who gave you this form. Sorry, but your gums are not suitable for this research!

All information gathered in the study will be treated as confidential. No one will have access to this information except the researchers. Neither your name nor anything that identifies you will be used in any reports of this study. All information collected will be maintained in such a way as to keep it as confidential as possible.

Taking part in this study is voluntary. You can refuse to take part at any time. A small pack of personal hygiene products manufactured by SmithKline Beecham will be offered to every one who participates.

We would like to stress again, no one has found you have HIV. We would like to examine you to compare your gums with those of people who have. If you have any questions about this project please ask Peter Robinson (the dentist) or the Research Assistant (who gave you this information sheet).

THANK YOU FOR YOUR TIME 


\title{
APPENDIX 8. CONSENT FORM FOR KNOWN HIV POSITIVE PARTICIPANTS IN CROSS- SECTIONAL STUDY
}

\section{UNIVERSITY COLLEGE HOSPITAL}

\author{
Consent to participate in the research project
}

\section{HIV infection and periodontal diseases}

Peter Robinson of the Dental Department of University College Hospital is carrying out research into the gum problems experienced by people with HIV infection. This is done by comparing the gums of people with HIV against the gums of people without HIV. This research is of value since it may shed light on the type of problems people with HIV get, how many people get them, what causes them, how to prevent and how to treat them.

Participation in the study involves:

i) completing a short questionnaire to find out about any problems you may have with your mouth and things which might affect the health of your gums

ii) having photographs being taken of your mouth

iii) having an examination of your mouth

The whole thing should take about half an hour of your time. To cut down on the time you need to spend with us we would like to collect information on your general health from your medical records.

All information gathered in the study will be treated as confidential. No one will have access to this information except the researchers. Neither your name nor anything that identifies you will be used in any reports of this study. All information collected will be maintained in such a way as to keep it as confidential as possible.

If you have any questions before you go on please ask the research assistant who gave you this form.

THANK YOU FOR TAKING PART

The information above has been explained to me by

and I understand what will be required of me if I take part in the study. My questions concerning this study have been answered by him/her.

I have not had any of the following illnesses: Rheumatic fever, Diabetes or Leukaemia. I do not have a Prosthetic heart valve and I have not taken epanutin or cyclosporin medicines.

I agree to participate in the research being undertaken by Peter Robinson. I understand that at any time I may withdraw from this study without giving a reason and without affecting my normal care or management.

Signed

Date

Dentist 


\title{
APPENDIX 9. CONSENT FORM FOR PARTICIPANTS OF NEGATIVE AND UNKNOWN HIV STATUS IN CROSS-SECTIONAL STUDY
}

\section{UNIVERSITY COLLEGE HOSPITAL}

\author{
Consent to participate in a research project
}

\begin{abstract}
Although we know that you have not been found to have HIV infection we would like to invite you to take part in a research project on HIV infection and periodontal diseases.

Peter Robinson of the Dental Department of University College Hospital is carrying out research into the gum problems experienced by people with HIV infection. This is done by comparing the gums of people with HIV against the gums of people without HIV. This research is of value since it may shed light on the type of problems people with HIV get, how many people get them, what causes them, how to prevent and how to treat them.
\end{abstract}

Participation in the study involves:

i) completing a short questionnaire to find out about any problems you may have with your mouth and things which might affect the health of your gums

ii)

having an examination of your mouth

The whole thing should take about half an hour of your time. To cut down on the time you need to spend with us we would like to collect information on your general health from your medical records.

All information gathered in the study will be treated as confidential. No one will have access to this information except the researchers. Neither your name nor anything that identifies you will be used in any reports of this study. All information collected will be maintained in such a way as to keep it as confidential as possible.

We would like to stress again, no one has found you have HIV. We would like to examine you to compare your gums with those of people who have. If you have any questions about this project please ask Peter Robinson (the dentist) or the Research Assistant (who gave you this information sheet). If you have any questions before you go on please ask the research assistant who gave you this form.

THANK YOU FOR TAKING PART

The information above has been explained to me by

and I understand what will be required of me if I take part in the study. My questions concerning this study have been answered by him/her.

I have not had any of the following illnesses: Rheumatic fever, Diabetes or Leukaemia. I do not have a Prosthetic heart valve and I have not taken epanutin or cyclosporin medicines.

I agree to participate in the research being undertaken by Peter Robinson. I understand that at any time I may withdraw from this study without giving a reason and without affecting my normal care or management.

Signed

Date

Dentist 
APPENDIX 10. PROPORTIONS OF TOOTH SURFACES IN MEN WITH_AND_WITHOUT HIV INFECTION WITH PLAOUE SCORES OF $0,1,2$ AND 3.

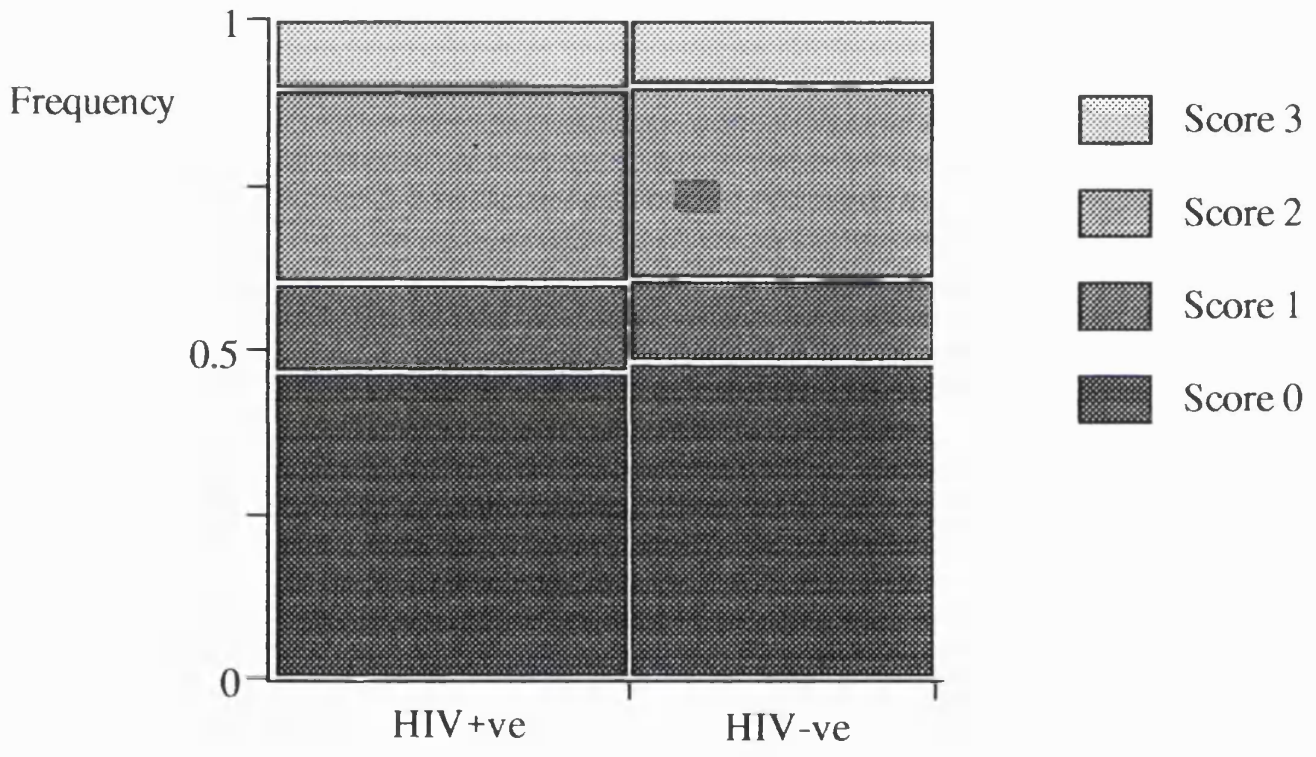

Status

\begin{tabular}{|c|cc|}
\hline Score* & $\begin{array}{c}\text { HIV +ve (\%) } \\
\mathbf{n}=\mathbf{8 4 6 1}\end{array}$ & $\begin{array}{c}\text { HIV-ve (\%) } \\
\mathbf{n}=\mathbf{7 1 5 7}\end{array}$ \\
\hline $\mathbf{3}$ & 10.5 & 9.7 \\
$\mathbf{2}$ & 29.4 & 29.5 \\
$\mathbf{1}$ & 13.6 & 12.6 \\
$\mathbf{0}$ & 46.6 & 48.3 \\
\hline
\end{tabular}

* Plaque index of Silness and Löe (1964). 
APPENDIX 11. FREQUENCY OF ATTACHMENT LOSS OF 4 MM OR MORE BY TOBACCO USE IN HIV-VE AND HIV + VE MEN

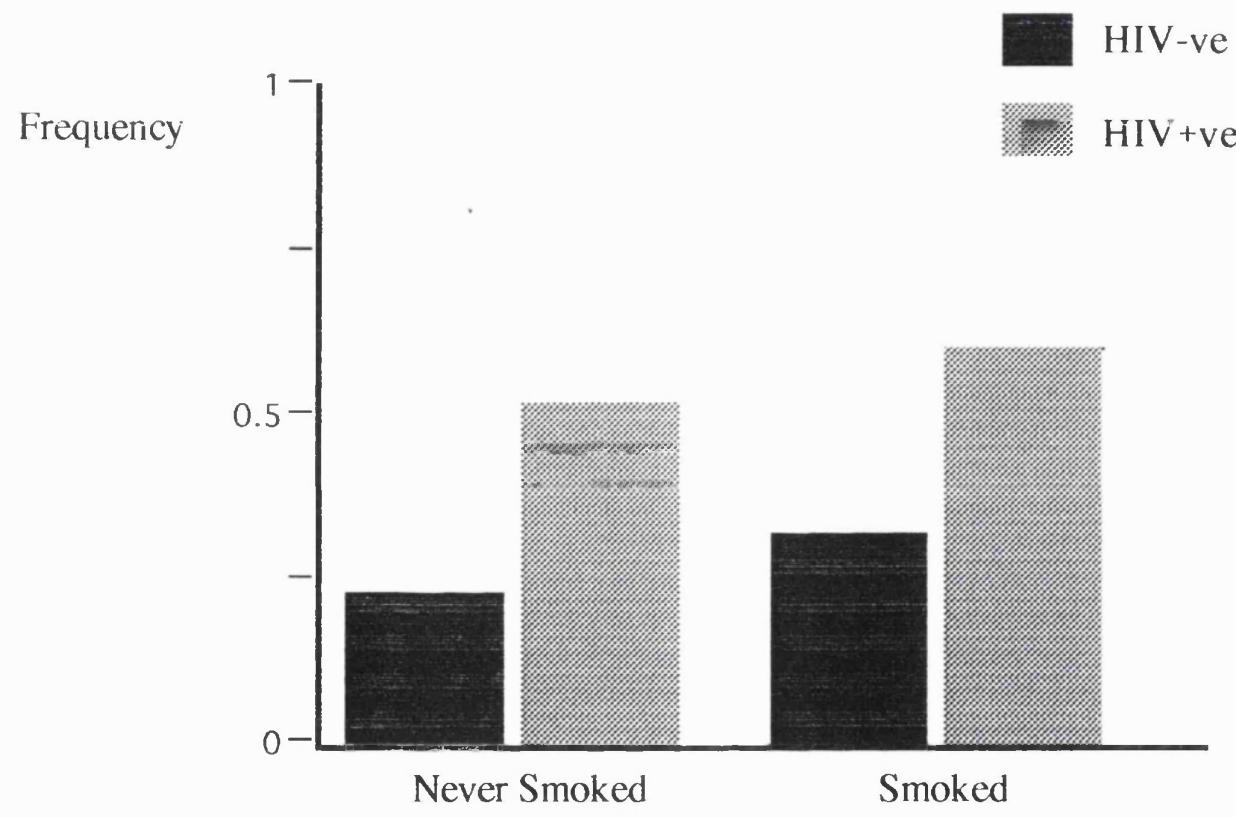

Tobacco use 
APPENDIX 12. SEVERITY OF ATTACHMENT LOSS BY PROPORTION OF SURFACES WITH PLAQUE IN HIV+VE AND -VE MEN.

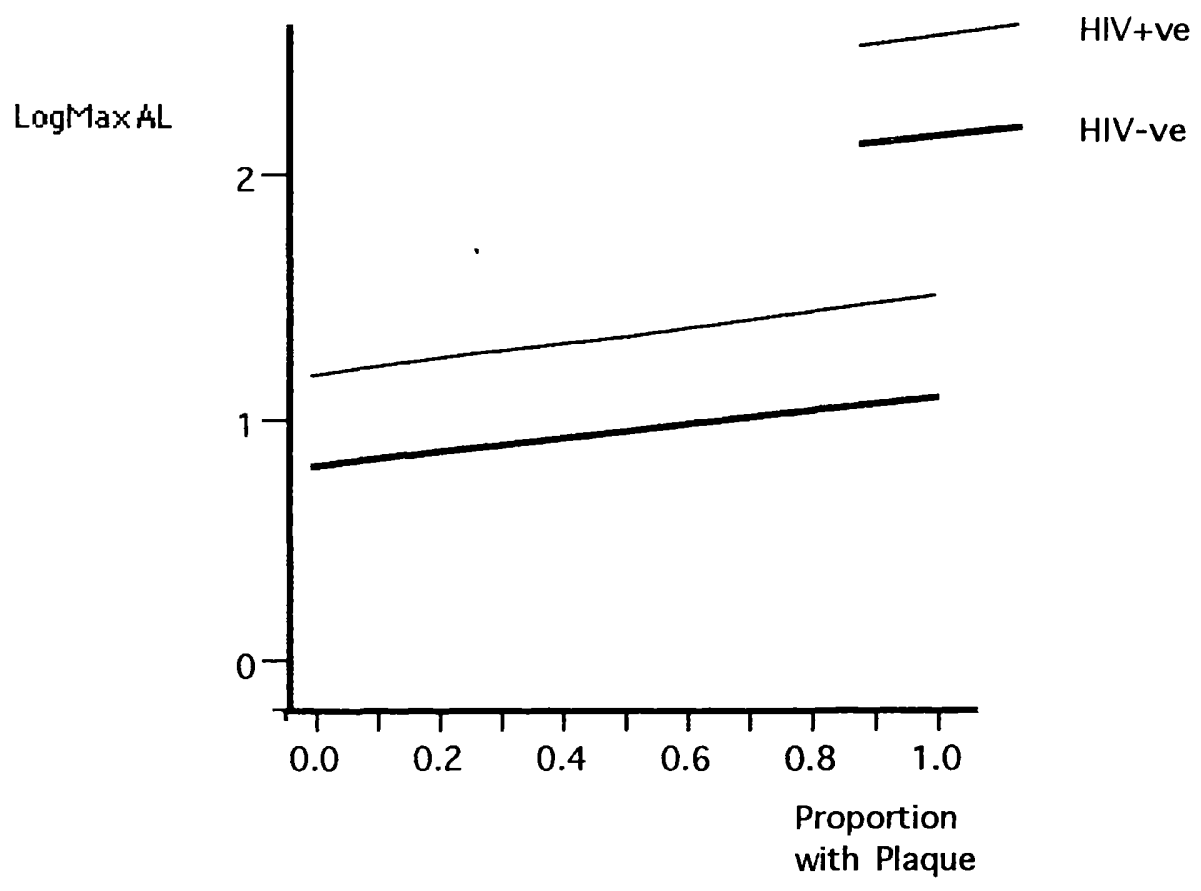


APPENDIX 13. EXTENT OF POCKETING IN MEN WITH AND WITHOUT HIV INFECTION BY AGE.

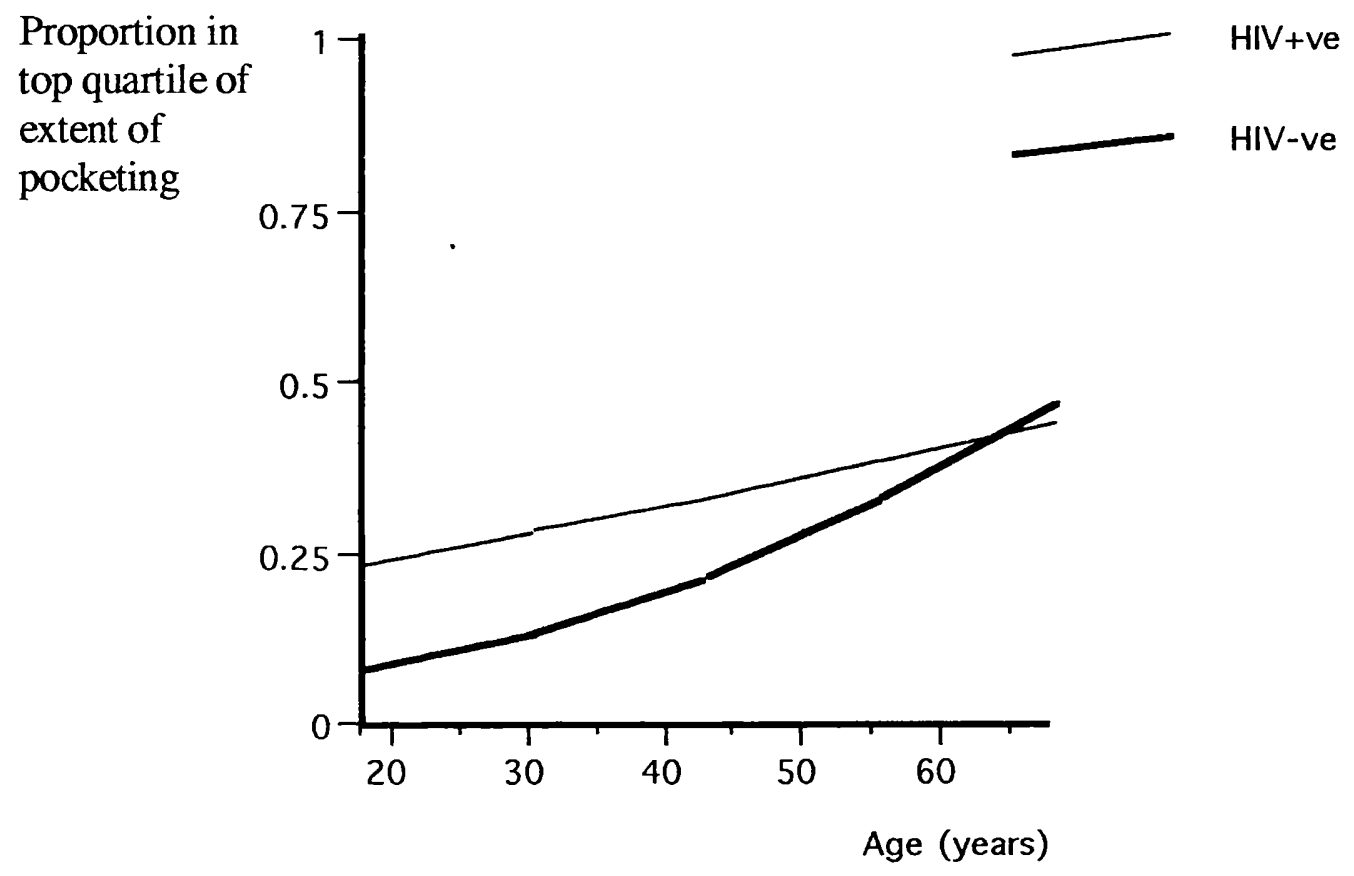


APPENDIX 14. PREVALENCE OF ATTACHMENT LOSS AND PROBING DEPTHS $\geq 4 \mathrm{MM}$ BY HIV STATUS AND CD4 COUNT

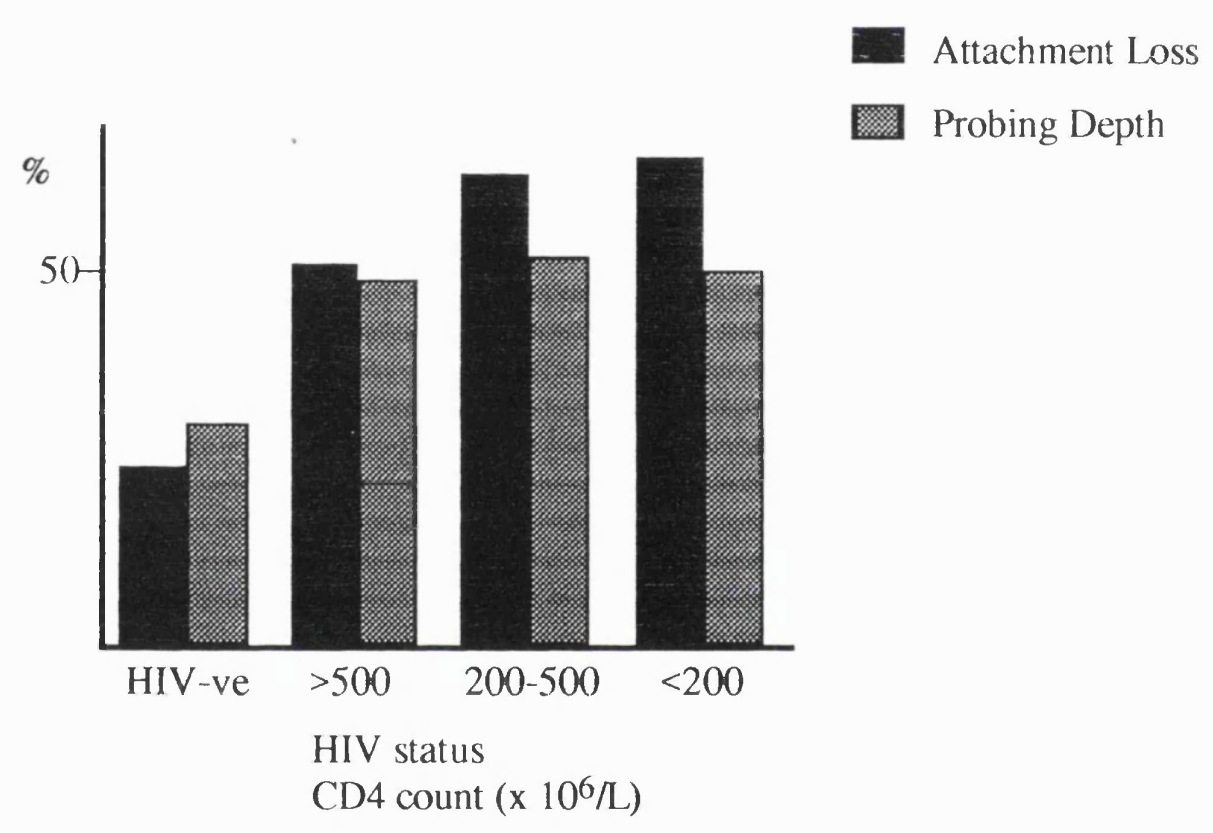


APPENDIX 15. SEVERITY OF_CONVENTIONAL GINGIVITIS AND PUNCTATE ERYTHEMA OF: THE ATTACHED GINGIVA BY HIV STATUS AND PRESENCE OF ORAL CANDIDIASIS

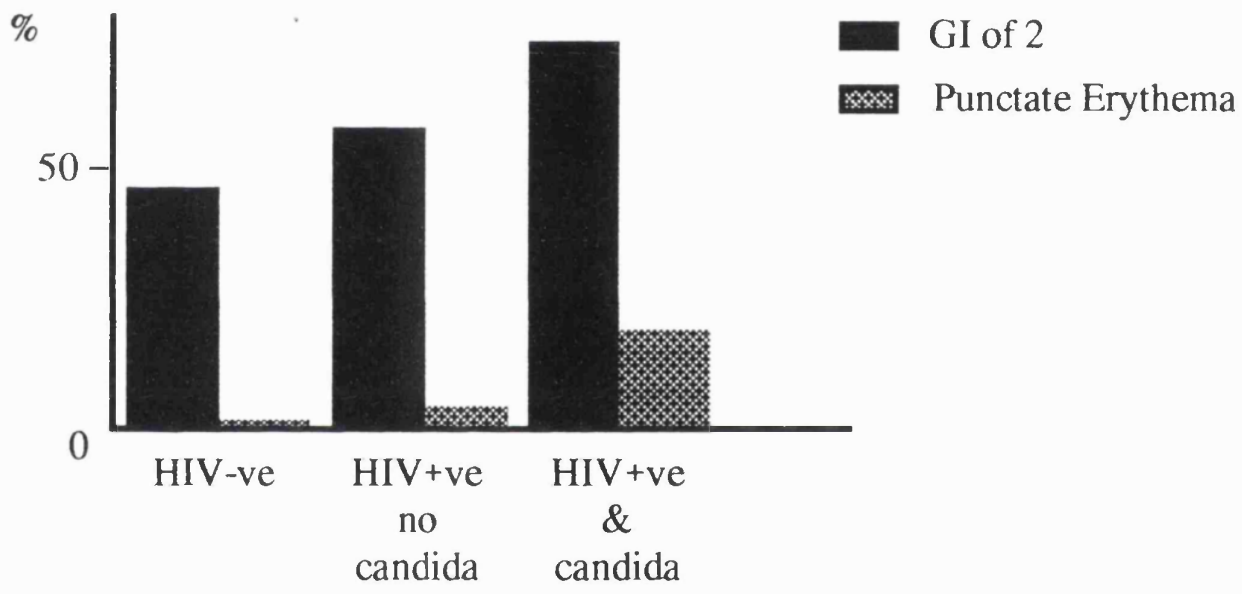


APPENDIX 16. LINEAR REGRESSION OF NUMBER OF ULCERATED SITES IN FIRST EPISODE OF GINGIVAL ULCERATION BY AGE.

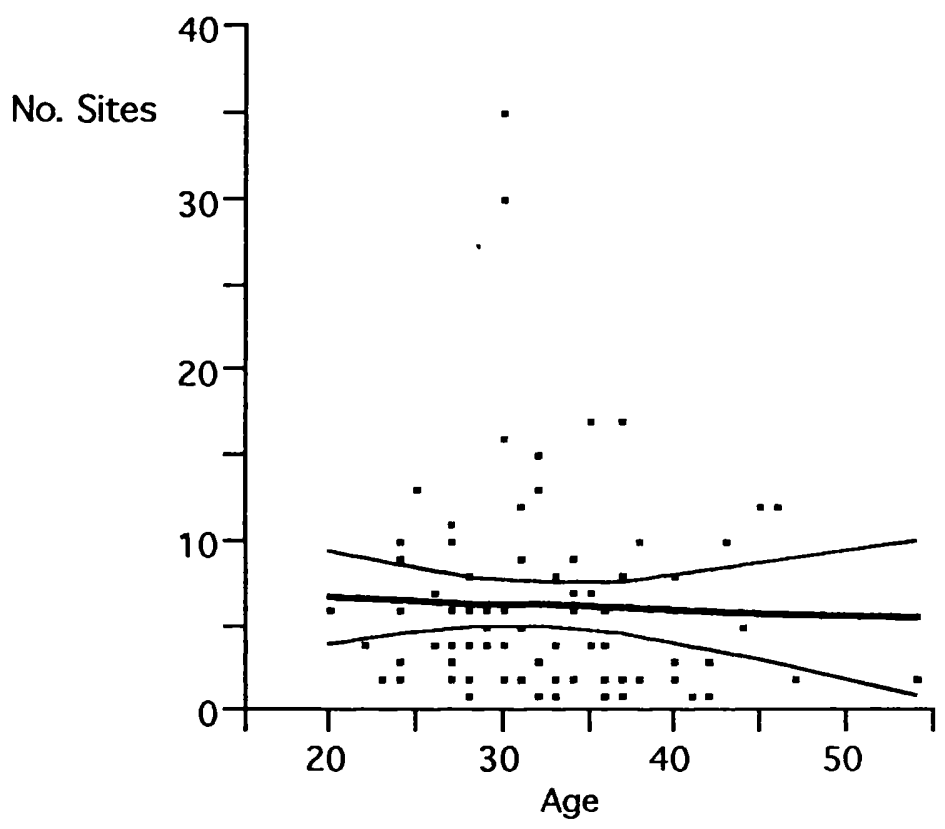

三 Linear Fit and 95\% confidence interval

Observations 85

Parameter Estimates

Term b

Intercept

7.5

se

P

Age

0.0

3.3

0.1

0.717

$\mathrm{b}=$ Regression coefficient

se $=$ standard error 
APPENDIX 17. LINEAR REGRESSION OF NUMBER OF ULCERATED SITES IN FIRST EPISODE OF GINGIVAL ULCERATION BY NEUTROPHIL COUNT

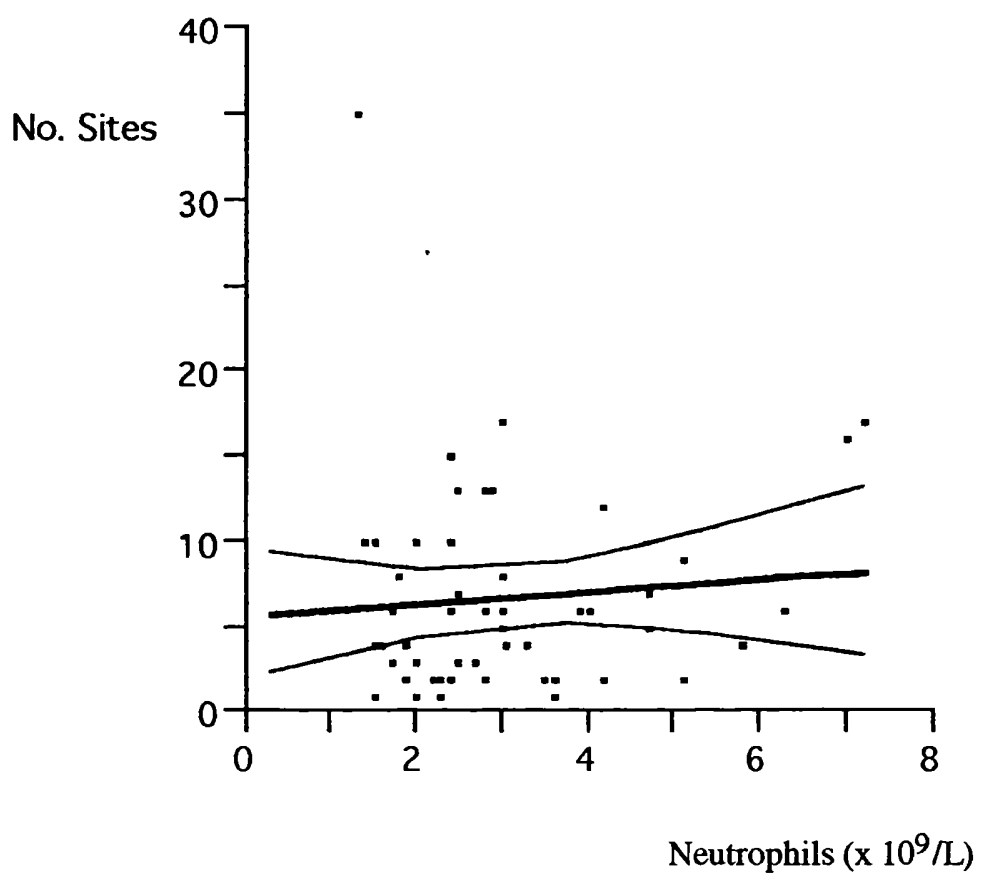

三 Linear Fit and 95\% confidence interval

Observations 52

Parameter Estimates

Term

Intercept

Neutrophils

$\mathrm{b}=$ Regression coefficient

se $=$ standard error

\section{b}

5.7

0.4

0.6

\begin{tabular}{ll}
\hline se & $\boldsymbol{P}$ \\
1.9 & \\
0.6 & 0.529 \\
\hline
\end{tabular}




\begin{tabular}{|l|cc|l|}
\hline & $\begin{array}{c}\text { Cases } \\
(\mathrm{n}=89)\end{array}$ & $\begin{array}{c}\text { Controls } \\
(\mathrm{n}=269)\end{array}$ & \multicolumn{1}{|c|}{$P$} \\
\hline Mean age (y) (SD) & $32.4(6.5)$ & $361(8.2)$ & $<0.001, \mathrm{t}$ test \\
Mean neutrophil count x 10\%/L(SD) & $3.03(1.5)$ & $2.5(1.3)$ & $0.013, \mathrm{t}$ test \\
Median CD4 count x 109/L(95\% CR) & $320(13-1118)$ & $270(10-835)$ & 0.166, t test* \\
\hline AIDS (\%) $\dagger$ & 27.0 & 43.5 & 0.033 , Chi sq. \\
Tobacco use (\%) $\dagger$ & 66.7 & 56.6 & 0.128 , Chi sq. \\
Oral candidiasis (\%) $\dagger$ & 56.7 & 27.3 & $<0.001$, Chi sq. \\
Hairy leukoplakia (\%) $\dagger$ & 44.8 & 33.3 & 0.080 , Chi sq. \\
Kaposi's sarcoma (\%) $\dagger$ & 1.5 & 5.9 & 0.134, Chi sq. \\
Mucosal ulceration (\%) $\dagger$ & 4.5 & 4.5 & 0.995 , Chi sq. \\
\hline
\end{tabular}

*Using the Root CD4 transformation.

$\dagger$ Data are expressed as proportions of the total for whom data were available. 
APPENDIX 19. BEST FORWARD STEPWISE REGRESSION MODEL FOR GINGIVAL ULCERATION EXCLUDING PARTICIPANTS WHO WERE NOT HOMOSEXUAL MEN

\begin{tabular}{|lllr|}
\hline$R^{2}=0.108$ & & & \\
& \multicolumn{1}{l}{} & & \multicolumn{1}{c|}{} \\
Intercept & 0.712 & 0.730 & 0.329 \\
Age & -0.059 & 0.021 & 0.005 \\
Candidiasis & 0.682 & 0.149 & $<0.001$ \\
Not AIDS & 0.360 & 0.156 & 0.021 \\
\hline
\end{tabular}

$b=$ regression coefficient

se $=$ standard error

$R^{2}=$ proportion of variation explained by the model 
APPENDIX 20. DIAGNOSTIC CRITERIA FOR HIV ASSOCIATED PERIODONTAL DISEASES USED BY RILEY ET AL. (1992).

\section{HIV-associated gingivitis}

Intensely red free gingival margins, no indication of attachment loss nor pockets greater than $3 \mathrm{~mm}$.

Acute Necrotising Ulcerative Gingivitis

Sudden onset painful white ulcerated lesion with pseudomembrane, beginning at the interdental papillae but no bone loss, foetor ex ore was usually present.

Necrotising Ulcerative Periodontitis

Very similar to ANUG but with loss of periodontal attachment and alveolar bone. 
APPENDIX 21. DIAGNOSTIC CRITERIA FOR NUG USED BY THOMPSON ET AL. (1992)

Interproximal necrotic ulcers usually covered with yellowish white or greyish layer of fibrinopurulent exudate. 
APPENDIX 22. DIAGNOSTIC CRITERIA FOR HIV ASSOCIATED PERIODONTAL DISEASES OF THE USA ORAL AIDS COLLABORATIVE GROUP (GREENSPAN ET AL. 1992).

\section{HIV-associated gingivitis (Presumptive)}

Erythematous marginal gingival band that can extend into adjacent attached and alveolar mucosa

(Definitive criterion demands that lesion does not respond to removal of dental plaque and calculus and oral hygiene measures).

\section{HIV-associated periodontitis (necrotising periodontitis) (Presumptive)}

Severely destructive lesion characterised by soft tissue ulceration and necrosis with rapidly progressive, non-self-healing loss of periodontal attachment and bone. Deep pain and spontaneous bleeding are or may have been associated with the lesion.

(Definitive criterion requires documented progression of rapid tissue loss (within 4 weeks) and exclusion of other causes of periodontal (soft and hard tissue) destruction).

\section{Necrotising stomatitis}

Localised acute, painful ulcero-necrotic lesion of oral mucosa that exposes underlying bone or penetrates or extends into contiguous tissues. Margins are sharply defined and undermined, and specific laboratory studies including biopsy are not useful in identifying etiologic agents or processes. 
APPENDIX 23. DIAGNOSTIC CRITERIA FOR HIV ASSOCIATED PERIODONTAL DISEASES OF THE EC-CLEARINGHOUSE ON ORAL PROBLEMS RELATED TO HIV INFECTION.(1993)

\section{Linear Gingival Erythema (Presumptive)}

A distinct fiery red band along the margin of the gingiva. The amount of erythema is disproportionately intense for the amount of plaque seen. No ulceration is present and there is no evidence of pocketing or attachment loss.

\section{Necrotising ulcerative gingivitis (Presumptive)}

Destruction of one or more interdental papillae. In the acute stage of the process ulceration, necrosis and sloughing may be seen, with ready haemorrhage and characteristic foetor.

\section{Necrotising (ulcerative) periodontitis (NUP) (Presumptive)}

Periodontitis characterised by soft tissue loss as a result of ulceration or necrosis. Exposure, destruction or sequestration of bone may be seen, and the teeth may become loosened. Pain may be a prominent feature. (Destruction might extend over the muco-gingival junction and the disease could be seen with ulceration during an active phase or without ulceration during a less active phase)

\section{Necrotising ulcerative stomatitis}

Localised acute painful ulceronecrotic lesion of the oral mucosa that exposes underlying bone or penetrates or extends into contiguous tissues. These lesions may extend from areas of necrotising periodontitis. 
APPENDIX 24. DIAGNOSTIC CRITERIA FOR HIV ASSOCIATED PERIODONTAL DISEASES USED BY GRBIC ET AL. (1995).

\section{Linear Gingival Erythema}

Distinct, complete erythematous band present from papilla to adjacent papilla and extending at least $2 \mathrm{~mm}$ from the gingival margin.

\section{Necrotising Ulcerative Gingivitis}

At least one papilla appeared ulcerated or had a crater-like appearance and the patient had moderate to severe pain or a history of pain in the area within the last 6 months.

Necrotising Ulcerative Periodontitis

As above in addition necrotic alveolar bone was exposed or there was evidence of at least 3 $\mathrm{mm}$ of probing attachment loss in the interproximal area. 
APPENDIX 25. PUBLICATIONS, PUBLISHED ABSTRACTS AND PRESENTATIONS AT MAJOR SCIENTIFIC MEETINGS IN SUPPORT OF THIS WORK.

\section{Refereed papers}

Robinson PG. Periodontal Diseases and HIV infection: A Review of the Literature. J. Clin. Periodontol. 1992;19:609-14.

Robinson P, Winkler J, Palmer G, Westenhouse J, Hilton J \& Greenspan J. The diagnosis of periodontal diseases associated with HIV infection. J. Periodontol. 1994;65:236-43.

Robinson P. The diagnosis of periodontal changes associated with HIV infection. Oral Manifestations of HIV Infection. 1995. Quintessence Publishing Co, Inc. Chicago.

Robinson PG, Sheiham A, Challacombe SJ, Zakrzewska. The periodontal health of homosexual men with HIV infection: A controlled study. Oral Diseases. 1996;2:45-52

\section{Published abstracts}

Robinson P. Mucosal lesions in homosexual men attending a genito-urinary medicine clinic. J Dent Res 1993:72;121.

Robinson P \& Zakrzewska J. Gingival Ulceration associated with HIV infection. J Dent Res 1994;64:827

Robinson P, Sheiham A, Challacombe SJ, Zakrzewska JM. Periodontal attachment levels associated with HIV infection. J Dent Res 1995:74;832.

Robinson P, Challacombe SJ, Sheiham A, Zakrzewska JM. Mucosal lesions as predictors of HIV infection. J Dent Res 1995:74;454.

\section{Work presented at other meetings}

Robinson P, Winkler J, Palmer G, Westenhouse J, Hilton J \& Greenspan J. The diagnosis of periodontal diseases associated with HIV infection. 2nd International Workshop on Oral Manifestations of HIV infection. San Francisco 1993.

Robinson P. An evaluation of 83 cases of gingival ulceration associated with HIV infection. 2nd International Workshop on Oral Manifestations of HIV infection. San Francisco 1993.

Robinson P, Sheiham A, Challacombe SJ, Zakrzewska JM. Periodontal health of homosexual men with HIV infection. British Society of Oral Medicine. London, May 1994. 


\section{APPENDIX 26. GLOSSARY OF ABBREVIATIONS}

AIDS Acquired immunodeficiency syndrome

ANUG Acute necrotising ulcerative gingivitis

ARC AIDS related complex.

Atyp Atypical gingivitis (later HIV-G and LGE)

AVAP AIDS virus associated periodontitis (later HIV-P)

AZT Azidothymidine, Zidovudine

b Regression coefficient

$B$ cells B lymphocytes

BOP Bleeding on probing

CD Cluster determinant (e.g. CD4)

CDC Centers for Disease Control and Prevention

CEJ Cemento-enamel junction

cf. Compared with

CI Confidence interval

CMG Chronic marginal gingivitis

Con A Concanalvin A

CPITN Community Periodontal Index of Treatment Needs

CR Central range

ddC Dideoxycytidine

ddI Dideoxyinosine, didanosine

DNA Deoxyribonucleic acid

EBV Epstein-Barr Virus

ELISA Enzyme-linked immunosorbant assay

gp120 Glycoprotein 120

HIV Human immunodeficiency virus

HIV-G HIV-assocated gingivitis

HIV-P HIV-associated periodontitis

HIV-SGD HIV salivary gland disease

IDU Injecting drug user

KS Kaposi's sarcoma

LGE Linear gingival erythema

Log Logarithm

NHL Non-Hodgkin's lymphoma

NOS Not otherwise specified

NUG Necrotising ulcerative gingivitis

NUP Necrotising ulcerative periodontitis 


\section{APPENDIX 21. GLOSSARY CONTINUED.}

$\begin{array}{ll}\text { OHL } & \text { Oral hairy leukplakia } \\ \text { OR } & \text { Odds ratio } \\ \text { PAL } & \text { Periodontal attachment loss } \\ \text { PDI } & \text { Periodontal Disease Index } \\ \text { PI } & \text { Russell's Periodontal Index } \\ \text { PMN } & \text { Polymorphonuclear leukocyte } \\ \text { PPV } & \text { Positive predictive value } \\ R^{2} & \text { Proportion of variation explained by a regression model } \\ \text { RNA } & \text { Ribonucleic acid } \\ \text { RPP } & \text { Rapidly progressive periodontitis } \\ \text { RR } & \text { Relative risk } \\ \text { SD } & \text { Residual standard deviation } \\ \text { se } & \text { Standard error } \\ \text { Spp. } & \text { Species } \\ \text { T cells } & \text { T lymphocytes } \\ \text { TNF } & \text { Tumour necrosis factor } \\ \text { UCSF } & \text { University of California at San Francisco } \\ \text { WHO } & \text { World Health Organisation }\end{array}$




\title{
The Diagnosis of Periodontal Conditions Associated With HIV Infection
}

\author{
Peter G. Robinson, ${ }^{*}$ James $R$. Winkler, ${ }^{\dagger}$ Guy Palmer, ${ }^{\ddagger}$ Janice Westenhouse, ${ }^{\dagger}$
}

Joan F. Hilton, ${ }^{\dagger}$ and John S. Greenspan ${ }^{\dagger}$

OBJECTIVE, RELIABLE, AND VALID DIAGNOSTIC criteria are required for studies of HIVassociated periodontal conditions. A set of diagnostic criteria were devised based on a literature review and the clinical experience of the authors. Validity was assessed by comparison with clinical photographs and the criteria were evaluated and refined for ease of use and objectivity. To assess the reliability of the criteria, 9 experienced examiners were shown 20 clinical photographs accompanied by brief vignettes of clinical information. Each examiner was asked to identify signs evident in a particular area of the photograph and to record a diagnosis. Five examiners were then trained and calibrated in the use of the criteria. Finally, all 9 examiners were shown the original 20 photographs and asked to identify signs and record diagnoses. The examiners showed only fair reliability in the recognition of clinical signs, made diagnoses intuitively, and had only fair agreement on the diagnosis of periodontal diseases. The inter-examiner reliability of examiners trained and calibrated in the use of the criteria increased and was greater than among untrained examiners. Rigid diagnostic criteria are essential in epidemiologic studies. Inter-examiner reliability will be increased if examiners are trained in their use and calibrated in the recognition of clinical signs. Diagnostic criteria should be modified as understanding of the diseases they classify increases. The proposed criteria will enhance the value of studies of HIV-associated periodontal changes and will contribute to that understanding. J Periodontol 1994; 65:236-243.

Key Words: Periodontal diseases/diagnosis; gingivitis/diagnosis; HIV infections/complications; HIV infections/diagnosis.

A number of periodontal changes have been associated with HIV infection, but our knowledge of the epidemiology, microbiology and natural history of these conditions remains limited. ${ }^{1,2}$ One reason for the lack of understanding is that the changes seem to be uncommon..$^{3-8}$ In addition, studies and comparison of results have been hindered by the lack of suitable diagnostic criteria. For example, one group reviewed the oral manifestations of HIV disease, yet their illustrations were of conventional periodontitis rather than HIV-associated periodontitis. ${ }^{9}$ Another group of authors has measured the periodontal health of people with HIV infection using the Community Periodontal Index of Treatment Needs. ${ }^{10}$ This index would not record gingival recession, a sign which may be a distinguishing feature of HIV-periodontitis. ${ }^{11,12}$

\footnotetext{
*University College Dental Hospital, London, UK.

†Oral AIDS Center, Department of Stomatology, University of California, San Francisco, CA.

ःDepartment of Oral Medicine and Pathology, United Medical and Dental Schools, London, UK.
}

This paper describes the development of a set of diagnostic criteria to be used for the identification of HIV-associated periodontal conditions in large-scale epidemiologic studies where many subjects must be examined quickly. The paper also reports on reliability tests to assess the interexaminer reproducibility of diagnoses made using the criteria.

\section{MATERIALS AND METHODS}

Indices of periodontal health are particularly susceptible to inaccuracies for several reasons: 1) there are problems of reliability even with "objective" markers of health, such as probing attachment level; ;3-15 2) some of the markers are subjective; and 3) some periodontal diseases leave irreversible - changes which create problems distinguishing between current and previous disease.

\section{Development of the Criteria}

A set of diagnostic criteria for HIV-associated periodontal conditions were devised based on a literature review and on the clinical experience of the authors (Table 1). The 
The following diagnostic criteria are for use in the diagnosis of periodontal conditions in large scale epidemiologic studies, although it might be possible to amend them to be used in diagnosis in clinical settings. The criteria should allow diagnosis of several conditions in the same mouth. For example, it should be possible, by studying each quadrant, sextant, or tooth at a time, to diagnose conventional periodontitis and HIV-associated periodontitis in the same subject.

\section{Chronic Marginal Gingivitis (Conventional gingivitis)}

To be defined by the presence of erythema, glazing, or swelling of the free gingival margin. With no recession except at the mid-buccal surfaces and no pocketing greater than $3 \mathrm{~mm}$.

\section{Necrotizing Ulcerative Gingivitis (acute)}

To be defined by the presence of conventional gingivitis and both of the following:

a. gingival ulceration radiating from one or more inter-dental papilla tips

b. inflammation limited to the marginal gingiva

\section{HIV-Associated Gingivitis}

To be defined by the presence of gingival erythema manifested by one of the following:

a. punctate erythema of the attached gingiva, ignoring lesions which are largely limited to the marginal gingiva

b. diffuse erythema of the attached gingiva, ignoring lesions which are largely limited to the marginal gingiva

One possible way to recognize such lesions would be to assess

whether the muco-gingival junction is ill-defined.

c. a well-defined red band along the free gingival margin which does not bleed in $50 \%$ or more sites on probing.

Although lacking published evidence, we consider a key feature of the linear HIV-G to be a lack of bleeding on probing.

\section{Ulcerative HIV-Associated Gingivitis}

To be defined by the presence of HIV-associated gingivitis and one of the following:
a. ulceration of the free gingiva
b. spontaneous bleeding

It is assumed that spontaneous bleeding is a sign of micro-ulceration.

\section{Chronic Adult Periodontitis}

To be defined by the presence of gingivitis and attachment loss manifested by one of the following:

a. recession exposing the cemento-enamel junction (excluding the mid-buccal surfaces)

b. pocketing $\geq 4 \mathrm{~mm}$ in two or more sites, excluding pseudo-pockets, third molars, and teeth undergoing orthodontic treatment

Recession present only at the mid-buccal point of the tooth may be associated with toothbrush abrasion and is ignored.

\section{Ulcerative HIV-Associated Periodontitis}

To be defined by the presence of HIV-associated gingivitis and attachment loss where the recession is $\geq 2 \times$ the probing depth* (excluding the mid-buccal surfaces) and one of the following:

a. presence of exposed bone

b. ulceration/necrosis of the attached gingiva

c. patient complaint of severe, "deep," or "bone" pain*

\section{Non-Ulcerative HIV-Associated Periodontitis}

To be defined by the presence of HIV-associated gingivitis and attachment loss where the recession is $\geq 2 \times$ the probing depth (excluding the mid-buccal surfaces) in one of two forms:

a. reverse architecture; i.e., a depression in place of an inter-dental papilla

b. cratering; i.e., a deepening of the inter-dental col where the buccal and palatal/lingual gingivae remain

\section{Necrotizing Periodontitis}

To be defined by the presence of ulceration/necrosis of the attached gingiva and mucosa and/or bone $10 \mathrm{~mm}$ from the cemento-enamel junction or beyond the muco-gingival junction, whichever is nearer.

Although this definition is arbitrary, it is objective. The term necrotizing periodontitis is used to distinguish this type of lesion from necrotizing stomatitis lesions not originating in the periodontium.

Attachment loss can be recorded in two forms, with or without gingivitis. The presence of attachment loss in the absence of gingivitis is not intended to imply active disease but is included to allow collection of data on as many situations as possible. Generalized attachment loss with marginal gingivitis will be assumed to be chronic adult periodontitis (See item 5 above).

9. Attachment Loss With Reverse Architecture or Inter-Dental Cratering

To be defined by the presence of attachment loss $\geq 4 \mathrm{~mm}$ and where the recession is $\geq 2 \times$ the probing depth (excluding the mid-buccal surfaces) in one of two forms:

a. reverse architecture; i.e., a depression in place of an inter-dental papilla

b. cratering; i.e., a deepening of the inter-dental col where the buccal and palatal/lingual gingivae remain

\section{Generalized Attachment Loss}

To be defined by the presence of attachment loss $\geq 4 \mathrm{~mm}$ (excluding the mid-buccal surfaces) without reverse architecture and or cratering.

*Recession must be equal to or greater than twice the probing depth since tissue destruction rather than increased probing depth is a key feature of these conditions. ${ }^{12}$ In practice this means that a lesion with $6 \mathrm{~mm}$ of attachment loss can only have $2 \mathrm{~mm}$ of pocketing to qualify as either form of HIV-P.

criteria reflect descriptions of HIV-associated periodontal conditions in the literature which have been modified to increase objectivity. Their validity was assessed by comparison with clinical photographs and by examining patients with HIV. The criteria were evaluated and refined for ease of use and objectivity in informal tests by the London HIV and Dentistry Group.

These diagnostic criteria distinguish up to 8 conditions which may be found in people infected with HIV. Accuracy was sacrificed in order to simplify the criteria by ignoring uncommon diseases and by assuming that gingivitis in the presence of attachment loss is periodontitis. This kind of assumption is used in epidemiological research, but makes the criteria unsuitable for diagnosis and treatment in individual patients. ${ }^{16}$

The criteria are arranged in a series of tests of increasing stringency and can be arranged in a flow chart. The tests shown in Figure 1 are abbreviated versions of the criteria. 


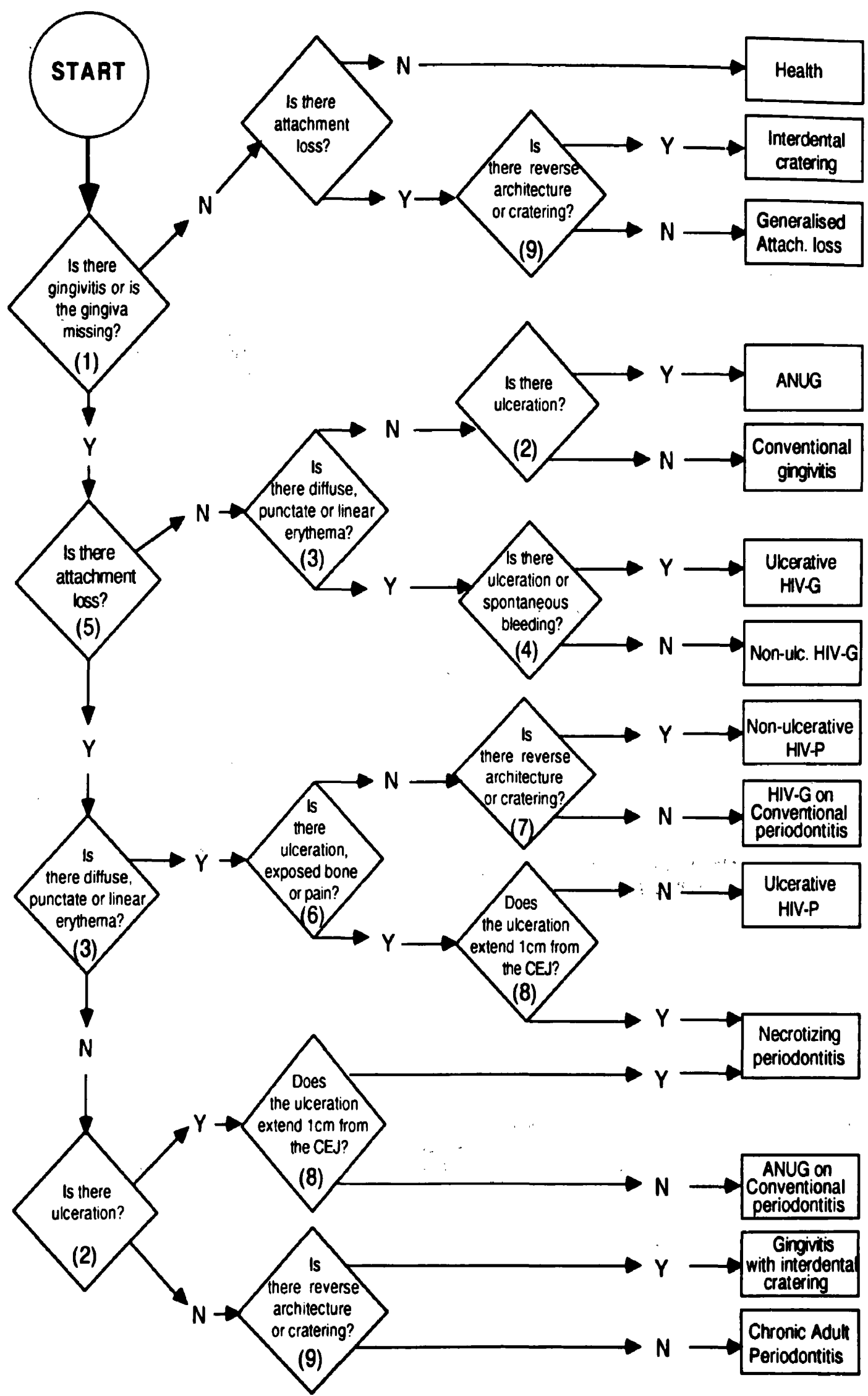

Figure 1. Decision-making flow chart for diagnosis of periodontal diseases. Numbers in pärentheses refer to conditions listed in Table 1.

Since one site may manifest signs of several conditions, some of the criteria may be combined. For example, it should be possible to diagnose HIV-associated changes superimposed on sites with conventional periodontitis.
Assessment of the Reliability of the Criteria

The reliability of the criteria were assessed using a formal experimental design involving pre- and post-testing of subjects randomly assigned to study and control groups. The 
examiners were 9 dentists ( 5 oral medicine specialists and 4 periodontists) working in a university stomatology department. Each had worked in the department for more than one year, was familiar with the literature on HIV-associated periodontal conditions, and had provided care for patients at risk of them.

Initially (Stage 1), all the examiners were shown a series of 20 clinical photographs in the form of color transparencies projected onto a white screen in a darkened room. The photographs were accompanied by brief vignettes containing the age of the patient, all of whom were men, a brief clinical history, and clinical information which could not be elicited from a photograph (e.g., probing depths and bleeding on probing). Each examiner was asked to identify the clinical signs evident in a particular area of the photograph by answering a series of 11 closed questions or by providing alternative answers. Examiners recorded a diagnosis for each photograph by checking one of 14 possible diagnoses or by providing an alternative diagnosis.

The Stage 1 data were sorted by the diagnosis made and the conditions grouped by the strength of association with gingival ulceration. Chi square analyses were used to compare the signs associated with each diagnosis within each group and to identify differences between the ulcerative and non-ulcerative forms of HIV-associated periodontitis (HIVP). The level of agreement (i.e., inter-examiner reliability) among the examiners in the identification of clinical signs was calculated using the kappa statistic for dichotomous variables.

After Stage 1, 5 of the examiners ( 3 oral medicine specialists and 2 periodontists) were selected randomly from the 9 examiners to form a study group. The remaining 4 became the control group. Each member of the study group received a written copy of the criteria to study. The group met one afternoon for training, which consisted of instruction in the use of the criteria, discussion of their validity, simplicity and objectivity, and calibration by comparing diagnoses for a different set of clinical photographs.

One week later (Stage 2), all the examiners were shown the original set of photographs in a different order, again asked to identify the signs and to record diagnoses.

Diagnostic agreement was calculated by determining the proportion of examiners in each group who agreed on each photograph in the Stage 1 and Stage 2 reviews. Changes in the agreement among the study and control groups, and the differences between the groups, were then compared by using the Wilcoxon rank sum statistic.

\section{RESULTS}

\section{Signs Most Commonly Associated With Each Diagnosis}

Table 2 shows the frequency of diagnoses for each of the 20 slides in the Stage 1 review. Table 3 reports the frequency with which the examiners associated clinical signs with each of the diagnoses.
Chronic marginal gingivitis (CMG), HIV-associated gingivitis (HIV-G) without ulceration, and HIV-P without ulceration were rarely associated with ulceration by the examiners. CMG and HIV-G without ulceration were generally associated with a similar set of clinical signs, whereas HIV$P$ without ulceration was associated with a different range of signs. Chi square tests of these data indicate the significant differences in the signs associated with these conditions to be as follows:

1. HIV-P without ulceration was often associated with marginal gingiva of normal coloration, whereas $\mathrm{CMG}$ and HIV-G without ulceration were not.

2. Punctate erythema was associated with HIV-G without ulceration more often than with CMG.

3. A distinct red band of the marginal gingiva was more often associated with CMG than with HIV-G without ulceration or HIV-P without ulceration.

4. Gingival swelling was more often associated with $\mathrm{CMG}$ than with HIV-P without ulceration.

5. Recession was more often associated with HIV-P without ulceration than with $\mathrm{CMG}$ or HIV-G without ulceration.

ANUG, HIV-G with ulceration, HIV-P with ulceration, and necrotizing stomatitis were all associated with swelling and ulceration of the attached gingiva. Chi square analyses revealed the following differences between these conditions: 1) both necrotizing stomatitis and HIV-P with ulceration were associated with recession more than was HIV$\mathrm{G}$ with ulceration. Necrotizing stomatitis was more often associated with recession than was ANUG; 2) necrotizing stomatitis and HIV-P with ulceration were both associated with ulceration of the attached gingiva more than were HIV$\mathrm{G}$ with ulceration or ANUG.

Very different signs were associated with the two forms of HIV-P. As well as lacking ulceration of the marginal or attached gingiva, Chi square analyses indicated that HIV$P$ without ulceration was less often associated with a red band of the marginal gingiva, swelling, or spontaneous bleeding than was HIV-P with ulceration.

\section{Reliability of Recognition of Clinical-Signs}

The kappa statistic is used to measure agreement for dichotomous variables. Kappa scores range between -1 and +1 with a score of zero representing only random agreement. Scores below 0.4 represent poor agreement and above 0.7 represent good agreement. Between these scores agreement is said to be fair/good.

Table 4 presents the kappa scores for the agreement on identification of clinical signs for the study and control groups at both stages of data collection. Some variables for which several choices were offered to examiners (for instance, the data for fiery red, red, normal, or other in the appearance of the marginal gingiva) have been condensed into one sign.

Generally the reliability in all groups for all variables was fair. In Stage 1 the study group showed greater agree- 
Table 2. Frequency of Diagnoses for Each Slide (Stage 1)

\begin{tabular}{|c|c|c|c|c|c|c|c|c|c|c|c|c|c|c|c|}
\hline Slide & Health & $\begin{array}{c}\text { No } \\
\text { Gingivitis } \\
\text { Interdental } \\
\text { Attach- } \\
\text { ment } \\
\text { Loss }\end{array}$ & $\begin{array}{c}\text { No } \\
\text { Gingivitis } \\
\text { General- } \\
\text { ized } \\
\text { Attach- } \\
\text { ment } \\
\text { Loss } \\
\end{array}$ & $\begin{array}{c}\text { Chronic } \\
\text { Marginal } \\
\text { Gingi- } \\
\text { vitis } \\
\end{array}$ & ANUG & $\begin{array}{l}\text { Periodon- } \\
\text { titis } \\
\text { Localized } \\
\text { Attach- } \\
\text { ment } \\
\text { Loss } \\
\end{array}$ & $\begin{array}{l}\text { Periodon- } \\
\text { titis } \\
\text { General- } \\
\text { ized } \\
\text { Attach- } \\
\text { ment } \\
\text { Loss } \\
\end{array}$ & $\begin{array}{l}\text { HIV-G } \\
\text { Without } \\
\text { Ulcera- } \\
\text { tion } \\
\end{array}$ & $\begin{array}{l}\text { HIV-G } \\
\text { With } \\
\text { Uicera- } \\
\text { tion }\end{array}$ & $\begin{array}{c}\text { HIV-P } \\
\text { Without } \\
\text { Ulcera- } \\
\text { tion }\end{array}$ & $\begin{array}{l}\text { HIV-P } \\
\text { With } \\
\text { Ulcera- } \\
\text { tion }\end{array}$ & $\begin{array}{l}\text { Necrotiz- } \\
\text { ing } \\
\text { Periodon- } \\
\text { titis }\end{array}$ & $\begin{array}{l}\text { HIV-G No } \\
\text { Ulceration } \\
\text { on } \\
\text { - Periodon- } \\
\text { titis } \\
\end{array}$ & $\begin{array}{l}\text { ANUG } \\
\text { on } \\
\text { Periodon- } \\
\text { titis }\end{array}$ & Other \\
\hline 1 & & & & 1 & 3 & & & & & & 3 & 2 & & & \\
\hline 2 & & & & 2 & 1 & 1 & & 2 & 3 & & & & & & \\
\hline 3 & & & & & & & & & & & 4 & 4 & & & 1 \\
\hline 4 & & & & 2 & 1 & 1 & 2 & & & & & 2 & & & 3 \\
\hline 5 & & & & & & 4 & 1 & & & 1 & 2 & 1 & & & 1 \\
\hline 6 & & & & 2 & 1 & 2 & & & 2 & 2 & & & & & \\
\hline 7 & & & & 1 & & 5 & 2 & & & & & & & & \\
\hline 8 & & & & & & 1 & & 5 & & & & & & & 1 \\
\hline 9 & 1 & & 6 & & & & 2 & & & & & & & & \\
\hline 10 & & & & & 4 & & & 1 & 3 & & & 1 & & & \\
\hline 11 & & & & & 1 & & 3 & & & & 1 & 1 & 1 & & 2 \\
\hline 12 & & & & & 1 & 3 & & & & 1 & & 1 & & 2 & 2 \\
\hline 13 & & & & & 1 & & & & & & 3 & 2 & 1 & 2 & \\
\hline 14 & & & & & 4 & & & & 1 & & 2 & 2 & & & \\
\hline 15 & & & & & & 3 & & & & 3 & 1 & 2 & & & \\
\hline 16 & & & & 5 & & & & 3 & & & & & & & \\
\hline 17 & & & & & 6 & & & 1 & 2 & & & & & & 1 \\
\hline 18 & & & & & 1 & 2 & 1 & & & 3 & 1 & & & & \\
\hline 19 & & & & 4 & 1 & & & 4 & & & & & & & \\
\hline 20 & & & & & 1 & & & 2 & 2 & & 3 & & & 1 & \\
\hline
\end{tabular}

ment than the control group in the recognition of increased probing depth. In Stage 2 the calibrated group showed greater reliability than the control group for two variables (color of marginal gingiva and probing depth).

\section{Reliability of Diagnosis}

The proportion of subjects agreeing on the diagnosis for each slide in the Stage 1 and Stage 2 reviews was tabulated for the study and control groups. The differences in the number of reviewers agreeing before and after the calibration session were determined and analyzed by the Wilcoxon rank-sum test. The median change in agreement level between the two reviews was 0.0 in the control group and 0.3 in the study group $(P=0.0113)$, indicating that the training had improved the level of agreement.

The proportion of subjects in the study and control groups

Table 3. Signs Associated With Each Diagnosis (Stage 1)

\begin{tabular}{|c|c|c|c|c|c|c|c|c|c|c|c|c|}
\hline \multirow[b]{2}{*}{ Diagnosis (n) } & \multicolumn{3}{|c|}{ Marginal Gingiva } & \multicolumn{3}{|c|}{ Attached Gingiva } & \multirow{2}{*}{$\begin{array}{l}\text { Red } \\
\text { Band } \\
\mathrm{n}(\%) \\
\end{array}$} & \multirow[b]{2}{*}{$\begin{array}{c}\text { Swelling } \\
\mathrm{n}(\%)\end{array}$} & \multirow{2}{*}{$\begin{array}{l}\text { Spontaneous } \\
\text { bleeding } \\
\mathrm{n}(\%)\end{array}$} & \multirow{2}{*}{$\begin{array}{c}\text { Recession } \\
\mathrm{n}(\%)\end{array}$} & \multirow{2}{*}{$\begin{array}{c}\text { Ulcerative } \\
\text { Marginal } \\
\text { Gingiva } \\
\text { n(\%) }\end{array}$} & \multirow{2}{*}{$\begin{array}{c}\text { Ulcerative } \\
\text { Attached } \\
\text { Gingiva } \\
\mathbf{n}(\%) \\
\end{array}$} \\
\hline & $\begin{array}{c}\text { Fiery Red } \\
\mathrm{n}(\%)\end{array}$ & $\begin{array}{l}\text { Red } \\
\mathrm{n}(\%)\end{array}$ & $\begin{array}{l}\text { Normal } \\
\mathrm{n}(\%)\end{array}$ & $\begin{array}{c}\text { Diff. red } \\
\mathrm{n}(\%)\end{array}$ & $\begin{array}{l}\text { Punct. red } \\
\mathrm{n}(\%)\end{array}$ & $\begin{array}{c}\text { Normal } \\
\mathrm{n}(\%)\end{array}$ & & & & & & \\
\hline CMG (17) & $5(30)$ & $11(65)^{*}$ & $0(0)^{*}$ & $8(47)$ & $2(12)^{*}$ & $6(35)$ & $14(82)^{*+}$ & $17(100)^{*}$ & $0(0)$ & $5(29)^{\dagger}$ & $4(24)$ & $1(6)$ \\
\hline HIVG-Ulc (18) & $7(39)$ & $10(56)^{\dagger}$ & $0(0)^{\dagger}$ & $10(56)$ & $5(28)^{*}$ & $2(11)$ & $9(50)^{*}$ & $16(89)$ & $3(17)$ & $3(17)^{*}$ & $0(0)$ & $0(0)$ \\
\hline HIVP-Ulc (10) & $1(10)$ & $2(20)^{* \dagger}$ & $9(90)^{*+\neq}$ & $3(30)$ & $2(20)$ & $6(60)^{*}$ & $2(20)^{\ddagger \ddagger}$ & $7(70)^{* \dagger}$ & $1(10)^{*}$ & $9(90)^{\dagger *}$ & $1(10)^{*}$ & $0(0)^{*}$ \\
\hline ANUG (26) & $9(35)$ & $16(62)$ & $2(8)$ & $15(58)$ & $6(23)$ & $6(23)$ & $11(42)$ & $22(85)$ & $11(42)$ & $16(62)^{\ddagger}$ & $24(92)$ & $3(12)^{t t}$ \\
\hline $\begin{array}{l}\text { HIVG + Ulc } \\
\text { (13) }\end{array}$ & $6(46)$ & $4(31)$ & $1(8)$ & $8(62)$ & $2(15)$ & $2(15)$ & $5(38)$ & $13(100)$ & $5(38)$ & $4(31)^{84}$ & $13(100)$ & $2(15)^{8 *}$ \\
\hline $\mathrm{HIVP}+\mathrm{Ulc}(20)$ & $9(45)$ & $8(40)$ & $0(0)^{\ddagger}$ & $9(45)$ & $7(35)$ & $1(5)^{*}$ & $11(55)^{\ddagger}$ & $17(85)^{\dagger}$ & $9(45)^{*}$ & $15(75)^{\Upsilon}$ & $19(95)^{*}$ & $14(70)^{* * *}$ \\
\hline Nec perio (18) & $6(33)$ & $9(50)$ & $1(6)$ & $9(50)$ & $7(39)$ & $1(6)$ & $7(39)$ & $14(78)$ & $12(67)$ & $18(100)^{5 t}$ & $13(72)$ & $10(56)^{5 \dagger}$ \\
\hline
\end{tabular}

*, ${ }^{\dagger}, \neq$, \&, ", and * all denote $P<0.05$ for differences within each column by chi square analysis.

Pocketing, mucosal ulceration, and exposure of bone were associated similarly with all the diagnoses. 
Table 4. Kappa Scores for Inter-Rater Agreement on Signs of Periodontal Diseases

\begin{tabular}{|c|c|c|c|c|}
\hline \multirow[b]{2}{*}{ Sign } & \multicolumn{2}{|c|}{ Stage 1} & \multicolumn{2}{|c|}{ Stage 2} \\
\hline & Study (95\% CI) & Control $(95 \% \mathrm{CI})$ & Study (95\% CI) & Control $(95 \% \mathrm{CI})$ \\
\hline $\begin{array}{l}\overline{\text { Red }} \\
\text { marginal } \\
\text { gingiva }\end{array}$ & $0.39(0.24,0.53)$ & $0.64(0.46,0.82)$ & $0.57(0.42,0.72)^{\dagger}$ & $0.01(-0.17,0.19)^{\dagger}$ \\
\hline $\begin{array}{l}\text { Red } \\
\text { attached } \\
\text { gingiva }\end{array}$ & $0.48(0.33,0.63)$ & $0.50(0.33,0.69)$ & $0.53(0.38,0.68)$ & $0.46(0.28,0.64)$ \\
\hline $\begin{array}{l}\text { Red } \\
\text { band }\end{array}$ & $0.45(0.31,0.63)$ & $0.38(0.19,0.56)$ & $0.59(0.45,0.74)$ & $0.44(0.27,0.62)$ \\
\hline Swelling & $0.31(0.17,0.44)$ & $0.21(0.03,0.39)$ & $0.17(0.03,0.31)$ & $0.19(0.01,0.37)$ \\
\hline $\begin{array}{l}\text { Spontaneous } \\
\text { bleeding }\end{array}$ & s $0.33(0.18,0.48)$ & $0.53(0.34,0.72)$ & $0.63(0.44,0.82)$ & $0.30(0.11,0.49)$ \\
\hline $\begin{array}{l}\text { Increased } \\
\text { probing } \\
\text { depth }\end{array}$ & $0.75(0.60,0.89)^{*}$ & $0.31(0.12,0.50)^{*}$ & $1.00(0.84,1.00)^{\ddagger}$ & $0.24(0.06,0.41)^{\ddagger}$ \\
\hline Recession & $0.50(0.34,0.67)$ & $0.30(0.08,0.52)$ & $0.71(0.55,0.88)$ & $0.76(0.54,0.98)$ \\
\hline $\begin{array}{l}\text { Ulceration } \\
\text { marginal } \\
\text { gingiva }\end{array}$ & $0.51(0.37,0.65)$ & $0.71(0.52,0.90)$ & $0.95(0.81,1.00)$ & $0.64(0.46,0.82)$ \\
\hline $\begin{array}{l}\text { Ulceration } \\
\text { attached } \\
\text { gingiva }\end{array}$ & $0.38(0.23,0.62)$ & $0.27(0.07,0.46)$ & $0.57(0.42,0.71)$ & $0.59(0.40,0.82)$ \\
\hline $\begin{array}{l}\text { Ulceration } \\
\text { mucosa }\end{array}$ & $0.48(0.34,0.62)$ & $0.48(0.27,0.69)$ & $0.74(0.59,0.88)$ & $0.64(0.45,0.82)$ \\
\hline $\begin{array}{l}\text { Exposed } \\
\text { bone }\end{array}$ & $0.08(-0.06,0.23)$ & $-0.04(-0.23,0.16)$ & $-0.01(-0.16,0.13)$ & $-0.01(-0.20,0.17)$ \\
\hline
\end{tabular}

${ }^{*},{ }^{\dagger}$, and ${ }^{\ddagger}$ indicate $P<0.05$ using the $95 \%$ confidence intervals.

who agreed on the diagnosis of each slide in the Stage 2 review was also analyzed by the Wilcoxon test. The median agreement for the control group was 0.33 and for the study group, $0.60(P=0.0054)$, indicating a significantly higher level of agreement in the trained group.

\section{DISCUSSION}

In this study, data were collected by inviting examiners to identify signs and record diagnoses from clinical photographs. This technique limits the information available to the examiners and may have reduced their ability to observe and diagnose accurately. However, this is the most appropriate technique for calibration for acute conditions which are both uncommon and painful. Moreover, the use of photographs standardizes the conditions of the clinical examination. The technique is widely used in calibration exercises, and the findings of this study are compatible with our understanding of diagnosis of diseases. Examiner reliability is often poor, but training and calibration improve reliability of diagnosis of periodontal changes. ${ }^{17-19}$

\section{Signs Associated With Diagnoses}

Most examiners associated a similar set of clinical signs with the diagnoses of chronic marginal gingivitis and HIVassociated gingivitis (Table 3 ). The only signs associated with CMG and HIV-G with different frequency were punctate erythema of the attached gingiva (12\% and $28 \%$ of diagnoses, respectively) and a distinct red band of the gingiva ( $82 \%$ and $50 \%$ of the diagnoses, respectively). Thus, with differences between the frequencies of $16 \%$ and $32 \%$, in only a maximum of $48 \%$ of cases can the distinction between these two conditions be attributed to these two variables. This figure may be an over-estimate, since examiners more commonly associated a distinct red band with conventional gingivitis than with HIV-gingivitis. This sign is strongly associated with HIV-gingivitis in the literature, ${ }^{12}$ suggesting that diagnoses were made intuitively rather than using objective criteria.

The signs associated with HIV-P with ulceration were very different from those associated with HIV-P without ulceration. HIV-P without ulceration lacked the red band of the marginal gingiva, swelling, and spontaneous bleeding associated with the ulcerated form. HIV-P without ulceration was distinguished from CMG and HIV-G without ulceration by a normal coloration of the marginal gingiva and lack of gingival swelling. The distinction between HIV$P$ with and without ulceration is new and was introduced to reflect two different presentations in the literature. ${ }^{20}$ While it is possible that the lesions which the examiners termed "HIV-periodontitis without ulceration" represented a destructive disease, another explanation is that such lesions represent areas of arrested localized destruction. In that case, the term "periodontitis" is a misnomer and "localized inter-dental attachment loss" might be a more appropriate 
description. This condition may be arrested or treated HIVperiodontitis but firm conclusions cannot be drawn without longitudinal data.

Examiners associated ANUG, HIV-G with ulceration, HIV-P with ulceration and necrotizing stomatitis with swelling and ulceration of the gingiva. Necrotizing stomatitis and HIV-P with ulceration were associated with more extensive tissue damage (as evidenced by recession in Table 3 ) but there were no qualitative differences in the features associated with the diagnoses. These data suggest that diagnoses were not made by use of criteria common to all examiners, a hypothesis supported by the poor agreement on diagnoses.

Other signs or a particular combination of signs may have led examiners to a particular diagnosis for each photograph. However, the data do not suggest this, and the poor agreement on identification of signs and on diagnoses further suggests that diagnoses were not made using objective criteria. These findings suggest that examiners require rigid criteria to make reproducible diagnoses.

\section{Reliability of Recognizing Signs}

Diagnoses are made by assimilating a pattern of clinical signs to a pattern which corresponds to a known disease. If examiners cannot agree on whether a sign is present or absent they will be unable to agree on diagnoses. In both stages of this study, examiners showed only fair reliability identifying straightforward clinical signs such as redness of the marginal gingiva or even ulceration (Table 4). This emphasizes the need for careful calibration before any study involving several examiners.

After calibration, the study group agreed on the color of the marginal gingiva more than the controls. This improvement is encouraging, but constitutes only one variable of 11 studied. In a previous study on the agreement of scoring of gingival indices, Alexander et al. did not detect any improvement in agreement until there had been 3 calibration sessions. ${ }^{18}$ Our calibration involved only reading of the criteria and a single 2-hour session with clinical photographs; thus, profound improvements were not expected.

\section{Reliability of Diagnosis}

Given the intuitive nature of the diagnoses and the mediocre level of agreement on the clinical signs, it is not surprising that agreement on diagnoses was poor in the initial stage of this study (Table 2). The implications of this are important. Authors who have reported on HIV-associated periodontal conditions might not have been looking at this range of conditions at all. It must be stressed that the examiners in this study were experts who were experienced and well informed. All worked in the same department of a dental school and it is likely that there is greater agreement among this group than among less experienced examiners not working in such close proximity.

The data in this study reveal significant improvement in the inter-examiner reliability of examiners who were trained and calibrated in the use of the diagnostic criteria, with no corresponding increase in agreement among a non-calibrated control group. This resulted in significantly more agreement among the calibrated group. These findings confirm the value of these techniques and demonstrate the reliability of the criteria.

\section{Validity of the Criteria}

The criteria were based on the clinical experience of the authors and on a review of the literature. Since the authors are based only in London and San Francisco, it is difficult to ascertain whether similar manifestations occur elsewhere. Truly valid criteria can only be decided upon at a consensus conference with many centers represented, but previous conferences have failed to produce workable, mutually exclusive, and objective criteria. ${ }^{21,22}$ Given the conflicts in reports of HIV-associated periodontal changes and the vagueness of the literature, the proposed criteria must remain an interim measure. One way to gain accurate information on HIV-associated periodontal changes is to record clinical parameters in large numbers of representative samples of people with HIV infection. ${ }^{23}$ If signs such as gingival redness, bleeding on probing, ulceration, and necrosis are recorded, the validity of these or other criteria could be tested by applying them on a post hoc basis. Until then it is uncertain whether this or any other set of criteria is valid.

Such studies are under way, but will take some time to complete. If the periodontal changes associated with HIV are uncommon, several similar studies may be required until the full picture emerges. In the meantime, some form of simple diagnostic criteria that can be quickly and inexpensively applied are required for large scale epidemiologic studies, where comprehensive periodontal measurements are impractical. The set of criteria used in this study meets that need. The value of objectively defined criteria is that the findings of studies using them will have common meaning and can be compared with less uncertainty.

The criteria can be tested in the same way. If repeated, large scale, controlled studies do not detect differences in the prevalence of certain conditions in people with and without HIV, then either the criteria are invalid or the conditions do not exist. That is to say, the criteria must have high positive predictive values for HIV infection.

\section{Simplicity of the Criteria}

The flow chart (Fig. 1) was designed to formalize the decisions used to make periodontal diagnoses. It uses information which should already be collected during a clinical examination and which should be incorporated into any diagnostic process. Previous research has employed criteria which were as comprehensive as those used in this investigation, but which were not mutually exclusive. ${ }^{8}$

One way of simplifying this diagnostic system would be to use only the written criteria. This would make the system easier to use by reducing the range of possible diagnoses, but simplification would be achieved at the expense of pre- 
cision. Investigators should decide on this compromise within the context of each study.

The flow chart need not be memorized, but should be studied carefully as part of the training process. Training is regarded as an essential component of any periodontal study, ${ }^{17,19}$ but has only been reported in 3 studies of HIVassociated periodontal diseases. ${ }^{7,8,23}$ Examiners may be resistant to being trained and calibrated in the use of particular criteria, but the consequences of this resistance may be reflected in our present poor understanding of HIV-associated periodontal diseases. This study has demonstrated that the techniques are effective for this range of conditions.

\section{Conclusion}

A group of experienced examiners showed only fair reliability in the recognition of clinical signs of periodontal conditions. They made diagnoses intuitively and had only fair agreement on the diagnosis of periodontal conditions. The inter-examiner reliability of examiners trained and calibrated in the use of a set of diagnostic criteria increased and was greater than among untrained examiners.

Rigid diagnostic criteria are an important feature of any epidemiologic study. Inter-examiner reliability will be increased if examiners are trained in their use and calibrated in the recognition of clinical signs. These measures will increase the value of studies of HIV-associated periodontal changes and will contribute to our understanding of them.

\section{Acknowledgments}

The authors are grateful for the assistance of -Drs. Gary Armitage, Michael Danford, Caroline Dodd, Deborah Greenspan, Gad Heinic, Mitchell Katz, Shepherd Levine, Laurie MacPhail, and David Richards and members of the London HIV and Dentistry Group. This research was funded through the National Institute for Dental Research program project grant P01-DE07946. Peter Robinson is the SmithKline Beecham Research Fellow of the Oral and Dental Research Trust and was partly funded for this project by a Colgate Research Award.

\section{REFERENCES}

1. Winkler JR, Murray PA. A potential intra-oral expression of AIDS may be rapidly progressive periodontitis. J Calif Dent Assoc 1987; 15:20-24.

2. Robinson PG. Periodontal diseases and HIV infection: A review of the literature. J Clin Periodontol 1992; 19:609-614.

3. Klein RS, Quart AM, Butkus Small C. Periodontal disease in heterosexuals with acquired immunodeficiency syndrome. $J$ Periodontol 1991; 62:535-540.

4. Cornick DER, Robinson PG. A three year study of the periodontal condition in HIV positive men. J Dent Res 1991; 70:688.

5. Drinkard CR, Decher L, Little JW, et al. Periodontal status of indi- viduals in early stages of human immunodeficiency virus infection. Comm Dent Oral Epidemiol 1991; 19:281-285.

6. Friedman RB, Gunsolley J, Gentry A, Dinius A, Kaplowitz L, Settle J. Periodontal status of HIV-seropositive and AIDS patients. $J$ Periodontol 1991; 62:623-627.

7. Melnick SL, Engel D, Truelove E, et al. Oral mucosal lesions: Association with the presence of antibodies to the human immunodeficiency virus. Oral Surg Oral Med Oral Pathol 1989; 68:37-43.

8. Riley C, London JP, Burmeister JA. Periodontal health in 200 HIVpositive patients. J Oral Pathol Med 1991; 21:124-127.

9. Scully C, Epstein JB, Porter S, Luker L. Recognition of oral lesions of HIV infection. 3. Periodontal disease and less common lesions. $\mathrm{Br}$ Dent $J$ 1990; 169:370-372.

10. Lucht E, Heimdahl A, Nord CE. Periodontal disease in HIV-infected patients in relation to lymphocyte subsets and specific micro-organisms. J Clin Periodontol 1991; 18:252-256.

11. Ainamo J, Barmes D, Beagrie G, Cutress T, Martin J, Sardo-Infirri J. Development of the World Health Organization (WHO) Community Periodontal Index of Treatment Needs (CPITN). Int Dent J 1982; 32:281-291.

12. Winkler JR, Murray PA, Grassi M, Hammerle C. Diagnosis and management of HIV-associated periodontal lesions. J Am Dent Assoc 1989; 119(Suppl.):25S-34S.

13. Imrey PB. Considerations in the statistical analysis of clinical trials in periodontitis. J Clin Periodontol 1986; 13:517-528.

14. Espeland MA, Zappa UE, Hogan PE, Simona C, Graf H. Crosssectional and longitudinal reliability for clinical measurement of attachment loss. J Clin Periodontol 1991; 18:126-133.

15. Sterne JAC, Curtiss MA, Gillett IR, et al. Statistical models for data from periodontal research. J Clin Periodontol 1990; 17:129-137.

16. Tan EM, Cohen AS, Fries JF, et al. The 1982 revised criteria for the classification of systemic lupus erythematosus. Arthritis Rheum 1982; 25:1271-1277.

17. Cowell CR, Saxton CA, Sheiham A, Wagg BJ. Testing therapeutic measures for controlling chronic gingivitis in man: A suggested protocol. J Clin Periodontol 1975; 2:231-240.

18. Alexander AG, Leon AR, Ribbons JW, Morganstein SI. An assessment of the inter- and intra-examiner agreement in scoring gingivitis clinically. J Periodont Res 1971; 6:146-151.

19. Smith LW, Suomi JD, Greene JC, Barbano JP. A study of intraexaminer variation in scoring oral hygiene status, gingival inflammation and epithelial attachment level. J Periodontol 1970; 41:671674.

20. Greenspan D, Greenspan JS, Pindborg JJ, Schiodt M. AIDS and the Dental Team. Copenhagen: Munksgaard; 1986:43.

21. Greenspan JS, Barr CE, Sciubba JJ, Winkler JW, and the US Oral AIDS Collaborative Group. Oral manifestations of HIV infection. Oral Surg Oral Med Oral Pathol 1992; 73:142-144.

22. EEC Clearinghouse on Oral Problems Related to HIV Infection and WHO Collaborating Centre on Oral Manifestations of the Human Immunodeficiency Virus. An update of the classification and diagnostic criteria of oral lesions and HIV infection. J Oral Pathol Med 1991; 20:97-100.

23. Swango PA, Kleinman DV, Konzelman JL. HIV and periodontal health. A study of military personnel with HIV. $J$ Am Dent Assoc $1991 ; 122: 49-54$.

Send reprint requests to: Dr. Peter Robinson, Surgery V, University College Dental Hospital, Mortimer Market, London WC1E 6AU, UK. Accepted for publication September 13, 1993. 


\title{
Periodontal diseases and HIV
} infection

\author{
Peter Robinson \\ University College Dental Hospital, London, \\ UK
}

\section{A review of the literature}

\begin{abstract}
Robinson P: Periodontal diseases and HIV infection A review of the literature. J Clin Periodontol 1992; 19: 609-614.
\end{abstract}

Abstract. There have been many references in the literature to HIV-related periodontal diseases, which although poorly substantiated, seem to have established them as part of the expected range of HIV-associated conditions. The original studies have produced conflicting reports which may stem from shortcomings in design. Consequently, the picture remains confused with respect to the classification, epidemiology, microbiology, natural history and management of HIVrelated periodontal diseases. Future studies should give greater attention to sampling methods, the use of control groups and defining criteria. This will allow comparison of data between centres and facilitate study of what may be an uncommon disease.
Key words: HIV; periodontal disease.

Accepted for publication 29 August 1991
Periodontal diseases have long been associated with systemic diseases (Emslie 1963, Sznajder et al. i978). Getico \& Slots (1984) have reviewed the periodontal changes directly associated with defects in the immune system. particularly those described in patients with neutrophil and lymphocyte disorders. HIV infection results in a depletion of $\mathrm{T}$ helper lymphocytes and impairment of neutrophil function, therefore changes in the periodonteum might be expected as part of this disease.

There are numerous references to HIV-related periodontal diseases in the literature. Although some of these are poorly substantiated, they have established that periodontal diseases,are part of the expected range of HIV associated conditions (Anon 1988, Dennison et al. 1985. Farthing 1986, Farthing et al. 1988. Greenspan et al. 1986. Pindborg 1988. Sadoun 1987. Schulten et al. 1990, Silverman et al. 1986. EEC 1991). However. the original reports of the disease consist of very few cases. Few reports describe the methods used to diagnose or monitor the condition. Some are case reports and a few have included details of case management (Abel \& Andriolo 1989. Pindborg 1986, Pindborg \& Holmstrupp 1987. Rosenstein et al. 1989). The larger studies have produced conflicting reports on the character- istics. epidemiology, microbiology and natural history of the diseases.

This paper reviews the literature relating to HIV-associated periodontal diseases and pays particular attention to their classification, epidemiology microbiology, natural history and management.

\section{Classification of HIV-associated periodontal diseases}

One group has carried out more thoroughly documented research of HIVrelated periodontal diseases; the Oral AIDS Centre of the University of California at San Francisco (UCSF). In 1986 they described two periodontal lesions. a generalised atypical gingivitis (ATYP) and an ANUG-like lesion (Winkler et al. 1986). The gingivitis was characterised by bright erythema. often extending to the alveolar mucosa. with oedema. and usually affecting all the teeth but not responding to improved oral hygiene. More recently they have renamed this condition HIV-gingivitis (HIV-G) (Winkler et al. 1988a) and described three different types of erythema: (1) and intense red linear border at the gingival margin: (2) petechiae like red patches affecting the attached and/ or unattached gingiva: (3) a generalised erythema affecting the attached and/or unattached gingiva.

The ANUG.like lesion was originally named AIDS virus associated periodontitis (AVAP) and subsequently HIVperiodontitis (HIV-P). It is characterised by severe pain. marked localised soft tissue necrosis, ulceration and interproximal cratering. The lesions have not been associated with deep pocket formation but a loss of crestal bone coincident with soft tissue destruction has been described. Bone may be exposed and subsequently sequestrate (Winkler et al. 1988a). Smith et al. (1991) noted this occurrence in 10 patients in a study of 103 people with HIV (47 injecting drug users and 53 homosexual men). It should be noted that this figure suggests a higher incidence than many of the other studies on the epidemiology of this range of diseases. The same group of workers have also recorded a case of HIV-P where the bone loss was sufficient to cause an oro-antral fistula (Felix et al. 1991).

These findings are not entirely consistent with those reported at other centres. Pekovic et al. (1988) and Gornitsky \& Pekovic (1987) have described rapid periodontal pocket formation in people with HIV and AIDS. Rosenstein et al. (1989) also noted horizontal rapid bone loss with only moderate inflam- 
mation in one case. Winkler et al. (1988a) suggested that geographical differences may explain the variation in these findings. He commented that the UCSF study groups had very low levels of periodontal disease at baseline. This may allow the detection of subtle changes in periodontal health which might otherwise be masked by conventional periodontal disease. There is no direct evidence for either of these theories and the scarcity of data prevents any firm conclusions being drawn.

In the absence of a universally accepted classification of HIV-associated periodontal diseases with appropriate defining criteria. the classification employed at UCSF will be used in the remainder of this article.

\section{Possible association between HIV infection and ANUG}

Several systemic diseases have been associated with ANUG including blood dyscrasias. malnutrition. acute herpetic gingivo-stomatitis and measles (Emslie 1963, Cogen 1990).

Dennison et al. (1985) described acute necrotising ulcerative gingivitis in a group of homosexual men not tested for HIV infection. Several case reports of HIV-associated ANUG exist (Pindborg 1986. Pindborg \& Holmstrupp 1987) and the disease has been included in some lists of the oral manifestations of HIV infection (Greenspan et al. 1986. Pindborg 1988).

Genco (1990) has compared the necrotizing ulcerative periodontitis which results from recurrent episodes of ANUG with the periodontal changes seen in HIV infection. Similarly, there are two reports of people with AIDS with 'gangrenous stomatitis' (cancrum oris or noma) (Akula et al. 1989, Giovanni et al. 1989). Cancrum Oris is an extension of ANUG (Emslie 1963). and Winkler et al. (1989b) have suggested that such lesions may originate in the periodontium of people with HIV infection.

Winkler \& Murray (1987) have distinguished HIV-P from ANUG by its rapid progression. In one patient $90 \%$ attachment loss was documented over a six month period. ANUG only results in loss of bone after multiple episodes over several years. More recently, there have been case reports of HIV-P complicated by destruction of alveolar bone (San Giacomo et al. 1990, Williams et al. 1990. Felix et al. 1991). San Gianco- mo and colleagues imply this 'progressive osseous destruction' is an extension of HIV-P. whereas Williams et al. (1990) describe 'necrotizing stomatitis' as a complication of the HIV-P. Neither group has discussed the differentiation of their observations from Cancrum oris.

Murray et al. (1988) have investigated the subgingival plaque of a small num. ber of sites with HIV-P and found the levels of spirochaetes present in the lesions are variable and not consistent with the microflora associated with ANUG.

\section{Epidemiology}

There is little information on the prevalence of HIV-related periodontal diseases. Some research has been carried out on patients attending for dental treatment. Such use of self-selected samples may over-estimate the prevalence of periodontal diseases because the patients may have been attending the clinic because they had gingival symptoms. Other studies have been poorly controlled. whilst others have not used well defined criteria to diagnose periodontal diseases.

Murray et al. (1991) reported a high prevalence of HIV-G (49\%) and HIV-P (22\%) among a group of injecting drug users in New. Jersey who had sought dental treatment at a special clinic. Likewise. Zakarian et al. (1988) have retrospectively reviewed 150 patients with AIDS or ARC treated at a dedicated clinic in New York. Of these, 12\%' had manifested interproximal necrosis resembling acute necrotizing ulcerative gingivitis and $5 \%$ had presented with rapidly progressive periodontitis.

Zambon et al. (1990) examined 37 patients with AIDS and found clinical signs ther considered consistent with HIV-G in $7(19 \%)$, HIV-P in 1 patient and ANLG in 6 patients. No mention is made of how this sample was selected. Schulten et al. (1990) noted periodontal disease in 9 of 70 patients $(13 \%)(34$ with AIDS and 36 with HIV infection) attending an internal medicine department. No mention was made of the nature or severity of the disease or of the diagnostic criteria used.

Tukutuku et al. (1990) diagnosed 'moderate' or 'severe' gingivitis in 55 $(66 \%)$ of 83 hospitalised patients with AIDS in Zaire. The authors noted an increased prevalence of gingivitis in this sample compared with prevalences detected by other examiners in Nigeria and Zaire in two studies carried out 15 and 11 years earlier (Henshaw \& Adenjubi 1975, Ducka-Karsa 1979). The use of different examiners and the time between the studies calls this comparison into question.

Other workers have found little or no evidence of unusual periodontal diseases in people with HIV infection. For example, in a small study of 15 people with HIV infection attending a hospital in Mexico, only 2 cases corresponding to descriptions of HIV-G were identified. No cases of HIV-P were found (Apaiza et al. 1991).

Phelan et al. (1987) examined a consecutive sample of 103 people $(57 \%$ drug users and $28 \%$ homo- or bisexual men) with AIDS who had not been referred with an oral manifestation of HIV infection. No cases of 'severe periodontal disease' were noted and this report contains no reference to gingival disease other than 1 case of gingival bleeding.

Melnick et al. (1989) carried out a controlled. blinded study of 803 homosexual men (141 with HIV infection) attending a counselling centre in Seattle. Severe periodontal disease was diagnosed by the presence of "extensive gingival recession with marked gingival inflammation, clinically evident alveolar bone loss and extrusion of pus from gingival pockets'. Seven $(5 \%)$ of the HIV positive subjects had severe disease but the prevalence of periodontal disease in the control group was extremely low, with only 1 case in 606 men (mean age 34.4 years). Based on these findings. the authors concluded that periodontal disease had a significant association with HIV infection but was not 'highly common:

Barr et al. (1990, 1991) have presented baseline data on 101 HIV in fected and 34 non-infected homosexual men. No significant differences were found between the periodontal health of the two groups although a significant correlation was identified between plaque index scores and gingival indices for the whole group. At I year follow up (75 men with HIV infection and 27 without). the prevalence of periodontitis $(3 \%)$. ANUG $(2 \%)$ and atypical gingivitis $(3 \%)$ was low in the whole group and no significant differences in prevalence were found between the infected and control groups. However, all the subjects had received 3-monthly scaling and periodontal prophylaxis. and these 
findings may reflect the success of the preventive intervention and cannot be compared with the incidence of periodontal diseases in untreated populations.

Cornick \& Robinson (1991) have followed a group of homosexual men with HIV infection for 3 years. Initially the sample consisted of 57 men with known HIV infection, 8 known not to be infected and 28 of indeterminate status. After three years only 25 men with HIV infection remained in the study. No significant differences in the gingivitis indices, probing depths, attachment levels or crestal bone height were associated with the presence of HIV infection or increasing severity of HIV disease.

The UCSF team has reviewed the diagnosis and management of more than 400 patients with HIV-G and HIV-P over 6 years and published quantitative data for 113 of these (Winkler et al. 1988a. 1988b, 1989a). These figures must be considered in the context of the prevalence of HIV infection which is very high in San Francisco. At the time of these reports, the cumulative total of AIDS cases was 4000 and the prevalence of HIV infection would have been much greater. This suggests the incidence of HIV related periodontal diseases is not high. Winkler et al. (1990) conducted a controlled, blind study of 18 San Francisco men with asymptomatic HIV infection and found no cases of HIV-G or HIV-P nor any significant difference in their periodontal health. It is possible that the sample was too small to detect what may be an uncommon disease.

The disparity in the findings of all of these studies may result from the lack of universally applied diagnostic criteria. the use of self-selected samples, lack of controls and the study of subjects receiving preventive periodontal care. In some cases subjects have not been examined by dentists. These problems may be compounded if the prevalence of the disease is low and sample sizes are small. More appropriate methodologies and standardised diagnostic criteria may be required before details of the incidence and prevalence of HIVassociated periodontal diseases can be assessed.

\section{Microbiology}

The use of antimicrobial drugs for other HIV-related conditions creates difficulty investigating the microbiology of
HIV-associated periodontal diseases. Despite this, culture techniques, indirect immunofluorescence (Murray et al. 1988. Murray et al. 1989), indirect ELISA (Murray et al. 1987, Greve et al. 1988) and DNA probes (Murray et al. 1991) have been used with some success at UCSF. The microflora of both HIV$G$ and HIV-P was found to be similar and comparable to that of classical periodontitis. In particular Porphyromonas gingivalis (formerly Bacteroides gingivalis), Bacteroides intermedius, Fusobacterium nucleatum, Actinobacillus actinomvcetemcomitans, Eikenella corrodens and Wolinella species (particularly $W$. recta) were identified. Although large numbers of the organisms were not identified. the group stressed that the levels of spirochaetes present in the lesions were variable and not consistent with the microflora associated with ANUG (Murray et al. 1988).

The subgingival microflora from HIV-periodontitis sites have also been examined by Rams et al. (1991). In a study involving 14 subjects, healthy sites were compared with sites diagnosed with the disease. The predominant cultivable microflora of the HIV-P sites were gram-negative anaerobes including $W$. recta. $B$. intermedius and $A$. actinomicetemcomitans and the gram positive anaerobe Peptostreptococcus micros. The authors also likened this pattern of microflora to that of conventional periodontitis in otherwise normal subjects.

Zambon et al. (1990) have cultivated the predominant subgingival microflora from 11 patients with AIDS and moderate periodontitis. Immunofluorescence microscopy was also used to determine the presence of putative periodontal pathogens in 50 subjects (6. with a reported history of ANUG. 7 with diagnosed HIV-gingivitis and 1 with diagnosed HIV-periodontitis). 43 bacterial species were identified including many of the Gram-negative anaerobic organisms associated with conventional periodontitis. These findings are compatible with those of Murray et al. (1989) although A. actinom!cetemcomitans was detected in small numbers. and only by immunofluorescence. The authors account for this discrepancy with the findigns of Murray et al. (1989) by pointing out that only one patient in their sample exhibited HIV-periodontitis.

Lucht et al. (1990) detected no significant differences between the microflora of the saliva or gingival crevices of 30 people with HIV infection (10 with no clinical signs, 10 with ARC and 10 with AIDS) compared to a control group. The subjects in this study had all undergone dental treatment before enrolment in the study and were considered to have a 'healthy dental status'. This may have contributed to discrepancy between these findings and those of the other studies.

In a 2nd report, Lucht et al. (1991) recorded the subgingival microflora. numbers of lymphocyte subsets and periodontal indices for a very similar group of 40 people. In these patients. no association was found between periodontal health and amounts of visible plaque or counts of $A$. actinomycetemcomitans, Capnocytophaga spp. and $P$. gingivalis. Increased gingival bleeding and pocketing was found to be associated with more severe HIV disease. Pocket depth and numbers of pockets were negatively correlated with peripheral T4 lymphocyte counts. A diagnosis of HIV-periodontitis was not made for any of the subjects of this study and the increased pocketing reported contrasts with the clinical features of HIV-periodontitis described by Winkler et al. (1983a). It should be noted that the community periodontal index of treatment needs used by this group does not record gingival recession and is unsuitable for the measurement of a disease where recession may be a key feature (Ainamo et al. 1982).

Zambon et al. (1990) have also cultivated Clostridium difficile, Clostridium clostridiiforme, Enterococcus faecalis, Enterococcus avium and Klebsiella pneumoniae from the subgingival plaque of people with AIDS. These organisms are not widespread in the subgingival plaque of people without AIDS. Likewise. 12 of the 14 subjects studied by Rams et al. (1991) harboured a range of unusual organisms including Enterobacteria spp. Pseudomonas aeruginosa. C. albicans and Klebsiella spp.

Murray et al. (1988) noted that HIVgingivitis and HIV-periodontitis sites harboured significantly more Candida albicans than control sites. Zambon et al. (1990) detected yeasts in the subgingival plaque of 31 of $50(62 \%)$ patients with AIDS. Samples were speciated for 9 of these patients and Candida albicans was identified in all 9 cases. This carriage rate is much higher than the rate reported in adults with severe periodontitis (Slots et al. 1988). Zambon et 
al. (1990) associated this finding with the immunosuppression of AIDS but did not report whether their subjects were using any antimicrobial medications which might affect their oral microflora.

The significance of these findings is unclear and several explanations for the aetiology of HIV-associated periodontal diseases remain. The diseases may result from infection by the organisms associated with conventional periodontitis whose virulence is increased with the diminished immune response associated with HIV disease. Alternatively, the unusual organisms detected in people with HIV infection may contribute to the disease.

\section{Natural History}

No clear evidence is available on the links between HIV-gingivitis and HIVperiodontitis. Murray et al. (1988) have suggested that HIV-G represents an early stage of HIV-P with the subgingival microflora remaining relatively avirulent until a decrease in the ratio of T4/T8 lymphocytes allows progression of the disease. In one study of 113 patients (42 with AIDS, 23 with ARC and 40 seru-pusitive men without ARC/ AIDS) attending UCSF, HIV-G was diagnosed in patients with normal T4/T8 cell ratios. HIV-P was found only in men with low T4-/T8 cell ratios and was found more frequently in patients with Oral Hairy Leukoplakia and Oral Candidiasis (Winkler et al. 1988a). Baseline data on this cohort were reported in 1988 (Winkler et al. 1988b), but as yet no follow up data has been reported. However. Grassi has monitored 12 patients with HIV-G for $I$ year, none of whom developed HIV-P (Grassi et al. 1990).

\section{Management Regimes}

The UCSF team have widely reported an evaluation of three management regimes for HIV-P based on a study of 30 patients (Grassi et al. 1988a, 1988b.c). All patients received oral hygiene instruction. scaling and root planing. In addition 6 patients were asked to irrigate their gingivae with povidone-iodine from a syringe 3 to $5 \times$ daily for 3 months. A 3rd group of 10 patients were asked to rinse with $15 \mathrm{ml}$ of $0.12 \%$ chlorhexidine solution $2 \times$ daily for 3 months. Only the patients using the chlorhexidine rinses showed any im- provement in periodontal health. More recently, Winkler et al. (1989a) have recommended a more detailed regime, based on their experiences at UCSF which is yet to be formally evaluated. This regime includes the following.

(i) Debridement of the lesions to mechanically remove plaque, calculus and necrotic tissue. Frequent irrigation with Povidone lodine during this phase is said to have a topical anaesthetic effect and help control bleeding (Grassi et al. 1988c).

(ii) Meticulous oral hygiene is stressed. Dentists or hygienists instruct patients in the use of interproximal cleaning devices where ever tissue destruction has made oral hygiene difficult.

(iii) Patients are prescribed $250 \mathrm{mg}$ oral metronidazole $4 \times$ a day for 4 or 5 days and $0.12 \%$ chlorhexidine mouthwash twice daily. Antifungal therapy is prescribed if required.

(iv) After the acute phase of treatment, frequent follow-up is needed to monitor compliance with oral hygiene advice and for root planing to prevent relapse.

\section{Conclusions}

Although periodontal diseases have been established as part of the spectrum of conditions associated with HIV infection (Pindborg 1988 etc.), the links remain between them remain unclear. HIV-related periodontal diseases undoubtedly exist and can be severe in some patients, yet relatively little is known about their prevalence and progression.

Carefully constructed studies using appropriate sampling techniques and control populations are required to investigate the epidemiology of the disease and its natural history: Larger randomised controlled trials are also needed to test the efficacy of the treatment regimes for the diseases.

The establishment of defining criteria would allow all forms of the disease to be recognised and enable the collection of precise prevalence data and comparison of results between research centres. This should demonstrate any geographical differences in the form and distribution of the disease and will be necessary if the disease is uncommon so that its prevaience can be estimated accurateiy.

\section{Acknowledgements}

The author wishes to thank Dr. Helen Donoghue, Prof. Aubrey Sheiham, Ms. Julie Weeks and Dr. Joanna Zakrewvska for reading drafts of this manuscript, for their helpful suggestions and for their encouragement.

\section{Zusammenfassung}

Parodontalkrankheiten und HIV-Infektion. Eine Literaturstudie

Im Schriftum wird der Zusammenhang zwischen HIV-Infektion und parodontalen Erkrankungen oft erwähnt. Obwohl nur mangelhaft unterbaut, scheint er als ein Teil der zu erwartenden HIV-Folgen akzeptiert zu werden. Originalstudien haben zu widersprüchlichen Ergebnissen geführt. Als Grund dafür könnten Mängel der Versuchsanordnung infrage kommen. Hinsichtlich Klassifikation, Epidemiologie, Mikrobiologie, Pathogenese und Behandlung HIV-abhängiger Parodontalkrankeiten verbleibt darum das Bild unübersichtlich. Bei zukünftigen Studien sollte den Auswahlmethoden, dem Einsatz von Kontrollgruppen und den Definitionskriterien mehr Aufmerksamkeit gewidmet werden. Das würde Vergleiche der Versuchsdaten zwischen Forschungszentren ermöglichen und das Studium einer vielleicht nur selten vorkommenden Krankheit erleichtern.

\section{Résumé}

Les maladies parodontales et le SIDA: une revue de la littérature

La littérature contient beaucoup de réferences de maladies parodontales associées au SIDA. Quoique mal étayées, ces références les classeraient en tant que partie de la variation attendue dans les conditions associees au SIDA. Les études originales ont produit des rapports incompatibles, résultant peut-ètre de défauts de design. Par conséquent, la situation est encore confuse en ce qui concerne le classement. l'épidémiologie, la microbiologie. ['histoire naturelle et le traitement des maladies parodontales associées au SIDA. A l'avenir, il faudrait que les études s'occupent des méthodes de sondage, de l'utilisation des cas tèmoins et de la définition des critères. Cela faciliterait la comparaison des donnees entre les centres, et l'étude d'une maladie peut-ètre assez rare.

\section{References}

Abel. S. N. \& Andriolo. M. (1989) Clinical management of HIV related periodontitis: report of a case. Journal of the American Dental Association 119 (suppl.), 35S-36S.

Akula. S.. Creticos, C. \& Weldon-Linne. M. (1989) Gangrenous stomatitis in AIDS. Lancet 1 (ii), 955, (Letter).

Anon. (1988) Periodontal disease and AIDS. New York Dental Journal 58, 152. 
Ainamo, J., Barmes. D.. Beagrie. G.. Cutress. T.. Martin, J. \& Sardo-Infirri, J. (1982) Development of the World Health Organisation (WHO) community periodontal index of treatment needs (CPITN). International Dental Journal 32. 281-291.

Apaiza. T.. Franco. F. \& Pebollo. J. (1991) Periodontal status and subgingival microflora in HIV $(+)$ patients. Clinical report. Journal of Dental Research. 70. (abstr.) 415.

Barr. C.. Rua-Dobles. A.. Andersson. M. R. \& Croxson, T. (1990) Periodontal disease: Oral indices. immunologic profile and HIV serostatus. Journal of Dental Research 69, (abstr.) 186.

Barr. C.. Rua-Dobles. A. \& Andersson, M. R. (1991) Periodontal disease by HIV status in a gay male cohort. Journal of Dental Research 70. (abstr.) 415.

Cogen. R. B. (1990) Acute necrotizing ulcerative gingivitis. In: Contemporary periodontics, eds. Genco. R. J., Goldman. H. M. \& Cohen. D. W.. pp. 459-65. St. Louis: C. V. Mosby Company.

Cornick. D. E. R. \& Robinson, P. G. (199I) A three year study of the periodontal condition in HIV positive men. Journal of Dental Research 70. (abstr.) 688.

Dennison, D. K., Smith. B. \& Newland, J. R. (1985) Immune responsiveness and ANUG. Journal of Dental Research 64, (abstr.) 204.

Ducka-Karsa. K. (1979) Affections buccodentaires dans la population de Lubumbashi (Zaire). Tropical Dental Journal 8, $15-17$.

EEC Clearinghouse on oral problems related to HIV infection \& WHO Collaborating Centre on oral manifestations of the Human Immunodeficiency Virus. (1991) An update of the classification and diagnostic criteria of the oral lesions in HIV infection. Journal of Oral Pathology and Medicine 20. 97-100.

Emslie. R. D. (1963). Cancrum Oris. Dental Practitioner 13, 481-495.

Farthing. C. (1986) AIDS. The dental picture. Dental Practice 24. 1-2.

Farthing. C.. Brown. S. \& Staughton. R. (1988) An Atlas of .tIDS, 2nd edition, p. 35. Lundon: Wolfe.

Felix. D. H.. Wray. D.. Smith. G. L. F. \& Jones. G. A. (1991) Oro-antral fistula: an unusual complication of HIV-associated periodontal disease. British Dental Journal 171, 61-62.

Genco, R. J. (1990) Classification and clinical and radiographic features of periodontal disease. In: Contemporary periociontics, eds. Genco. R. J.. Goldman. H. .I. \& Cohen. D. W.. pp 68-9. St. Louis: C. V. Mosby Companv.

Genco. R. J. \& Slots, J. (1984) Host responses in periodontal disease. Journal of Dental Rescurch 63. $+41-4+8$.

Giovanni. M.. Zuccoti. G. V. \& Fiocchi, A. (1989) Gangrenous stomatitis in a child with AIDS. Lancet. 2(ii), 1400 (letter).

Gornitsky, M. \& Pekovic. D. (1987) Involve- ment of HIV in gingiva of patients with AIDS. Advances in Experimental Medicine and Biology 21. 553-562.

Grassi. M., Hammerle. C., Staschek. I., Berthold. H. \& Lang. N. P. (1990) Longitudinal observation of HIV-associated gingivitis. 6th International Conference on AIDS San Francisco 1. 103 (poster).

Grassi. M.. Williams. C. A., Winkler. J. R. \& Murrav. P. A. (1988a) Local treatments of HIV associated periodontal disease. $4 \mathrm{th}$ International Conference on AIDS Stockholm. p. 319 (abstr.)

Grassi. .M.. Williams. C. A., Winkler. J. R. \& Murrav. P. A. (1988b) Local treatments of HIV associated periodontal disease. Journal of Dental Research 67. (abstr.) 117.

Grassi. M.. Williams. C. A., Winkler. J. R. \& Murrav. P. A. (1988c) Management of HIV-associated periodontal diseases. In: Perspectives on oral manifestations of AIDS. eds. Robertson. P. B. \& Greenspan, J. S.. pp. 119-130. Littleton: PSG Publishing Company. Inc.

Greenspan. J., Greenspan. D., Pindborg. J. \& Schiodt. M. (1986) AIDS and the dental team. Ist edition. pp. 42-44. Copenhagen: Munksgaard.

Greve. W. G.. Winkler. J. R. \& Murray. P. A. (1988). HIV sero-positive individuals humoral immune response to periodontal pathogens in HIV-P. 4 th International Conference on AIDS. Stockholm. p. 318 (abstr.).

Henshaw. N. E. \& Adenjubi. J. O. (1975) Periodontal disease in northern zone of Nigeria. Nigerian Medical Journal 5, 152-159.

Lucht. E.. Teivens. A.. Berglund. O.. Heimdahl. A. \& Nord. C. E. (1990) Occurrence of micro-organisms in saliva and gingival crevice in HIV-1-infected patients. .Mircobial Ecology in Health and Disease 3. 329-334.

Lucht. E.. Heimdahl. A. \& Nord. C. E. (1991) Periodontal diseases in HIV-infected patients in relation to lymphocyte subsets and specific microorganisms. Journal of Clinical Periodontology 18. 252-256.

Melnick. S. L., Engel. D.. Truelove. E.. DeRouen. T.. Morton. T.. Schubert. M.. Dunphy. C. \& Wood. R. W. (1989) Oral mucosal lesions: Association with the presence of antibodies to the humanimmunodeficiency virus. Oral Surgers: Oral Medicine. Oral Pathology 68. $37-43$.

Murrav. P.. Fenesv. K.. Scorzello. T.. Linch. M.. Najjar. T. \& Jandinski. J. (1991) HIVassociated periodontal disease in IV-Jrug users. Journal of Dental Resarch $70 .+15$ (abstr.).

Murray: P. A.. Grassi. M. \& Winkler. J. R. (1989) The microbiology of HIV-ilssociated periodontal lesions. Journal of Clinical Poriodontology 16. 636-642

Murray. P. A.. Grassi. M. \& Winkler. J. R. (1987) The humoral immune response in HIV-associated periodontitis. 3rd Intcrnational Conference on AIDS. Washington. p. 187 (abstr.).
Murray. P. A., Winkler, J. R., Peros. W. J.. French. C. K. \& Lippke. J. A. (1991) DNA probe detection of periodontal pathogens in HIV-associated periodontal lesions. Oral Microbiology and Immunology 6. $34-40$.

Murray. P. A.. Winkler. J. R.. Sadowski, I.. Kornman. K. A.. Steffensen. B.. Robertson. P. B. \& Holt. S. C. (1988) Microbiology of HIV-associated gingivitis and periodontitis. In: Perspectives on oral manifestations of AIDS, eds. Robertson. P. B. \& Greenspan. J. S., pp. 105-108. Littleton: PSG Publishing Company, inc.

Pekovic, D. D., Lawton. R., Adamkiewicz. V. W.. Gornitsky, M. \& Ajdukovic. D. (1988) AIDS associated periodontal disease. 4th International Conference on AIDS Stockholm. p. 317 (abstr.).

Phelan. J. A.. Saltzmann, B. R., Friedland. G. H. \& Klein, R. S. (1987) Oral findings in patients with acquired immunodeficiency syndrome. Oral Surgery, Oral Medicine. Oral Pathology 64, 50-56.

Pindborg, J. J. (1988) Classification of oral lesions associated with HIV infection. Oral Surgery, Oral Medicine, Oral Patholog! 67. 292-295.

Pindborg. J. J. (1986) Acute necrotizing ujcerative gingivitis in a Danish AIDS patient. Tandlaegebladet 90, 45.

Pindborg, J. J. \& Holmstrupp, P. (1987) Necrotizing gingivitis related to HIV infection. African Dental Journal 1, 5-8.

Rams. T. E., Andriolo. M. Jr., Feik, D.. Abel. S. N.. McGivern, T. M. \& Slots, J. (1991) Microbiological study of HIV-related periodontitis. Journal of Periodontology 62. 74-81.

Rosenstein. D. I.. Eisener, T. L.. Levin. M. P. \& Chiodo. G. T. (1989) Rapidly progressive periodontal disease associated with HIV infection: report of a case. Journal of American Dental Association 118. 313-314.

Sadoun. A. P. (1987) Les maladies parodontales et al Sida. Journal de Paradontologie 6. 345-355.

SanGiancomo. T. R.. Tan. P. M.. Loggi. D. G. \& Itkin. A. B. (1990) Progressive osseous destruction as a complication of HIVPeriodontitis. Oral Surgery; Oral Medicine. Oral Pathology 70. 476-479.

Schulten. E. A. J. M.. Ten Kate. R. W. \& Van Der Wial. I. (1990) Oral findings in HIVinfected patients attending a department of internal medicine: The contribution of intratoral examination towards the clinical management of HIV disease. Quartert! Journul of Medicine 76. 741-745.

Silverman. S.. Migliorati. C. A., Lozada-Nur. F. Greenspan. D. \& Cunalt. M. A. (1986) Oral lindings in people with or at high risk for AIDS: a study of 375 homosexual males. Journal of the American Dental A.ssociation 112. 187-193.

Slots. J., Rams. T. E. \& Listgarten. M. A. (1988) Yeasts. enteric rods, and pseudomonads in the subgingival flora of severe 
adult periodontitis. Oral Microbiology and Immunology 3, 47-52.

Smith, G. L. F., Felix. D. H. \& Wray, D. (1991) HIV associated periodontal disease: prevaience, clinical features and management. Journal of Dental Research 70, 688 (abstr.).

Sznajder. N.. Carraro. J. J.. Rugna, S. J. \& Sereday, M. (1978) Periodontal findings in diabetic and non-diabetic patients. Journal of Dental Research 49. 445-448.

Tukutuku. K., Muyembe-Tamn. n, L.. Kayembe. K., Mavuemba. T., San ra. N. \& Sekele. I. (1990) Prevalence of dental caries, gingivitis, and oral hygiene in hospitalized AIDS cases in Kinshasa, Zaire. Journal of Oral Pathology and Medicine 19, 271-272.

Williams. C. A., Winkler. J. R., Grassi,97.\& Murray, P. A. (1990) HIV-associated periodontitis complicated by necrotizing stomatitis. Oral Surgern, Oral Medicine, Oral Pathology 69, 351-355.

Winkler. J. R., Grassi. M. \& Murray, P. A. (1988) Clinical description and etiology of HIV-associated periodontal diseases. In:
Perspectives on oral manifestations of AIDS. eds. Robertson. P. B. \& Greenspan, J. S., pp. 49-70. Littleton: PSG Publishing Company, inc.

Winkler. J. R., Grassi, M.. Williams, C. A. \& Murray. P. A. (1988) Clinical documentation of HIV associated periodontitis and gingivitis. 4th International Conference on AIDS. Stockholm. p. 317 (abstr.).

Winkler. J., Herrera. C.. Nathan, R.. Williams. C.. Buchbinder. S.. Hessol, N.. Lifson. A. \& Lee. K. (1990) Correlation of immune and periodontal status in homosexual men. Journal of Dental Research 69 , 221 (abstr.).

Winkler. J. R. \& Murray, P. A. (1987) A potential intra-oral expression of AIDS may be rapidly progressive periodontitis. Journal of the California Dental Association 15. 20-24.

Winkler. J. R., Murray. P. A., Grassi, M. \& Hammerle, C. (1989a) Diagnosis and management of HIV associated periodontal lesions. Journal of the American Dental Association 119 (suppl.), 25S-34S.

Winkler. J. R., Murray, P. A., Greenspan,
D. \& Greenspan. J. A. (1986) Periodontal disease of male homosexuals as related to AIDS virus infection. 2nd International Conference on AIDS, Paris, p. 43 (abstr.).

Winkler, J. R., Murray, P. A. \& Hammerle. C. (1989b) Gangrenous stomatitis in AIDS. Lancet 2(i), 108 (letter).

Zakarian, A., Andriolo. M., Abel, S. \& McGivern, T. (1988) HIV-related ulcerative gingivitis and periodontitis. 4 th International Conference on AIDS, Stockholm, p. 318 (abstr.).

Zambon. J. J.. Reynolds. H. S. \& Genco. R. J. (1990) Studies of the subgingival microflora in patients with Acquired Immunodeficiency Syndrome. Journal of Periodontology 61, 699-704.

Address:

Peter Robinson

University College Dental Hospital

London WCIE $6 A U$

$U K$ 


\title{
The periodontal health of homosexual men with HIV infection: a controlled study
}

\author{
PG Robinson'1, A Sheiham¹, SJ Challacombe², JM Zakrzewska ${ }^{1}$ \\ ${ }^{1}$ Department of Epidemiology and Public Health, University College, London, WC1E 6BT; ${ }^{2}$ Department of Oral Medicine and \\ Pathology, United Medical and Dental Schools, London, SE1 9RT, UK
}

\begin{abstract}
OBJECTIVE: Identify types, prevalence and severity of periodontal changes associated with HIV infection.

DESIGN: Cross-sectional controlled blinded study. SETTING: Open access genito-urinary medicine clinic. PARTICIPANTS: Convenience sample of 794 homosexual men aged 18-65.
\end{abstract}

OUTCOME MEASURES: Prevalence, extent and severity of probing attachment loss (PAL), pocketing, gingival ulceration, gingivitis, bleeding on probing (BOP), gingival red bands and diffuse and punctate erythema of the attached gingiva (selected a priori).

RESULTS: Prevalences in men with $(n=312)$ and without HIV $(n=260)$ were: PAL ( $\geqslant I$ site $\geqslant 4 \mathrm{~mm}), 59.6 \%$ and $28.5 \%$ respectively $\left(P<0.00 I, \chi^{2}\right)$; pocketing $(\geqslant$ I site $\geqslant 4 \mathrm{~mm}) 5 \mathrm{I} .0 \%$ and $31.9 \%(P<0.00 \mathrm{I})$; BOP, $96.5 \%$ and 92.3\% $(P=0.038)$; gingival ulceration, $3.2 \%$ and $1.0 \%(P=$ $0.03 \mathrm{I})$, red banding, $12.2 \%$ and $10.0 \%(P=0.410)$; diffuse erythema, $12.5 \%$ and $3.1 \%(P<0.00 I)$ and punctate erythema, $9.6 \%$ and $1.1 \%(P<0.001)$. Decreased CD4 lymphocyte counts predicted the presence, extent and severity of PAL $(P=0.023,0.027$ and 0.060$)$ but not pocketing. Oral candidiasis predicted the extent and severity of gingivitis and the presence of diffuse and punctate erythema $(P=0.037,0.011,0.002$ and $<0.001)$.

CONCLUSIONS: Destruction of periodontal attachment is associated with progression of HIV disease whereas pocketing is associated with HIV infection but not disease progression. Gingival ulceration is associated with HIV but gingivitis and erythema of the attached gingiva are most strongly associated with oral candidiasis. Gingival red bands were not associated with HIV infection.

Keywords: HIV seropositivity; opportunistic infections; periodontal diseases; gingivitis; epidemiology; fungal diseases

A number of periodontal changes have been associated with $\mathrm{HIV}$ infection and classified by the EC Clearinghouse on oral problems related to HIV infection (EC-Clearinghouse

\footnotetext{
Correspondence: PG Robinson, Department of Oral Medicine and Pathology, United Medical and Dental Schools, London Bridge, London, SE1 9RT, UK

Received 3 November 1995; revised 5 January 1996; accepted 14 January 1996
}

and WHO Collaborating Centre, 1993). A distinct fiery red band along the margin of the gingiva disproportionately intense for the amount of plaque has been called linear gingival erythema. Ulcero-necrotic disease may be limited to the interdental papillae (necrotising gingivitis) or may be more extensive, perhaps involving exposure, destruction or sequestration of bone (necrotising periodontitis). Very extensive lesions of the oral mucosa which expose underlying bone or penetrate contiguous tissues may extend from areas of necrotising periodontitis and are termed 'necrotising stomatitis'.

Other changes have been reported. Winkler et al (1989) described punctate or diffuse erythema of the attached gingiva and rapidly progressive periodontitis has also been described in a patient with HIV (Rosenstein et al, 1989).

Very little is known of these conditions and methodological issues may have hindered progress. Epidemiological research often requires randomly selected study groups of adequate size, control groups and some attempt to blind examiners to the nature of participants. Where measures or indices of health are used, they should be defined and be sensitive to the types of changes expected in participants. In studies where diagnoses are assigned to subjects, explicit, valid, reliable, unambiguous and mutually exclusive diagnostic criteria are required. Much research of periodontal changes associated with HIV has failed to meet these criteria and this may account for conflicting reports of the nature and frequency of these changes (Robinson, 1992; Robinson et al, 1994; Lamster et al, 1995). Thus, this study aimed to assess the types, prevalence and severity of periodontal changes in people with HIV infection and to identify factors that may have caused the changes.

\section{Subjects and methods}

A convenience sample of homosexual men attending a genito-urinary medicine clinic in London was examined during a 15 -month period. All were between 18 and 65 years of age, had 20 or more teeth, did not have a history of injected drug use and did not have medical conditions which precluded periodontal examination. Men with Downs syndrome, diabetes or leukaemia or those taking epanutin or cyclosporins were also excluded. Men meeting the inclusion criteria were identified by the clinic staff and recruited to the study. All participants gave written consent.

The annual incidence of gingival ulceration among 
people with HIV is approximately $2 \%$ with a significant relapse rate (Robinson and Cooper, 1993; Glick et al, 1994). It was estimated that this might result in moderate attachment loss in $10 \%$ of men in late HIV disease, in addition to background levels of disease evident in adults (Brown et al, 1989; Todd and Lader, 1991). A sample of 300 men was chosen to give an $85 \%$ power to detect a difference between proportions of 0.1 and 0.2 between 'early' and 'late' HIV disease for an alpha of 0.05 (Altman, 1991). Estimates based on the prevalence of HIV infection among homosexual men at the clinic suggested that a convenience sample of 800 homosexual men would include 300 men with HIV and a similar number of men known not to be infected.

A piloted, self-complete questionnaire which included demographic variables, reason for the visit, whether rightor left-handed, tobacco use and other non-injected recreational drug use was administered to all participants. The questionnaire was then used as a structured interview guide to ensure that it had been completed fully.

To allow the examiner to be blinded to the HIV status of participants, clinical examinations took place in a separate room and participants were asked not to reveal information about their status to the examiner. A single examiner was used throughout the study (PR). Examinations were carried out with participants lying on a couch with their mouths illuminated by a Daray portable halogen lamp (Daray Lighting Ltd, Leighton Buzzard, UK) held $60 \mathrm{~cm}$ away. Periodontal measurements were made with a Michigan probe (Hu-Friedy Mfg Co, Leimen, Germany; diameter $0.6 \mathrm{~mm}$ ) with Williams markings at a force of $0.25 \mathrm{~N}$.

The presence of mucosal diseases associated with HIV infection was recorded for each participant using the presumptive diagnostic criteria devised by the US collaborative group (Greenspan et al, 1992).

Periodontal examinations were conducted for buccal sites of all teeth on a half mouth basis, alternating right and left sides. The periodontal measures and indices for each tooth comprised:

i) Plaque score for each tooth using the plaque index of Silness and Loe for the mid-buccal and mesio-buccal sites (Silness and Loe, 1964)

ii) Appearance of marginal gingiva using the gingival index of Loe and Silness with an additional category to record a bright red band (Loe and Silness, 1963):

$\begin{array}{ll}\text { Score } & \begin{array}{l}\text { Signs } \\ 0\end{array} \\ 1 & \begin{array}{l}\text { Normal gingiva } \\ \text { Mild marginal inflammation, slight change in col- } \\ \text { our, slight oedema }\end{array} \\ 2 & \begin{array}{l}\text { Moderate marginal inflammation, redness and } \\ \text { glazing }\end{array} \\ 3 & \begin{array}{l}\text { A distinct bright red band along the marginal gin- } \\ \text { giva from papilla to papilla } \\ \text { Severe inflammation-marked redness and oed- } \\ \text { ema, with ulceration or spontaneous bleeding }\end{array}\end{array}$

Only categories 0,1 and 2 were included when considering the severity of gingivitis. Analyses for red banding and ulceration were conducted independently. iii) Appearance of attached gingiva by the presence of Punctate erythema or Diffuse erythema

iv) Presence of visible bone

v) Probing depths were measured at the disto-buccal, midbuccal and mesio-buccal sites

vi) Presence or absence of bleeding for each site probed vii) Distance between the cemento-enamel junction and the gingival crest at the disto-buccal, mid-buccal and mesiobuccal sites. This measure along with the probing depth allowed the calculation of the probing attachment loss using the Ramfjord method (Ramfjord, 1967). Where the cemento-enamel junction (CEJ) was obscured by restorations or calculus the apical extent of the restoration or calculus was used as the reference point.

The presence or absence of the characteristic foetor of acute necrotising ulcerative gingivitis was also recorded for each participant. Medical data were collected from participant's medical records one month after the visit to allow the findings of laboratory investigations carried out on that day to be entered. The following medical data were recorded for each participant:

i) HIV sero-status

ii) Clinical stage of HIV disease using the revised 1993 classification system for HIV disease (Centers for Disease Control and Prevention, 1992)

iii) CD4+ve, CD8+ve and total lymphocyte counts (for HIV antibody-positive men)

iv) Neutrophil count

v) Current and recent medications (medications first prescribed on the day of the visit were ignored).

All haematological values used were recorded within 6 weeks of the examination except for a small number of men with AIDS who had not had CD4+ve lymphocyte counts for up to 8 months. In this case the most recent count was used (all were under 50 cells $\mathrm{mm}^{-3}$ ). Medical records of men not tested for HIV antibodies at the time of their examination were viewed again at the end of the study to ascertain whether they had been tested subsequently.

\section{Data analysis}

Summary measures were created to capture the presence, extent and severity of periodontal conditions without analysis of multiple non-independent observations using the individual as the unit for analysis (Imrey, 1986). A condition was defined as present if there was one or more sites with the condition. The extent of a condition was defined as the proportion of sites affected within each individual and the severity of the condition was defined as the maximum score or index for the condition within each individual.

The data were skewed for six variables and were transformed to allow parametric analyses. Maximum probing attachment loss (PAL) was transformed to $\log$-maximum PAL. Very few men had probing depths or PAL of $4 \mathrm{~mm}$ or greater so that a dichotomous variable was created which recorded whether men fell into the top quartile by the proportion of sites with probing depths or attachment loss greater than $4 \mathrm{~mm}$. The extent of gingivitis was converted into a categoric variable by the presence or absence of gingivitis at all teeth. The extent of bleeding on probing (BOP) was transformed into $\sqrt{ }$ (proportion of sites with BOP). CD4+ve $T$ lymphocyte counts were transformed to $\sqrt{ }(\mathrm{CD} 4+\mathrm{ve}$ 
counts). Data were normally distributed for all other variables and fulfilled the criteria for parametric and regression analyses.

Analysis was carried out in three phases. Initially, descriptive statistics and univariate analyses $\left(\chi^{2}\right.$ tests, Student's $t$-tests and Mann-Whitney Wilcoxon Tests) were used to describe and compare key descriptor variables and distributions of summary measures of periodontal health in men with and without HIV.

The second phase aimed to identify periodontal changes associated with HIV infection. Scatter plots were drawn for the presence of outliers in the data and to assess possible linear relationships. Correlation, Student's $t$-tests and linear regression were used in univariate analyses to assess the relationship between outcomes and the following independent variables: age, HIV status, the proportion of surfaces with plaque, whether the participant held his toothbrush in the ipsi- or contra-lateral hand, race, socioeconomic status, medications, alcohol (dichotomised around the median level of consumption) and the following recreational drugs (all recorded as ever or never): tobacco, cannabis, nitrite inhalants, amphetamines, cocaine, heroin and 'others'. Those independent variables for which the null hypothesis had a probability of less than 0.2 were tried in forward stepwise regression models (JMP 2.0, SAS Institute Inc, Cary, NC, USA). Multiple regression analysis was used for continuous outcome variables and logistic regression for dichotomous outcomes $\left(R^{2}\right.$, the proportion of the variation in the data explained by the model tends to be smaller in logistic regression).

Only men with HIV infection were included in the final phase which aimed to identify possible mediators of the association between HIV and changes in periodontal health. Independent variables specific to men with HIV or only available for men with HIV were introduced and tested in correlation and univariate analyses. These were: CD4+ve and CD8+ve and total lymphocyte counts; neutrophil counts; clinical staging of HIV disease; use of HIV-related medications (specifically: Cotrimoxazole, Pentamidine, Dapsone, Nystatin. Amphotericin, Miconazole, Ketoconazole, Fluconazole, Azidothymidine, ddI, ddC, Acyclovir, Gancyclovir, Prednisolone or 'others' all dichotomised by whether each participant had taken the drug in the last 3 months) and the presence of the following oral manifestations of HIV: oral candidiasis; oral hairy leukoplakia; Kaposi's sarcoma; other oral manifestations and any oral manifestation. Associations with $P<0.2$, as well as significant variables in the second phase were tested in forward stepwise multiple and logistic regression models.

\section{Examiner reliability}

To assess the reproducibility of clinical measures examinations were repeated on 73 men $(9.2 \%)$ at the same visit. The standard deviation of repeated measures of probing attachment loss (PAL) was 0.73 and when PAL was dichotomised ( $<4 \mathrm{~mm}$ or $\leq 4 \mathrm{~mm}$ ) kappa was 0.84 . Weighted kappa for plaque scores was 0.76 and as a dichotomous variable (plaque present/absent) kappa was 0.79 . Weighted kappa for gingival index was 0.87 and kappa for BOP was 0.70 , all of which were considered to be within acceptable levels.

\section{Results}

Complete data were collected for 794 men. Of these, 260 had tested negative for HIV antibodies in the year preceding or at any time since the examination and 312 men were HIV antibody-positive. The remaining 222 did not meet either criterion and were regarded as untested.

The men with HIV (mean age, 35.9 years, $95 \%$ central range 23.8-54.2 years) were slightly older than those without (31.2 years, 95\% CR 19.5-57.5 years, $P<0.001$, Mann-Whitney Wilcoxon Test) and fewer were students $\left(2.2 \%\right.$ vs $\left.10.4 \%, P<0.001, \chi^{2}\right)$. Both groups had similar numbers of teeth (means: HIV+ve $=13.6$; HIV-ve $=13.8$, $P=0.229$, MWWT), similar mean plaque scores (HIV+ve $=1.05, \mathrm{HIV}-\mathrm{ve}=1.00)$ and similar proportions of surfaces with detectable plaque (means: $\mathrm{HIV}+\mathrm{ve}=0.52(0.47-0.58$, $95 \%$ confidence interval $) ; \mathrm{HIV}-\mathrm{ve}=0.50(0.47-0.53,95 \%$ CI), $P=0.306, t$-test).

More men with HIV visited the genito-urinary medicine clinic because of problems with their mouth or gums (25 $(8 \%)$ and $9(3.5 \%)$ respectively; $\left.P=0.042, \chi^{2}\right)$. Fourteen men $(4.5 \%)$ with HIV and eight men $(3.1 \%)$ without said they were visiting the clinic because of problems with their mouths and four HIV+ve men $(0.9 \%)$ were visiting because of gum problems compared to one HIV-ve man $(0.4 \%)$. Seven men $(2.2 \%)$ with HIV attended for both gum and mouth problems whilst none of the HIV-negative men did.

Seventy-one men with HIV $(22.8 \%)$ had a clinical indicator condition for AIDS. CD4+ve T lymphocyte counts were available for 276 men, of whom 118 had counts less than 200 cells $\mathrm{mm}^{-3}$. The mean and median counts were 325 and 270 cells $\mathrm{mm}^{-3}$ respectively. One hundred and sixty-eight men (53.8\%) met the 1993 case definition for AIDS.

One hundred and eight-six men (59.6\%) with HIV had oral manifestations of the infection. The commonest of these were: oral hairy leukoplakia, 138 (44.2\%); erythematous candidiasis, 84 (26.9\%) and pseudomembranous candidiasis 36 (11.5\%). Seven men without HIV $(2.6 \%)$ had oral lesions which met the clinical criteria for oral manifestations of HIV.

The frequencies of periodontal changes in men with and without HIV are summarised in Table 1. More men with HIV had PAL, pocketing, diffuse and punctate erythema of the attached gingiva (all $P<0.001)$, gingivitis $(P<0.038)$ and BOP $(P<0.028)$. Bright red bands were equally common in men with and without HIV.

PAL and pocketing were more severe and extensive in men with HIV. Mean maximum PAL in the men with and without HIV was $4.33 \mathrm{~mm}$ and $2.98 \mathrm{~mm}$ respectively $(95 \%$ CRs $1.8-10.0$ and $1.0-7.0$ respectively, $P<0.001, t$-test using the log transformation) and mean maximum pocket depth was $3.73 \mathrm{~mm}$ and $3.37 \mathrm{~mm}$ respectively (95\% CIs 3.51-3.86 and 3.23-3.41 respectively, $P<0.001, t$-test). The median proportions of sites with PAL in the two groups were 0.024 and 0.000 respectively ( $95 \%$ CRs 0.000 0.441 and $0.000-0.213, P<0.001$, MWWT) and the median proportions of sites with pocketing were 0.021 and 0.000 respectively $(95 \%$ CRs $0.000-0.232$ and 0.000 $0.167, P<0.001$, MWWT). The mean proportions of sites with pocketing were 0.036 and 0.019 respectively. 
Table 1 The presence of periodontal changes in homosexual men by HIV status

\begin{tabular}{|c|c|c|c|c|}
\hline Presence of: & Summary measure & $\begin{array}{c}H I V+v e \\
n(\%)\end{array}$ & $\begin{array}{c}H I V-v e \\
n(\%)\end{array}$ & $P\left(\chi^{2}\right)$ \\
\hline PAL & $1+$ site with $\mathrm{PAL} \geq 4 \mathrm{~mm}$ & $186(59.6)$ & $74(28.5)$ & $<0.001$ \\
\hline Pocketing & $1+$ site with pocket $\geq 4 \mathrm{~mm}$ & $159(51.0)$ & $83(31.9)$ & $<0.001$ \\
\hline Gingivitis & $1+$ site with gingivitis & $309(97.9)$ & $251(96.5)$ & 0.038 \\
\hline $\mathrm{BOP}$ & 1+ site with BOP & $301(96.5)$ & $240(92.3)$ & 0.028 \\
\hline Ulceration & $1+$ site with gingival ulceration & $10(3.21)$ & $5(1.04)^{\mathrm{a}}$ & 0.031 \\
\hline Red band & $1+$ site with bright red band & $38(12.2)$ & $26(10.0)$ & 0.410 \\
\hline Diffuse erythema & $1+$ site with diffuse erythema & $39(12.5)$ & $8(3.1)$ & $<0.001$ \\
\hline Punctate erythema & 1+ site with punctate erythema & $30(9.6)$ & $3(1.1)$ & $<0.001$ \\
\hline
\end{tabular}

PAL $=$ Probing attachment loss

$\mathrm{BOP}=$ Bleeding on probing

${ }^{a}$ Men not tested for HIV were regarded as HIV-ve to increase the power of the analysis for gingival ulceration only. Includes one man with ulceration not tested for HIV

Gingival ulceration, diffuse and punctate erythema of the attached gingiva and red banding were uncommon and the samples were too small to justify analysis of the extent of these variables. Gingival ulceration was present in only 15 men (10 HIV+ve, four HIV-ve and one not tested) thus samples were too small to detect differences in prevalence of the condition. Statistical power was increased by regarding 222 men not tested for HIV as HIV-ve, conferring a relative risk for gingival ulceration in HIV infection of 3.090 with a $95 \%$ confidence interval of 0.93 to 8.99 . Of the 10 men with HIV and gingival ulceration six had lesions which extended less than $4 \mathrm{~mm}$ from the CEJ, the remaining four extended $4 \mathrm{~mm}$ or more. Bone was neither visible nor palpable in any men. All 15 men with gingival ulceration had the characteristic foetor oris of acute necrotising ulcerative gingivitis. Men without gingival ulceration did not have foetor.

Of the men with HIV, $199(63.8 \%)$ had a maximum conventional gingivitis score of 2 compared to 120 (46.1\%) without HIV $\left(P<0.001, \chi^{2}\right)$. The mean proportions of sites with gingivitis among men with and without HIV were 0.777 and 0.616 respectively (95\% CRs $0.000-1.000$ and 0.138-1.000, $P<0.001$, MWWT). One hundred and twenty-five men $(40.1 \%)$ with HIV had gingivitis at all teeth compared to $46(17.7 \%)$ men without $\left(P<0.001, \chi^{2}\right)$.

Table 2 presents the best regression models for outcomes related to loss of periodontal attachment in the men of known HIV status. More surfaces with plaque, HIV infection and increased age were consistently associated with all summary measures of PAL and pocketing. Tobacco use was strongly and consistently related to PAL but not to pocketing. An apparent association between history of cocaine use and the severity of PAL was excluded in multiple regression.

Regressors for gingival outcomes are summarised in Table 3. HIV infection was strongly associated with the extent and severity of gingivitis and there was a strong tendency for HIV to be associated with the presence of gingivitis. HIV infection was also associated with the presence of bleeding on probing, gingival ulceration, diffuse and punctate erythema. Two independent variables tested in the multiple and logistic regression models were not significantly associated with gingival outcomes. These were: the use of non-HIV related medication for the extent of bleeding on probing and history of cocaine use for the presence of red bands.

Only men with HIV were included in the third phase of analysis. Table 4 summarises the best regression models for summary measures of PAL and pocketing and indicates a strong inverse relationship between $\mathrm{CD} 4+$ ve lymphocyte counts and the presence and extent of periodontal attachment loss. There was a similar trend $(P=0.06)$ for decreased CD4+ve lymphocyte counts to be associated with the severity of attachment loss.

Marginal gingivitis and bleeding on probing were so ubiquitous among men with HIV that regression analysis was inappropriate for associations with their presence. The best regression models for other gingival outcomes in men with HIV are presented in Table 5. The number of surfaces with plaque was strongly and consistently associated with summary measures of marginal gingivitis, bleeding on probing, the presence of a bright red band and the presence of diffuse erythema. Oral candidiasis was associated with measures of marginal gingivitis and with the diffuse and punctate erythema of the attached gingiva. The bright red band was only associated with the proportion of surfaces with plaque. An association between history of acyclovir use and the extent of gingivitis apparent in univariate analysis was not significant in the regression model.

\section{Discussion}

These data provide strong evidence that a number of periodontal changes are associated with HIV infection. These were: the presence, extent and severity of probing attachment loss, pocketing and conventional forms of gingivitis. Gingival ulceration, bleeding on probing and diffuse and punctate erythema of the attached gingiva were also more common in HIV disease. The regression analyses indicate that the association between HIV and these outcomes is 
Table 2 Best forward stepwise multiple and logistic regression models for outcomes related to loss of periodontal attachment in 312 men with and 260 without HIV infection

\begin{tabular}{|c|c|c|c|c|c|c|}
\hline Outcome & $\begin{array}{l}\text { Age } \\
b(\text { s.e. }) \\
P\end{array}$ & $\begin{array}{l}\text { HIV status } \\
b \text { (s.e.) } \\
P\end{array}$ & $\begin{array}{l}\text { Tobacco use } \\
b \text { (s.e.) } \\
P\end{array}$ & $\begin{array}{l}\text { Plaque } \\
b \text { (s.e.) } \\
P\end{array}$ & s.d.res & $R^{2}$ \\
\hline Presence PAL ${ }^{\mathrm{L}}$ & $\begin{array}{l}0.078(0.494) \\
<0.001\end{array}$ & $\begin{array}{l}\theta .526(0.096) \\
<0.001\end{array}$ & $\begin{array}{r}0.383(0.099) \\
<0.001\end{array}$ & $\begin{array}{l}1.007(0.393) \\
0.010\end{array}$ & & 0.149 \\
\hline Extent $\mathrm{PAL}^{\mathrm{L}}$ & $\begin{array}{r}0.078(0.013) \\
<0.001\end{array}$ & $\begin{array}{l}0.581(0.120) \\
<0.001\end{array}$ & $\begin{array}{l}0.414(0.118) \\
<0.001\end{array}$ & $\begin{array}{l}1.380(0.449) \\
0.002\end{array}$ & & 0.158 \\
\hline Severity PAL & $\begin{array}{l}0.069(0.009) \\
<0.001\end{array}$ & $\begin{array}{r}0.490(0.078) \\
<0.001\end{array}$ & $\begin{aligned} & 0.368(0.078) \\
< & 0.001\end{aligned}$ & $\begin{array}{r}1.118(0.311) \\
<0.001\end{array}$ & 1.791 & 0.228 \\
\hline Presence pocketing ${ }^{\mathrm{L}}$ & $\begin{array}{l}0.025(0.011) \\
0.021\end{array}$ & $\begin{array}{r}0.339(0.091) \\
<0.001\end{array}$ & & $\begin{array}{l}0.905(0.364) \\
0.013\end{array}$ & & 0.043 \\
\hline Extent pocketing ${ }^{\mathrm{L}}$ & $\begin{array}{l}0.028(0.012) \\
0.018\end{array}$ & $\begin{array}{r}0.368(0.109) \\
<0.001\end{array}$ & & $\begin{array}{l}1.718(0.428) \\
0.001\end{array}$ & & 0.064 \\
\hline Severity pocketing & $\begin{array}{l}0.014(0.006) \\
0.016\end{array}$ & $\begin{array}{l}0.142(0.049) \\
0.004\end{array}$ & & $\begin{array}{l}0.620(0.194) \\
0.001\end{array}$ & 1.121 & 0.051 \\
\hline
\end{tabular}

PAL $=$ Probing attachment loss

$b=$ Regression coefficient

s.e. $=$ Standard error

s.d. res $_{\text {residual standard deviation }}$

$R^{2}=$ Proportion of variation explained by the model

$\mathrm{L}=$ Logistic regression analysis (other analyses are multiple regression)

Table 3. Best forward stepwise multiple and logistic regression models for gingival health outcomes in 312 men with and 260 without HIV infection

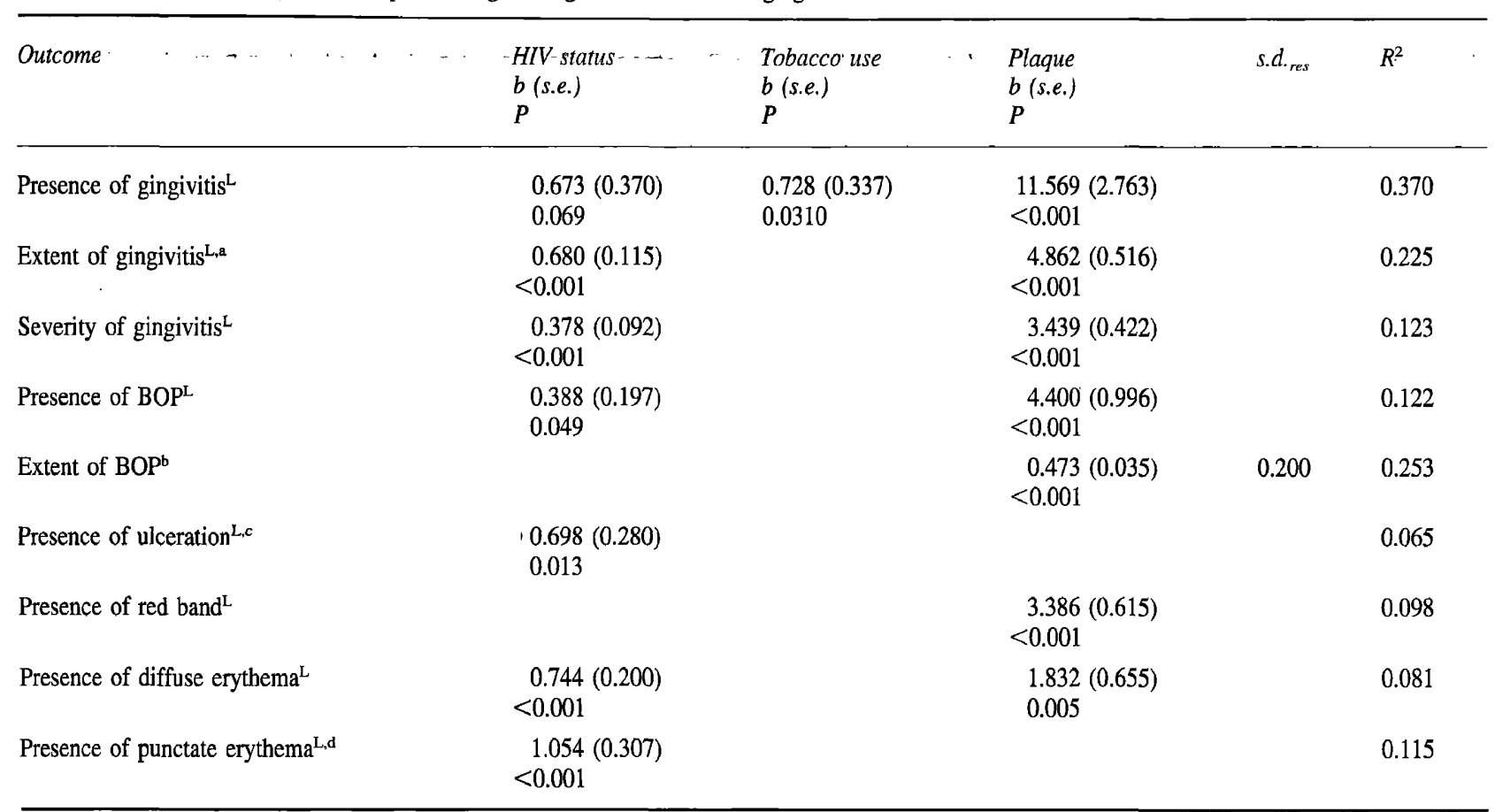

BOP = Bleeding on probing

$b=$ Regression coefficient

s.e. $=$ Standard error

s.d.res $=$ Residual standard deviation

$R^{2}=$ Proportion of variation explained by the model

$\mathrm{L}=$ Logistic regression analysis (other analyses are multiple regression)

'History of 'other drug' use was significant in the model for the extent of gingivitis. $b=0.618$, s.e. $=0.287$ and $P=0.031$

${ }^{b}$ History of nitrite inhalant use was significant in the model for the extent of BOP. $b=0.740$, s.e. $=0.009$ and $P=0.006$

${ }^{c}$ Gingival ulceration was present in only 15 men and the power of the analysis was increased by regarding men not tested for HIV as HIV-ve

${ }^{\mathrm{d}}$ History of nitrite inhalant use was significant in the model for the presence of punctate erythema. $b=0.632$, s.e. $=0.273$ and $P=0.021$ 
Table 4 Best forward stepwise multiple and logistic regression models for outcomes related to loss of periodontal attachment in 312 men with HIV infection

\begin{tabular}{|c|c|c|c|c|c|c|}
\hline Outcome & $\begin{array}{l}\text { Age } \\
b \text { (s.e.) } \\
P\end{array}$ & $\begin{array}{l}\sqrt{ } C D 4 \\
b \text { (s.e.) } \\
P\end{array}$ & $\begin{array}{l}\text { Tobacco use } \\
b \text { (s.e.) } \\
P\end{array}$ & $\begin{array}{l}\text { Plaque } \\
b \text { (s.e.) } \\
P\end{array}$ & $s . d_{\text {res }}$ & $R^{2}$ \\
\hline Presence PAL ${ }^{\mathrm{L}}$ & $\begin{array}{l}0.059(0.018) \\
0.001\end{array}$ & $\begin{array}{l}-1.327(0.584) \\
0.023\end{array}$ & $\begin{array}{l}0.530(0.141) \\
<0.001\end{array}$ & $\begin{array}{l}1.361(0.574) \\
0.018\end{array}$ & & 0.091 \\
\hline Extent PAL $^{\mathbf{L}}$ & $\begin{array}{l}0.055(0.012) \\
0.002\end{array}$ & $\begin{array}{l}-0.250(0.132) \\
0.027\end{array}$ & $\begin{array}{l}0.503(0.151) \\
<0.001\end{array}$ & $\begin{array}{l}2.201(0.590) \\
<0.001\end{array}$ & & 0.0112 \\
\hline Severity PAL ${ }^{\mathrm{a}}$ & $\begin{array}{l}0.014(0.004) \\
<0.001\end{array}$ & $\begin{array}{l}-0.250(0.132) \\
0.060\end{array}$ & $\begin{array}{l}0.122(0.030) \\
0.001\end{array}$ & $\begin{array}{l}0.310(0.117) \\
0.009\end{array}$ & 0.462 & 0.186 \\
\hline Presence pocketing ${ }^{\mathrm{L}, \mathrm{b}}$ & & & & $\begin{array}{l}1.714(0.523) \\
0.001\end{array}$ & & 0.048 \\
\hline Extent pocketing ${ }^{\mathrm{L}}$ & & & & $\begin{array}{l}<.888(0.538) \\
<0.001\end{array}$ & & 0.045 \\
\hline Severity pocketing ${ }^{c}$ & & & & $\begin{aligned} & 1.034(0.267) \\
&<0.001\end{aligned}$ & 1.089 & 0.098 \\
\hline
\end{tabular}

PAL $=$ Probing attachment loss

$b=$ Regression coefficient

s.e. $=$ Standard error

s.d.res $=$ Residual standard deviation

$R^{2}=$ Proportion of variation explained by the model

$\mathrm{L}=$ Logistic regression analysis (other analyses are multiple regression)

${ }^{a}$ Three other variables were associated with the severity of PAL in the model. These were: history of Dapsone use, $b=-0.192$, s.e. $=0.085$ and $P=$ 0.024 ; history of ddI use, $b=0.158$, s.e. $=0.081$ and $P=0.053$ and the presence of oral candidiasis, $b=-0.061$, s.e. $=0.031$ and $P=0.047$

${ }^{b}$ Two other variables were associated with the presence of pocketing: total lymphocyte count, $b=0.310$, s.e. $=0.155$ and $P=0.045$ and the presence of mucosal ulceration, $b=0.534$, s.e. $=0.271$ and $P=0.049$

cTwo other variables were associated with the severity of pocketing: total lymphocyte count, $b=0.315$, s.e. $=0.081$ and $P=<0.005$ and the presence of hairy leukoplakia, $b=-0.139$, s.e. $=0.064$ and $P=0.032$

Table 5 Best forward stepwise multiple and logistic regression models for gingival health outcomes in 312 men with HIV infection

\begin{tabular}{|c|c|c|c|c|c|}
\hline Outcome & $\begin{array}{l}\sqrt{ } C D 4 \\
b \text { (s.e.) } \\
P\end{array}$ & $\begin{array}{l}\text { Oral candida } \\
b(\text { s.e. }) \\
P\end{array}$ & $\begin{array}{l}\text { Plaque } \\
b \text { (s.e.) } \\
P\end{array}$ & s.d. $d_{\text {res }}$ & $R^{2}$ \\
\hline Extent of gingivitis ${ }^{\mathrm{L}, \mathrm{a}}$ & & $\begin{array}{l}0.304(0.146) \\
0.037\end{array}$ & $\begin{array}{r}4.849(0.698) \\
<0.001\end{array}$ & & 0.213 \\
\hline Severity of gingivitis & & $\begin{array}{l}0.357(0.140) \\
0.011\end{array}$ & $\begin{array}{l}3.718(0.613) \\
<0.001\end{array}$ & & 0.133 \\
\hline Extent of BOP & $\begin{array}{l}0.190(0.051) \\
<0.001\end{array}$ & & $\begin{array}{l}0.409(0.049) \\
<0.001\end{array}$ & 0.195 & 0.222 \\
\hline Presence of red band $d^{\mathrm{L}}$ & & & $\begin{array}{l}3.431(0.801) \\
<0.001\end{array}$ & & 0.089 \\
\hline Presence of diffuse erythema ${ }^{\mathrm{L}}$ & & $\begin{array}{l}0.560(0.178) \\
0.002\end{array}$ & $\begin{array}{l}1.987(0.767) \\
0.010\end{array}$ & & 0.077 \\
\hline Presence of punctate erythema ${ }^{L}$ & & $\begin{array}{l}0.893(0.219) \\
<0.001\end{array}$ & & & 0.144 \\
\hline
\end{tabular}

BOP = Bleeding on probing

$b=$ Regression coefficient

s.e. $=$ Standard error

s.d. .res $_{\text {r }}=$ Residual standard deviation

$' R^{2}=$ Proportion of variation explained by the model

$\mathrm{L}=$ Logistic regression analysis (other analyses are multiple regression)

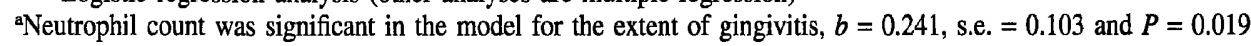

consistent and independent of other factors implicated in their pathogenesis (most notably age, plaque and tobacco use) (Tables 2 and 3 ).

The presence, extent and severity of both probing attachment loss and pocketing were increased in HIV infection.
High levels of chronic periodontitis have been found in uncontrolled cross-sectional studies of people with HIV (Riley et al, 1991; Masouredis et al, 1992) and in longitudinal studies (Barr et al, 1992; Yeung et al, 1993) but the present study finds differences in standard measures of per- 
iodontal attachment loss between HIV-positive and -negative people.

In the regression analysis all three measures of attachment loss increased with lower CD4 lymphocyte counts whereas pocketing was not related to CD4 counts (Table 4). In a similar study, Grbic et al detected neither a difference in the extent of pocketing between homosexual men with and without HIV nor more extensive pockéting in homosexual men with CD4 counts below 200 cells $\mathrm{mm}^{-3}$ (Grbic et al, 1995). The present study is larger than the study by Grbic in which 121 homosexual men and 83 drug users participated and where pocketing was considerably more extensive in all participants (mean proportions of sites with pocketing were 0.242 and 0.214 in HIV-positive and -negative men compared to 0.036 and 0.019 in this study). Grbic et al have not presented data on attachment loss or the prevalence or severity of pocketing.

In the present study, increased attachment loss may be the legacy of past episodes of ulceration which have destroyed periodontal tissue causing attachment loss without pocketing. If people with HIV have recurrent episodes of ulceration it is to be expected that the prevalence, extent and severity of such defects will increase over time as HIV disease progresses. The dramatic increases in pocketing may to some extent be ecological fallacy. Even among men without HIV, mean maximum pocket depth was $3.37 \mathrm{~mm}$, thus small increases in pocket depth, possibly due to false pocketing, would have a dramatic effect on the summary measures for extent and severity of pockets.

- False pocketing may have been due to gingivitis which was more prevalent, extensive and severe in HIV infection (Tables 1 and 3). However, gingivitis was not related to progression of HIV disease as measured by CD4 lymphocyte counts but was strongly associated with the presence of plaque and oral candidiasis. Oral candidiasis was also strongly associated with the presence of punctate and diffuse erythema of the attached gingiva. Candida species are uncommon in the sub-gingival microflora of general populations but have been detected in plaque (Murray et al, 1989; Zambon et al, 1990) and invading the gingival epithelium of people with HIV (Odden $e t a l, 1994)$. Grbic $e t$ al (1995) also reported an association betwen oral candidiasis and linear gingival erythema but did not report on erythema of the attached gingiva.

Bleeding on probing was more common in HIV infection in both univariate and regression analysis although no relationship was detected between the extent of bleeding and the infection. Among infected men bleeding was so ubiquitous (96.5\%) that regression analysis was inappropriate (this figure is almost identical to that found by Swango et al (1991). However, analysis of the men with HIV found bleeding on probing was less extensive with advanced HIV disease. One explanation might be the hypovolaemia and changes in tissue vascularity of cachexia seen in late HIV disease which might cause less bleeding.

Gingival ulceration was associated with HIV infection in both $\chi^{2}$ and regression analysis and the relative risk was 3.09 compared to other homosexual men. The $95 \%$ confidence interval for the relative risk (0.93 to 8.99) just includes one which reflects the poor statistical power for an uncommon outcome. Selection bias may have caused an overestimate of the relative risk and although compelling, these data are not conclusive evidence of an association between gingival ulceration and HIV. Three per cent of men with HIV had gingival ulceration. Allowing for selection bias this figure is comparable to findings in other studies. The annual incidence of gingival ulceration or NUP among patients at dedicated clinics has been reported at between 1 and 2\% (Robinson and Cooper, 1993; Glick et $a l, 1994)$ and $1 \%$ in a non-self-selected study population reported by Riley et al (1991). Two other studies of nonself-selected groups have reported gingival ulceration and/or cratering in 4-6\% and $25 \%$ of people with HIV (Swango et al, 1991; Masouredis et al, 1992). Different diagnostic criteria may account for the differences. For example, some participants diagnosed with HIV-P by Masouredis may not have had gingival ulceration (Masouredis et al, 1992).

Six of ten men with gingival ulcers had ulcers which extended up to $3 \mathrm{~mm}$ from the cemento-enamel junction. Three millimetres was chosen as a criterion to objectively reflect the EC clearinghouse criterion for NUG in which ulceration is limited to the inter dental papilla (EC-Clearinghouse and WHO Collaborating Centre, 1993). Using this translation, six (1.9\%) men with HIV had necrotising ulcerative gingivitis and four (1.3\%) had necrotising ulcerative periodontitis. None had necrotising stomatitis.

A bright red band of the marginal gingiva has long been associated with HIV infection (Winkler et al, 1986) but the prevalence varies between 0 and $50 \%$ in different studies (Friedman et at, 1991; Masouredis et al, 1992). In the present study the prevalence in the study and control groups was 12.2 and $10.0 \%$ respectively, prevalences similar to those found among 200 people with HIV found by Riley et al (1991). Higher prevalences were detected in two uncontrolled studies. Masouredis et al (1992) added the prevalence of red banding, diffuse and punctate erythema to create an aggregate prevalence of 31 to $50 \%$ depending on the criteria used. Forty-nine per cent of 189 HIV-positive people described by Swango had 'a continuous erythematous band at least $1 \mathrm{~mm}$ in width' and it is possible that at least some of the erythema may have been chronic marginal gingivitis. Other smaller studies have not found atypical gingivitis in HIV infection (Friedman et al, 1991; Winkler et al, 1992). In the present study, bright red bands of the marginal gingiva were strongly associated with the presence of plaque, independent of HIV status. Red bands first described by Winkler $e t a l$ and now called linear gingival erythema may be so uncommon that a sample of 312 is too small to detect any cases.

The findings of this study must be viewed with care for several reasons. Firstly, cross-sectional studies of this type are useful to identify associations between variables but do not imply cause and effect. Longitudinal and experimental studies are required to further investigate these findings.

More men with HIV were visiting the clinic with gingival problems than men without HIV $(3.1 \%$ and $0.4 \%$ respectively), thus some selection bias is present. The examinations were conducted at the genito-urinary medicine clinic to reduce bias but some people with HIV seek care from their physicians for oral problems. Selection bias may therefore have contributed to an overestimate of the 
prevalence and relative risk of acute gingival disease (especially gingival ulceration) associated with HIV infection. It is unlikely that this bias accounts for the difference in the more common chronic problems.

Sixty per cent of the men with HIV had oral manifestations of the disease. This inevitably led to a 'partial-sightedness' of the examiner and may have caused some measurement bias. It is difficult to see how this problem might be avoided. Excluding people with oral manifestations would select less immuno-suppressed subjects and would be less likely to detect differences between groups.

Many men in this study had advanced HIV infection. Fifty-four per cent met the 1993 case definition for AIDS and they would be the most likely to manifest periodontal changes associated with the disease. The prevalence, extent and severity of periodontal changes would be lower in populations with early HIV disease. However, the range of disease in this study lent greater power to the regression analyses to associate a range of periodontal changes with HIV infection and identify potential factors in their aetiology.

\section{Acknowledgements}

The authors wish to thank the staff and patients of James Pringle House, Laurie Wilkinson and Dr Wagner Marcenes for their assistance with this research. Financial support for Peter Robinson was provided by The Smithkline Beecham Research Fellowship of the Oral and Dental Research Trust.

\section{References}

Altman DG (1991). Practical statistics for medical research. Chapman and Hall: London, 611 pp.

Barr C, Lopez MR, Rua-Dobles A (1992). Periodontal changes by HIV serostatus in a cohort of homosexual and bisexual men. J Clin Periodontol 19: 794-801.

Brown LJ, Oliver RC, Loe H (1989). Periodontal diseases in the US in 1981: prevalence, severity, extent, and role in tooth mortality. J Periodontol 60: 363-370.

Centers for Disease Control and Prevention (1992). 1993 revised classification system for HIV infection and expanded surveillance case definition for AIDS among adolescents and adults. MMWR 41: 1-18.

EC-Clearinghouse on oral problems related to HIV infection and WHO Collaborating Centre on oral manifestations of the immunodeficiency virus (1993). Revised classification of oral lesions associated with HIV infection. J Oral Pathol Med 22: 289-291.

Friedman RB, Gunsolley J, Gentry A et al (1991). Periodontal status of HIV-seropositive and AIDS patients. $J$ Periodontol 62: 623-627.

Glick M, Muzyka BC, Salkin LM et al (1994). Necrotizing ulcerative periodontitis: a marker for immune deterioration and a predictor for the diagnosis of AIDS. J Periodontol 65: 393-397.

Grbic JT, Mitchell-Lewis DA, Fine JB et al (1995). The relationship of candidiasis to Linear Gingival Erythema in HIVinfected homosexual men and parenteral drug users. $J$ Periodontol 66: 30-37.
Greenspan JS, Barr CE, Sciubba JJ et al (1992). Oral manifestations of HIV infection. Oral Surg Oral Med Oral Pathol 73: 142-144.

Imrey PB (1986). Considerations in the statistical analysis of clinical trials in periodontitis. $J$ Clin Periodontol 13: 517-528.

Lamster I, Grbic J, Fine J et al (1995). A critical review of periodontal disease as a manifestation of HIV infection. In: Greenspan JS, Greenspan D, eds. Oral manifestations of HIV infection. Quintessence Publishing Co: San Francisco, pp 247-256.

Loe H, Silness J (1963). Periodontal disease in pregnancy. I. Prevalence and severity. Acta Odontol Scand 21: 551-553.

Masouredis CM, Katz MH, Greenspan D et al (1992). Prevalence of HIV-associated periodontitis and gingivitis in HIV-infected patients attending an AIDS clinic. J AIDS 5: 479-483.

Murray PA, Grassi M, Winkler J (1989). The microbiology of HIV-associated periodontal lesions. J Clin Periodontol 16: 636-642.

Odden K, Schenk K, Koppang HS et al (1994). Candida infection in the gingiva in HIV-infected persons. J Oral Pathol Med 23: 178-183.

Ramfjord SP (1967). The periodontal disease index. J Periodontol 38: $30-38$.

Riley. C, London JP, Burmeister JA (1991). Periodontal health in 200 HIV-positive patients. J Oral Pathol Med 21: 124-127.

Robinson PG (1992). Periodontal diseases and HIV infection. $J$ Clin Periodontol 19: 609-614.

Robinson PG, Cooper H (1993). Evaluation of 83 cases of gingival ulceration associated with HIV infection. Second International Workshop on the Oral Manifestations of HIV infection. San Francisco, CA.

Robinson PG, Winkler J, Palmer G et al (1994). The diagnosis of periodontal conditions associated with HIV infection. J Periodontol 65: 236-243.

Rosenstein DI, Eigner TL, Levin MP et al (1989). Rapidly progressive periodontal disease associated with HIV infection: report of a case. J Am Dent Assoc 118: 313-314.

Silness J, Loe H (1964). Periodontal disease in pregnancy. II. Correlation between oral hygiene and periodontal condition. Acta Odontol Scand 22: 121-124.

Swango PA, Kleinman DV, Konzelman JL (1991). HIV and periodontal health. A study of military personnel with HIV. J Am Dent Assoc 122: 49-54.

Todd JE, Lader D (1991). Adult dental health 1988 United Kingdom. OPCS: London, $422 \mathrm{pp}$.

Winkler JR, Herrera C, Westenhouse J et al (1992). Periodontal disease in HIV-infected and seronegative homosexual and bisexual men. AIDS 6: 1041-1043.

Winkler JR, Murray PA, Grassi M et al (1989). Diagnosis and management of HIV-associated periodontal lesions. J Am Dent Assoc 119 (Suppl): 25S-34S.

Winkler JR, Murray PA, Greenspan D et al (1986). Periodontal disease of male homosexuals as related to AIDS virus infection. 2nd International Conference on AIDS, Paris.

Yeung SCH, Stewart GJ, Cooper DA et al (1993). Progression of periodontal disease in HIV seropositive patients. $J$ Periodontol 64: 651-657.

Zambon JJ, Reynolds HS, Genco R (1990). Studies of the subgingival microflora in patients with Acquired Immunodeficiency Syndrome. J Periodontol 61: 699-704. 